DEPARTMENT OF THE INTERIOR

UNITED STATES GEOLOGICAL SURVEY

CHARLES D. WALGOTT, DIRECTOB

\title{
THE ANALYSIS OF SILICATE AND CARBONATE ROCKS
}

BY

W. F. HILLEBRAND 


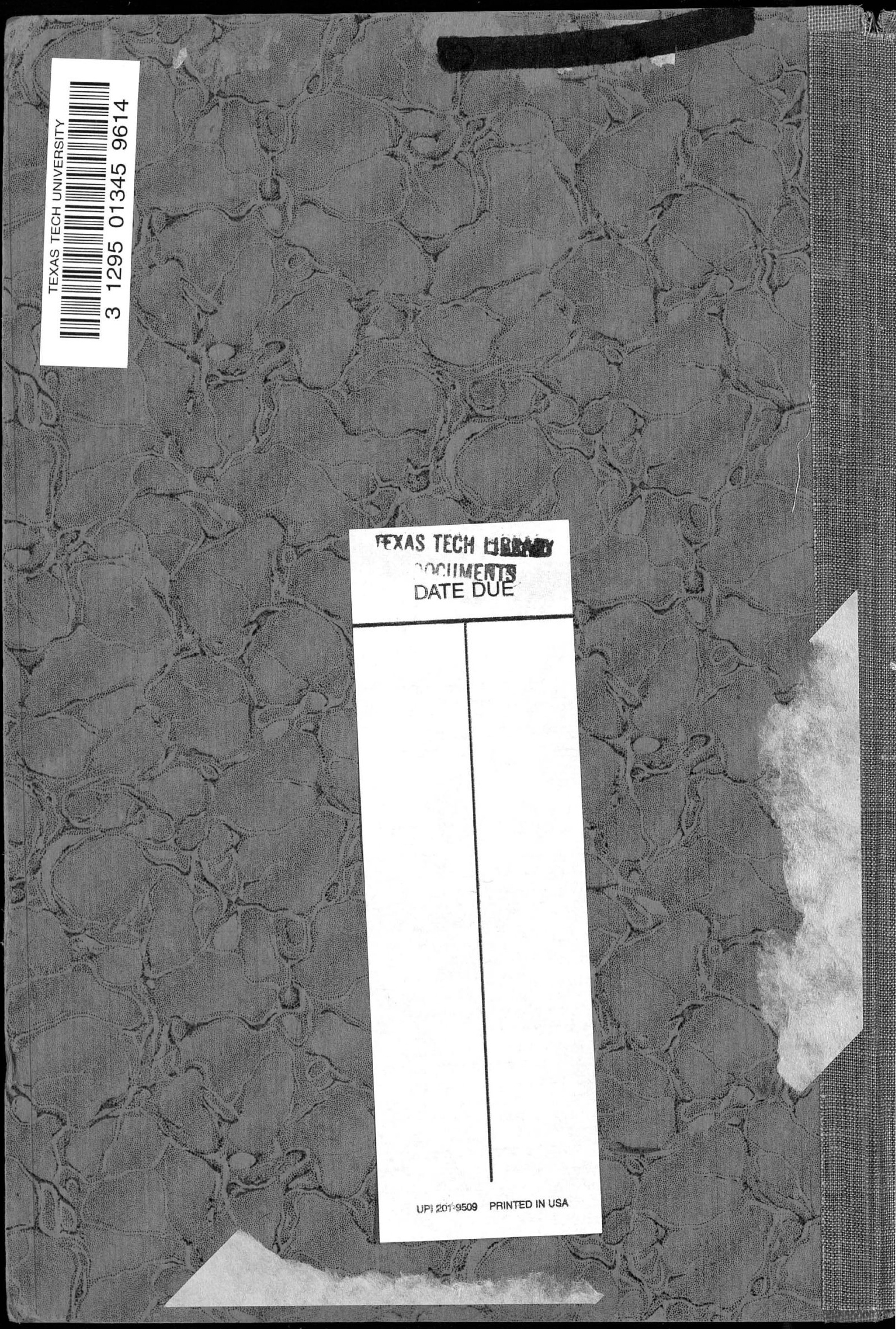


DEPARTMENT OF THE INTERIOR

UNITED STATES GEOLOGICAL SURVEY

CHARLES D. WALCOTT, DIRECTOR

\section{THE ANALYSIS OF \\ SILICATE AND CARBONATE ROCKS}

BY

W. F. HULLEBRAND

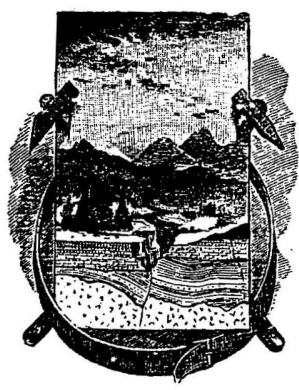

WASHINGTON

GOVERNMENT PRINTING OFFICE

1907 



\section{CONTENTS.}

Page.

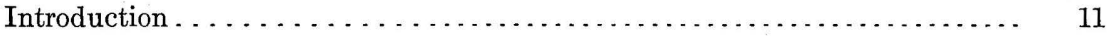

Object and scope of the present treatise . . . . . . . . . . . . . . . 11

Acknowledgments . . . . . . . . . . . . . . . 13

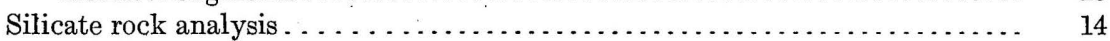

Part I. Introduction . . . . . . . . . . . . . . . . . . . . . . . . . . . . . 14

1. Importance of complete and thorough analyses .............. 14

2. The constituents occurring in silicate rocks . . . . . . . . . . . 20

3. The distribution and occurrence of certain minor constituents .... 21

4. Summation of analytical results and limits of allowable error ..... 25

5. Statement of analyses . . . . . . . . . . . . . . . . . . . . . 27

6. Time needed for making an analysis. ................... 28

7. Useful appliances and apparatus . . . . . . . . . . . . . . 28

A. Crucible tongs . . . . . . . . . . . . . . . . . . . . . 28

B. Radiators for volatilizing liquids and solids ............. 29

C. Perforated disk for crucibles . . . . . . . . . . . . . . . . . . $\quad 30$

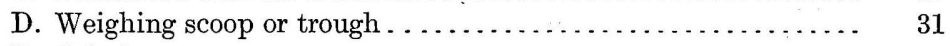

E. Colorimeters . . . . . . . . . . . . . . . . . . . . . . . 31

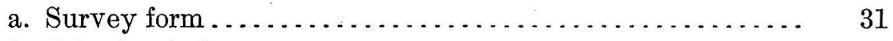

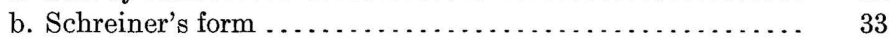

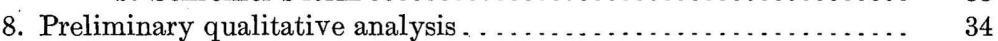

9. Quality of reagents . . . . . . . . . . . . . . . . . . . . . . . . 34

Part II. Methods applicable to silicate rocks . . . . . . . . . . . . . . . . 37

1. Introductory remarks . . . . . . . . . . . . . . . . . . . . $\quad 37$

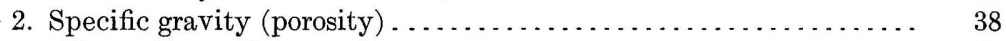

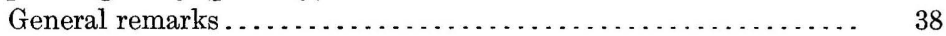

A. By suspension in water . . . . . . . . . . . . . . . . . . . 39

a. Ordinary method for specific gravity . . . . . . . . . . . 39

b. Method for specific gravity and porosity combined . . . . . 40

c. Penfield's method for specific gravity of minerals.... . . . . 42

B. Pycnometer method . . . . . . . . . . . . . . . . . . . . . 442

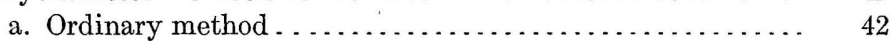

b. Refined method ........................... 43

C. Heavy solutions not suitable for rocks .............. 45

3. Preparation of sample for analysis . . . . . . . . . . . . . 45

A. Quantity of rock to be crushed ................. 45

B. Crushing . . . . . . . . . . . . . 45

a. Survey methods . ......................... 46

b. Washington's method . . . . . . . . . . . . . . . . 47

C. Grinding . . . . . . . . . . . . . . . . . . . . . 47

D. Weight of ground sample...................... 50

4. Water-hygroscopic, zeolitic, crystal . . . . . . . . . . . . . 50

A. General considerations . . . . . . . . . . . . . . . . . . 50

a. Importance of employing air-dry powder for analysis. .... 50

b. Temperature of drying . . . . . . . . . . . . . . . . . 50

c. Indirect methods . . . . . . . . . . . . . . . . . . . . 51

d. Direct method . . . . . . . . . . . . . . . . . . . . 53 
Silicate rock analysis-Continued.

Part II. Methods applicable to silicate rocks-Continued.

4. Water-hygroscopic, zeolitic, crystal-Continued.

A. General considerations-Continued.

e. Argument in favor of, including hygroscopic water in summation . . . . . . . . . . . . . . . . . . . . . . . . . . .

f. Separate entry of hygroscopic and combined water ........

g. Is all true hygroscopic water expelled at $100^{\circ}$ ? . . . . . . . . . . .

B. Apparatus for the direct determination of water at different

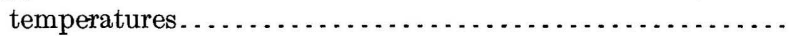

General precautions . . . . . . . . . . . . . . . . . . . . . . . .

a. Steiger's application of the toluene bath ..............

b. Chatard's oven for temperatures up to $300^{\circ}$ and over......

5. Water-total or combined.

A. Arguments against "loss on ignition" method ..............

B. Direct weighing of the water without the use of absorption tubes-Penfield's methods.

a. For minerals easily deprived of their water.............

b. For minerals not easily deprived of their water...........

C. Direct weighing of the water in absorption tubes ............

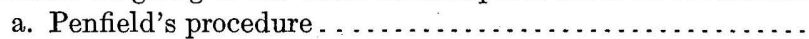

b. Gooch's apparatus . . . . . . . . . . . . . . . . . . . . . . . .

c. Merits of the above forms of apparatus.................

d. Jannasch's methods ............................. $\quad 66$

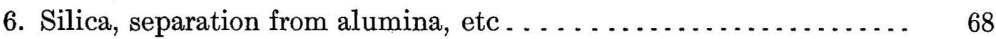

A. Alternative methods of decomposing the rock............ 68

a. General considerations . . . . . . . . . . . . . . . . . . . . . 68

b. Decomposition of refractory silicates by hydrochloric acid under pressure..............................

c. The boric-oxide method of Jannasch and Heidenreich.....

$\alpha$. Preparation of the boric oxide ...................

$\beta$. Treatment of easily decomposable silicates ........

$\gamma$. Treatment of refractory silicates.................

$\delta$. Further treatment after fusion ...................

$\varepsilon$. Possible objections to the boric-oxide method .......

d. The sodium-carbonate method ......................

$\alpha$. Advantages of sodium carbonate over sodium potassium carbonate............................

$\beta$. Purity of the sodium carbonate.................

$\gamma$. The fusion with normal sodium carbonate ...........

$\delta$. Fusion with sodium bicarbonate ..................

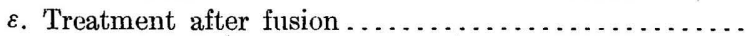

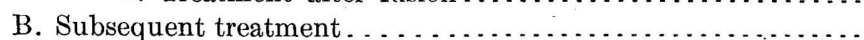

a. Separation of silica . . . . . . . . . . . . . . . . . . . . . . .

$\alpha$. Reasons for adoption of accepted procedure.........

$\beta$. Procedure in absence of notable amounts of fluorine..

$\gamma$. Procedure with rocks and minerals containing fluorine.

b. Ignition of silica

c. Correction for impurities in the silica

d. Accuracy of the silica determination . . . . . . . . . . . . . .

e. Composition of the residue obtained from the silica.......

f. Platinum in filtrates. . . . . . . . . . . . . . . . . . . . . . .

7. Metals precipitable by hydrogen sulphide 
Silicate rock analysis-Continued.

Part II. Methods applicable to silicate rocks-Continued.

Page.

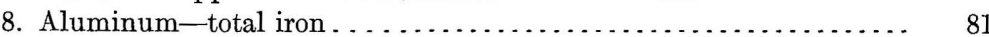

A. Indirect method for aluminum (titanium) . . . . . . . . . . 81

a. Preliminary remarks . . . . . . . . . . . . . . . . . 81

b. Precipitation of aluminum, iron, etc., without precipita-

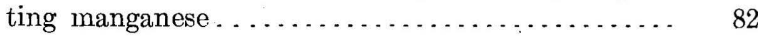

(x. Precipitation by ammonia . . . . . . . . . . . . 82

$\beta$. The basic-acetate precipitation . . . . . . . . . . . 83

c. Precipitation of iron, aluminum, etc., together with manganese . . . . . . . . . . . . . . . . . .

$\alpha$. By ammonia and ammonium persulphate ........

$\beta$. By ammonia and ammonium sulphide. .........

d. Recovery of iron and aluminum from the filtrates.......

$\alpha$. After an ammonia precipitation . . . . . . . . . . . .

$\beta$. After a basic acetate precipitation . . . . . . . . . . . .

$v$. $\Lambda$ fter precipitation by ammonia and ammonium persulphate

e. Ignition of the precipitate of iron, aluminum, etc., oxides

f. Recovery of silica and possible barium in the alumina pre-

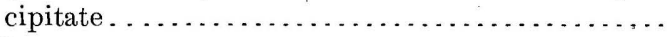

c. Silica . . . . . . . . . . . . . . . . . . . .

$\beta$. Barium . . . . . . . . . . . . . . . . . . . .

B. - Determination of iron in the precipitate of alumina, etc.....

a. Without regard to the presence of vanadium . . . . . . . .

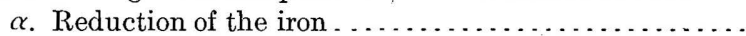

$\beta$. Titration of the iron . . . . . . . . . . . . . . . .

$\gamma$. Further treatment of the solution after titration . . . ....

b. Having regard to the presence of vanadium . . . . . . . . .

c. Determination of the true value for ferric iron..........

C. Methods aiming at the more or less direct determination of aluminum . . . . . . . . . . . . . . . . . . . . . . .

a. After first removing iron as sulphide . . . . . . . . . . . .

b. By extraction with a fixed caustic alkali . . . . . . . . . . .

c. Combination method after Dittrich . . . . . . . . . . . . .

d. Direct precipitation of aluminum by phenylhydrazine...

9. Manganese, nickel, cobalt, copper, zinc . . . . . . . . . . . . .

A. Difficulties in the way of a correct gravimetric determination of manganese . . . . . . . . . . . . . . . . . . . . . . 96

B. Precipitation of the group and separation of its constituents. .

a. The ammonium-sulphide method ...............

$\alpha$. Its advantages and disadvantages . ..............

$\beta$. Precipitation by ammonium sulphide and separation of manganese and zinc from nickel, cobalt, and copper.

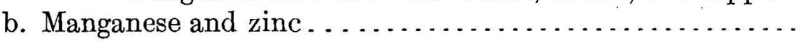

c. Nickel, cobalt, copper . . . . . . . . . . . . . . . . .

C. Colorimetric determination of manganese . . . . . . . . . .

a. Preliminary treatment . . . . . . . . . . . . . . . . . .

b. Colorimetric determination of manganese by ammonium

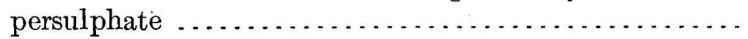

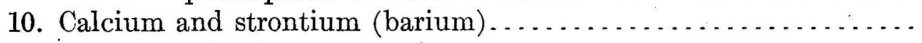

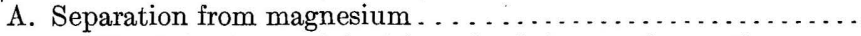

a. Precipitation and ignition of calcium and strontium oxalates together . . . . . . . . . . . . . . . . . . . .

b. Notes on double precipitation, washing, and ignition of the oxalates. 
Silicate rock analysis-Continued.

Part II. Methods applicacle to silicate rocks-Continued.

Page.

10. Calcium and strontium (barium)-Continued.

B. Separation of strontium (barium) from calcium . . . . . . . . 102

a. By ether-alcohol . . . . . . . . . . . . . . . . . . . . 102

b. By amyl alcohol . . . . . . . . . . . . . . . . . . . . . . . 102

C. Behavior of barium . . . . . . . . . . . . . . . . . . . . . 102

D. Separation of barium from strontium. . . . . . . . . . . . 103

E. Other methods of determining the weight of calcium . . . . . . 104

General statement . . . . . . . . . . . . . . . . . . . . . . . 104

a. Volumetric method . ......................... 105

b. By weighing as the carbonate ................... 105

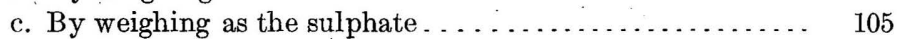

d. By weighing as the fluoride.................... 105

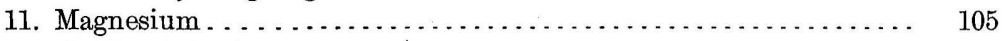

A. Precipitation . . . . . . . . . . . . . . . . . . . . . . . 105

a. Conditions necessary for the production of a normal pre-

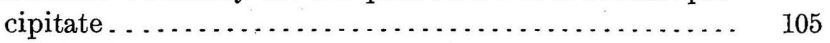

b. Precipitation . . . . . . . . . . . . . . . . . . . . . . 107

$\alpha$. First precipitation ......................... 107

$\beta$. Second precipitation . . . . . . . . . . . . . . . 107

B. Methods of collecting and igniting the precipitate........ 108

a. Ordinary procedures . . . . . . . . . . . . . . . . . 108

b. Modification of Ullbricht. . . . . . . . . . . . . . . 108

C. Contamination by and removal of barium, calcium, and manga-

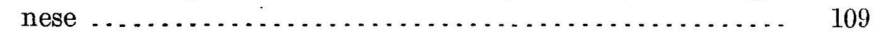

a. Barium .................................... 109

b. Calcium . . . . . . . . . . . . . . . . . . . . . . . 109

c. Manganese .................................. 110

D. Liability to error in the average magnesium determination. . . 109

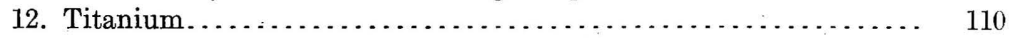

A. Colorimetric determination with hydrogen peroxide. . . . . . 110

a. Description of the method (Weller's) . . . . . . . . . . . 110

b. Correction for the color of ferric sulphate . . . . . . . . . . . 112

c. Alternative mode of preparing the test solution. . . . . . . . 11:;

B. Gravimetric methods . . . . . . . . . . . . . . . . . . . . . . 113

a. Gooch's method . . . . . . . . . . . . . . . . . . . . . 113

$\alpha$. Description of the method................ 113

$\beta$. Gooch's method not directly applicable to rocks con-

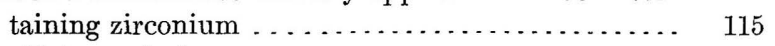

b. Baskerville's method . . . . . . . . . . . . . . . . . . . 115

c. Method of Dittrich and Pohl . . . . . . . . . . . . . . 115

C. Superiority of the colorimetric and Gooch methods over the older ones . . . . . . . . . . . . 116

13. Barium (zirconium, rare earths, total sulphur, chromium) . . . . 116

A. The general method . . . . . . . . . . . . . . . . . . . . 116

B. The method without regard to zirconium and sulphur ...... 118

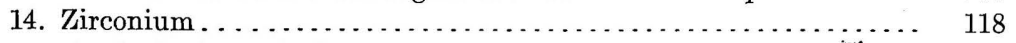

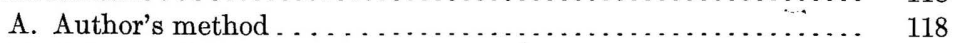

B. Other methods . . . . . . . . . . . . . . . . . . . . . . . 119

15. Rare-earth metals other than zirconium . . . . . . . . . . . . . 120

A. Usual method . . . . . . . . . . . . . . . . . . . . . . . 121

B. Alternative method . . . . . . . . . . . . . . . . . 121 
Silicate rock analysis-Continued.

Part II. Methods applicable to silicate rocks-Continued.

Page.

16. Phosphorus...................................... 121

A. Procedure when material is ample ................... 121

a. Washington's method of preliminary treatment......... 121

b. Alternative method of preliminary treatment............ 122

c. Subsequent treatment........................... 122

B. Procedure when material is scanty .................. 122

17. Chromium..................................... 123

A. Gravimetric method ................................ 124

B. Colorimetric method ............................... 124

$\alpha$. Preparation and strength of standard solution....... 124

$\beta$. Preparation of test solution...................... 124

$\gamma$. Comparison of colors ......................... 124

C. A few comparative data ............................ 125

18. Vanadium (chromium) and molybdenum ................ 126

A. Distribution of vanadium and molybdenum ............. 126

B. Condition of vanadium in rocks..................... 126

C. Author's method ................................. 127

a. Description of the method......................... 127

b. Confirmatory qualitative tests.................... 129

c. Application of the method in presence of relatively much chromium................................. 129

D. Colorimetric method for vanadium.................. 131

19. Ferrous iron ........................................... 131

A. Comparison of sealed-tube and hydrofluoric-acid methodsComparative worthlessness of the former in rock analysis ... 131

B. The modified Mitscherlich method.................... 133

$\alpha$. Strength of acid ........................... 133

$\beta$. Filling, sealing, and heating of the tube ........... 134

$\gamma$. Reason for introducing gas and sealing as above directed .................................. 134

C. The hydrofluoric-acid method . ..................... 135

a. Principle of the method ......................... 135

b. Defects of the method ............................ 135

$\alpha$. Oxidizability of divalent manganese by permanganate in presence of hydrofluoric acid ..............

$\beta$. Extreme oxidizability of divalent iron by free oxygen in presence of hydrofluoric acid or a fluoride.......

c. Influence of sulphides, vanadium, and carbonaceous matter on the determination of ferrous iron by the hydrofluoric-acid method.......................... 138

$\alpha$. Sulphides.................................. 138

ß. Vanadium .................................. 140

$\gamma$. Carbonaceous matter......................... 140

d. The method in its various modifications.............. 140

$\alpha$. According to Cooke ........................... 140

$\beta$. According to Pratt (modified) ................... 142

$\gamma$. According to Treadwell......................... 143

D. Uncertainties of the ferrous-iron determination ............. 144

20. Alkalies......................................... 144

A. The J. Lawrence Smith method....................... 144

a. Its advantages .................................... 144

b. Reagents and apparatus.......................... 144 
Silicate rock analysis-Continued.

Part II. Methods applicable to silicate rocks-Continued.

20. Alkalies-Continued.

A. The J. Lawrence Smith method-Continued.

c. Treatment of the mineral powder................... 145

d. Separation of calcium and sulphuric acid ............. 146

e. Precipitation of potassium.......................... 147

f. Sodium and lithium.......................... 147

$\alpha$. Gooch's method for separating lithium............. 148

B. Separation of alkalies by other methods ............... 148

a. The mercuric-oxide method....................... 149

b. The ammonium-carbonate method .................... 149

c. The amyl-alcohol method ......................... 149

21. Carbon dioxide, carbon............................ 150

A. Qualitative test for carbon dioxide.................. 150

B. Quantitative tests for carbon dioxide.................. 150

a. Direct method ................................ $\quad 150$

b. Indirect method ................................. 152

C. Carbon .......................................... 158

22. Chlorine.......................................... 153

A. Condition in rocks............................. 153

B. Determination of water-soluble chlorine................. 154

C. Determination of acid-soluble chlorine................... 154

a. By nitric acid ................................. 154

b. By nitric and hydrofluoric acids.................. 155

D. Determination of chlorine by alkali fusion.............. 155

23. Fluorine (silica in presence of fluorine) .................... 175

A. Imperfection of the qualitative test for fluorine........... 155

B. Quantitative determination of fluorine .................. 155

a. The method .................................... 155

b. Testing of the calcium fluoride.................... 157

c. Accuracy of the method........................... 158

C. Determination of silica............................. $\quad 159$

24. Sulphur........................................... 159

A. Determination of its condition . . . . . . . . . . . . . . . 159

B. Quantitative determination...................... 160

a. Total sulphur................................. 160

b. Fractional determinations....................... 160

25. Boron ........................................... 161

26. Certain constituents in minute traces ..................... 162

27. The gases and vapors expelled by heat.................. 163

28. Special operations................................... 164

A. Detection of nepheline in presence of olivine ........... 164

B. Determination of soluble silica ....................... 165

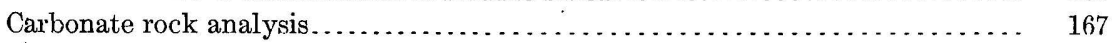

Part I. Introduction ......................................... 167

29. Qualitative comparison of carbonate and silicate rocks ......... 167

30. Mineral composition of carbonate rocks.................... 169

31. Behavior toward different reagents as a means of distinguishing different carbonates ...................................

a. Differing solubility in tartaric and citric acids and in acid potassium sulphate............................

b. The Meigen reaction for distinguishing calcite from aragonite .................................... 172

c. Distinguishing between calcite and dolomite............ 173 
Carbonate rock analysis-Continued.

Part II. Refined methods of analysis . . . . . . . . . . . . . . . . . . . . . 174

32. Silica, its separation from alumina, etc................. . . 174

A. Methods of decomposing the rock.................... . . 174

a. When the inorganic residue is to be separately analyzed... 174

b. When the inorganic residue is not to be separately analyzed 175

$\alpha$. By solution in acid after strong ignition......... 175

$\beta$. By solution in acid after heating with sodium carbonate ............................. 176

B. Separation of silica............................ 176

33. Aluminum, total iron, titanium (silica, manganese); precipitation in company with phosphorus, etc.................. 177

A. Precipitation of aluminum, iron, etc . . . . . . . . . . 178

a. Collective precipitation of aluminum, iron, manganese, titanium, and phosphorus by ammonium sulphide.....

b. Precipitation of aluminum, iron, titanium, and phos-

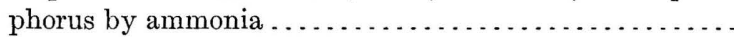

c. Precipitation of aluminum, iron, titanium, and phosphorus by the basic-acetate method...............

B. Treatment of the filtrates from alumina, etc..............

C. Solution and separation of the oxides obtained in A and B...

a. Silica (barium) . . . . . . . . . . . . . . . . . . . .

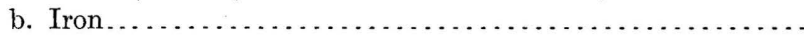

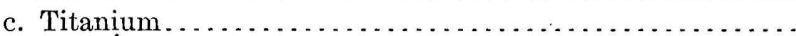

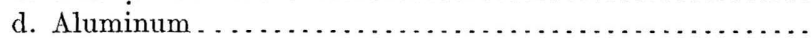

A. In the filtrates from 33 .

. In the filtrates from 33 B........

35. Copper, nickel cobalt, zinc, rare

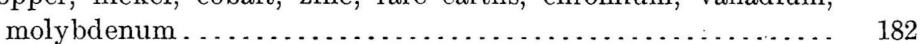

36. Calcium, strontium, barium, magnesium (manganese) ......... 183

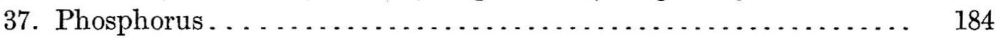

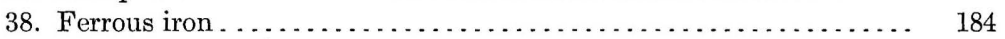

A. In absence of carbonaceous matter . . . . . . . . . . . . . . 184

a. Ferrous iron soluble in sulphuric acid ............. 184

b. Ferrous iron in the insoluble residue . . . . . . . . . . . 185

c. Total ferrous iron . . . . . . . . . . . . . . . . . . . . . . . 185

B. In presence of carbonaceous matter . . . . . . . . . . . . 186

39. Alkalies . . . . . . . . . . . . . . . . . . . . . . . . . . . . . . 186

40. Carbon dioxide, carbon (water) . . . . . . . . . . . . . . . . 186

A. Determination of carbon dioxide................. 186

B. Determination of carbon of carbonaceous matter . . . . . . . . 186

C. Simultaneous determination of water and of total carbon in both forms . . . . . . . . . . . . . . . . . . . . . . . 187

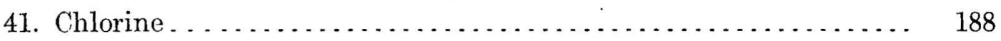

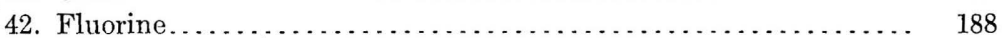

43. Sulphur . . . . . . . . . . . . . . . . . . . . . . . . . . 188

A. Tests as to its condition; determination of sulphate sulphur .. 188

B. Determination of total sulphur. . . . . . . . . . . . . . . . 189

a. Methods of converting sulphides to sulphates . . . . . . 189

$\alpha$. By ignition without flux . . . . . . . . . . . . . . . 189

$\beta$. By ignition with sodium carbonate . . . . . . . . . 189

b. Treatment after ignition . . . . . . . . . . . . . . . . . 189 
Carbonate rock analysis-Continued.

Part II. Refined methods of analysis-Continued.

44. Water ........................................ 190

A. Hygroscopic water........................... 190

B. Combined water............................... 190

Part III. Condensed analysis . . . . . . . . . . . . . . . . . . . . . . . . 190

45. Decomposition and solution . . . . . . . . . . . . . . . . . . . 191

46. Silica.......................................... 191

47. Aluminum, iron, etc . . . . . . . . . . . . . . . . . . . 192

48. Calcium ........................................ 192

49. Magnesium .................................... 193

50. Alkalies . . . . . . . . . . . . . . . . . . . . . . . . . . . . . . 193

51. Carbon dioxide . . . . . . . . . . . . . . . . . . . . . . . . 193

52. Sulphur........................................ 193

53. Water........................................... 193

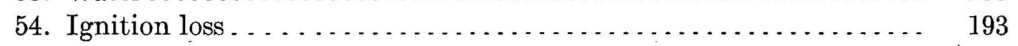

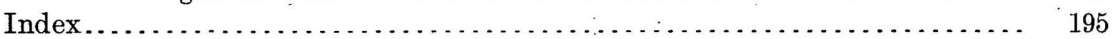

\section{ILLUSTRATIONS.}

Fig. i. Platinum-tipped crucible tongs........................... 28

2. Radiator for rapid and safe evaporation ........................... 29

3. Device for excluding flame gases from crucibles during ignition ...... 30

4. Weighing trough of platinum . . . . . . . . . . . . . . . . . . . . . . 31

5. Survey form of colorimeter . . . . . . . . . . . . . . . . . . . . . 32

6. Schreiner's colorimeter ................................ 33

7. Moore's device to remove air from mineral powders. . . . . . . . . . . 44

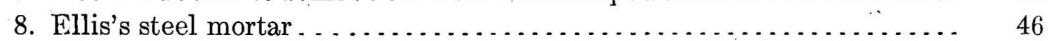

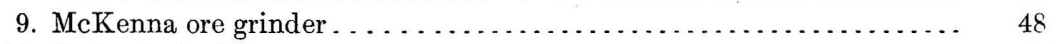

10. Steiger's drying apparatus............................... 57

11. Chatard's drying oven for water determination................. 58

12. Penfield's tubes for water determination....................... $\quad .60$

13. Penfield's oven for water determination . . . . . . . . . . . . . . . . . . 61

14. Penfield tube for water determination........................ 61

15. Modified form of Gooch tubulated platinum crucible for water determination .......................................... 63

16. Arrangement of Gooch apparatus during drying ................. 64

17. Arrangement of Gooch apparatus during fusion..................... 65

18. Glass tube for determination of water (Jannasch) . . . . . . . . . . 66

19. Glass tube for determination of water in special cases (Jannasch) ..... 67

20. Cooke's apparatus for determination of ferrous iron ............... 141

21. Treadwell's apparatus for ferrous-iron determination .............. 143

22. J. Lawrence Smith's crucible for alkali determinations . ............. 145

23. Apparatus for carbon-dioxide determinations . . . . . . . . . . . . 151

24. Kreider's apparatus for indirect determination of volatile substances.. 152 


\title{
THE ANALYSIS OF SILICATE AND CARBONATE ROCKS.
}

\author{
By W. F. Hillebrand.

\section{INTRODUC'TION.}

OBJECT AND SCOPE OF THE PRESENT TREATISE.

The literature relating to analysis of silicates is extensive but scattered, and in no single paper up to the time of the publication of Bulletin No. 148 of this Survey was there to be found in the light of modern methods, a satisfactory exposition of the procedures to be followed or the precautions to be observed, especially in the search for some of the rarer constituents or those which, without being rare, have been of late years recognized as occurring persistently in small amounts. It was not the intention to make the chapter on rock analysis in that bulletin a manual on mineral or even rock analysis, but to show primarily the principles and methods by which the major part of the analyses made up to that time in the laboratory of the Geological Survey had been conducted, and thus to afford a partial measure of the trustworthiness of those analyses. At the same time it was felt that the experience gained by the chemists of the Survey since the establishment of its first chemical laboratory in Denver might be useful to most chemists engaged in mineral and rock analysis. The favorable reception accorded it by chemists led to the republication of a portion of Bulletin No. 148 in more extended form as Bulletin No. 176. Since the publication of the latter bulletin in 1900 two other treatises on rock analysis have appeared: Manual of the Chemical Analysis of Rocks, by Dr. H. S. Washington (1904), and Anleitung zur Gesteinsanalyse, by Dr. Max Dittrich (1905). The former is based largely on Bulletin No. 176; the latter, while adopting some of the methods advocated in that bulletin, adheres more to methods which are in greater favor in Germany than in this country. They both differ from Bulletin No. 176 in devoting less space to a discussion of principles and to the determination of the less common constituents of rocks. Doctor Washington's book enters into considerable detail in describing manipulations, since it was written 
for chemists, petrologists, mining engineers, and others who have not made a particular study of quantitative analysis. These books have not, therefore, rendered the Survey publication superfluous. On the contrary, since some methods have undergone improvement or been superseded by better ones, the time seems ripe for its republication in revised form.

Experience has shown that for the use of the student the outlines of some methods and procedures were too briefly sketched in the earlier publications. Where it seemed called for, therefore, these have received considerable elaboration.

For the reasons that the chief carbonate rocks form so important an element in the composition of the earth's crust, and that the knowledge of their composition is of moment to the geologist as well as to the cement maker, who now makes enormous use of them, a special section devated to their analysis has been added. The methods applied to their analysis differ in but unimportant respects from those used with the more siliceous rocks, since they are to a great extent themselves siliceous, contain essentially the same constituents, and therefore the same principles apply to both. Naturally they demand and receive briefër treatment.

The special problems often arising in the analysis of rocks of extra-terrestrial origin - the more or less stony meteorites-will not be considered here. An analysis of that kind should never be intrusted to the novice, but only to the chemist who has a knowledge of the composition and properties of the peculiar mineral constituents of those bodies and a judgment fit to cope with the oftentimes difficult problems presented by them.

The methods described in the following pages are not restricted altogether to those actually in use in the Survey laboratory. A number of alternative ones are given which are known or believed to be good, in order that those who may wish to use this treatise as a practical guide shall have some choice of selection in case the expensive apparatus or complicated arrangements sometimes preferred are not available. Where silicate and limestone analyses are very frequently made, however, it is a saving of time and money in the end to set up permanent arrangements for convenience in determining water, carbon_dioxide, ferrous iron, in making reductions in hydrogen, etc.

It was my intention to prepare a chapter on the mechanical separation in a small way of minerals by the various processes more or less in vogue, as panning, upward flow of water, heavy solutions, and magnetism, but lack of time, and in fact insufficient experience with some of the methods and solutions, have prevented its development. To those seeking information on some of these methods reference is made to the works of Rosenbusch-Wülfing, Mikroscopische Physiographie der Mineralien und Gesteine, volume 1 (1904); Rosenbusch- 
Iddings, Microscopical Physiography of Rock-making Minerals, fourth edition (1903); and Ferdinand Zirkel, Lehrbuch der Petrographie, second edition, volume 1.

\section{ACKNOWLEDGMENTS.}

To my colleagues, Drs. F. W. Clarke, E. T. Allen, and E. C. Sullivan, and Messrs. George Steiger and W. T. Schaller, my thanks are freely rendered for friendly criticism, suggestions, and occasional direct assistance. 


\section{SILICATE ROCK ANALYSIS. PART I.-INTRODÙCTION.}

1. IMPORTANCE OF COMPLETE AND THOROUGH ANALYSES.

The composition of the ultimate ingredients of the earth's crustthe different mineral species which are there found and of many of which its rocks are made up-was the favorite theme of the great workers in chemistry of the earlier half of the nineteenth century, and for the painstaking care and accuracy of Berzelius, Wöhler, and others the mineralogists and geologists of to-day have reason to be thankful. Considering the limited facilities at their disposal in the way of laboratory equipment and quality of reagents, the general excellence of their work is little short of marvelous. As an outgrowth of and closely associated with the analysis of minerals came that of the more or less complex mixtures of them-the rocks - to aid whose study by the petrographer and geologist a host of chemists have for many decades annually turned out hundreds of analyses of all grades of quality and completeness. With the growth and extraordinary development of the so-called organic chemistry inorganic chemistry gradually fell into a sort of disfavor. In many, even the best, European laboratories the course in mineral analysis, while maintained as a part of the curriculum of study, became but a prelude to the ever expanding study of the carbon compounds, which, multiplying rapidly and offering an easy and convenient field for original research and possible profit, proved more tempting to young chemists than the often worked over and apparently exhausted inorganic field. For one student devoting his time to higher research on inorganic lines fifty perhaps were engaged in erecting the present enormous structure of carbon chemistry. The instruction afforded the student in mineral analysis was confined to the ordinary separations of the commoner ingredients occurring in appreciable quantities, with little regard to supposed traces and with still less attempt to find out if the tabulated list really comprised all that the mineral or rock contained.

With the introduction of improved methods of examination by the petrographer, especially as applied to thin rock sections, and the use 
of heavy solutions, whereby, on the one hand, the qualitative mineral composition of a rock could be preliminarily ascertained with considerable certainty, and on the other chemical examination of the more or less perfectly, separated ingredients was rendered possible, a great help and incentive was afforded to the few chemists engaged in rock analysis. The microscope often obviated in part the necessity for tedious and time-wasting qualitative tests, and the heavy solutions, by permitting the concentration and separation of certain components, facilitated the detection of elements whose existence had long been overlooked.

Meanwhile, in the progress of chemistry new methods and reagents for qualitative detection and quantitative separation and estimation were gradually being devised and discovered. The belief that some well-established methods were adequate was shown to be unwarranted; some had to be discarded altogether; others were still utilizable after modification. In the light thus shed it became possible to explain many hitherto incomprehensible variations in the composition of some rock species or types, as shown in earlier analyses, and in not a few cases it appeared that the failure to report the presence of one or more elements had obscured relations and differences which more thorough examination showed to exist (see p. 17). Consequently there arose a feeling of distrust of much of the older work in the minds of those chemists and petrographers who were best fitted to judge of its probable qualities. This, and the incompleteness of nearly all the earlier work (and much of that of to-day, unfortunately), as shown by the largely increased list of those elements now known to enter into the normal composition of rocks, are rendering the old material less and less available to meet the increasing demands of the petrographer. ${ }^{a}$

And yet these demands on his part are, with few exceptions, by no means so exacting as they should be. Frequently the analysis is intrusted to a student without other experience than that gained by the analysis of two or three artificial salts and as many comparatively simple natural minerals, and with a laboratory instructor as adviser whose experience in rock analysis may be little superior to his own. In other words, one of the most difficult tasks in practical analysis is expected to be solved by a tyro, and his results are complacently accepted and published broadcast without question. Even to those thoroughly familiar with the subject rock analysis is a complex and often trying problem. Although long practice may have enabled

$a$ Dr. Henry S. Washington, in Professional Papers Nos. 14 (Chemical analyses of igneous rocks published from 1884 to 1900 , with a critical discussion of the character and use of analyses) and 28 (The superior analyses of igneous rocks from Roth's Tabellen, 1869 to 1884, arranged according to the quantitative system of classification) of the United States Genlogical Surver, has done a most important work in sifting the great mass of data accumulated in the thousauds of analyses published since 1869. After a systematic critical examination he has assigned to each analysis a certain value on a scale of 5 . Many of these values may be incorrect, but in most cases they undoubtedly give a fairly true measure of the weight to be attached to a particular analysis, 
one to do certain parts of it almost mechanically, perplexing questions still arise which require trained judgment to properly meet and answer, and there is yet room for important work in some of the supposedly simplest quantitative.determinations. If the results are to have any decided value for purposes of scientific interpretation and comparison, they must be the outcome of the work of one who is able to find his way through the intricacies of an analysis in which from fifteen to twenty-five components are to be separated and estimated with close approach to accuracy, and this a beginner can not hope to do. The conscientious chemist should have a live interest in this matter. $\mathrm{He}$ should work with a twofold purpose in view-that of lightening the labors of those who come after him by enabling them to use his work with less supplementary examination, and that of enhancing his own reputation by meriting encomiums on work that has stood the test of time.

How little understood may be the principles underlying the treatment of bodies so complex and the accurate separation and determination of their constituents, even when these are comparatively few in number, has been strikingly shown during the last five years in the work of several committees of chemists charged with the investigation of the methods employed in various lines of technical chemistry involving the analysis or assay of zinc ores, slags from the smelting of copper ores, argillaceous limestones, and cements. In all cases a most woful inability to obtain agreeing results is apparent, not only among those less experienced, but among those supposed to be most expert in each of the particular fields as well. Some improvement in silicate analysis has resulted from the investigations set on. foot and the recommendations made by these committees, and further improvement may be expected, but the situation is yet anything but satisfactory. The art of analysis stands in great need of more thorough treatment in our educational institutions.

The petrographer, again, should seek to have his analyses made as complete as possible, and not. as is so often the case, be content with determinations of silica, alumina, the oxides of iron, lime, magnesia, the alkalies, and water. The latter course, it is true, is entirely justifiable at times, and may serve the immediate purposes of the analyses, but their incompleteness may, on the other hand, not only conceal points fruitful of suggestion to the attentive mind, but, what is of still greater importance, they may.be actually misleading. Enough instances of totally inaccurate conclusions to be drawn from them have fallen under my own observation to fully justify this plea in favor of greater completeness in rock and mineral analysis made for purely scientific purposes. $^{a}$

a For a further presentation of this subiect from the view point of an experienced petrographer, see Washington, H. S., Manual of the Chemical Analysis of Rocks, 1904, pp. 8-17. 
The importance of the points indicated in the foregoing paragraph is shown by the difference between the analyses given below. The specimens were taken and analyzed at widely separated times and by different persons, it is true, but they were unquestionably from the same rock mass, in which, however much the relative proportions of the different mineral constituents might vary within certain limits, there can be no reason to doubt the general distribution of all the elements shown by the second analysis.

Analyses of specimens taken from the same rock mass at different dates.

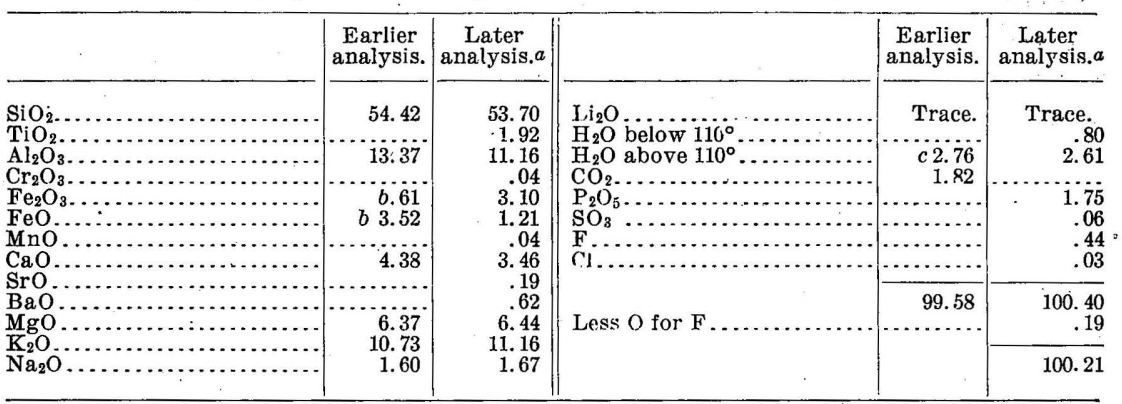

a A still more recent analysis of another of the series of rocks of which this is an example has shown that this "later analysis" is itself probabiy incomplete and incorrect in part-incomplete because of the probable presence of 0.2 per cent or more of $\mathrm{ZrO}_{2}$, incorrect because of the error in $\mathrm{Al}_{2} \mathrm{O}_{3}$ resulting from having counted the $\mathrm{ZrO}_{2}$ as $\mathrm{Al}_{2} \mathrm{O}_{3}$, and from the fact. that titanium is not fully precipitable in presence of zirconium by Gooch's method (the one employed). This latter error involves both the TiOs and the $\mathrm{Al}_{2} \mathrm{O}_{3}$. (See $\beta$, p. 115.)

$b$ From the fact that repeated determinations of the iron oxides in this and related rocks from the same region show always a great preponderance of ferric oxide, it is not improbable that the. figures given for the two oxides in the first analysis were accidentally transposed.

gin the published analvsis it does not appear whether this is total water or, as seems probable, only that remaining above $100^{\circ}$.

Another instance of similar kind is given below. Here, again, certain differences are explainable by natural variations in the proportions of the constituent minerals, but it can hardly be doubted that $\mathrm{TiO}_{2}, \mathrm{BaO}, \mathrm{SrO}, \mathrm{P}_{2} \mathrm{O}_{5}$, and $\mathrm{SO}_{3}$ were present in both specimens in approximately the same amounts. In the earlier analysis determinations of some supposedly unimportant constituents were purposely omitted, or made only qualitatively, with results that can not be otherwise than fatal to a full comprehension of the mineralogical nature of the rock.

Analyses of specimens taken from the same rock mass at different dates.

\begin{tabular}{|c|c|c|c|c|c|}
\hline . & $\begin{array}{l}\text { Earlier } \\
\text { analysis. }\end{array}$ & $\begin{array}{c}\text { Later } \\
\text { analysis. }\end{array}$ & & $\begin{array}{c}\text { Earlier } \\
\text { analysis. }\end{array}$ & $\begin{array}{c}\text { Later } \\
\text { analysis. }\end{array}$ \\
\hline $\mathrm{SiO}_{2}$. & 44. 31 & 44.65 & \multirow{9}{*}{ 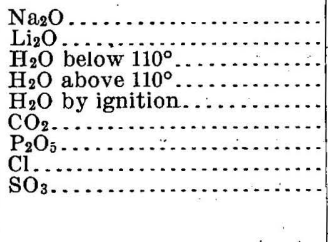 } & 4.45 & 5.67 \\
\hline $\mathrm{TiO}_{2}$. & Not est. & .95 & & & Trace. \\
\hline $\mathrm{Al}_{2} \mathrm{O}_{3}$ & 17. 20 & 13.87 & & .77 & .95 \\
\hline $\mathrm{Fe}_{2} \mathrm{O}_{3}$ & 4.64 & 6.06 & & & 2.10 \\
\hline $\mathrm{FeO}$ & 3.73 & $\begin{array}{r}2.94 \\
.17\end{array}$ & & 3.30 & .ii \\
\hline $\begin{array}{l}\mathrm{MnO} \ldots \ldots \ldots \ldots \ldots \\
\mathrm{CaO} \ldots \ldots \ldots \ldots\end{array}$ & 10.40 & $\begin{array}{r}.17 \\
9.57\end{array}$ & & & 1.50 \\
\hline SrO. & $\therefore \ldots \ldots \ldots$ & a. 37 & & & Trace. \\
\hline $\mathrm{BaO}$. & \multirow{2}{*}{$\begin{array}{l}6.57 \\
3.64\end{array}$} & $\begin{array}{r}.76 \\
5.15\end{array}$ & & & \\
\hline$\overline{\mathbf{K}}_{2}^{c}$ & & 4. 49 & & 99.11 & 99.92 \\
\hline
\end{tabular}

$a$ Not entirely free from $\mathrm{CaO}$,

Bull. 305-07-2 
Prof. F. W. Clarke has shown that the combined percentages of titanic and phosphoric oxides in rocks of the earth's crust, averaged from hundreds of analyses, amount to 0.8 per cent. When the determination of these is neglected the error falls on the alumina.. If the alumina is then used as a basis for calculating the feldspars, it is easy to see that a very large average error in the latter may result, amounting to several per cent of the rock.

In order to emphasize more strongly the importance of completeness in analysis, a few facts brought out by the hundreds of rock analyses made in this laboratory may be cited. It has been demonstrated most conclusively that barium and strontium are almost never-failing constituents of the igneous rocks of the United States and of many of their derivatives. The amounts are usually below 0.1 per cent for each of the oxides of those metals, but higher amounts are by no means uncommon. Furthermore, the weight of barium is almost without exception in excess of that of strontium; but a still more important point is that the igneous rocks of the Rocky Mountain region, so far as examined, show far higher average percentages of both metals than the rocks from the eastern and the more western portions of the United States. The following examples serve to illustrate certain types of Rocky Mountain igneous rocks: Of seven rocks forming a Colorado series, six held from 0.13 to 0.18 per cent of $\mathrm{BaO}$, while in the seventh the percentage was 0.43 . The SrO ranged from 0.07 to 0.13 per cent for six and was 0.28 for that one highest in $\mathrm{BaO}$. Of thirteen geologically related rocks from Montana, embracing basic as well as acidic and intermediate types, the range of $\mathrm{BaO}$ was from 0.19 to 0.37 per cent, with an average of 0.30 per cent. Three others of the same series contained 0.10 per cent or less, while the seventeenth carried 0.76 per cent $\mathrm{BaO}$. The $\mathrm{SrO}$ ranged from 0.37 per cent in the last instance to an average of 0.06 for the other sixteen. Certain peculiar rocks from Wyoming carry from 0.62 to 1.25 per cent $\mathrm{BaO}$ and from 0.02 to 0.33 per cent SrO. Surely this concentration of certain chemical elements in certain geographic zones has a significance which future geologists will be able to interpret if those of to-day are not:

Again, vanadium is an element which few chemists have ever thought of looking for in igneous rocks, though it has long been known to occur in magnetites and other iron ores. A. A. Hayes, in 1875 , reported its occurrence in a great variety of rocks and ores. To quote from Thorpe's Dictionary of Chemistry: "It is said to be diffused with titanium through all primitive granite rocks (Dieulafait), and has been found by Deville in bauxite, rutile and many other minerals and by Bechi and others in the ashes of plants and in argillaceous limestones, schists, and sands." It is further reported to 
comprise, as the pentoxide, up to 0.1 per cent of many French and Australian $^{a}$ clays, 0.02 to 0.03 per cent of some basalts, 0.24 per cent of a coal of unknown origin, and 0.45 per cent of a coal from Peru. Still later examinations in this laboratory of about 100 rocks, chiefly igneoùs, covering nearly the whole continental territory of the United States, show not only its general qualitative and quantitative distribution, but that it predominates in the less siliceous igneous rocks and is absent, or nearly so, in those high in silica. In some of the more basic rocks it occurs in sufficient amount to affect seriously the figures for the oxides of iron unless separately estimated and allowed for (see $\beta$, p. 140), a matter of considerable importance, since the petrographer lays great stress on accuracy in their determinations.

The same investigation has also thrown some light on the distribution of molybdenum, which seems to be confined to the more siliceous rocks and to occur in quantities far below those commonly found for vanadium.

Finally, had it not been my practice of late years to look for sulphur in rocks, even when no sulphides were visible to the eye, its almost invariable presence in the form of sulphide and consequent connection with the long mystifying lack of agreement between results for ferrous iron obtained by the Mitscherlich and the hydrofluoric-acid methods might not have been suspected (see A, p. 131).

While strongly upholding the necessity for more thorough work, necessarily somewhat at the expense of quantity, it is far from my intention to demand that an amount of time altogether disproportionate to the immediate objects to be sought should be expended on every analysis. But it is maintained that, in general, the constituents which are likely to be present in sufficient amount to admit of determination in the weight of a sample usually taken for analysis-say 1 gram for $\mathrm{SiO}_{2}$, $\mathrm{Al}_{2} \mathrm{O}_{3}$, etc., to 2 grams for certain other constituents - should be sought for, qualitatively at least, in the ordinary course of quantitative work, and their presence or absence noted among the results. If present in little more than traces, that knowledge alone may suffice, for it is often more important to know whether or not an element is present than to be able to say that it is there in amount of exactly 0.02 or 0.06 per cent. In the tabulation of analyses a special note should be made in case of intentional or accidental neglect to look for substances which it is known are likely to be present. Failure to do this may subject the analyst to unfavorable criticism when, at some future time, his work is reviewed and the omissions are discovered by new analyses.

Finally, whenever possible, a thorough microscopical examination of the rock in thin section should precede the chemical analysis. This

a J. C. H. Mingaye has lately confirmed its wide distribution in Australian rocks, coals, etc. (Records Geol. Survey New South Wales, vol. 7, pt. 3, 1903, p. 213.) 
may be of the greatest aid to the chemist in indicating the presence of unusual constituents or of more than customary amounts of certain constituents, whereby, possibly, necessary modifications in the analytical procedure may be employed without waste of time or labor. ${ }^{a}$

If the point be raised that many of the published analyses emanating from the Survey laboratories, even my own earlier ones, are not in accord with the advocacy of completeness contained in the foregoing pages, it may be remarked that these ideas have been to a considerable degree evolved during a personal experience of over twentyfive years in this line of work, and that frequently the exigencies were such as to compel restriction in the examination. Where the latter has been the case, subsequent developments have in some cases shown it to be bad policy in every respect. It is better, for both the geologist and the chemist, to turn out a limited amount of thorough work than a great deal of what may ultimately prove to be of more than doubtful utility.

\section{THE CONSTITUENTS OCCURRING IN SILICATE ROCKS.}

It is to the decomposition of the original igneous rocks or their magmas and their derivatives that nearly all ore bodies in the United States owe their origin by one ór another process of concentration. A certain class of concentrations probably separated from magmas in the fluid state before solidification. Hence it is the natural and inevitable inference that sufficiently careful examination of these rocks would show them to contain all or nearly all the known elements, not necessarily all in a given rock, but many more than anyone has yet found. ${ }^{b}$ Mechanical and analytical difficulties have thus far stood in the way of experimental proof that this is so, owing to the great scarcity of -many of the elements. In exceptional cases it may be desirable to subject a given material to very exhaustive analysis, as, for instance, when searching for the origin of ore deposits. Ordinarily, however, the demands of the petrographer and geologist are satisfied with a knowledge of the quantitative relations of those constituents which can be determined in a limited amount of the sample - say from one-half gram to 2 , or occasionally 5 , grams. In general the discussion relating to silicate analysis in this volume will be confined to such separations as may be required in the analysis of an igneous, metamorphic, or sedi-

\footnotetext{
$a$ See also Washington, H. S., Chemical Analysis of Rocks, 1904, pp. 6-7. The foregoing tables and accompanying remarks, including several sentences preceding the tables, have been largely taken from my paper entitled A plea for greater completeness in chemical rock analysis: Jour. Am. Chem. Soc., vol. 16, 1894, pp. 90-93; Chem. News, vol. 69, 1894, p. 163 . See also Distribution and quantitative occurrence of vanadium and molybdenum in rocks of the United States: Am. Jour. Sci., 4th ser., vol. 6, 1898, p. 209; Chem. News, vol. 78, 1898, p. 216: and Bull. U. S. Geol. Survey No. 167, 1900, p. 49.

$b$ F. Sand berger's researches have shown to what extent this is true of a large number of those elements contributing to the filling of metalliferous veins, and L. Dieulafait by his elaborate qualitatıve researches showed how universal was the distribution of copper, zinc, barium, strontium, etc., in the primordial rocks.
} 
mentary rock of complex mineralogical composition, in which the majority and possibly all of the ingredients in the list given below may occur in weighable or readily discoverable quantities:

$\mathrm{SiO}_{2}, \mathrm{TiO}_{2}, \mathrm{ZrO}_{2}, \mathrm{Al}_{2} \mathrm{O}_{3}, \mathrm{Fe}_{2} \mathrm{O}_{3}, \mathrm{Cr}_{2} \mathrm{O}_{3}, \mathrm{~V}_{2} \mathrm{O}_{3}, \mathrm{FeO}, \mathrm{MnO}, \mathrm{NiO}, \mathrm{CoO}$, $\mathrm{MgO}, \mathrm{CaO}, \mathrm{SrO}, \mathrm{BaO}, \mathrm{ZnO}, \mathrm{CuO}, \mathrm{K}_{2} \mathrm{O}, \mathrm{Na}_{2} \mathrm{O}, \mathrm{Li}_{2} \mathrm{O}, \mathrm{H}_{2} \mathrm{O}, \mathrm{P}_{2} \mathrm{O}_{5}, \mathrm{~S},{ }^{a}$ $\mathrm{SO}_{3}, \mathrm{C},{ }^{b} \mathrm{CO}_{2}, \mathrm{Fl}, \mathrm{Cl}, \mathrm{N}$.

To the above list might be added certain others, as the group of so-called rare earths, besides tin, platinum, tantalum, columbium, boron, glucinum, helium. Some of these occur at times in determinable amounts, though in my experience tantalum, columbium, and glucinum have not been met with. They may very well have been overlooked by reason of the absence of distinctive tests for identification. Thorium, cerium, and other rare earths are probably more common as constituents of silicate rocks than has been generally supposed. Their presence and amount can be so readily and certainly detected by the methods given in their proper place that the reason for neglecting to look for them is no longer so strong as it used to be, especially when there is microscopic or other evidence of the presence of minerals likely to contain them.

\section{THE DISTRIBUTION AND OCCURRENCE OF CERTAIN MINOR CONSTITUENTS.}

For the suggestion of this section and for much of its contents I am in no small degree indebted to Doctor Washington's book (Manual of the Chemical Analysis of Rocks, pp. 18-21), already more than once referred to.

Doctor Washington says:

"The increased number of analyses of igneous rocks, especially of unusual types, and the more frequent determination of the minor constituents, with the vast mass of data obtained by the use of the microscope, have shown that certain of the rare elements are prone to occur in rocks of certain chemical characters. While our knowledge along this line is far from complete, a few words may be devoted to this subject, as it will often be of use to the analyst to know which elements should be especially looked for and which may be safely neglected."

Titanium.-Not long ago held to be rare, this element is now recognized to be one of the most universally distributed and about tenth in actual abundance in the explored crust of the earth. So far as my experience goes, it is entirely absent from no igneous, metamorphic, or sedimentary rock of a more or less siliceous character. The recognition of this fact has been rendered possible and easy by its characteristic behavior, when in solution, toward hydrogen peroxide. Though

a Usually as pyrite, occasionally as lazurite, not infrequently as pyrrhotite.

$b$ As graphite or coaly matter. 
seemingly present even in the most siliceous rocks, it is most abundant in the so-called basic ones. Its chief mineral occurrences are as rutile, octahedrite or anatase, ilmenite, titanite, and perofskite, but it is also a component in smaller amounts of many pyroxenes, hornblendes, biotites, and garnets. Owing to the refractory nature of some of its compounds it tends to concentrate in the residual products of decomposition of many rocks; hence its high percentage in clays as a class. It is very unusual to find titanium present in amounts that can properly be designated only as traces. In the great majority of cases its amount will not exceed 1 per cent, but it may rise to over 5 per cent.

Zirconium.-The chemically related element zirconium is likewise now known to be more widely distributed than was formerly supposed, but it is much less common than titanium, rarely making up 0.2 per cent of a rock and being usually under 0.05 per cent. " It is most apt to occur in granites, rhyolites, syenites, and in nephelite syenites, phonolites, tinguaites, and tephrites, and is most abundant in those which are high in soda, such as the last four. It is rarely met with in the more basic rocks, especially those rich in lime, magnesia, and iron. Zirconium is usually found as the silicate zircon, especially in granites and syenites, but is also an ingredient of the rare minerals eudialyte, låvenite, and rosenbuschite."

Chromium.- "Chromium is almost wholly confined to the basic rocks, especially those which are high in magnesia and low in silica, and consequently contain abundant olivine, such as peridotite and dunite. It occurs as chromite and picotite (chrome-spinel), and in some augites, biotites, and olivines. It may occur up to one-half of 1 per cent of $\mathrm{Cr}_{2} \mathrm{O}_{3}$."

Vanadium.-The distribution of this element has been in part covered by the remarks on pages 18-19. To leave out of account the wellcharacterized mineral vanadates, its most stable and normal form in the silicates seems to be that of trivalency, corresponding to the oxide $\mathrm{V}_{2} \mathrm{O}_{3}$, in which form it replaces alumina and possibly ferric oxide to a very limited extent-a few hundredths of one per cent-in pyroxenes, hornblendes, and biotites. It hence predominates in the less siliceous igneous rocks. As the mineral roscoelite, essentially a vanadioalumino-potassic silicate, or some other similar silicate, it is rather widely distributed as a component of certain sandstones in western Colorado and eastern Utah, sometimes in large amount. ${ }^{a}$ It may well exist in a similar condition in clays and the ashes of coals. The conclusion being based on the above observations, it should not occur as a constituent of nonaluminous and nonferric rock-forming minerals.

a Hillebrand, W. F., and Ransome, F. L., Am. Jour. Sci., 4th ser., vol. 10, 1900, p. 120; Bull. U. S. Geol. Survey No. 262, 1905, p. 9. 
As to olivine, at least, this conclusion has been found to be justified. Vanadium is also a constituent of ilmenite in titaniferous iron ores. The existence of the copper sulphovanadate, sulvanite, and the occurrence of vanadium in apparent combination with sulphur in certain peculiar carbonaceous ores of eastern Utah and Peru ${ }^{a}$ indicate a possible wider range of combination in exceptional cases.

Manganese.-This element is found in ferromagnesian minerals in nearly all rocks, though as the result of their alteration it may sometimes appear, particularly on surfaces of limestones and sandstones, in a more or less peroxidized condition. Its amount will rarely exceed 0.3 per cent. I fully agree with Doctor Washington in regarding the high figures commonly reported as due to analytical error. There is no element which the average chemist is more prone to report too high, by gravimetric methods.

Nickel and cobalt.-These elements are found in the olivine of peridotite rocks and also in pyrite and pyrrhotite, hornblende, and biotite. Even in peridotites the percentage of nickel will seldom exceed 0.1 per cent, while that of cobalt rarely if ever exceeds a trace. As with manganese, the percentage of nickel has often been reported too high.

Copper.-Owing to the extreme precautions necessary to exclude its introduction from utensils and reagents during analysis, there is less positive information regarding the distribution of copper than of some other elements which do not exceed it in amount. Nevertheless, there is reason to believe that it is almost if not quite as universally distributed as most of the other minor constituents. Its particular home appears to be in diabase, gabbro, amphibolite, and other basic rocks with pyroxene and amphibole. J. B. Harrison, in a recent report (1906) to the Science and Agriculture Department of British Guiana, shows its occurrence in hundredths of 1 per cent in many igneous rocks of that colony. So far as my experience goes, it can be found almost invariably, if looked for, in the rock analyses carried out in the Survey laboratory. But for the reason above given it is seldom reported unless extra precautions have been taken to prevent its extraneous entry into the analysis.

Barium and strontium.-Feldspathic rocks are those most likely to carry these elements, the former almost always in excess of the latter. According to Washington, there is considerable evidence, in part unpublished, that barium is apt to be most abundant in rocks which are high in potassium. It occurs "in orthoclase (as the hyalophane molecule) and possibly also in labradorite and anorthite (as celsian) as well as in a few biotites and muscovites." It has been found in a few peculiar rocks in excess of 1 per cent, in terms of the oxide, but in the greater part of the silicate rocks of the United States its percent-

$a$ As to the Peruvian occurrence see Hewett, Foster, Eng. Min. Jour., vol. 82, 1906, p. 385, and Bravo, José J., Bol. Soc. Ing. (Lima), vol. 8, 1906, p. 171, 
age is well below 0.2 . Strontium has been found as high as 0.3 to 0.4 per cent, but generally there is little more than a trace of it.

Lithium.-Although one of the most universally distributed of the elements, it is almost never met with in rocks in more than spectroscopic traces. Aside from the lithium minerals lepidolite and spodumene, it is found in the alkali feldspars, in muscovite, beryl, and other minerals. According to Washington, there is reason for the belief that it is especially prone to occur in highly sodic rocks.

Phosphorus.-This element is found in greatest abundance in the more basic igneous and metamorphic rocks, and is practically never entirely absent. It especially affects those rocks "which are high in lime and iron rather than in magnesia." Its chief mineral occurrence is in apatite, though it may be found in xenotime and monazite. While the percentage is usually well under 1 , it may considerably exceed this figure.

Sulphur.-Aqueous extraction of a powdered rock will in manycases remove traces of sulphur and chlorine. These are to be regarded in almost every case rather as derived from infiltrating waters than as products of decomposition of the constituents of the rock itself. Essential rock-forming minerals containing sulphur in the oxidized condition are limited to hauynite and noselite, minerals which are found chiefly in the more basic rocks, and especially those high in soda. In the sulphide condition the element is of very wide distribution, both as pyrite and pyrrhotite, less often as chalcopyrite and possibly other sulphides, also in the mineral lazurite, and here too in greater abundance in the basic rocks. It is a very common error of most chemists to report the sulphur in silicate and carbonate rocks, clays, etc., as $\mathrm{SO}_{3}$ instead of $\mathrm{S}$. It may now and then occur in both states, but much more often only in the sulphide condition.

Chlorine.-"Chlorine is present most abundantly in rocks which are high in soda, and especially when so low in silica that nephelite is present, though it is also found sometimes in nephelite-free rocks, and in a few cases in quartz-bearing ones. It is an essential component of sodalite [also noselite--W. F. H.], and is also present in scapolite and in a few apatites." Its amount when present rarely exceeds $0: 2$ or 0.3 per cent. When seeking for the chlorine of these minerals it is always well to extract the powder first with cold water (see preceding paragraph). It is to be remembered that fluid inclusions in minerals sometimes contain sodium chloride, which would be largely extracted from the powder by water.

Fluorine.-"Fluorine seems to have no special preference as to magma, though, on the whole, it is found more frequently in acid than in basic rocks. It is also apparently most apt to be met with as fluorite in rocks containing nephelite, as foyaites and tinguaites. It is an essential constituent of fluorite and most apatite, and as an integral 
part of the last mineral is almost universally present. It also occurs in biotites and other micas, in some hornblende and augite, as well as in tourmaline, topaz, chondrodite, etc." When determined it is usually reported in amounts under 0.1 per cent, but freedom of the weighed calcium fluoride from contamination being assumed, the reported amount is invariably too low because of the inaccuracy of the method for its separation (see c, p. 158).

Other minor constituents.- "Glucinum, as a component of beryl, is most frequent in granites, pegmatites, and quartzose gneisses. Tin is confined to the acid rocks, granite, quartz porphyry, and rhyolite, and its presence is due [often-W. F. H.] to pneumatolytic processes. It occurs as cassiterite, and in traces in ilmenite, micas, and feldspars. The rare-earth metals occur in allanite, xenotime, monazite, and other minerals of even greater rarity, and seem to be especially frequent in acid rocks and possibly those with much soda. Molybdenum tungsten, and uranium are almost exclusively confined to the very siliceous rocks: Zinc has been met with in granite, as well as in basic rocks, but no generalization in regard to it is possible as yet [nor in regard to lead, which can often be found by using considerable amounts of rock material, as in some of the rocks of Leadville, Colo., and in those of British Guiana.-W. F. H.] Platinum is found almost exclusively in peridotites, but is occasionally met with in connection with gabbros. Boron, as a constituent of tourmaline, is most apt to occur in highly siliceous rocks." Boron is also common in many contact-metamorphic schists. Gold and silver have been found repeatedly. According to Harrison, ${ }^{a}$ some of the British Guiana rocks contain gold enough to account for the commercially valuable deposits in residual soils resulting from the rock decomposition. Tantalum and columbium occur in some granites and pegmatites, but have never been reported in percentages in rock analysis, so far as known to me.

\section{SUMMATION OF ANALYTICAL RESULTS AND LIMITS OF ALLOWABLE ERROR.}

As is well known, a complete silicate-rock analysis which foots up less than 100 per cent is generally less satisfactory than one which shows a summation somewhat in excess of 100 . This is due to several causes. Nearly all reagents, however carefully purified, still contain, or-extract from the vessels used, traces of impurities, which are eventually weighed in part with the constituents of the rock. The dust entering an analysis from first to last is considerable, washings of precipitates may be incomplete, and if large filters are used for small precipitates the former may easily be insufficiently washed. 
Given the purest obtainable reagents, an ample supply of platinum, facilities for working, and a reasonably clean laboratory, there is usually little excuse for failure on the part of a competent chemist to reach a summation within the limits 99.75 and 100.50. Failure to attain 100 per cent in several of a series of analyses of similar nature should be the strongest evidence that something has been overlooked. Excess above 100.5 per cent should be good ground for repeating portions of the analysis in order to ascertain where the error lies, for it is not proper to assume that the excess is distributed over all determined constituents. It is quite as likely, in fact more than likely, to affect a single determination and one which may be of importance in a critical study of the rock from the petrographic side.

Doctor Washington would extend the allowable limits above given to 99.50 and 100.75. If the analysis has to be made in other vessels than those of platinum a higher limit than 100.50 is certainly called for, but it is extremely doubtful if a lower one than 99.75 should be accepted, for, as has been said, there is a tendency toward too high results in any case, and a summation of less than 99.75 with vessels other than platinum would be indicative of rather gross error on the part of the analyst.

As to the allowable variation in duplicate determinations of the same constituent no rigid rules can be laid down. Doctor Washington has proposed the following, ${ }^{a}$ with which the independent experimental values of Doctor Dittrich ${ }^{b}$ are in substantial agreement: For $\mathrm{SiO}_{2}$ and others which amount to 30 per cent and over, from 0.2 to 0.3 per cent; for $\mathrm{Al}_{2} \mathrm{O}_{3}$ and others which amount to from 10 to 30 per cent, 0.1 to 0.2 per cent; for constituents which amount to from 1 to 10 per cent, 0.05 to 0.1 per cent. These percentages are in terms of the whole rock and not of the particular constituent.

While it is desirable sometimes, and for the beginner imperative; to make duplicate determinations, agreement is not to be taken as proof that the results are correct unless they have been arrived at by different methods.

Too great stress can not be laid upon the importance, especially for the analyst of limited experience, of consistently testing most of his final precipitates and filtrates as carefully as possible in order to be sure, on the one hand, that the substances reported by a given name are wholly such and, on the other, that all is reported. In no other way can an analyst so soon acquire the needed confidence in himself and his methods.

a Manual of the Chemical Analysis of Rocks, 1c04, p. 24.

$b$ Neues Jahrb. f. Min. u. Geol., vol. 2, 1903, p. 69. 


\section{STATEMENT OF ANALYSES.}

Until recently it has been the practice in this laboratory to tabulate the constituents of a rock somewhat in the order of their determination, beginning with $\mathrm{SiO}_{2}$ as the chief constituent and grouping together all chemically related oxides, as shown, for instance, on page 17.

From a strictly scientific point of view a chemical classification founded on a separation into basic and acidic atoms or radicals would be more satisfactory, but until we learn to find out what silicic radicals are present and in what relative amounts, also how much free silica there may be, it is useless to think of employing the arrangement so valuable in stating water analyses.

Of late, petrographers have begun to demand, with considerable reason, an arrangement "which shall bring the essential chemical features-both the percentage figures and the molecular ratios-prominently and compactly before the eye, so that the general chemical character and the relations of the various constituents may be seen at a glance." $a$

In accordance with this demand it is now our practice to follow pretty closely the arrangement proposed by Pirsson and later strongly advocated by Washington, ${ }^{b}$ namely:

$\mathrm{SiO}_{2}, \mathrm{Al}_{2} \mathrm{O}_{3}, \mathrm{Fe}_{2} \mathrm{O}_{3}, \mathrm{FeO}, \mathrm{MgO}, \mathrm{CaO}, \mathrm{Na}_{2} \mathrm{O}, \mathrm{K}_{2} \mathrm{O}, \mathrm{H}_{2} \mathrm{O}$ (above 105$110^{\circ}$ ), $\mathrm{H}_{2} \mathrm{O}$ (below 105-110 $), \mathrm{CO}_{2}, \mathrm{TiO}_{2}, \mathrm{ZrO}_{2}, \mathrm{P}_{2} \mathrm{O}_{5}, \mathrm{SO}_{3}, \mathrm{Cl}, \mathrm{Fl}$, $\mathrm{S}\left(\mathrm{FeS}_{2}\right), \mathrm{Cr}_{2} \mathrm{O}_{3}, \mathrm{~V}_{2} \mathrm{O}_{3}, \mathrm{NiO}, \mathrm{CoO}, \mathrm{CuO}, \mathrm{MnO}, \mathrm{SrO}, \mathrm{BaO}, \mathrm{Li}_{2} \mathrm{O}, \mathrm{C}, \mathrm{NH}_{3}$.

$\mathrm{By}$ this arrangement the nine constituents which in the great majority of cases determine the character of the rock are placed at the head of the list, thus greatly facilitating the comparison of different analyses similarly arranged, especially when, as Washington recommends, the molecular ratios are calculated for these leading constituents and placed immediately after the corresponding oxides. The order of the remaining members is determined somewhat by the following considerations: $\mathrm{CO}_{2}$ is placed next after $\mathrm{H}_{2} \mathrm{O}$, since these two are generally a measure of the alteration the rock may have undergone. $\mathrm{TiO}_{2}$ and $\mathrm{ZrO}_{2}$ naturally follow $\mathrm{CO}_{2}$ on chemical grounds, and $\mathrm{SO}_{3}$ and $\mathrm{Cl}$, being common constituents of the sodalite group, are conveniently placed together.

It may be said with regard to the use of the word "trace" that the amount of a constituent thus indicated is supposed to be below the limit of quantitative determination in the amount of the sample taken for analysis. It should in general, for analyses laying claim to completeness and accuracy, be supposed to indicate less than 0.02 or even 0.01 per cent.

$a$ Washington, H. S., The statement of rock analyses: Am. Jour. Sci., 4 th ser., vol. 10, 1900, p. 61. $b$ Loc. cit. 


\section{TIME NEEDED FOR MAKING AN ANALYSIS.}

The question has often been put, "How long does it take to complete an analysis of this kind?". This will depend, of course, on the mineral complexity of the sample and on the personal factor of the individual worker. If there is a competent assist-

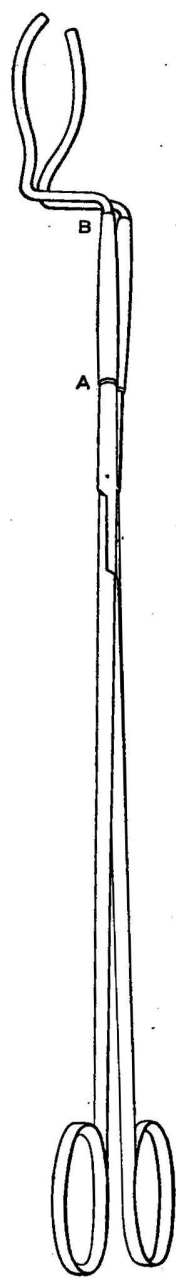

FIG. 1.-Platinum-tipped crucible tongs. The parts $A, B$, also of heavy platinum, are hollow, to serve as sockets for the cheaper metal of the handles. ant to do the grinding, and specific-gravity determinations are not required, it is quite possible after long experience for a quick worker to learn to so economize every moment of time in a working day of seven hours, with an abundance of platinum utensils and continuous use of air and water or steam baths through the night, as to finish every three days, after the completion of the first analysis, barring accidents and delays, one of a series of rocks of generally similar character, each containing from eighteen to twenty quantitatively determinable constituents excluding, for instance, fluorine, carbon as such, nitrogen, metals of the hydrogen sulphide group, and cobalt. But such an output of work implies an unusual freedom from those occasional setbacks to which every chemist is exposed.

\section{USEFUL APPLIANCES AND APPARATUS.}

In connection with the foregoing remarks it is in place to mention a few aids to the chemist which are in constant use in this laboratory and have come to be well-nigh indispensable. None is novel in principle and all are in use elsewhere, but some are not so commonly known as they deserve to be, hence this allusion to them. Certain cheap and simple forms of colorimeters have been found very useful, and since they are adapted to the determination of a variety of substances their description finds most appropriate place here.

\section{A. CRUCIBLE TONGS.}

Fig. 1 represents a form of platinum-tipped crucible tongs devised by Dr. A. A. Blair many years ago. With them a crucible can be securely grasped and brought into any desired position while still hot. To the contents, if in fusion over the blast flame, can be imparted the 
rotatory motion so often desirable. Above all, the cover need not be in the slightest degree displaced, as when using the common form of platinum-tipped tongs.

\section{B. RADIATORS FOR VOLATILIZING LIQUIDS AND SOLIDS.}

Fig. 2 represents a very useful adjunct to the worktable and especially to the draft cupboard, whereby the liquid contents of crucibles can be speedily evaporated at almost any desired temperature and the

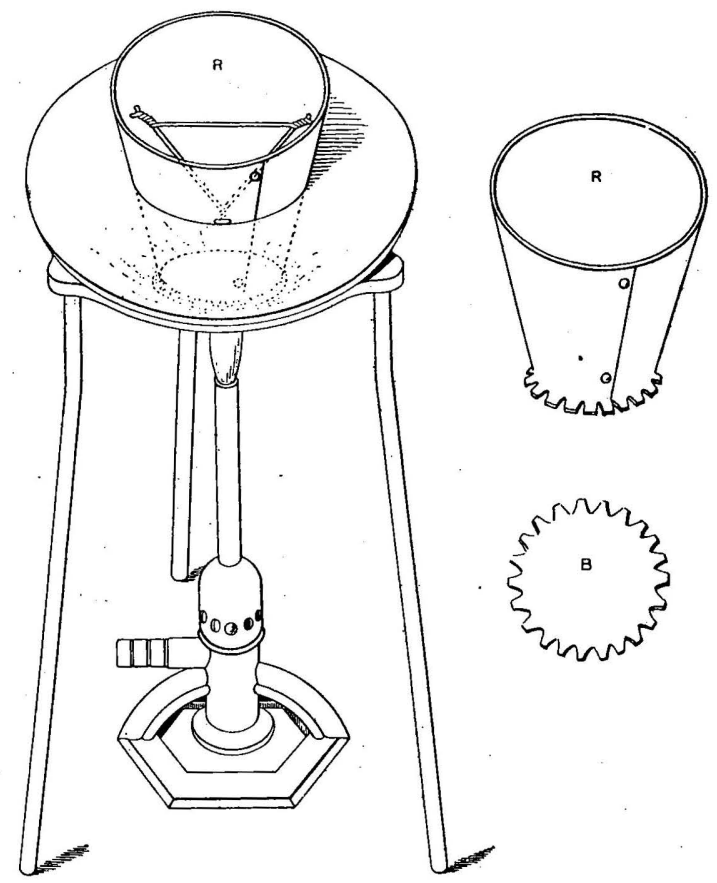

FIG. 2.-Radiator for rapid and safe evaporation. $R$ is of sheet iron or aluminum, also nickel (Jannasch). A convenient height is $7 \mathrm{~cm}$., width at top $7 \mathrm{~cm}$. and at bottom $5 \mathrm{~cm}$. The base, $B$, may be of iron, nickel, or platinum, but not aluminum, which will not stand the temperature of the direct flame. Platinum is most satisfactory by reason of its long life and radiating power. The manner of attaching $\mathrm{B}$ to $\mathrm{A}$ is by turning the cogs of $\mathrm{B}$ up and over those of $\mathrm{A}$. Evaporation may be greatly hastened when desired by placing upon A cast-iron ring with its opening somewhat larger than the mouth of the underlying crucible.

dehydration of many solids effected much more safely than on an iron plate or sand bath. I do not recall who originated this form of air bath, but it has been in use here for over twenty years and is identical in principle with the "Nickel-becher" of Jannasch. Nickel undoubtedly has a certain advantage in not rusting as does iron, but the form depicted in fig. 2 can easily be made anywhere of sheet iron riveted at the joint, the bottom being securely held by a notched flange at the extremity of the truncated cone. A crucible placed on the platinum triangle becomes uniformly heated by hot air, and large quantities of 
liquid, even sulphuric acid, can be thus volatilized in a short time without ebullition or spattering. The life and effectiveness of this appliance is greater if the bottom is of sheet platinum, but if an iron

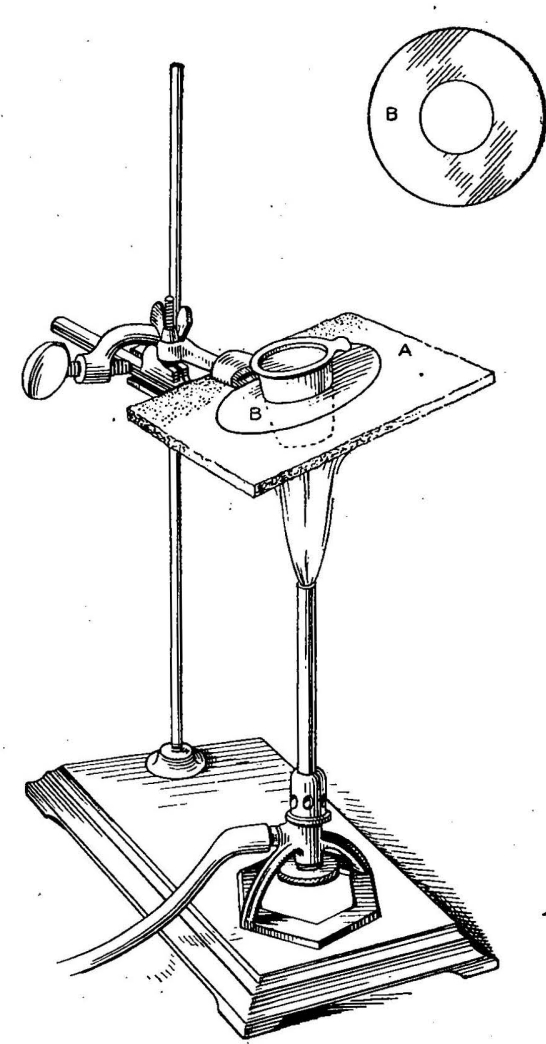

FIG. 3.-Device for excluding flame gases from the interior of crucibles during ignition. A, Asbestos board; B, stiff platinum foil of size suitable to the crucible used and having an opening to admit the crucible to about twothirds of its depth. This disk may be used alone upon the ring of an ordinary stand and in horizontal position, or, preferably as shown in the figure, in combination with the asbestos board, in which is an opening considerably larger than the crucible. The combination should then be given the inclined position shown, so as to allow the products of combustion to flow to one side without the possibility of their enveloping the mouth of the crucible. In the lack of platinum a perforated asbestos board alone will yield fair service with a blast, but not as a rule with an ordinary burner. desired to exclude flame gases from their interior, as in the ignition of ferric oxide and the determination of sulphur by fusion with an sible to replace it one or more times before the sides fail. A coating of aluminum paint on the sides adds to their life.

By an extension of the principle illustrated in fig. 2 very considerable amounts of ammonium salts and other easily volatile solids may be driven off from platinum dishes with little danger of loss by spattering and none by overheating. The dish containing the dry or nearly dry matter is placed in another of such size that the bottom of the inner dish is at some distance from that of the outer. The outer dish may be of iron, direct contact between it and the inner dish being prevented by wisps of asbestos overlapping the edge of the former, but the excellent conductivity and radiation of platinum recommends it above anything else. Aluminum will not stand the application of a full flame. If the contents of the dish are moist at first, a low flame will soon dry them, when the heat may be increased at will and the operation allowed to proceed without supervision. A sand bath may sometimes be substituted for the radiator.

\section{PERFORATED DISK FOR CRU- CIBLES.}

Fig. 3 shows an arrangement for the ignition of crucibles when it is bottom wears out it is usually pos(1)

(1)


alkali carbonate. The original idea of J. Löwe ${ }^{a}$ was to use perforated clay disks in determining the ash of coals. Later, Lunge and others advocated asbestos board, but this has the disadvantage that it lasts but a short time and the fibers adhere to the crucible to some extent. By neither of these is it easy to secure a very high temperature in the crucible. Therefore a disk of platinum is preferable because of its durability, cleanliness, and high conducting power, though its cost may preclude having a set to fit different sizes of crucibles. The platinum disk and asbestos board may be conveniently used in combination, as in the figure. The asbestos then has a large hole, over which a smaller perforated disk of platinum is laid.

\section{WEIGHING SCOOP OR TROUGH.}

Fig. 4 represents a most convenient receptacle for the sample that is being weighed on the balance. It is made of platinum and is counterpoised by a leaden weight. It has the advantage over a watch glass in that its shape permits of ready introduction of its contents into narrow-mouthed receptacles when desired.

\section{E. COLORIMETERS.}

a. SURVEY FORM.

The Survey form of colorimeter shown in fig. 5 consists of two glass reservoirs and a darkened box. The glasses, G, may be

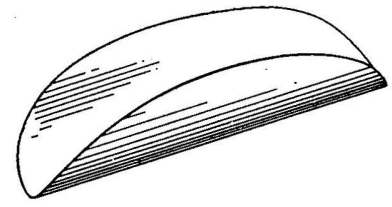

FIG. 4.-Weighing trough of platinum, to be balanced by counterpoise of lead. Length of trough about $7 \frac{1}{2} \mathrm{~cm}$; ; width before bending, $5 \mathrm{~cm}$. of square or rectangular section, 8 to $12 \mathrm{~cm}$. high and 3 to $3 \frac{1}{2} \mathrm{~cm}$. inside measurement between those sides through which the liquid is to be observed. ${ }^{b}$ These sides should, of course, be exactly parallel, the others need not be, but should be blackened externally. In order to exclude further the effect of side light, it is very convenient to have a simple light box, B, that can be easily held in the hand, stained black inside and out and with one end closed by a piece of ground glass, W, the other being open. For a space equal to the width of the glasses the cover is removed at the top next the glass end to permit the insertion of the glasses side by side in such a way that no light shall penetrate around their sides or between them. Immediately back of the glasses is a partition, $\mathrm{P}$, with openings of appropriate size

a Zeitschr. anal. Chemie, vol. 20, 1881, p. 224.

$b$ The allowable error in distance between the corresponding pairs of sides of the two glasses sinould not in any case exceed 1 per cent. Unfortunately there has been a disinclination on the part of dealers in this country to furnish glasses fulfilling this requirement, and held together by a durable cement which shall be proof against dilute sulphuric acid and alkali, though some have been obtained throvigh Messrs. Eimer \& Amend, of New York City. Canada balsam answers well for a time, but sooner or later it cracks, leaks then appear, and the sides soon drop off. It is, however, but a simple matter to cement them on again. But the use of Canada balsam is precluded with alkaline solutions, and hence for the chromium determination, as the solution at once becomes turbid. 
cut in it. A stiffly sliding black cardboard shutter, $\mathrm{S}$, is movable up and down immediately back of the partition, so that all light can be cut off except that which comes through the liquid. The ground-glass window, W, sliding up and down in slots, affords a much more uniform illumination than can be had without it.

Precautions of this kind are necessary if accurate results are to be counted on. Except for mere traces, this combination of glasses and darkened box insures greater accuracy and rapidity of work than Nessler tubes, and is preferable likewise, so far as my experience goes, to expensive instruments like the colorimeter of Soleil-Duboscq, etc.

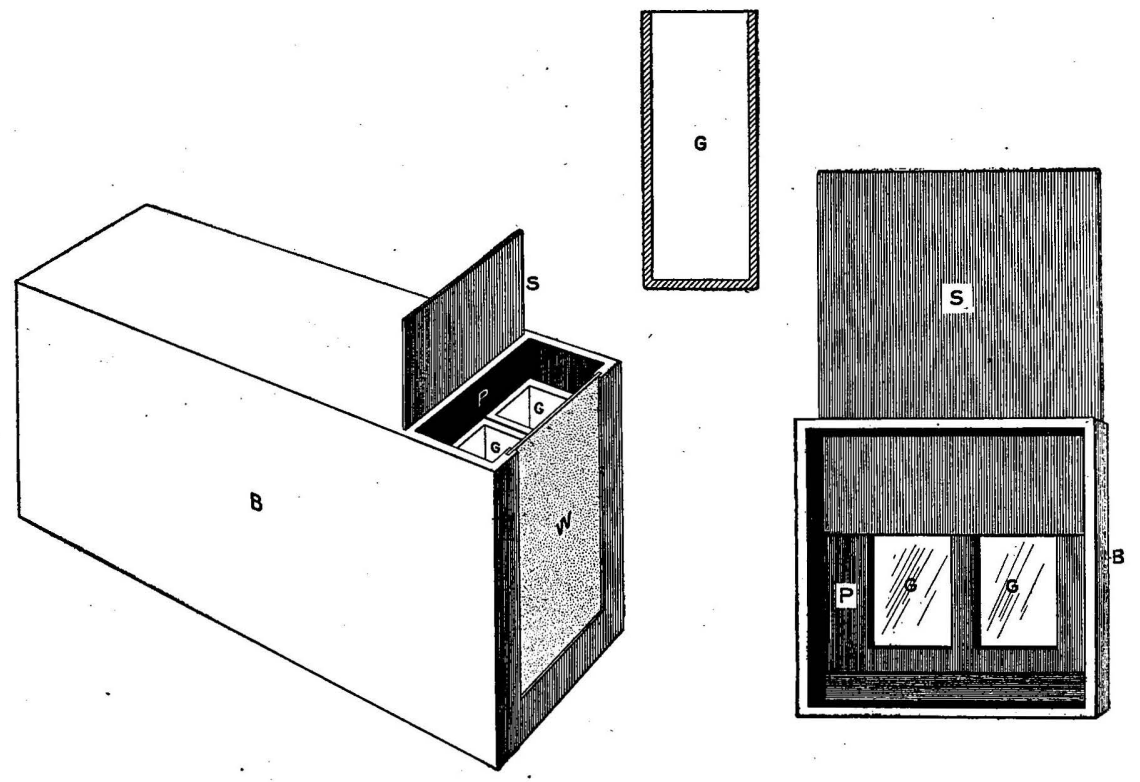

FrG. 5.-Survey form of apparatus for colorimetric determinations, in different aspects. G, One of two glasses of square or rectangular section, 8 to $12 \mathrm{~cm}$. high and 3 to $3 \frac{1}{2} \mathrm{~cm}$. inside measurement between those sides through which the liquid is to be observed. The other sides are blackened on the outside. B, Rectangular box about $35 \mathrm{~cm}$. long and $12 \mathrm{~cm}$. square, stained black inside and out, one end closed by a ground-glass window, W, the other open, and a portion of the top removed. P, Blackened partition, with openings corresponding to the interior dimensions of the glasses when in position. S, Blackened cardboard shutter sliding stiffly up and down back of the partiticn.

The manner of using the colorimeter is as follows: Any suitabie amount of the standard solution, which is purposely made stronger than the solution to be tested, is placed in one of the glasses and an indefinite part or the whole of the test solution in the other. Water is then added from a burette to the standard until there is no distinction as to color. Perfect mixture is brought about by a glass rod flattened at one end. In making the color comparison the box is best held close to a window, so as to get a full, strong light. Daylight is far preferable to artificial light. The amounts of the substance to be determined and that in the standard cylinder are of course proportional to the volumes of the matched solutions. 
b. SCHREINER'S FORM.

Dr. Oswald Schreiner, of the United States Department of Agriculture, has devised a colorimeter (fig. 6), ${ }^{a}$ working on the same basic principle as the far more expensive ore of Soleil-Duboscq, which has done good service in colorimetric work on phosphates, etc. Its description, personally communicated by Doctor Schreiner, is as follows:

The colorimeter in its simplest form consists essentially of graduated glass tubes, $\mathrm{B}$, containing the standard and the unknown colorimetric solution, the column of the liquid in both tubes being changed by means of two smaller immersion tubes, $A$, also of glass. The upper ends of the tubes $A$ are mounted in blocks of wood which fit into grooves on the body of the colorimeter. This permits the ready removal of the tubes from the colorimeter for cleaning when the liquids are changed. The graduated tubes, B, are supported by a block of wood about midway of the camera and are held in place by brass springs or clamps $\mathrm{C}$, the tension of which can be regulated to suit the diameter of the tube, so as to allow it to be moved freely up or down by hand and yet be firmly held in position when the setting is made. It will be noticed from the figure that the glass tubes diverge slightly. This has been found of advantage, as it allows the operator to look down the center of both immersion tubes, as well as to bring the upper parts of the tubes, and consequently the images closer together. The openings to the immersion tubes can be covered by means of a microscope slide to prevent dust from falling into the tubes. The tubes are illuminated from the reflector, $\mathrm{D}$, below, which carries a white sheet of cardboard or opal glass, and after passing through the tubes the light is reflected by a mirror, E, in the upper part of the instru-

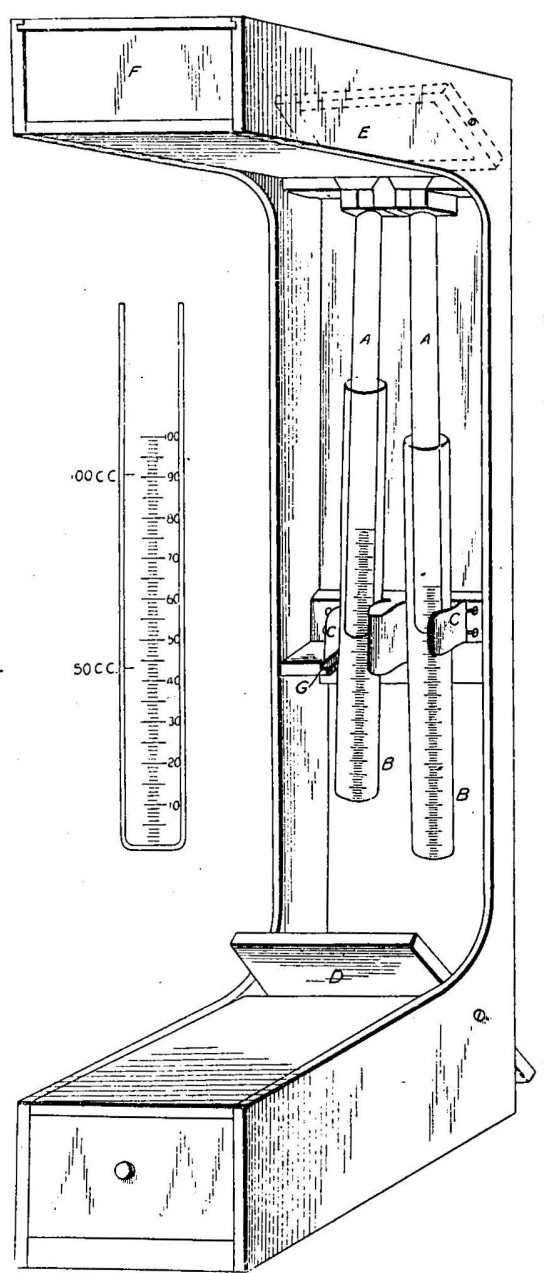

FIG. 6.-Schreiner's colorimeter. ment to the eye of the observer at $F$. The dimensions of the camera are about 70 by 32 by $16 \mathrm{~cm}$. Standard glass slides may be inserted at $\mathrm{G}$ below one of the immersion tubes in place of the tube containing the standard solution, the immersion tube being retained so as to give similar images when viewed from above.

The measuring colorimeter tubes, $\mathrm{B}$, are $25 \mathrm{~cm}$. long, with inside diameter as nearly as practicable $27 \mathrm{~mm}$.; in no case less than $25 \mathrm{~mm}$. and not greater than $29 \mathrm{~mm}$. The glass should be colorless and the bottoms well ground and polished, with internal surfaces of the bottoms plain, in no case appreciably convex or concave. The bottoms must be ground

a Jour. Am. Chem. Soc., vol. 27, 1905, p. 1192.

Bull. $305-07-3$ 
down sufficiently to make the ground surfaces a little larger than the internal diameters of the tubes; each of the tubess is provided with an etched scale of one hundred 2-mm. divisions. The scale begins at the level of the inner surface at the bottom, the length of the marks being $6 \mathrm{~mm}$. and every fifth mark $12 \mathrm{~mm}$., and every tenth mark numbered on the right side, 10,20 , 30, etc., beginning at the bottom. On the reverse side of each measuring tube may be etched two capacity marks, if desired, one for $50 \mathrm{~cm} .^{3}$, the other for $100 \mathrm{~cm} .{ }^{3}$

The smaller tubes are likewise $25 \mathrm{~cm}$. long, with outside diameter not greater thin $20 \mathrm{~mm}$. and not less than $18 \mathrm{~mm}$., with the thickness of the glass the same in all the tubes. The bottoms of these tubes must be carefully ground and polished and the inside of the bottom never sensibly convex or concave. They must be of colorless glass with the bottoms so ground that the diameter of the ground portion exceeds the internal diameter of the tube. These tubes are to be provided with neither scale nor capacity marks.

The standard colorimetric solution is poured into one of the graduated tubes and put into place in the camera together with the immersion tube. The unknown colorimetric solution, made up to definite volume, is put into the other graduated tube and similarly placed in the instrument. The tube containing the solution of unknown strength is set at a convenient height, say fifty scale divisions, and the other tube containing the standard moved up or down, the operator watching the effect on the image in the mirror through the opening in the front of the camera. By moving the tube so that the image is alternately weaker or stronger than the standard, the setting can be accurately and quickly made. When both images show the same intensity of color, the setting is read by noting the division mark on the graduated tube opposite the ground bottom of the immersion tube. This reading will give the height of column of the standard which exactly corresponds with the intensity of color of the column of the unknown solution. It follows that the strengths of these two colorimetric solutions are inversely as the heights of the columnsthat is, as the readings on the two tubes. If $\mathrm{R}$ is the reading of the standard solution of strength $\mathrm{S}$, and $r$ the reading of the colorimetric solution of unknown strength $s$, then $s=\frac{\mathrm{R}}{r} \mathrm{~S} .{ }^{*}$ The same formula, of course, applies when the standard is fixed and the unknown solution moved up or down until the images are of equal intensity.

\section{PRELIMINARY QUALITATIVE ANALYSIS.}

A complete qualitative analysis of a rock, preceding the quantitative examination, is in most cases a sheer waste of time. A few constituents may now and then be specially looked for, but in general time is saved by assuming the presence of most of them and proceeding on that assumption in the quantitative analysis.

This statement must not be misinterpreted, as has occurred, so as to convey the impression that qualitative analysis receives little consideration in the chemical work of the Survey. For ores, mineral species, waters, etc., it is indispensable, as also in the testing of precipitates and filtrates during the course of quantitative analysis.

\section{QUALITY OF REAGENTS.}

Unquestionably the greatest hindrance to good work in otherwise well-equipped laboratories is the difficulty in securing satisfactory reagents. This fact early became apparent to the Survey chemists, and it is mainly to their strenuous complaints and initiative that the present movement in favor of better quality is due, Much of 
the glassware on the market is of a very inferior grade and utterly unsuited for analytical work.

All analyses performed in the Survey laboratory have been made with the purest reagents obtainable, either by purchase in the open market or by special preparation on the part of manufacturers or in the laboratory, but the most ceaseless vigilance has at all times to be practiced. As is now generally known, a "C. P." label is no guaranty whatever of the purity of a reagent. Every new purchase must be examined if it is one in which purity is a desideratum. In general, all so-called "C. P." chemicals should at least stand the tests laid down by Krauch. ${ }^{a}$ The "guaranteed" reagents have been found at times to be worse than those emanating from sources which make no claim to special purity for their goods.

Acids made in this country can be had of a high grade, and these need no redistillation except for special experiments.

Hydrofluoric acid had to be always freshly distilled with potassium permanganate until the introduction of ceresin bottles afforded an article sufficiently pure for the most exacting work. Care must be taken to see that no particles of paraffin or ceresin are floating on the acid as it is poured out, and that this is free from traces of hydrochloric acid whenever it is to be used for attacking silicates with a view to determining chlorine (C. b, p. 155). An important use for hydrofluoric acid is the decomposition of refractory silicates in the determination of ferrous iron. For this purpose it is necessary that it be free from any substance that will reduce permanganate. Sulphurous acid was found in one or two shipments, but this is a contamination against which care in manufacture can easily guard.

Ammonia ought always to be redistilled at short intervals, after first shaking up with slaked lime in order to decompose any ammonium carbonate that it may hold. Glass stock bottles may be coated inside with ceresin so as to prevent contact between the glass and the ammoniacal solution, a device that may be serviceable with other reagents.

Potassium bisulphate should be entirely free from silica, alumina, and heavy metals. The only correct way to get an accurate silica determination is to fuse the salt with a considerable amount of strong sulphuric acid, allow to cool, dissolve in water, and digest on the steam bath to coagulate the silica, which is then filtered; or, the salt may be dissolved in water and evaporated with a large excess of sulphuric acid till fumes of the latter rise copiously. On cooling, the mass is dissolved and treated as above. If a satisfactory article is not to be purchased, it can be made from equivalent parts of potassium sulphate and sulphuric acid. Even then the normal salt has first to be examined, for it has been found to contain

$a$ Die Prúfung der chemischen Reagentien, 31 ed., Berlin, Julius Springer, 1896. English translation by J. A. Williamson, D. Van Nostrand Co., 1902. 
notable amounts of lead, calcium, and silica. Sodium bisulphate is for some purposes preferable to the potassium salt. Both should, however, be converted to pyrosulphate by fusing in a large platinum dish and maintaining in fusion till all spattering has ceased and sulphuric acid rises copiously. The salt thus treated will not show the disagreeable frothing and spattering that is so objectionable when making fusions with the bisulphate and it is more quickly effective.

The phosphorus salt used for precipitating magnesium has been found to contain iron and silicon.

Ammonium oxakate almost always contains calcium, and must therefore be purified or specially prepared. The same may be said of oxalic acid. According to A. Classen, ${ }^{a}$ ammonium oxalate sometimes contains lead.

Ammonium chloride has been known to contain manganese.

Hydrogen peroxide sometimes contains fluorine, which renders it unfit for use as a chemical reagent. The test for fluorine is carried out as follows: To $50 \mathrm{~cm} .^{3}$ of the reagent add a slight excess of sodium carbonate and warm the solution. Filter if a precipitate forms, and to the boiling solution add calcium chloride in excess. Filter and gently ignite the precipitate. Treat it with dilute acetic acid, drop by drop, till the calcium carbonate is dissolved, filter, wash, gently ignite the residue, and test it with strong sulphuric acid in the usual way for fluorine. The perhydrol of E. Merck, now on the market, is a very pure and strong peroxide.

Sodium carbonate, one of the most important reagents in silicate analysis, can be had which does not carry more than $2 \frac{1}{2}$ milligrams of total impurity (see $\beta$, p. 72) in 20 grams (0.012 per cent). This should be reserved for the main portions, in which silica, alumina, etc., are to be determined. For other constituents, as phosphoric acid, fluorine, sulphur, a poorer grade is quite allowable, provided it is free from the element to be determined and from any other which might interfere with its determination Silica is to be tested for by evaporating the solution of the carbonate or the dry salt with sulphuric acid in large excess till fumes of the acid are copiously evolved, dissolving the cooled mass in water, and digesting on the steam bath in order to coagulate the silica before filtering. Evaporation with hydrochloric acid will not yield all of the silica. Not long ago a very objectionable impurity in an otherwise acceptable article was encountered, namely, fluorine. The explanation of its presence is probably that cryolite had been used as the source of the carbonate. Sodium phosphate in small amounts seems to be a common contaminant of even good grades of sodium carbonate.

Sodium hydroxide made from sodium has been found with a large amount of lead, a fatal obstacle to successful mineral analysis, and also with small amounts of nickel. 
Owing to the solvent action on glass of many solutions of solid reagents, these solutions should be made up at frequent intervals in limited quantities, or, preferably, the solid should be dissolved as wanted. That this is no unnecessary refinement in really good work has been amply demonstrated, and it is particularly called for with such reagents as ammonium oxalate and microcosmic.salt.

\section{PART II.-METHODS APPLICABLE TO SILICATE ROCKS.}

\section{INTRODUCTORY REMARKS.}

The order hereinafter followed in describing the various chemical separations has little relation to the affinities of the constituents of the rock, but those are grouped together which can be conveniently determined in the same portion of rock powder. Thus, in the main portion are usually determined $\mathrm{SiO}_{2}, \mathrm{TiO}_{2}, \mathrm{MnO}, \mathrm{NiO}, \mathrm{CaO}, \mathrm{SrO}, \mathrm{MgO}$, total iron, and the combined weight of all the following: $\mathrm{Al}_{2} \mathrm{O}_{3}, \mathrm{TiO}_{2}, \mathrm{P}_{2} \mathrm{O}_{5}, \mathrm{ZrO}_{2}$, all iron as $\mathrm{Fe}_{2} \mathrm{O}_{3}$, and nearly if not quite all vanadium, as $\mathrm{V}_{2} \mathrm{O}_{5}$, also perhaps rare earths if present. In a separate portion is estimated $\mathrm{FeO}$, and also the total iron, as well as $\mathrm{BaO}$, if these last are desired as checks. The alkalies need a portion for themselves. In another, $\mathrm{ZrO}_{2}, \mathrm{BaO}$, rare earths, and total sulphur are very conveniently determined. For $\mathrm{V}_{2} \mathrm{O}_{3}$ and $\mathrm{Cr}_{2} \mathrm{O}_{3}$ still another and usually much larger portion is to be used. Determinations of $\mathrm{CO}_{2}, \mathrm{C}, \mathrm{H}_{2} \mathrm{O}, \mathrm{Fl}, \mathrm{Cl}$ are all best made in separate portions of substance, tho various combinations are possible, as $\mathrm{CO}_{2}$ and $\mathrm{H}_{2} \mathrm{O}, \mathrm{C}$ and $\mathrm{H}_{2} \mathrm{O}$, or $\mathrm{H}_{2} \mathrm{O}, \mathrm{Fl}$, and $\mathrm{Cl}$. In fact, by a judicious selection and combination of methods a very satisfactory analysis can sometimes be made on 4 grams of material without omission of anything of importance, though the time consumed will be greater than if ample material is available.

As an illustration of the advantage to be gained by a little judgment in the combination of methods, the case of sulphur, barium, zirconium, and rare earths may serve. Many chemists never look for any but the first of these, but by following the procedure given under A, page 115, not much more labor is expended in confirming their presence or absence than that of sulphur alone.

With only occasional exceptions nearly all the constituents mentioned on page 21 can be determined if present in portions of powder not exceeding 1 gram each in weight.

This is a convenient weight to take for the main portion in which silica, alumina, etc., the alkaline earths, and magnesia are to be sought; but it should, in general, be a maximum, because if larger the precipitate of alumina, etc., is apt to be unwieldy. Its weight can not often be much reduced with safety if satisfactory determinations. of manganese, nickel, and strontium are to be expected. For the alkali 
portion one-half gram is a very convenient weight. In general, it may be made a rule not to use more than 2 grams for any portion which has to be fused with an alkali carbonate, as for sulphur, fluorine, and chlorine. For carbon dioxide the weight may rise to 5 grams, or even more, if the amount of this constituent is very small, without expenditure of any more time than is required by 1 gram and with correspondingly greater approach to correctness in the result. For vanadium also a larger weight than 2 grams is usually demanded.

For the attainment of accurate results such as are needed for the close classification of a rock in the system devised by Cross, Iddings, Pirsson, and Washington, ${ }^{a}$ too great stress can not be laid on the exercise of the utmost care throughout the analysis. This applies not only to routine manipulations, but even more in the matter of double precipitations, where these are called for, and in the testing of filtrates and precipitates to insure complete recovery of the desired substances, on the one hand, and freedom of them when obtained from matter which should not to be there, on the other. These cautions are especially urgent in the case of those having little experience. It must always be remembered that analysis can at best afford but approximate results, which will be the more serviceable the greater the care bestowed in obtaining them.

\section{SPECIFIC GRAVITY. (POROSITY.)}

GENERAL REMARKS.

Though a knowledge of the specific gravity of rocks is seemingly less prized now than formerly by petrographers, it is occasionally needed and is ever a most important criterion in the identification of mineral species. As ordinarily carried out, whether by the method of suspension or by the pycnometer, the results fall short of the accuracy which is generally credited to them. A really accurate determination of the specific gravity of solids.by the pycnometer, in which the error shall not exceed one or two points in the third decimal, is to be achieved only with the most painstaking precautions (see B. b, p.43). Without this care the error in the second decimal may, and often does, amount to several points. For these reasons the subject of specific gravity is treated in some fullness. Although the porosity of a rock is a constant seldom used by the petrographer, it is one that is most useful in the comparative study of building stones, and since its determination as often carried out is likewise faulty and gives a value which does not represent the pore space in terms of the volume of stone, it has been deemed expedient to devote space to this subject also, especially as the determination of porosity involves that of specific gravity. 


\section{A. BY SUSPENSION IN WATER.}

a. ORDINARY METHOD FOR SPECIFIC GRAVITY.

This determination, when required, is best made on one or several fragments weighing up to 20 grams. They are held together by a fine platinum wire ready for suspension from the balance, and thus held are placed in a small beaker to soak over night in distilled water under the exhausted receiver of an air pump side by side with a similar beaker of water. Boiling is, of course, a much less effective means of removing air than the air pump, and the boiling water may exert an undesirable solvent and abrading effect. In the morning the wire is attached to the balance arm, the rock fragments remaining immersed in the water; a thermometer is placed in the companion beaker of water, now likewise in the balance case, and the weight is at once taken. Both vessels of water having precisely the same temperature, it is quite unnecessary to wait for the water to assume that of the balance should it not already possess it. The fragments are now lifted out, without touching the vessel, and carefully transferred to a tared crucible or dish. The wire is removed and at once reweighed, with the precaution that it dips just as far into the water now as when weighted. Hereby a special weighing of the wire out of water is avoided. The sample may now be dried on the water bath and then at $110^{\circ}$ for some hours to certainly expel all absorbed water, and weighed after prolonged cooling in the desiccator. It is better to ascertain the weight of the dry rock after soaking in water than before, in order to avoid the error due to possible breaking off of a few grains between the two weighings. Should the density of the rock in air-dry condition be required, it may be left exposed to the air for a long period after drying and before weighing; ${ }^{a}$ but the difference will only

\footnotetext{
$a$ In view of the uncertainty as to what constitutes hygroscopic water (see e, p. 53), this course is perhaps
} more to be commended than the former, and seems imperative for certain zeolitic rocks. In such cases it is best to weigh the fragments before putting to soak, and afterwards to collect on a Gooch crucible the grains which may have fallen off in the water. Should no crucible of this kind be available, a paper filter may unhesitatıngly be used and incinerated with the powder, owing to the small amount of which the error due to loss of even all its water during ignition is quite negligible.

Since the first publication of the foregoing in Bulletin No. 143, a series of determinations on eight rocks, in which the weighing was done after drying. led to an interesting observation. The lumps were heated for a short time on the steam bath until dry on the surface, then allowed to cool in air and weighed from time to time during two or three days to constant weight. It was observed that some lost weight from the start, while others gained. For instance:

$\begin{array}{rrr}14.3803 & 9.5342 & 17.0846 \\ .3730 & .5178 & .1089 \\ .3666 & .5121 & .1159 \\ .3483 & .5024 & .1413 \\ .3481 & .5016 & .1388 \\ .3450 & .5016 & .1379 \\ 3457 & & \end{array}$

The losses in the first and second cases may reasonably be ascribed to the gradual evaporaition of water absorbed in the pores, while the increase in the third case is doubtless indicative nf the presence of zeolitic constituents, from which even brief exposure to the heat of the steam bath had sufficed to drive out a good deal of their loosely held water. Subsequent careful analysis confirmed this view. for each rock that gained in weight was found to hold a relatively large amount of water removable at $105^{\circ}$, and the microscope revealed the presence of zeolitization in them and not in the ofhers. Hence, this procedure may be sometimes of value as a test for the presence of zeolitic minerals in advance of or as confirmation of the microscopic diagnosis. 
in exceptional cases affect the second decimal by more than a single unit. For instance, an undried rock of 2.775 specific gravity containing in the uncrushed state the high percentage of 0.3 hygroscopic moisture will have a density of 2.79 when dry; a rock of 2.982 specific gravity, undried, will have a density of 3.00 after removal of 0.3 per cent of moisture. The difference becomes greater as the density of the rock increases.

This method of ascertaining the specific gravity of rocks is certainly more convenient than, and for compact rocks is believed to be decidedly preferable to, that of the pycnometer, in which the fragments must be reduced to small size with consequent formation of more or less powder, which is subject to slight loss. in the various manipulations. To exclude this powder and employ only small fragments would introduce a possible source of error, since it is likely to consist largely of the most easily abraded minerals and consequently not to have the average composition of the mass. By following the instructions given above, loss of material is absolutely avoided, a decided saving in time is effected, and considerable weights can be easily employed with consequent. lower probable error in the results. To vesicular rocks, however, notably certain lavas, the above procedure is, of course, inapplicable, since it is probable that many of the interior pores would be inaccessible to penetrating water.

b. METHOD FOR SPECIFIC GRAVITY AND POROSITY COMBINED.

E. R. Buckley ${ }^{a}$ has called deserved attention to the faultiness of the methods generally employed for the determinations of these constants as applied to building stones, and has brought together in the work cited the fruits of his experience. His recommendations are essentially as follows:

The specimens should be cubes, such as are commonly employed for the other physical tests, measuring usually 2 inches square. These are dried for at least twenty-four hours at $110^{\circ}\left(100^{\circ}\right.$ is not high enough to insure the removal of all interstitial water) and weighed to centigrams. They are then placed on the bottom of a larce bottle, the cork of which can be hermetically sealed. Through the cork pass three tubes, two of which end below the cork and connect with a vacuum pump and manometer, respectively. The third tube, provided with a stopcock, ends near the bottom of the bottle and leads into a basin of water which can be heated to boiling. The bottle itself rests in a water bath, so that its contents can be kept hot all the time. At first the cubes rest in a shallow layer of water only, the better to facilitate the escape of air and entrance of water under reduced pressure, which should not exceed one-twelfth of an atmosphere. The cubes should remain in this state for seventy-two hours,

$a$ Building and ornamental stones of Wisconsin: Bull. Wisconsin Geol. Nat. Hist. Survey No. 4, 183s, pp. 63, 70 . 
experiment having shown that little or no absorption takes place after this lapse of time. Water is slowly let in from time to time, till the cubes are wholly immersed. In this way it is thought that thorough saturation can be attained. The saturated samples are transferred to an open dish filled with water and taken to the balance, where their weight in water is ascertained by suspension. They are then removed from the water, rapidly dried on the surface with bibulous paper, and the weights then taken in air. ${ }^{a}$ They are dried at $110^{\circ}$ for twentyfour hours and again weighed. The second dry weight was found by Doctor Buckley to vary rarely from the first by as much as 0.2 gram, and often by not more than 0.01 . "When reckoned in per cent, the error would be such a small fraction of 1 per cent as not to be worthy of attention. The specific gravity was determined by dividing the average of the two dry weights by the difference between the dry weight and the weight of the cube suspended in water." For arriving at the true porosity or actual pore space compared with the volume of the sample tested, the following formula is to be used:

$$
\frac{(B-A) \text { sp. gr. } 100}{(B-A) \text { sp. gr. }+\mathrm{A}}=\mathrm{P}
$$

in which $\mathrm{A}$ is the average dry weight, $\mathrm{B}$ the saturated weight, and $\mathrm{P}$ the percentage of porosity or actual pore space. The value thus obtained is always much higher than that ordinarily taken as the measure of porosity. This last would better be called, according to Doctor Buckley, "ratio of absorption," being found by dividing the weight of water absorbed by the dry weight of the stone and multiplying by 100. Doctor Buckley finds no definite relation between porosity and ratio of absorption, although in a general way the former is a little more than twice the latter.

In calculating the weight of a stone in pounds per cubic foot the pores should be included in this volume. The weight sought is found by multiplying the apparent specific gravity by 62.5 - that is, by the weight of a cubic foot of water. The apparent specific gravity can be calculated from the above data by the formula

$$
\frac{\mathrm{A}}{\mathrm{B}-\mathrm{C}},
$$

in which $\mathrm{A}$ is the dry weight, $\mathrm{B}$ the saturated weight, and $\mathrm{C}$ the weight in water.

$a$ The above procedure differs from that of Doctor Buckley in the inversion of the order of weighing in water and after wiping. The more perfect the vacuum the less need be the temperature of the bath of immersion to insure the effective aid, by boiling of the water drawn up into the pores of the specimen, in securing expussion of the inclosed air. A vacuum desiccator can perhaps be substituted for the bottle with much advantage. 1t will not be necessary to open the desiccator for the periodic introduction of water if to the evacuating tube is attached a $\mathrm{Y}$ or $\mathrm{T}$ with stopcocks, one branch leading to the pump and manometer, the other to a basin of water. By proper regulation of the cocks water can be drawn in at pleasure. In a later publication (Missouri Bureau Geol. and Mines, 2d ser., vol. 2, 1904) Doctor Buckley states that he has reduced the time of immersion to twenty-four hours and the vacuum to onefourth of an atmosphere, without finding appreciable differences in the results obtained. 
T. W. Thörner ${ }^{a}$ and H. Seger and E. Cramer ${ }^{b}$ have described apparatus alike in principle for determining porosity by the use of which a direct specific-gravity determination is avoided and the operations are very simple. The apparatus of Seger and Cramer are of simpler construction than those of Thörner and have stood the test of many years' use. By both, however, the true porosity as well as the "ratio of absorption" is ascertainable.

c. PENFIELD'S METHOD FOR MINERAL FRAGMENTS.

Penfield ${ }^{c}$ recommends the following modification of the suspension method as more convenient than that by the pycnometer in many cases for small fragments of minerals.

After boiling in water, the substance is transferred with water to a small glass tube about 8 by $35 \mathrm{~mm}$., provided with a fine platinum wire for suspension. This is weighed full of water in another vessel of water, and again after the removal of the mineral, the weight of which is found after drying.

This method is, of course, more applicable to homogeneous minerals than to rock fragments, and will therefore be applied in rock analysis chiefly to the determination of the specific gravity of the mineral grains separated by heavy solutions or acids.

\section{B. PYCNOMETER METHOD.}

a. ORDINARY METHOD.

If the pycnometer has to be used, as is generally the case when the density of any one of the mineral ingredients of a rock is desired after separation by one of the approved methods, it being then in a more or less finely divided state, a good procedure is that adopted in this laboratory by L. G. Eakins a number of years ago, provided there is no evaporation whatsoever around the stopper, a condition seldom, if ever, fulfilled, unless a thin film of vaseline is used, the weight of which need not exceed a half milligram. The pycnometer used is one with a capillary stopper, provided with a millimeter scale etched in the glass, the divisions being numbered both ways from the center and calibrated by mercury, so that the value of each one in weight of water is known. The capacity of the flask filled with water to the zero division is then calculated for every half degree of temperature from $0^{\circ}$ to $30^{\circ}$ by making a series of careful weighings in which, the capacity of the stem being known, it is quite immaterial at what level the water stands, provided it is within the limits of the scale. The exact temperature is obtained by an accurate thermometer placed in a companion vessel of similar shape to the pycnometer and containing 
a like amount of water, both being left in the balance case till its temperature has been nearly or quite assumed, as shown by a second thermometer. The weighing must of course be made before the thread of water has sunk beneath the lowest division, which it will do after a time, even though at first filling the bore to the top of the stopper; and the corrected weight full of water to the zero mark is found by adding or subtracting the needed amount, as shown by the height of the thread on the scale.

For each pycnometer in use, and these are of different sizes, is prepared a table showing its weight, the value of each scale division in grams of water, and the capacity of the flask at different temperatures, as indicated above. The preparation of such a series of flasks is time saved in the end, for the weighing of the flask full of water each time a density determination is made is rendered superfluous. All that is necessary is to look up in the table the weight corresponding to the temperature.

The density of the previously weighed substance in this case is now determined in much the same way as in A. a, p. 39, after the unstoppered pycnometer containing it and nearly filled with water has stood with its companion vessel of water under the air pump the necessary length of time. The water needed to fill the flask is taken from its companion.

All who have used the pycnometer method for fine substances know the difficulty experienced in preventing a portion from being held at the surface, despite all attempts at making it sink. Hence it often happens that a very small portion runs out around the sides of the stopper on inserting it. If the flask rests in a small tared dish the grains thus forced out may be washed down into it and weighed after evaporation in order to get the correct weight of that in the flask; or; after weighing, the contents of the flask may be emptied into a tared dish and the water slowly evaporated off in order to get the weight of the mineral. Usually this way is less to be recommended than the other.

\section{b. REFINED METHOD.}

In their elaborate study of the thermal properties of the feldspars ${ }^{a}$ Drs. A. L. Day and E. T. Allen found the ordinary methods of specific-gravity determination of powders by the pycnometer to be affected with grave errors. In the following is given their procedure, slightly modified since publication, for obviating them to such a degree that with $25 \mathrm{~cm} \cdot{ }^{3}$ pycnometers and 5 to 10 grams of material of from 2.50 to 2.75 specific gravity the error from all causes should never be greater than one unit $( \pm 1)$ in the third decimal place.

$a$ Pub. Carnegie Institution of Washington, No. 31; and in less detail in Am. Jour. Sci.; 4th ser.; vol. 19, 1905, p. 93 . 
A chief source of error is the evaporation of water around the stopper. In order to reduce this to a negligible quantity, the grinding should be very fine and the stopper should be slightly vaselined before the final weighing. If the excess of vaseline is carefully wiped off, the error due to its weight will not affect the result in the third decimal with $25 \mathrm{~cm} .{ }^{3}$ flasks. The best flasks are those with capillary stoppers, and Day and Allen now have them made with necks $2 \mathrm{~mm}$. thick, so as to avoid springing when the stopper is pressed in, and ground square off to prevent the adhesion of a film of water between stopper and neck above the joint. With the stopper slightly vaselined, such a flask does not lose at $30^{\circ}$ more than 1 to $1 \frac{1}{2}$ milligrams per half hour.

To obviate another serious source of error, incomplete removal of air from the powder and undue loss of powder during filling and stop-

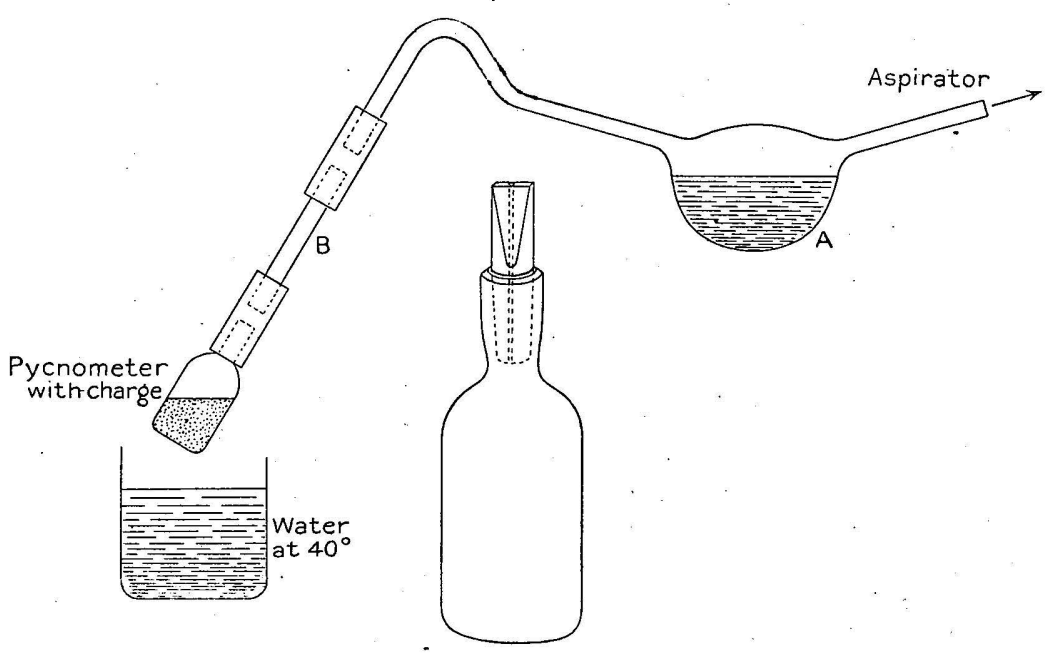

Fig. 7.-Moore's device to remove air from mineral powders.

pering, the device of G. E. Moore ${ }^{a}$ is made use of, slightly modified, as shown in fig. 7. The flask containing the powder is attached to a water pump, with the intervention of a tube and bulb attachment, as shown. The bulb A contains boiled water. When the system has been nearly freed from air by the pump, water from $A$ is made to flow into the flask. Then by tapping and plunging the latter into water warmed to $40^{\circ}$ to $50^{\circ}$, in order to produce boiling within the flask, the air is soon removed. During the boiling powder will usually be thrown up into the connecting tube; but this can be washed back hy water from A or, after disconnecting, by boiled water from a wash bottle. Should any particles remain in the tube, they are washed into a tared dish and weighed, after evaporation of the water. It is absolutely essential that not the smallest grain be caught between the

$a$ Am. Jour. Sci., 3 d ser., vol. 3, 1872, p. 41; Jour. prakt. Chemie., vol. 2, 1870, p. 329. 
stopper and the neck. To this end the neck is wiped out with a bit of filter paper, which is burned in the tared dish in order to obtain the proper correction.

Constant temperature is attained in a water thermostat electrically regulated to tenths of a degree at a temperature slightly above that of the balance case. In the thermostat there is also placed a small vessel with water for the final filling. If the powder is very fine, the flask may have to remain in the thermostat several hours to allow complete settling before wiping the neck, vaselining, and stoppering. When filled and stoppered, it is advisable to return it to the thermostat for a time, with a short piece of rubber tube slipped part way over the end of the stopper, so as to form a cup for a drop of water and insure that the capillary remains filled. At the proper time the rubber is removed and the flask quickly wiped with bibulous paper and weighed without delay. A blank test must of course be made with water alone, or the pycnometer may be calibrated once for all within any desired range of temperature. A determination of the accuracy attainable in this way is subject to a correction for buoyancy.

(See p. 55 for remarks by Doctors Day and Allen on another source of error in the determination of the specific gravity of powders.)

\section{HEAVY SOLUTIONS NOT SUITABLE FOR ROCKS.}

Because of their roughness, porosity, and complex mineral composition the density of rock fragments can not be accurately determined by that of heavy solutions in which they may remain suspended.

\section{PREPARATION OF SAMPLE FOR ANALYSIS.}

A. QUANTITY OF ROCK TO BE CRUSHED.

In the great majority of cases a few chips from a hand specimen will well represent the average of the mass, but with rocks in which a porphyritic structure is strongly developed the case is different. Here a large sample should be provided, gaged according to the size of the crystals, and the whole of this should be crushed. and quartered down for the final sample. Unless this is done, it is manifest that the analysis may represent anything but the true average composition of the rock.

\section{B. CRUSHING.}

For accurate analyses the use of steel power crushers and mortars is out of the question because of the danger of contamination by particles of metal and the impossibility of cleansing the roughened surfaces after they have been in use a short time. Extraction, by the 
aid of a magnet, of steel particles thus introduced into the powder is quite inadmissible, since the rocks themselves, almost without exception, contain magnetic minerals.

a. SURVEY METHODS.

For breaking large pieces of rock to small sizes a thick iron plate with specially hardened surface and a similarly hardened pounder, such as street pavers use, will probably render the.best service, but the hardening must be done with extreme care.

A method of rough crushing on a small scale that gives fair satisfaction in practice is to place each fragment as received on a hard

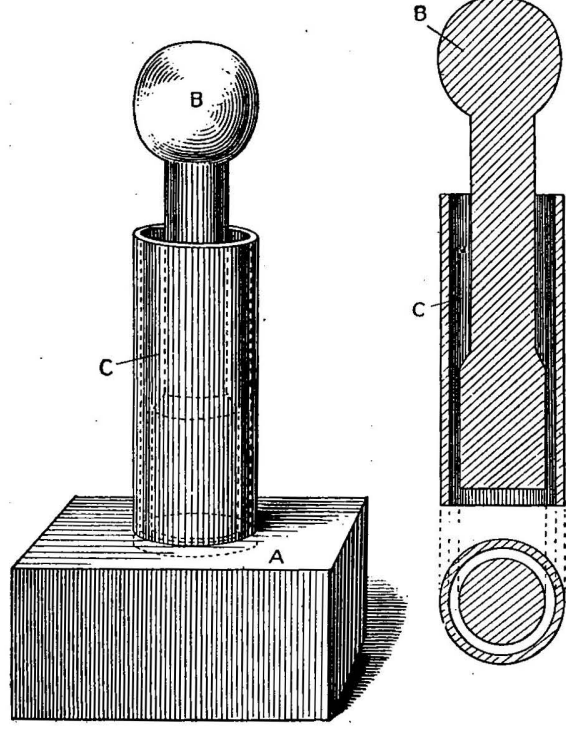

Fig. 8.-Ellis's mortar for crushing coarse materials. Ail parts of best chilled and surface-hardened tool steel. $\Lambda$, Block, $12 \frac{1}{2}$ by $12 \frac{1}{2}$ by $6 \mathrm{~cm}$., with depression in center $0.6 \mathrm{~cm}$. deep; $B$, pestle, $20 \mathrm{~cm}$. high, $3.5 \mathrm{~cm}$. diameter at base; C, cylinder, $12 \frac{1}{2} \mathrm{~cm}$. high, $5 \mathrm{~cm}$. outside diameter, $1.4 \mathrm{~cm}$. inside diameter, fitting accurately the depression in the block. steel plate about $4 \frac{1}{2} \mathrm{~cm}$. thick and $10 \mathrm{~cm}$. square, on which is likewise placed a steel ring 2 $\mathrm{cm}$. high and of about $6 \mathrm{~cm}$. inner diameter, to prevent undue flying of fragments when broken by a hardened hammer. In this way a considerable sample can soon be sufficiently reduced for transfer to the agate grinding mortar with a minimum of metallic contamination.

This method is row superseded in the Survey laboratory by one devised by C. W.' H. Ellis, mechanician in the physical laboratory of the Carregie Institution, which entirely obviates loss of material by flying fragments. The method of operating is apparent from fig. 8. The block, cylinder, and pestle are of the best chilled tool steel, surface hardened to the utmost linit.

The cylinder fits snugly into a depression in the block, after the manner of an ordinary "diamond" mortar, but the pestle has a diameter less than the inner diameter of the cylinder. The crushing is done by the pestle, without the aid of a hammer.

An imperative precaution, when using either of these methods, however, is to refrain absolutely from the least grinding or rubbing motion, with the hammer in the one case or the pestle in the other. Even quartz can be broken down by vertical blows to a size fit for the agate mortar without showing any darkening in color, whereas if a rubbing motion has been employed to even a limited extent, the 
powder will, by contrast, be perceptibly dark after grinding in the mortar. The hardest steel is very susceptible to abrasion by minerals less hard than quartz. The crushing must be carried to such a degree of fineness that after transferring the grains to the mortar the grinding motion of its pestle will not occasion further loss of flying particles, since the portions lost will, in case of all but glassy or very fine-grained material, not have the same composition as those retained.

b. WASHINGTON'S METHOD.

The rock is first reduced, as by the methods in a, to lumps that will fit into a "diamond" steel mortar of Plattner's form, the bottom of the cavity being hemispherical and matched in shape by the end of the pestle. The lumps are crushed, one by one, in the mortar by a dozen blows of a half-pound hammer (billet of wood, Dittrich), and the contents of the mortar are emptied into a cylindrical glass box about $3.5 \mathrm{~cm}$. deep and $7.5 \mathrm{~cm}$. internal diameter, with walls about 2 mm. thick. When the whole sample, or a quartered portion if large, has been thus crushed a piece of best silk bolting cloth with about 25 meshes to the centimeter is stretched over the open side of the box and clamped fast by a brass ring $1 \mathrm{~cm}$. in height. The box is now inverted and the finest powder gently sifted onto a sheet of glazed paper. The material left on the cloth is recrushed in the mortar and resifted, these operations being repeated until all has passed the sieve. It will, of course, not do to reject any portion, for thereby the composition of the mass will be affected. The sample is now fine enough to be reduced by grinding in a large agate mortar.

Doctor Washington finds that in this way there is practically no danger of contamination by metallic iron, and that any error resulting from introduction of cloth fiber is negligible. This last will hardly be true of the method practiced in parts of Europe (the German "beuteln"), which differs in essential points from the gentle sifting of Doctor Washington.

\section{GRINDING.}

Ordinarily an extremely fine state of division is unnecessary, except in the case of those portions in which alkalies and ferrous iron are to be determined, or where soluble constituents are to be removed by acids, etc., and in such cases the final grinding can be done at the balance table on a small portion slightly in excess of the quantity to be weighed off.

Formerly all rock samples in the Survey laboratory were, after crushing, reduced to powder by hand grinding, with expenditure of much time and labor. Since then, except in special cases, the work has been done by mechanical grinders run by a small electric motor. The grinder manufactured by the McKenna Brothers Brass Com- 
pany, Limited, of Pittsburg, Pa., is shown in fig. 9, with the addition of simple attachments intended to keep metal dust and oil out of the mortar. These additions have been found to be quite essential for our work. As made for the technical industries the machine is

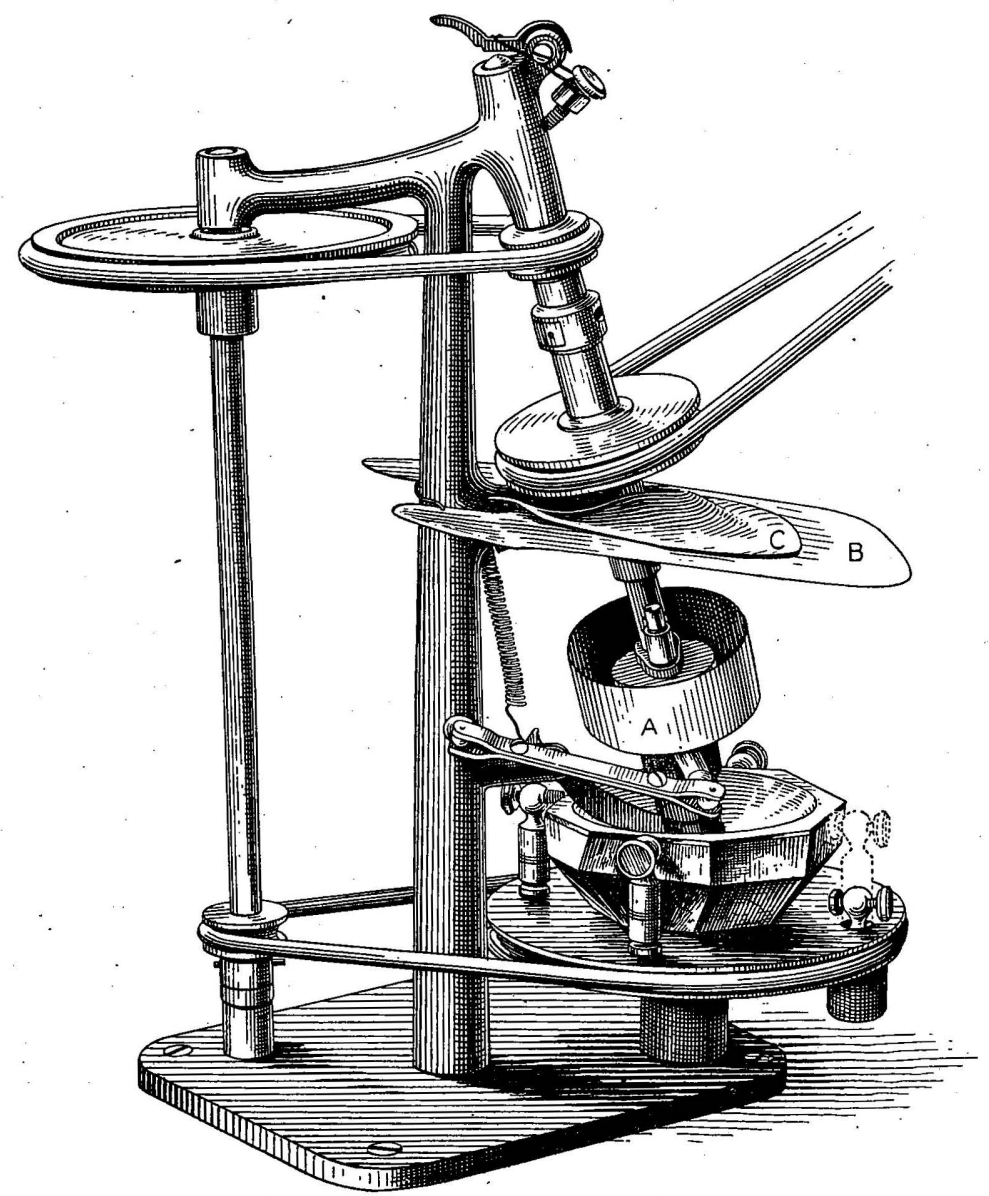

Fic. 9.-McKenna ore grinder, slightly modified by the addition of the following attachments to prevent oil and dust from belts and bearings fronı entering the mortar. $\Lambda$, Copper cup soldered to head of pestle holder. This partakes, without the slightest friction; of the motion of the latter. B, Tin plate with deep sinus, the latter being covered in turn by the rubber cloth, C, which fite closely about the metal shaft. "The spring at the tsp of the sMding rod, to which the agate pestle is attached at the bottom, can he adjusted to give any desired pressure or can be thrown back entirely to allow the pestlc to be raised in removing the agate mortar. This is accomplished by loosening a set screw and dropping one of the four posts holding the mortar in place. The pestle should make about 200 revolutions per minute, the mortar moving very slowly in the same direction. The scraper keeps the ore in the center of the mortar and the combined rolling and sliding motion controlled by the ball and socket supporting side arm reduces tho hardest ore very rapidly."-Iron A ge.

largely of brass, nickel plated, but the latest made for the Survey is of steel throughout, without plating. It was found that the pulley belts caused too much abrasion of the softer metal to permit its use for much of our work, on account of the danger of copper, zinc, and 
nickel getting into the sample in traces, and that in spite of a very limited use of oil this invariably became scattered over the contents of the mortar, though its presence never became visible to the eye except when white materials were ground. The last contamination is prevented by the copper cylinder, A, soldered securely onto the pestle holder so as to form a cup large enough for the purpose intended. The tin shield, B, with a superposed rubber cloth, C, covering the slit required for the proper adjustment of the shield, greatly lessens the danger of metallic contamination, even with the all-steel machine. At first it was thought that a rubber cloth covering the mortar, with the pestle snugly fitting a hole in the cloth, would be an effective protection, but it was found that there was sufficient friction on the rubber itself to cause contamination of the powder beneath.

With this apparatus a large amount of work can be done in a short space of time, though there is much loss of fine powder if the speed of revolution is too high. In our actual practice, three of these machines are operated, one or all, on a single table from the same shaft, the whole being covered with a glass case, divided by glass partitions, so as to exclude dust and separate the grinders.

The abrasion of pestle and mortar becomes far more manifest with these machines than with hand grinding, notwithstanding that each has its motion simulating as far as possible that of the hands. There is undoubtedly a very considerable introduction of silica into the sample from these sources, but in comparison with the amount invariably present in an igneous rock the quantity abraded is hardly of moment. Rocks normally free from silica, or nearly so, like many iron ores, are as a rule softer than igneous rocks and consequently cause less abrasion of the grinding implements.

W. Hempel ${ }^{a}$ has experimentally tested the effect of grinding in mortars of agate, glass, iron, and hardened steel, and has found that for hard bodies, like glass, hardened steel is far superior to agate, and that even mortars and pestles of green bottle glass showed much less abrasion than agate. In grinding 10 grams of glass to very fine powder the agate mortar and pestle, weighing 416 grams, lost 0.052 gram in weight (or 5 milligrams per gram of glass), against a tenth of that loss with a hardened steel mortar and pestle of the same weight that had been some time in use.

Notwithstanding these observations in favor of steel, the use of metal in rock analysis is, as said above, forbidden by the conditions of the case. Still more so is that of glass because of its complex composition. If impurity can not be avoided it is far better that it should be of one kind only, and that one productive of less disturbance than any other.

Metal sieves should never be used.

Bull. $305-07-4$ 


\section{WEIGHT OF GROUND SAMPLE.}

The sample when ground should weigh not less than 10 grams, and preferably 20 in case is should be necessary to repeat or advisable to employ unusually large portions for certain determinations, notably carbonic acid. Rock analysis has in this respect an advantage over mineral analysis, since material is almost always available in ample quantity and any desired number of separate portions may be used, whereas with a mineral the analyst is frequently compelled to determine many or all constituents in a single, often very small, portion of the powder. This course often involves delay and the employment of more complicated methods of separation than are usually necessary in rock analysis.

4. WATER-HYGROSCOPIC, ZEOLITIC, CRYSTAL.

A. GENERAL CONSIDERATIONS.

a. IMPORTANCE OF EMPLOYING AIR-DRY POWDER FOR ANALYSTS.

The time-honored custom of drying a powdered specimen before bottling and weighing has long seemed to me one that has no sound basis in reason. Its object is of course plain, namely, that of securing a uniform hygroscopic condition as a basis for convenient comparison of analytical results, since some rocks contain more hygroscopic moisture than others. Nothing, however, is more certain than that by the time the substance is weighed it has reabsorbed a certain amount of moisture, small indeed in most cases, but very appreciable in others; and further, with every opening of the tube moisture-laden air enters and is inclosed with the remainder of the dry powder. It therefore may very well happen that a powder at first dry will, after several openings of the tube, especially at considerable intervals, be nearly as moist as when first inclosed. Doctor Dittrich has expressed views similar to the above.

It is preferable to weigh the air-dry powder and to make a special. determination of moisture. If all the portions necessary for an analysis are weighed out one after another, or even at different times on the same day, the error due to difference of hygroscopicity in dry and moist weather, which for most of the separate portions is an entirely negligible quantity; is eliminated. Only in the main portion, in which silica and the majority of the bases are to be estimated, can it ever be an appreciable factor.

$$
\text { b. TEMPERATURE OF DRYING. }
$$

As to the temperature to be adopted for drying in order to determine so-called hygroscopic moisture, the practice has varied at different times and with different workers, ranging from $100^{\circ}$ to $110^{\circ}$. For the great majority of rock specimens it is quite immaterial which of 
these temperatures is adopted, since no greater loss is experienced at the higher than at the lower temperature, given a sufficient time for the latter. It is the present practice in this laboratory to employ a toluene bath giving a temperature of about $105^{\circ}$. Should the results show a very unusually high loss, the powder is reheated at, say, $125^{\circ}$, in order to learn if the loss is progressive with increased temperature. In the affirmative case it may be well to repeat the drying at $100^{\circ}$, for a. portion of the loss at $105^{\circ}$ was probably due to combined water from a mineral or minerals in the rock; but in that case even the loss at $100^{\circ}$ may sometimes very well include combined water, in which case drying over sulphuric acid alone may be desirable, or over dry sand.

c. INDIRECT METHODS.

In the latter connection it is proper to point out certain pitfalls in the path of the unwary, which, however, are in part more likely to be encountered in the analysis of minerals, where their influence may be of far-reaching consequence.

A mineral which loses a great deal of water over sulphuric acid-2 or 3 per cent, for instance--may need an exposure of several days or even weeks for its complete extraction. If the weighings are made from day to day, the apparent limit may be reached long before all water really removable has been taken up by the acid. Whenever the crucible, after weighing, is replaced in the desiccator it is no longer in a dry but a more or less moist atmosphere, and its contents, even when covered, sometimes absorb a part of this moisture and retain it so persistently that the acid is unable to bring the powder beyond its previous state of dryness in the next twenty-four hours. In fact, it may be unable even to reach it unless greater time is allowed. An experiment on 1 gram of tyrolite, made and published some years ago, seems to illustrate this point in part:

Experiment of drying 1 gram of tyrolite.

\begin{tabular}{|c|c|c|c|}
\hline $\begin{array}{c}\text { Time } \\
\text { exposed. }\end{array}$ & Loss. & $\begin{array}{c}\text { Time } \\
\text { exposed. }\end{array}$ & Loss. \\
\hline $\begin{array}{r}\text { Hours. } \\
18 \\
26 \\
23 \\
24\end{array}$ & $\begin{array}{c}\text { Gram. } \\
0.0231 \\
.0083 \\
.0029 \\
.0012\end{array}$ & $\begin{array}{r}\text { Hours. } \\
24 \\
24 \\
48 \\
24\end{array}$ & $\begin{array}{l}\text { Gram. } \\
0.0002 \\
.0003 \\
.0006 \\
.0002\end{array}$ \\
\hline $\begin{array}{r}24 \\
\quad 25 \\
\end{array}$ & $\begin{array}{l}.0001 \\
.0003\end{array}$ & 283 & .0380 \\
\hline
\end{tabular}

The experiment might reasonably have been considered ended after the one hundred and fifty-eighth hour, when a loss of but 0.1 milligram was shown during twenty-four hours; but nevertheless a nearly steady loss of 0.3 milligram per day took place for six days more, and might have been longer observed but for the interruption of the experiment. 
Again, it is a common practice to determine the water given off by hydrous minerals in an air bath at temperatures at and above $100^{\circ}$. To insure accuracy this experiment should never be made in crucibles or dishes which must be cooled in a desiccator. One instance will suffice: A gram of a mineral mixture containing about 17 per cent of water, of which about 3 per cent was driven off at $100^{\circ}$ and 8 or 9 per cent at $280^{\circ}$, was, after several hours' heating at the latter temperature placed in a desiccator over sulphuric acid and weighed as soon as cold, then replaced and again weighed the next day. It had regained $1 \frac{1}{2}$ per cent of its original weight, although the desiccator was tightly closed and the crucible covered, showing apparently a drying power superior to that of the acid.

A specimen of tyrolite was found on one occasion to lose 10.34 per cent at $280^{\circ}$, and on another occasion 14.33 per cent. In the latter case the drying and heating at progressive temperatures had continued during a period of 528 hours, the weighings being made usually from day to day; whereas in the former the duration of the experiment was much shorter and the intervals between weighings were but a few hours each.

An important research of C. Friedel ${ }^{a}$ well shows what errors are possible in the determination of this easily removable water, since he found that certain zeolites which had been largely dehydrated but not heated to the point of rupture of the molecular net could then absorb, instead of water, various dry gases in which they might be placed, as carbon dioxide, ammonia, carbon disulphide, and others, even air in large quantities, and certain liquids. In the light of this observation the cause of the great increase of $1 \frac{1}{2}$ per cent in weight of the partially dehydrated mineral mentioned above may very possibly be attributed to air from the desiccator instead of moisture, as was at the time supposed. At any rate, as Friedel says, the danger of accepting a loss in weight as an index of the amount of water lost is clearly shown, and thus that method of determining water is for many cases fully discredited. Just what method to adopt must be largely left to the judgment of the operator, who will often be guided by the mineral composition of the rock as revealed by the unaided eye or the microscope.

Friedel ${ }^{b}$ indicates a means for determining the true weight of water lost by minerals behaving like the zeolites, even without collecting the water lost, namely, by driving out of the dehydrated and weighed mineral, under proper precautions, any air it may have absorbed in the process of drying and cooling, and collecting and measuring this air and thus finding its weight, which, added to the apparent loss, gives the true contents in water. 
I have shown ${ }^{a}$ the importance of employing in desiccators only sulphuric acid of maximum dehydrating power. Acid that has stood long in desiccators that are in constant use is by no means so efficient as fresh, strong acid.

But aside from the above reasons for avoiding the use of indirect methods of water determination, there is another very important one. They never give correct results where air baths are used in the ordinary way, for the reason that the drying is performed in an atmosphere far from dry, and the substance can not, therefore, lose all its moisture. As examples, if the indirect method shows 0.12 and 0.75 per cent in two samples, the direct method may afford 0.17 and 1.00 per cent respectively. Similar observations have been repeated many times, and admit of no dispute. They apply as well to rocks as to minerals. Therefore, if the moisture content is more than an insignificant factor the indirect method must be avoided, or so arranged as to insure a constantly changing dry atmosphere.

To effect this the powder should be heated in a weighed tube, through which a current of dry air can be passed, and allowed to cool therein, after which a second weighing gives the loss of moisture. The degree of drying will depend on the nature of the reagent used for drying the air, sulphuric acid being more effective than calcium chloride, and phosphoric pentoxide most effective of all. When using the last, it is well to cause the air first to pass over or through one of the first named dehydrants, for its effectiveness rapidly lessens as it becomes glazed with metaphosphoric acid. For experiments carried out at room temperatures no bath is required, but for artificial temperatures the tube should pass through a suitable thermostat

\section{d. DIRECT METHOD.}

In general it is preferable to collect and weigh the moisture in suitable absorption tubes after its expulsion by the aid of apparatus described in $\mathrm{B}$, page 56 , even though the long time often required is sometimes an objection, since the absorption tube may gain weight, other than that of water from the mineral, sufficient to introduce an appreciable error. It may in such cases be necessary to run a long blank test for the correction to be applied.

e. ARGUMENT IN FAVOR OF INCLUDING HYGROSCOPIC WATER IN SUMMATION.

The question has been asked: "If the so-called hygroscopic water is not always such, but not infrequently includes combined water, why is not its determination and separate entry in the analysis entirely unnecessary? Why make a distinction, which, after all, may not be 
a true one?" The question involves the further consideration of the advisability of including in the analysis at all the loss at $100^{\circ}$ or $110^{\circ}$.

It would seem to be a reasonable desire on the part of petrographers to have all analyses referred to a moisture-free basis in order that they shall be strictly comparable. This involves the omission of the "hygroscopic" water from the list of constituents, which would be eminently proper were it always possible to be sure that the loss at $100^{\circ}$ to $110^{\circ}$ truly represents mechanically held water. Since it very often represents more; and the determination as to whether or not it does in each case is not always possible and would add to the time required for the analysis, it seems necessary to include this water. What errors may arise from its exclusion the following rather extreme case well illustrates: Certain rocks of Wyoming in powder form lost from 1 to 2 per cent of moisture at $110^{\circ}$. That not even an appreciable fraction of this was truly hygroscopic is fully demonstrated by the fact that the uncrushed rocks lost the same amount; yet the rule followed by many chemists and petrographers would have involved the removal of all this water as a preliminary to beginning the analysis, and not only would a most important characteristic have passed unnoticed, but the analyst would have reported an incorrect analysis, inviting to false conclusions and possibly serious confusion. (See also second paragraph of footnote, p. 39.)

f. SEPARATE ENTRY OF HYGROSCOPIC AND COMBINED WATER.

To revert now to the primary question, it may be said that the estimation of the loss at $100^{\circ}$ or $110^{\circ}$ and its separate entry in the analysis is advisable as not infrequently affording at once to the lithologist an indication of the mineral character of one or more of the rock constituents, thus perhaps confirming the microscopical evidence or suggesting further examination in that line. An unusually high loss at $100^{\circ}$ would be regarded as probable evidence of the presence of zeolites or other minerals carrying loosely combined water. It has been objected that the true hygroscopic moisture varies with the degree of comminution of the sample and with the condition of the air at the time of weighing, and that it is therefore improper to incorporate it in the analysis; but this variation is ordinarily not at all great. Perhaps the time may come when it will be the rule to ascertain by additional heating at a higher temperature whether the water lost at $100^{\circ}$ is to be regarded as purely hygroscopic. In such case it would be proper to omit it, and a distinct advance would undoubtedly be scored.

g. IS ALL TRUE HYGROSCOPIC WATER EXPELLED AT $100^{\circ}$ ?

It has been tacitly assumed in the foregoing that true hygroscopic water can all be expelled at $100^{\circ}$, which perhaps is not to be accepted as universally true. Eminent authority holds that it is impossible, 
in the cases of certain foliaceous minerals, notably the micas, to thus entirely remove it, and that a part is driven off only at higher temperatures. If this is true, a further uncertainty is introduced in its determination, which not only strengthens the argument in favor of entering all water in the tabulation, but also serves to emphasize the difficulties of the situation.

The following excerpt from the work of Doctors Day and Allen ${ }^{a}$ on the feldspars, already cited, has direct and important bearing on the above.

There is another error to which accurate specific-gravity determinations upon powdered minerals will be subject unless suitable precaution is taken. The exposure to the air during the period of grinding the samples gives opportunity for the condensation of sufficient atmospheric moisture upon the grains to affect the weight in air. The amount varies measurably with the size of the grains, as will be seen from the accompanying data, and probably with the degree of saturation of the atmosphere and the time of exposure.

Determination of moisture in 1 gram of powdered mineral upon exposure to the air.

$[<=$ finer than. $>=$ coarser than.]

\begin{tabular}{|c|c|c|}
\hline Mineral. & Fineness (mesh) & Moisture. \\
\hline 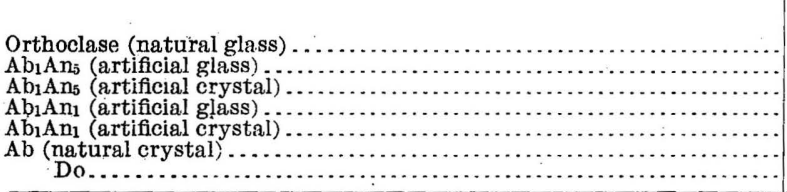 & 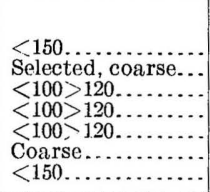 & $\begin{array}{r}\text { Gram. } \\
0.0061 \\
.0000 \\
.0010 \\
.0007 \\
.0010 \\
.0006 \\
.0069\end{array}$ \\
\hline 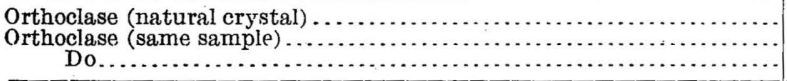 & $\begin{array}{l}<120>150 \ldots \ldots \ldots \\
<150 \ldots \ldots \ldots \ldots \\
\text { Still finer........... }\end{array}$ & $\begin{array}{l}.0011 \\
.0031 \\
.0059\end{array}$ \\
\hline $\begin{array}{l}\text { rthoclase (artificial glass) } \ldots \ldots \ldots \ldots \\
\text { rthoclase (portion of same) } \ldots \ldots \ldots \ldots\end{array}$ & $\begin{array}{l}\text { Everything }<100 \\
>150\end{array}$ & $\begin{array}{l}.0065 \\
.0022\end{array}$ \\
\hline
\end{tabular}

In the last two groups, note that the moisture in graded portions of the same sample varies with the fineness.

We also verified the conclusion of Bunsen $b$ that this absorbed moisture is not altogether removed at temperatures only slightly above $100^{\circ}$, but requires $600^{\circ}$ to $800^{\circ}$ - equivalent to low red heat. Several samples for which the moisture had been determined were laid away in corked test tubes for a number of weeks, after which redetermination gave exactly the former value.

It is worth noting in this connection that these measured quantities of absorbed water are of the same order of magnitude as those usually obtained for the water content in feldspar analyses, where again, of course, the finer the sample is ground for the analysis the greater the possible error from this cause. It may be that a part and occasionally all of the moisture usually found in these analyses is adsorbed and the significance of its presence there mistaken.

\footnotetext{
a Pub. Carnegie Institution of Washington, No. 31, pp. 56-57; Am. Jour. Sci., 4th ser., vol.: 19, 1905, p. 93.

b Wied. Ann., vol. 24, 1885, p. 327.
} 
B. APPARATUS FOR THE DIRECT DETERMINATION OF WATER AT DIFFERENT TEMPERATURES.

GENERAL PRECAUTIONS.

A precaution too often overlooked is to see that the drying agent used for the air current is the same as that in the absorption tube in which the water from the sample is collected-not only the same in kind, but as near as may be in strength as well, especially with calcium chloride. Therefore, the contents of drying and absorption apparatus should be changed often, and when refilled the reagents should be taken from the same stock. Fresh calcium chloride in absorption tubes should be exposed to a current of carbon dioxido and the excess removed by air before use. Furthermore, all connections should be as nearly as possible glass against glass, with the least exposure of rubber. With long rubber connections the error may be a very sensible plus one. Again, between refillings of the absorption tube the direction of the air current through it should always be the same. Care must also be taken that the conditions at the several times of weighing of the tubes are nearly alike as to temperature and hygrometric state of the atmosphere, for the afternoon weight of a tube may be appreciably different from that on the following morning. Finally the electrifying effect of wiping a tube before placing it on the balance must not be overlooked. In my experience this has ofter ? amounted to as much as 1 to 2 centigrams (always an increase in weight) after wiping with a clean linen handkerchief. The charge can be removed by repeated application of the hand, but disappears slowly without such aid. A strongly electrified condition makes itself apparent by marked irregularities in the swing of the needle. While more pronounced in cold weather, the phenomenon is not peculiar to winter.

a. STEIGER'S APPLICATION OF THE TOLUENE BATH.

The apparatus devised by George Steiger consists simply of a U-tube of home construction and a small toluene bath, in the cover of which is an opening of suitable shape to receive the tube (fig. 10). The open space between the uprights is closed when in operation by a piece of asbestos board. The horizontal end of the tube is made long, so that in the event of much water being given off it can be readily driven over into the absorption tube by a low flame, which if applied from the first may prevent any appreciable condensation of water before reaching the calcium chloride. The interior of the tube having been first freed from adhering moisture by a current of dry air and the absorption tube attached, the stopper is lifted without shutting off the 
air current, and the weighed powder quickly introduced by means of the clean metal trough in which it has been weighed (fig. 4, p. 31). The stopper is as quickly replaced and the dehydration begins at once and is often nearly finished by the time the thermometer indicates the maximum temperature.

By employing a U-tube with glass stoppers, like A, fig. 11, it would be quite possible to ascertain the loss in weight of the powder as well as the gain of the absorption tube, or to do away with the latter altogether. In this case, however, it would be necessary to vaseline the stoppers very lightly, and perhaps to introduce the powder through a dry funnel because of the vaseline film on the inner side of the end of the tube. The moderate heat on the top of the bath would suffice to prevent condensation of moisture before reaching the absorption tube, or to soon remove what might condense at first, but there must be no long rubber connections.

By employing a suitable oven this form of tube can be used for higher temperatures, but the stoppers must fit air-

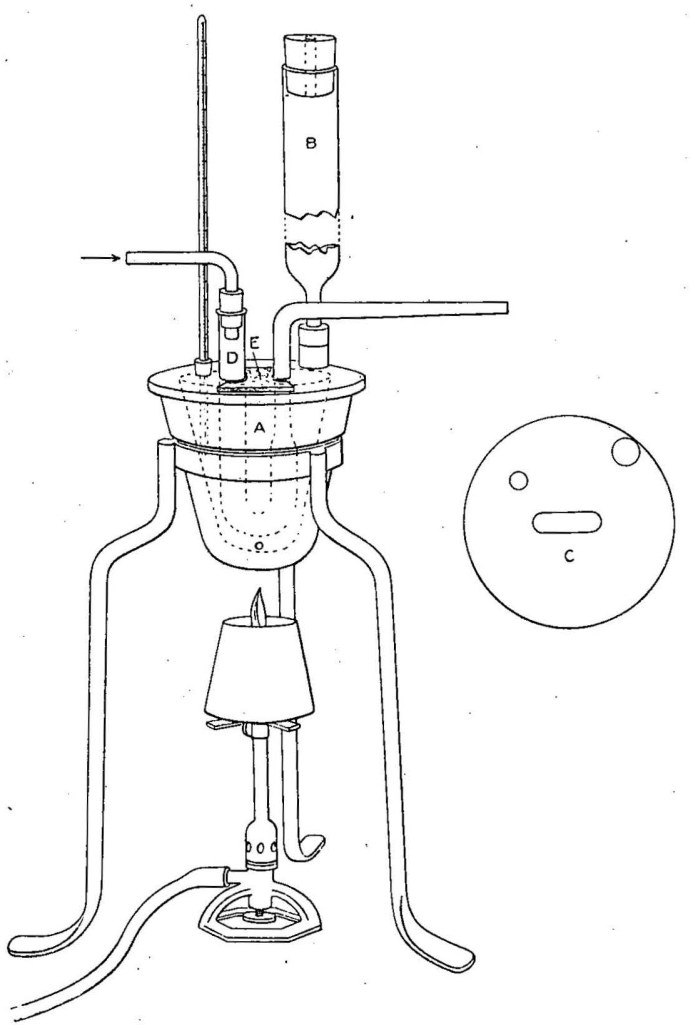

FIG. 10.-Steiger's form of drying apparatus. $\Lambda$, Toluene bath; $\mathrm{B}$, condencer, about $50 \mathrm{~cm}$. high; $\mathrm{C}$, cover of bath, showing openings; D, tube for mineral powder; F, asbestos board cut so as to close central opening in cover. tight, for vaseline can not be employed.

b. CIIATARD'S OVEN FOR TEMPERATURES UP TO $300^{\circ}$ AND OVER.

A form of drying oven devised by Dr. T. M. Chatard ${ }^{a}$ is in use in this laboratory for determining water at different temperatures up to $350^{\circ}$, and gives entire satisfaction. It is an asbestos-covered copper box, B, shown in different aspects and parts in fig. 11. The box is so constructed that the tube with its contents can be removed without detaching from either the drying or collecting tubes, which is a great advantage if it is desired to apply afterwards the direct heat

a Am. Chem. Jour., vol. 13, 1891, p. 110; Bull. U. S. Geol. Survey No. 78, 1891, p. 84. 
of a lamp in order to expel the water retained at $300^{\circ}$ to $350^{\circ}$. To facilitate this removal the stand is on rollers, so that after clamping the projecting end of the tube and removing the front of the box, $\mathrm{F}$, and the little side pieces, $\mathrm{S}$, closing the horizontal slits, the oven can be rolled bodily backward, leaving the tube and its attachments in their original position, ready for further heating over a burner or blast. The removable front, $\mathrm{F}$, of the oven is made of two pieces of sheet asbestos board stiffened by an interlaid piece of sheet copper. The inner piece of asbestos board fits snugly into the box, while the outer

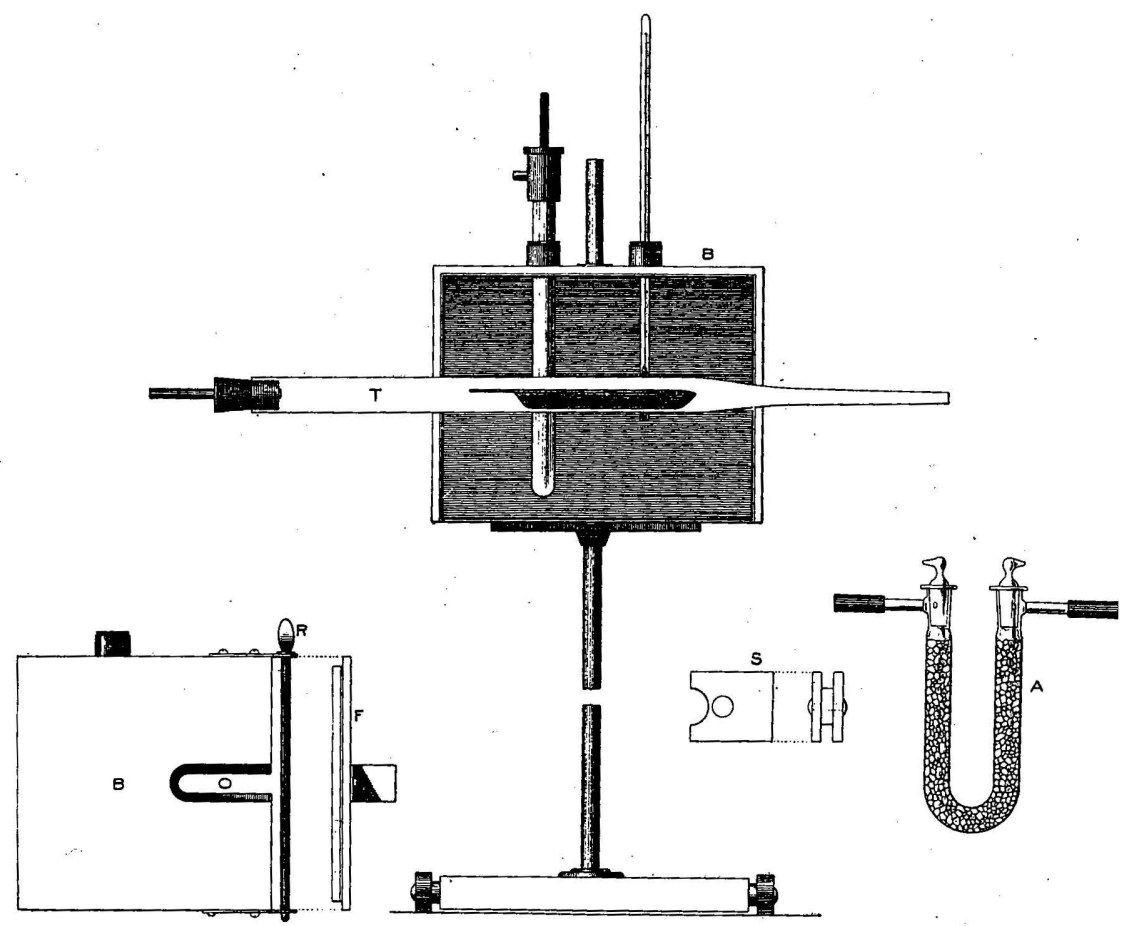

FIG. 11.-Chatard's form of drying oven for water determinations. B, Copper box, $18 \mathrm{~cm}$. long, $10 \frac{3}{2}$ $\mathrm{cm}$. high, $9 \mathrm{~cm}$. wide, open in front, its sides and top covered with asbestos board; $\mathrm{S}$, two slides of different sizes to close openings, $\mathrm{O}$, after the tube is in position; $\mathrm{F}$, asbestos-board front stiffened by an interlaid sheet of copper; $\mathrm{R}$, metal rod to hold front in place; T, glass ignition tube; $\mathrm{A}$, calcium-chloride absorption tube. For mineral substances with very high water content the tubes $T$ and A may preferably have the original form given them by Chatard, as depicted in Bull. No. 176, p. 37. The use of a straight tube, as shown in fig. 11, permits of a less complicated construction of box and stand than that here shown, since round holes in opposite ends of the box admit the igrition tube, and the roller base is then superfluous.

one, being slightly larger, by its projecting edges hinders the doc: from falling in and helps to prevent air currents. This door is held in place by the metal rod, $\mathrm{R}$. The little slides, $\mathrm{S}$, are made in a somewhat similar manner, and are intended to slip in from the front and close the two openings, $\mathrm{O}$, after the tube is in place, but before closing the front.

For other forms of tubes adapted to similar determinations, see pages 61 and 66-67. 


\section{WATER-TOTAL OR COMBINED.}

\section{A. ARGúments against "LOSS ON ignttion" Method.}

In a few cases the simple loss on ignition of a rock will give the total water with accuracy, but in the great majority there are so many possible sources of error that this old-time method can rarely be used with safety. Only when the rock is free from fluorine, chlorine, sulphur, carbon, carbon dioxide, and fixed oxidizable, or reducible $\left(\mathrm{MnO}_{2}\right)$ constituents can the loss be accepted as the true index of the amount of water present, and it is rare that a rock is met with fulfilling these conditions, especially as to the absence of ferrous iron. Blast ignition in presence of carbon dioxide alone of the above list may give a correct result, after separate estimation of the carbon dioxide, provided this emanates from carbonates of the earths and not from those of iron or manganese. The long-maintained idea that in presence of ferrous iron a sufficiently correct result is obtainable by adding to the observed loss an amount needed for oxidizing all ferrous iron is not justifiable. There can be no certainty that the oxidation has been complete, especially in the case of readily fusible rocks, and at the high temperature of the blast a partial reduction of higher oxides is not only possible but sometimes certain. The inability to insure complete oxidation by simple ignition is illustrated in the case of precipitated ferric hydroxide which has been ignited in contact with its filter paper. If the quantity was in any degree large it is sometimes decidedly magnetic, presumably from presence of magnetic oxide, which no amount of heating wholly oxidizes, especially in the larger grains. Neither is evaporation with nitric acid and reignition sufficient to destroy the magnetic property of the oxide, as has been claimed.

Direct weighing of the water evolved is then imperative in most cases, and of the numerous methods advocated, or in general use, several will now be considered.

B. DIRECT WEIGHING OF THE WATER WITHOUT THE USE OF ABSORPTION TUBES-PENFIELD'S METHODS.

a. FOR MINERALS EASILY DEPRIVED OF THEIR WATER.

If no other volatile constituents than water are present, the beautifully simple method first used by Prof. G. J. Brush and extended by Prof. S. L. Penfield ${ }^{a}$ leaves nothing to be desired for accuracy. It consists simply in heating the powder in a narrow tube of hard glass, enlarged at the closed end and provided with one or two further enlargements in the middle to hold the water and prevent its running 
back and cracking the hot glass. A capillary glass stopper fitted in with rubber tubing prevents loss of water by circulating air currents. The tube being held horizontally, the bulb is heated to any required degree by the Bunsen or blast flame. Moistened filter paper or cloth wound about the cooler parts of the tube insures condensation of all water. The heated end being finally pulled off, the tube is weighed after cooling and external cleansing, and again after the water has been removed by aspiration. For most rocks, as they contain little water, central enlargements of the tube are hardly needed.

Various forms of tubes used by Penfield are shown in fig. 12..

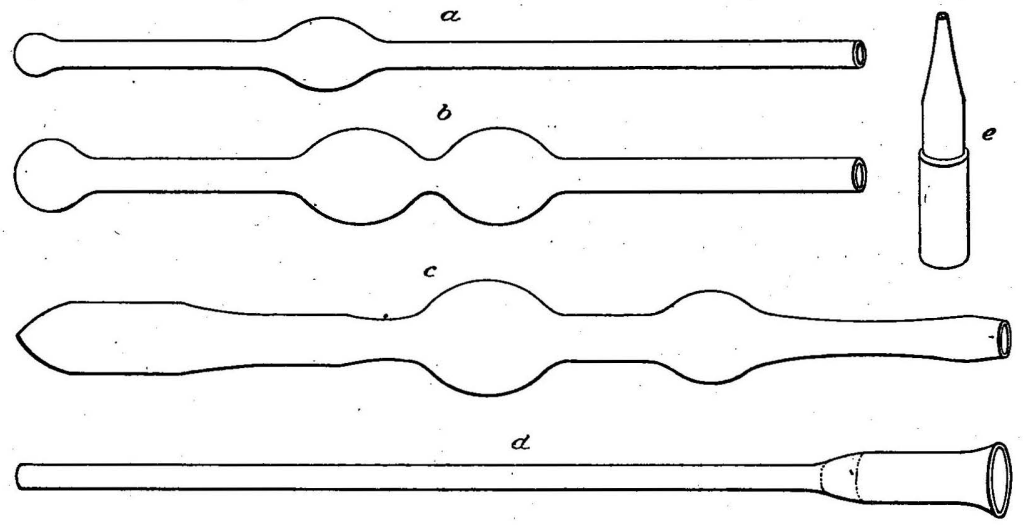

Fig. 12.-Penfield's tubes for water determination in minerals. $a, b, c$, different forms of tubes; $d$, thistle tube for introducing the powder; $e$, capillary-tipped stopper.

Before using, even if apparently dry, " these tubes must be thoroughly dried inside, which is best accomplished by heating and aspirating a current of air through them by means of a glass tube reaching to the bottom."

How this simple tube is made to afford entirely satisfactory results with minerals, even when carbonates are present, is fully set forth in the paper cited.

Few rocks, comparatively, are altogether free from other volatile constituents. Hence, for refined work the application of this apparatus in the simple manner above set forth is limited. It may, however, be used with the addition of a retainer for fluorine, sulphur, etc., in the shape of calcium, lead, or bismuth oxides.

b. FOR MINERALS NOT EASILY DEPRIVED OF THEIR WATER.

When minerals are present which do not give up their water wholly, even over the blast, as talc, topaz, chondrodite, staurolite, etc., Penfield's simple combination of fire-brick and charcoal oven, depicted in fig. 13, must be used, either with or without a retainer for fluorine, as circumstances demand. The part of the tube in the fire is to be protected by a cylinder of platinum foil tightly sprung about its end, 
and the part outside by asbestos board, as well as by wet cloth or paper. A piece of charcoal is likewise laid on the tube, as well as beneath and behind, and the blast flame is given a horizontal direction, so as to play upon the side of the apparatus. In this way a most intense temperature can be reached.

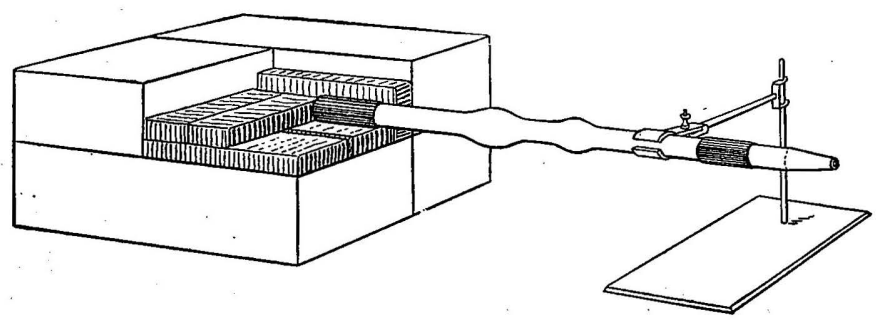

FIG. 13.-Penfield's fire-brick and charcoal oven for use in determining water.

In whichever way the apparatus may be used, the water found is the total water, from which that found separately at $105^{\circ}$ may be deducted if desired.

\section{c. DIRECT WEIGHING OF THE WATER IN ABSORPTION TUBES.}

a. PENFIELD'S PROCEDURE.

The simplest of these methods as to apparatus, and one permitting, by the use of auxiliary arrangements, such as are shown and described on page 58, the determination of the hygroscopic as well as any other fraction of the water, is the following glass-tube arrangement (fig. 14) of Penfield, ${ }^{a}$ whereby the brick and charcoal oven already referred to (fig. 13) comes again into play, but without the half brick shown in that figure.

The tube is of about $15 \mathrm{~mm}$. internal diameter, and is fitted with two platinum cylinders at $A$, ono inside, the other outside, where the heat exposure is to be most intense.

These are made from pieces of platinum foil, about $0.07 \mathrm{~mm}$. in thickness and 8 by 11 $\mathrm{cm}$. in diameter, which have been previously bent around glass tubes of such a size that when applied to the combustion tubing the spring of the metal will hold them in place.

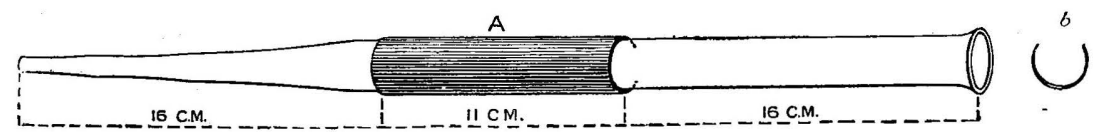

FIG. 14. Tube for water determination according to Penfield. $\Lambda$, Outer protecting covering of platinum foil. A second similar foil on the inside prevents the glass from collapsing when heated to softness. $b$, Cross section of platinum boat.

A large platinum boat, 7 to $8 \mathrm{~cm}$. long and 11 to $12 \mathrm{~mm}$. in diameter, with a cross section like $b$, should be used, since this will readily hold a gram of mineral mixed with 5 grams of sodium carbonate. $* * *$ The tube is placed in the angle formed by the charcoaI lining, some pieces of charcoal are placed at the sides in front, leaving an opening through which the flame may be directed, and an additional piece is laid on top. The tube can

a Am. Jour. Sci., 3d ser., vol. 48, 1894, p. 37, Zeitschr. anorg. Chemie, vol. 6, 1894, p. 22. 
readily be brought to a full white heat, and by forcing a slow current of dry air through the apparatus the carbon dioxide resulting from the decomposition can be removed and the water carried over into the weighed absorption tube. The glass fuses between the platinum casings, and in a number of experiments that have been tried there has not been a single instance where the glass tube has broken or shown any indication of breaking. After heating the tube will not crack if it is left to cool slowly on the charcoal, but it cin not be used a second time. *** At the high temperature to which the glass is subjected it of course becomes very soft and the ends must be properly supported; al:0 the rubber connections and absorption apparatus must be carefully screened by asbe:3tos board. By constructing a cover for the boat no material need be lost by spattering, and after making the water determination the contents may be used for the remainder of the analysis.

The inner cylinder of platinum serves to prevent the glass from collapsing as it softens, whereby distortion of the boat would result and its withdrawal for further examination of its contents would be impossible.

\section{b. GOOCH'S APPARATUS.}

Of more elaborate apparatus, designed to be used with fluxes, the tubulated platinum crucible invented by Doctor Gooch ${ }^{b}$ is capable of affording most excellent service, and it is the one by which far the larger number of water determinations in this laboratory have been and are made.

Fig. 15, which hardly needs detailed description, shows it in a modified form, which differs from the original forms of Gooch in that the tubes for connecting with both the drying and absorption vessels are constructed wholly of platinum instead of lead glass. The form of apparatus depicted in fig. 15 differs from that given in Bulletin No. 176 by a considerable shortening in length of the inlet and especially the outlet tube, whereby the cost is reduced and attachment can be made to a glass-stoppered $U$ tube, which is the most satisfactory form for absorption tubes. With tubes of the lengths shown in the figure there is absolutely no danger of their ends becoming hot enough by conduction to scorch or soften the rubber connection.

The extra first cost of the platinum extension to these tubes over the lead-glass ends of Gooch's original and modified forms need hardly enter as a factor into the question of employment of this apparatus. The glass ends often break, and only a rich lead glass, not easily obtainable, can be used, since it alone will not crack at the joint with the platinum after cooling.

As an adjunct to its convenient use there is needed an ordinary upright iron ring stand, with two small sliding rings, and a sliding ring burner provided with entering ducts for gas and air blast. Across the uppermost ring there is an arrangement of stout platinum wire ( $\mathrm{S}$, fig. 16), forming at the center of the ring a secure seat 
for the upturned flange of the crucible proper. Both rings and burner can be clamped firmly at any height.

The rock powder, having been placed in the cylindrical crucible (C, fig. 15), is there mixed with not more than 3 or 4 grams of fully dehydrated sodium carbonate, ${ }^{a}$ or more of lead chromate if carbon is to be likewise determined. The crucible is sunk in its seat, $\mathrm{S}$ (fig. 16), in the upper ring, $R^{\prime}$, and the tubulated cap, T (fig. 15), is fitted on and attached to the calcium-chloride drying towers-preceded by

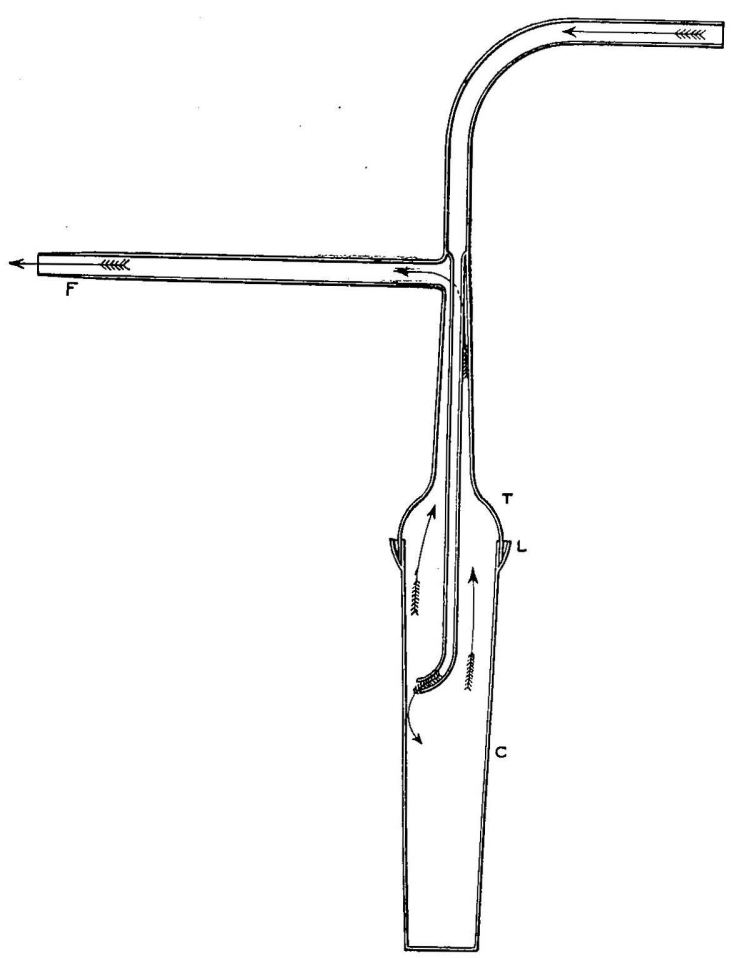

FIG.15.-Modified form of Gooch tubulated platinum crucible for the determination of water, onc-half natural size. Weight about 75 grams.

one containing potassium hydroxide if carbon dioxide is likewise to be estimated-on the one side, and to a sulphuric-acid bulb tube, B (fig. 17), on the other. Powdered sodium tungstate-free from arsenic, which would soon ruin the crucible lips-is now poured into the flanged lip, L (fig. 15), in which the cap rests, and a metal vessel of cold water having been raised up by the lower ring, $R^{\prime \prime}$ (figs. 16 and 17), until the platinum crucible is sufficiently immersed, the flame of

$a$ This has been heated for a length of time to near its fusing point over a free flame or in an air bath, to decompose the bicarbonate it uslally contains, and then placed in a destccator. Thus heated it is not very hygroscopic. Penfield found that 2.5 grams of it, spread out on a watch glass, gained only $0.0002 \mathrm{gram}$ in fifteen minutes. Potassium carbonate and potassium-sodium carbonate are too hygroscopic by far to be available. 
an ordinary blast lamp is turned on to melt the tungstate. As soon as this is fused the flame is removed and the salt solidifies and makes an air-tight joint, the test of which is the permanence of the column of sulphuric acid in the bulb tubes caused by the contraction of the air in the platinum apparatus as it cools.

After drying by a current of air at $105^{\circ}$ for two hours, more or less (see below, p. 66), by means of an air or toluene bath, as shown in fig. 16, the absorption tube, A (fig. 17), is interposed between the sulphuric-acid bulbs and the apparatus, and, while a slow current of

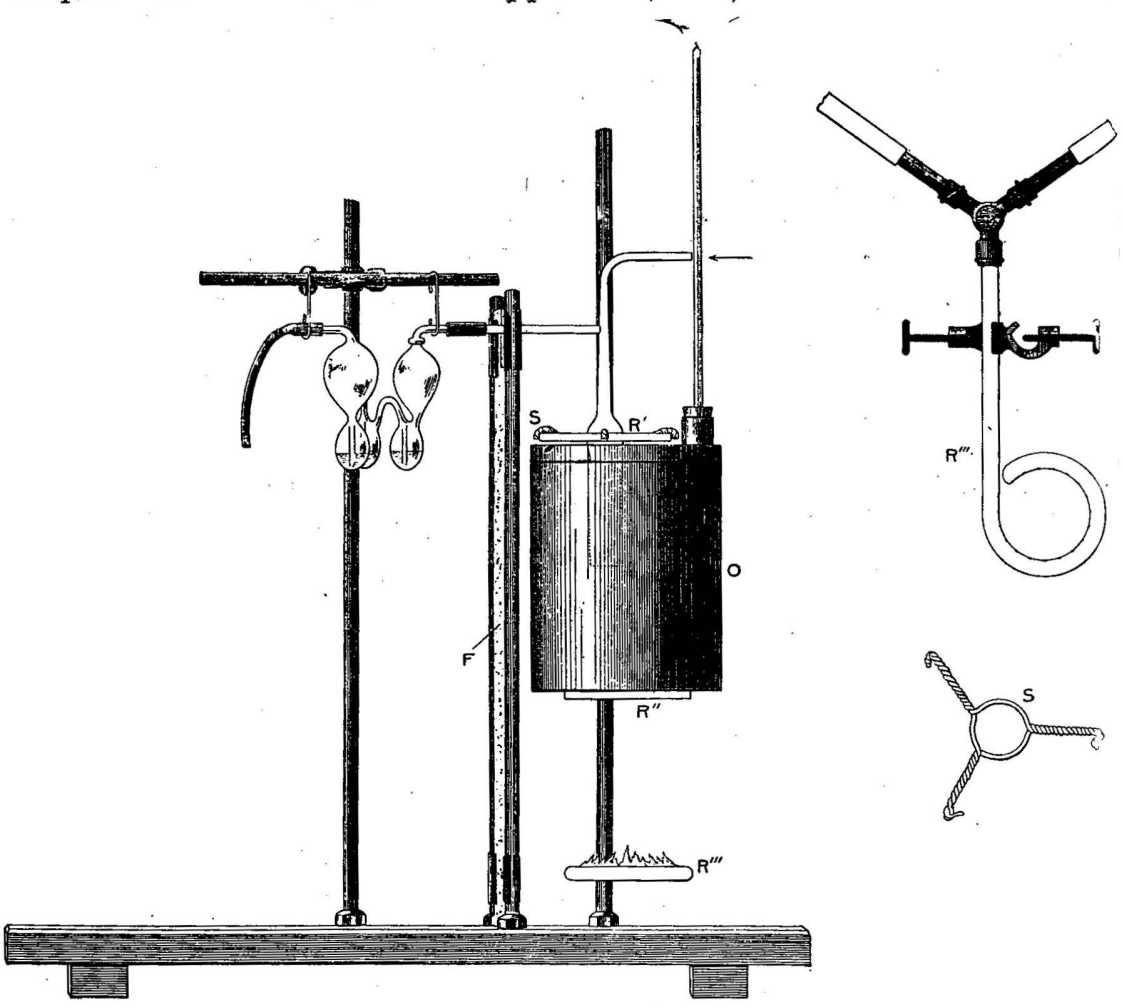

Fig. 16.-Arrangement, during drying, of Gooch apparatus for determining water. S, Seat of stout platinum wire resting on ring $R^{\prime}$ and serving as a support for the crucible; $R^{\prime \prime \prime}$, b!ast-fed ring burner; $\mathrm{R}^{\prime \prime}$, support for air or toluene bath, $\mathrm{O}$; $\mathrm{F}$, asbestos-board shield.

air continues to pass, the gradual heating and subsequent fusion of the flux is brought about by the blast-fed sliding ring burner, $\mathrm{R}^{\prime \prime \prime}$ (figs. 16 and 17). The sodium-tungstate joint is shielded from the flame by small pieces of asbestos board, P (fig. 17), cut out so as to fit the crucible. When fusion is complete, as shown in the case of sodium-carbonate flux by the decided slackening of the gas current through the safety bulbs attached to the drying tube, the flame is extinguished and a current of air is. allowed to eontinue until the apparatus is cold. 
This apparatus suffers from the drawback of being slightly permeable to combustion gases at high temperature. The defect can be overcome by causing the flame to play upon an outer ordinary platinum crucible, kept permanently filled with sodium-potassium carbonate. This protective crucible, however, is soon ruined for other purposes, being distorted by the alternate expansion and contraction of the carbonate.

It has been found that if the operation is carried out expeditiously and the final full heat applied for but a few minutes the error due to

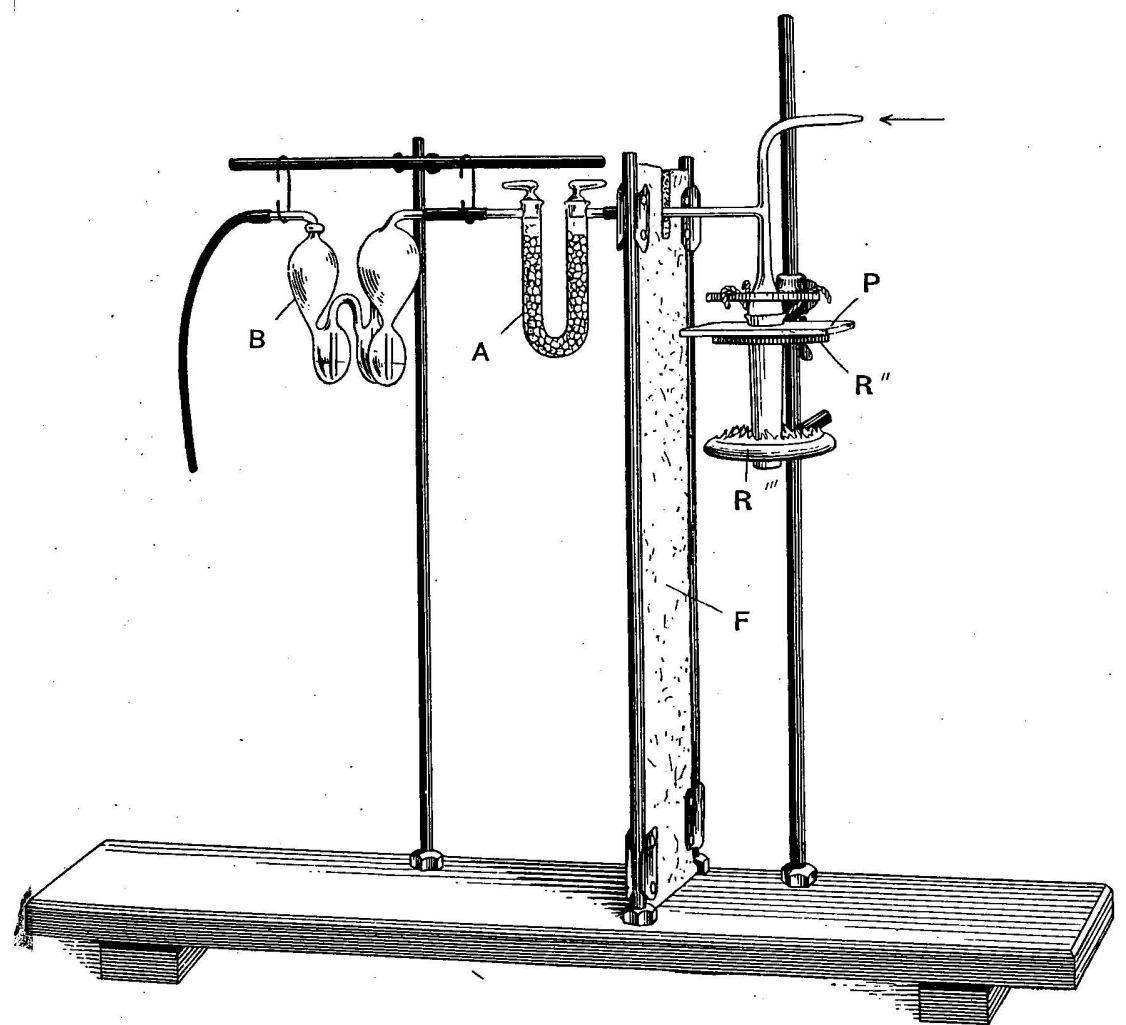

TIG. 17.-Arrangement, during fusion, of Gooch apparatus for determining water. $R^{\prime \prime \prime}$, Blast-fed ring burner; $P$, protective asbestos shield resting on ring $R^{\prime \prime} ; F$, asbestos-board shield; $A$, calciumchloride tube; $\mathrm{B}$, sulphuric-acid bulbs serving to show the rate of gas flow through the absorption tube and at the same time to prevent back entry of moisture from the air into A.

penetrating water gases is inappreciable. This hastening may be rendered safer by using rather finely powdered calcium chloride in the central section of the U-shaped absorption tube to avoid large air channels. Through this or any other apparatus based on similar principles the air current should always be forced, not drawn. A warm air blast directed upon the exit tube near its entrance into the absorption tube greatly shortens the time required and is to be recommended.

Bull. 305-07- 5 
In this apparatus only the water expelled above $100^{\circ}$ to $110^{\circ}$ should as a rule be determined, and to effect drying of the mixed mineral powder and sodium carbonate, after luting the tubulated eap on the cylindrical crucible with sodium tungstate, the tube is sunk through a round hole in the cover into a small cylindrical air bath (fig. 16), which can be heated from beneath by the same ring burner which is subsequently to fuse the flux. A slow current of air is then forced through and the drying satisfactorily accomplished.

The reason why it is unsafe to attempt estimation of "hygroscopic" moisture in this apparatus is that the luting of the two parts must be done by direct application of a flame to the tungstate, and considerable water vapor may enter the apparatus and be in part retained by the dried sodium carbonate.

c. MERITS OF THE ABOVE FORMS OF APPARATUS.

The apparatus described in $a$ and $b$ permit the determination of other constituents besides water in the same portion if necessary, and by the use of lead chromate instead of sodium carbonate, graphite, or the carbon of organic matter, can be simultaneously determined with the water.

To one accustomed to its use, and with a drying and suspension attachment permanently set up, the Gooch apparatus, considering its limitations above set forth, offers perhaps the most handy and convenient means for the determination of water in rocks. Its high first cost, in comparison with the glass tube, is fully made up in time by its durability.

d. JANNASCH'S METHODS.

This zealous deviser of methods for mineral analysis has published in the Zeitschrift für anorganische Chemie and the Berichte der Deutschen chemischen Gesellschaft several papers dealing with the problem of water determination in minerals, and in his text-book ${ }^{a}$. these are collected in more or less modified form.

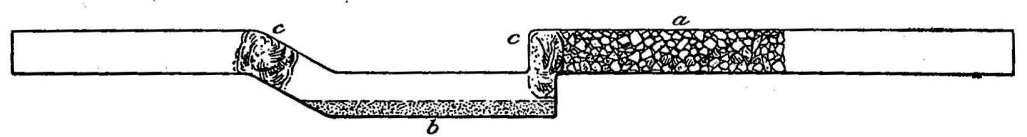

FIG. 18.-G lass tube for determination of water (Jannasch). $\quad b$, Mixture of mineral powder with borax; $c$, c, plugs of glass wool; $a$, layer of lead chromate or lead oxide. Total length of the tube, $33 \mathrm{~cm}$; inside diameter, $12-14 \mathrm{~mm}$.

For the majority of silicates he finds dehydrated borax powder a most efficacious flux, usually at a very moderate temperature. The fusion is accomplished either in a platinum boat within a glass tube or in a tube of the form and dimensions shown in fig. 18.

$a$ Praktischer Leitfaden der Gewichtsanalyse, Leipzig, von Veit \& Co. (1897). 
For rocks or minerals containing not much fluorine a retaining layer of granular lead chromate, or of previously fused and powdered lead oxide, is used as shown at $a$. Plugs of glass wool are used, at $c, c$. Whether or not the boat is employed, the borax is first introduced and, together with the retainer, is thoroughly dried out in an asbestos oven by a hot-air current. Then, after cooling, the mineral powder is added and thoroughly mixed with the borax. Heat is applied by a flat flame to the mixture, which soon melts and forms a clear fusion, when the action is complete. The blast may be used in extreme cases. The layer of retainer must be kept warm by an auxiliary flame, and the absorption tube must be removed before the flame under the fused mass is extinguished, for the glass breaks as soon as this is done. Carbon dioxide can simultaneously be determined by attaching a sodalime tube to the calcium-chloride tube. For one-half to 1 gram of silicate Jannasch uses $1 \frac{1}{2}$ to 2 grams of dehydrated borax.

Regarding the borax method, its inventor insists on the following points as essential to success, especially when the blast can not be applied: Most thorough mixing of flux and mineral powder and a most impalpable fineness of the latter:

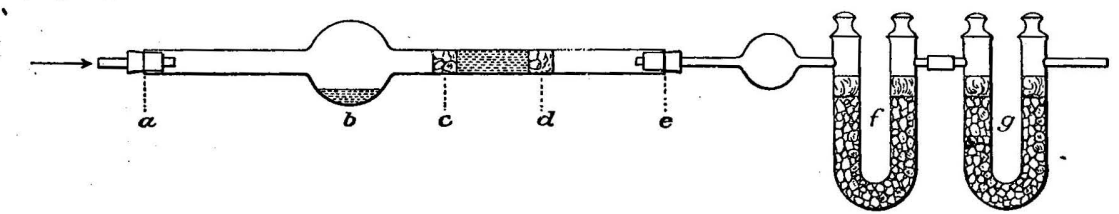

FIG. 19.-Glass tube for determination of water in special cases (Jannasch). Length from $a$ to $e, 26$ cm. ; inside diameter somewhat over $1 \mathrm{~cm}$; volume of bulb $b, 25 \mathrm{~cm} .{ }^{3} ; c d$, retaining layer of lead oxide between plugs of glass wool. $f$, calcium-chloride absorption tube; $g$, protective tube.

The borax itself is prepared by heating pure crystallized borax in a platinum dish till a small portion has melted. That remaining unfused is powdered and again heated in the dish to dull redness for fifteen minutes, with constant stirring. The powder is placed in a tube with tightly fitting glass stopper 'and kept over sulphuric acid. It must not be kept long without reheating, because of being hygroscopic.

Another form of tube used by Professor Jannasch for special purposes and recommended by Doctor Dittrich is shown in fig. 19. Minerals, such as topaz, which is not fully decomposed by the borax method and which contains a large amount of fluorine, are fused at $b$ with about six times their weight of lead oxide. A layer of lead oxide (equal parts of oxide and peroxide.-Dittrich) between $c$ and $d$ serves to retain any fluorine escaping from the fusion. Doctor Dittrich uses this form generally for substances which can not be heated in the simplest form of Penfield tubes and for those which give off their water on simple ignition, without admixture of lead oxide, though, of course, with the retainer for sulphur, fluorine, etc. 
If $\mathrm{CO}_{2}$ is to be simultaneously determined, any carbonate in the lead oxide must be first destroyed by heat. As in the preceding description the layer of retainer must be kept continually hot by an auxiliary flame.

6. SILICA, SEPARATION FROM ALUMINA, ETC.

A. alternative methods of DeComposing THE ROCK.

a. GENERAL CONSIDERATIONS.

The practice of separating alumina, etc., by the usual methods, after first attacking the rock powder by hydrofluoric and sulphuric acids-silica being estimated in a separate portion-while attractive in principle, was abandoned by me after fair trial, owing to the disturbance sometimes occasioned by incomplete expulsion of fluorine and to a less degree by the presence of sulphates instead of chlorides. With the exception of a comparatively few analyses made thus, the sodium-carbonate method has always been employed. In the case of rocks rich in fluorine strict accuracy would require the separation of silica to be made as in the Berzelius method for fluorine estimation (see p. 155, also footnote, p. 71), but in practice it is not often necessary to resort to this tedious procedure, since the amount of fluorine is usually small and it can by no possibility cause a loss of much more than three-fourths its own weight of silica by volatilization as silicon fluoride when the sodium-carbonate fusion is evaporated directly with hydrochloric acid. Probably the loss is less, since some fluorine perhaps escapes as hydrofluoric acid. However this may be, the error is of comparatively slight importance, since it always attaches to the constituent present in greatest amount.

Various fluxes other than alkali carbonates have been recommended for breaking up silicates insoluble in ordinary acids, such as lead and bismuth oxides, lead carbonate, borax, and boric oxide. Professor Jannasch and his pupils have been especially active in this line of work, as is evidenced by their numerous published papers. One of the advantages most of these fluxes possess over the alkali carbonates is their removability after serving their purpose, thus allowing the various separations to be made more perfectly and without the annoying interference of several grams of foreign fixed salts, which are most troublesome in that part of the analysis devoted to the separation of silica, alumina, iron, lime, and magnesia.

Another of their advantages is that with some of them it is possible to estimate in one portion the alkalies in addition to those constituents usually determined in the silica portion. Where the material is limited, as it so often is in mineral analysis, this is a most important 
advantage, sufficient to outweigh all possible objections; but in rock analysis, where the supply of material is usually ample, it is rarely worth considering. A still further point in their favor is that it is probably more easy to obtain them entirely free from fixed impurities than an alkali carbonate.

There are, however, objections to their use. With some of them an extraordinary amount of time must be devoted to grinding the mineral to an impalpable powder, and the flux itself may need considerable hand pulverization. Once introduced, they must be removed before the analysis can be proceeded with, and this removal takes much time and is always a possible source of error.

In mineral analysis these objections are entitled to far less weight than in rock analysis, since the object sought-usually the deduction of a formula-warrants the expenditure of much time and painstaking care. Finally, it has been found that one or more of these fluxes are not available for altogether general use, since certain minerals do not fully succumb to their attack under simple conditions, as andalusite with boric oxide and others with lead oxide (Jannasch). Therefore, however well adapted one or the other of these methods may be for the analysis of homogeneous minerals, it is very improbable that the anticipations of Professor Jannasch, to the effect that the boric-oxide method will soon supersede the alkali-carbonate fusion method in rock as well as in mineral analysis, will be speedily realized. Nevertheless, the boric-oxide fusion method, owing to its evident merit, will be described in detail after brief reference to a means of bringing refractory silicates into solution without employing any solid reagent.

The methods of decomposition and the subsequent treatment of the silica hereinafter described are with few exceptions applicable to definite silicate mineral species as well as to complex mixtures of them.

b. DECOMPOSITION OF REFRACTORY SILICATES BY HYDROCHLORIC ACID UNDER PRESSURE.

Jannasch $^{a}$ pours upon the finely ground rock powder contained in a platinum tube of about $26 \mathrm{~cm} .^{3}$ capacity a somewhat diluted hydrochloric acid ( 4 acid to 1 water), places over the open end a cap which does not hermetically close the tube, inserts the latter in a larger tube of potash glass likewise partially filled with the diluted acid, seals the glass tube, places it in turn in an inclined position in a steel Mannesmann tube containing ether or benzine to equalize the pressure, and heats to any desired temperature up to $400^{\circ}$.

The chief drawback seems to be a somewhat incomplete decomposition, doubtless due to the necessarily inclined position of the tube, which causes the powder to collect at the lower end, and thus renders decomposition less complete than if the material were spread evenly

a Ber. Deutsch. chem. Gesell., vol. 24, 1891, p. 273; Zeitschr. anorg. Chemie, vol. 6, 1894, p. 72. 
throughout the length of the tube. Further, the acid strongly attacks the platinum unless the air in both the platinum and the glass tubes is replaced by carbon dioxide. Even when this is done, several milligrams of platinum are found in the silicate solution.

Nevertheless, to those possessing the necessary platinum and steel tubes the method can render efficient service in special cases when economy of material is imperative.

c. THE BORIC-OXIDE METHOD OF JANNASCH AND HEIDENREICH. $a$

$\alpha$. Preparation of the boric oxide.-This demands, if the alkalies are to be estimated in the same portion as silica, etc., an absolutely alkali-free boric acid, which can be prepared by two or three recrystallizations of a good commercial article. The purified crystals are dehydrated and fused in a large platinum crucible. This is then suddenly cooled to cause the anhydride to crack into pieces of a size convenient for powdering, which are to be kept in a tight glass and powdered as needed, since the anhydrous oxide is hygroscopic.

$\beta$. Treatment of easily decomposable silicates. - To this flux Jannasch and Heidenreich find that nearly all silicates readily succumb over the ordinary blast lamp. The fusion is made in a large crucible holding $40-65 \mathrm{~cm} .^{3}$, and the proportion of flux to be used is gaged according to the nature of the silicate, ranging from 3 to 8 and more parts to 1 of mineral. This last must be finely powdered, especially the most resistant, the authors recommending the expenditure of one-half to one hour's time for the grinding of one-half to 1 gram of powder. A low burner heat is applied for five to ten minutes till water is expelled; the heat is then gradually increased till the gas is fully turned on. Bubbling and rising in the crucible is prevented as far as possible by using a short platinum rod which does not reach above the edge of the crucible. When the mass has been in quiet fusion for a time in the covered crucible the blast flame is applied. The average duration of the entire operation is twenty to thirty minutes, but depends much on the character of the mineral.

$\gamma$. Treatment of refractory silicates. - For those minerals which, like andalusite, cyanite, and topaz, are not fully decomposable by the heat of the ordinary blast flame, Jannasch and Weber ${ }^{b}$ use a flame fed by oxygen instead of air. The blast lamp, of $2 \frac{1}{2} \mathrm{~mm}$. opening, is supplied with gas from at least five or six ordinary gas cocks, and the flame is made broad and free from luminosity. The mineral having been first heated as above described, but with a much larger proportion of flux - as high as 30 to 1 - a few grams additional of boric oxide

\footnotetext{
a Zeitschr. anorg. Chemie, vol. 12, 1896, p. 208. This method of decomposing rocks with a view to the determination of their contained alkali originated with Sir Humphrey Davy, as shown in his paper "On a method of analyzing stones containing fixed alkali by means of the boracic acid" (Phil. Trans., 1805, p. 231; Ann. d. chim., vol. 60, 1806, p. 294; Gilbert's Annal., vol. 30, 1808, p. 369; Tulloch's Phil. Mag., 1806, p. 146). Original with Jannasch and Heidenreich is the manner of getting rid of the introduced boric oxide.

$b$ Ber. Deutsch. chem. Gesell., vol. 32, 1899, p. 1670.
} 
are added and the oxygen blast is applied till, in ten or fifteen minutes, the fusion is as transparent as glass. ${ }^{a}$

$\delta$. Further treatment after fusion.-From this point the further treatment is the same in both cases and, as modified by Jannasch and Weber ${ }^{b}$, is as follows:

The hot crucible is cooled in cold water and the contents are turned into a very large porcelain or platinum dish, to which, after covering with a glass, a saturated solution of hydrochloric-acid gas in methyl alcohol is added. ${ }^{c}$ The cover being then removed, the liquid is heated to boiling, over asbestos board, by an inch-high flame, with constant stirring, or it is left without attention over a lower flame or on a water bath heated short of boiling. The crucible is cleansed in a similar manner, and its contents are added to the dish. In ten to fifteen minutes, with occasional addition of the methyl chloride, solution is complete and the liquid is then boiled down to a small volume and evaporated to dryness on the bath. The residue is then digested on a bath at $80^{\circ}$ to $85^{\circ}$ three or four times in succession with the ether solution, in order to remove the last traces of boron as boric ether. Care should be taken to wash down from the sides of the dish, with methyl-chloride solution, the boric acid formed and deposited thereon during the evaporation.

$\varepsilon$. Possible objections to the boric-oxide method--Very much is claimed by Jannasch for this method, but with all its undoubted merit there arè two points which may militate against it. The boric ether, driven off in such quantities, at once decomposes in contact with moisture, and boric acid settles over all objects with which it comes in contact. The hood must become thickly coated. Hence a special hood for these evaporations alone seems to be called for, otherwise boric acid may at any time fall into other dishes and cause untold trouble. The second objection attaches to the use of the oxygen flame when alkalies are to be estimated in the fusion, and the ability to so determine them is one of Jannasch's chief claims in favor of the method, for it can not be doubted that at the high temperature of this flame alkalies are volatilized. Borax can be slowly but wholly volatilized over the ordinary blast, hence there is great reason to fear sufficient loss at this much higher temperature to give rise to serious error at times.

d. THE SODIUM-CARBONATE METHOD.

$\alpha$. Advantages of sodium carbonate over sodium-potassium carbonate.-Except in special cases, as when fluorine or chlorine are to be

\footnotetext{
a An interesting and important observation reported by Jannasch and Weber is that when the oxygen blast has been used for silicates carrying fluorine or mixed with fluorides the fluorine seems to be wholly expelled as boric fluoride without loss of silica. If this should prove to be generally true, an easy way is at last afforded for determining silica in such cases, where even its detection, when present in small amount, has heretofore been difficult.

$b$ Ber. Deutsch. chem. Gesell., vol. 32, 1899, p. 1670.

c Made by passing dry hydrochloric-acid gas into cooled methyl alcohol for from one to two hours.
} 
determined, there is no advantage in using the much recommended, because more fusible, double carbonate of sodium and potassium, or the equimolecular mixture of the normal carbonates, or of sodium carbonate and potassium bicarbonate. As Dittrich says, ${ }^{a}$ potassium salts are more prone to pass into precipitates than sodium salts and it may be that the higher melting point of sodium carbonate is a distinct advantage. Certainly, for effective decomposition of some rock constituents, a far higher temperature than that of the fusing point of the double salt is required.

B. Purity of the sodium carbonate.-Notwithstanding the most earnest efforts for years, it has been impossible to procure, either in the open market or by special arrangement with manufacturers, a grade of sodium carbonate which can be called chemically pure. With special precautions small lots can be prepared in the laboratory that will contain less than 1 milligram total impurity in 10 grams; but such an article can not be purchased in the market, and rarely will the so-called chemically pure dry sodium carbonate contain as little as 1 milligram in 10 grams. The invariable contaminating substances-aside from sand and straw, which have sometimes been found in large amount-are silica, alumina, iron, lime, magnesia, and sometimes phosphoric oxide, all of these going into aqueous solution with the carbonate. The chief of these impurities are usually silica, alumina, and lime. An article of the above degree of purity is satisfactory in almost all imaginable cases, since the use of the usually extravagant amount of 10 grams for a fusion would introduce an error of but 0.1 per cent in the analysis, supposing 1 gram of mineral to be operated on, and it would, moreover, be distributed over several constituents. This error is undoubtedly fully equaled by the introduction of dust from the air in the various long evaporations.

It is to be borne in mind that the so-called dry sodium carbonate, "C. P," almost always contains some bicarbonate and hence yields water on heating. This, however, in no way detracts from its usefulness, and it is not necessary to convert it wholly to the normal salt for fusions of the kind now contemplated. In fact, C. Holthof ${ }^{b}$ most strongly recommends using the bicarbonate itself as a flux instead of the normal carbonate (see $\delta$, p. 74).

$\gamma$. The fusion with normal sodium carbonate.-Ordinarily from 4 to 6 parts of the flux should be used to 1 of rock powder, thoroughly mixed in a crucible of 20 to 30 grams weight. It is inadvisable to use the much larger proportion of flux recommended by some writers, except as it may be shown in isolated instances to be needed. The larger amounts introduce more impurity than the smaller and necessitate longer washing of precipitates. At first the crucible (covered) is 
placed over a moderately low Bunsen flame, which is gradually increased to the maximum and maintained there till the mass is quiescent. There should be no violent action. The contents of the crucible will then appear, in the case of highly feldspathic or quartzose rocks, as a viscous liquid, occasionally almost clear, though generally more or less turbid, and when placed over the blast little or no further effervescence occurs. Melts of this character disintegrate very readily in water. With less siliceous rocks the fusion is less perfect and may be far from complete, though this does not necessarily imply incomplete decomposition of the silicates. It is generally advisable and often necessary to place these less fusible mixtures over the blast lamp, when a further and very marked escape of carbon dioxide takes place, and the crucible cover should be cautiously lifted at intervals to avoid loss by boiling over. This boiling is due not merely to further action of the carbonate on the rock-forming minerals, but oftentimes more to the decomposition of the alkaline-earth carbonates, whose bases then enter into combination with other constituents of the flux and rock to form compound silicates and probably aluminates. It is a great mistake to regard, as many seem to do, the alkaline-earth metals-magnesium, iron, and manganese-as present in the form of carbonates after a blast fusion. They are rarely in that state even when only the Bunsen flame has been used.

The blast flame during fusion should not be directed vertically against the bottom of the crucible, but at an angle against the side and bottom, nor should the flame be allowed to envelop the whole crucible. These precautions apply in all ignitions of reducible substances, and yet they are rarely observed. In neither case, if neglected, will there be the necessary oxidizing atmosphere within the crucible; on the contrary, reduction may occur fraught with serious consequences. This is especially true if the rock contains more than traces of pyrite or other sulphide, when, after cleansing and igniting the crucible, there may appear on its interior a darkening due to oxidation of reduced iron which had alloyed with the platinum. This may in exceptional cases amount to several milligrams in weight, and can be removed only by repeated ignitions, followed each time by scouring or treatment with hydrochloric acid or acid potassium sulphate. In order to avoid the use of niter in case of pyritiferous rocks, it is well to first roast gently the weighed powder in the crucible in which the fusion is to be made, turning the crucible around a few times to expose all of the powder to the air. With rocks exceptionally high in pyrite the roasting is best done in a porcelain crucible. In such case, after transferring the greater part to the platinum crucible, if brushing with a camel's-hair brush does not remove the last of any adhering dust, scouring with a little sodium carbonate will be effective.

It sometimes happens that the cooled flux, and even its solution, 
will indicate absence of manganese when it is really present in quantity to give normally a strong coloration. Two fusions made side by side or successively, under apparently similar conditions, may in one case show little or no manganese, in the other considerable. This observation has been frequently made, and therefore the absence of a bluish-green color in the fusion is not to be taken as proof of the absence of manganese. This difference of behavior I can ascribe to no other cause than that of a reducing atmosphere in one of the crucibles and an oxidizing one in the other, even though the conditions were apparently alike. It is, of course, not to be expected that the green color can show until all other oxidizable components of the rock, like sulphides, ferrous iron, and organic matter have been fully oxidized, which, however, is soon the case if air has access to the surface of the melt.

$\delta$. Fusion with sodium bicarbonate.-C. Holthof, who recommends ${ }^{a}$ this flux strongly, uses 12 to 15 parts of it to 1 of the sample. One quarter of this is placed on the bottom of the crucible, a second quarter is thoroughly mixed with the rock powder in a small warmed dish, and the mixture is then mixed with a third quarter on glazed paper and poured into the crucible. The last quarter is used for rinsing the dish and paper and covering the contents of the crucible, which may be half full. The crucible is heated over a low flame till the bottom is dull red, then during fifteen minutes the flame is increased till the lower quarter of the crucible is dull red, in which state it is maintained for fifteen minutes. Then fusion is brought about by the full flame and maintained for a time. He claims that action between the silicate and carbonate takes place at a lower temperature than with the normal carbonate before fusion, and that for this reason there is almost no spattering onto the lid of the crucible.

$\varepsilon$. Treatment after fusion. - When fusion is complete, the crucible is seized with the tongs (fig. 1, p. 28), and the contents are caused to solidify in a thin sheet over the sides and bottom by imparting an appropriate gyratory motion with the arm during the cooling process. This is preferable to allowing the melt to form a thick cake at the bottom, since much less time is required for disintegration, and separation from the crucible is usually much easier.

The contents of the crucible are placed in a rather tall covered beaker with some water, and hydrochloric acid of 1.1 specific gravity is gradually added in excess. The depth of the evanescent pink color usually produced on addition of the acid allows of judging approximately the amount of manganese present. The beaker is placed on the water bath, and when disintegration is complete, having been assisted by gentle pressure with a blunt glass rod, the contents are transferred to a large platinum dish and evaporated on the bath. It is permissible to dispense with the beaker and to perform the whole

a Zeitschr. anal. Chemie, vol. 23, 1884, p. 499. 
operation of solution in the platinum dish, but there will be greater solution of platinum in this case by reason of the action of the hydrochloric acid on sodium manganate, etc., in direct contact with platinum.

\section{B. SUBSEQUENT TREATMENT.}

From this point the treatment will ordinarily be the same whether the boric-oxide or the sodium-carbonate method of decomposition has been employed.

a. SEPARATION OF SILICA.

$\alpha$. Reasons for adoption of accepted procedure.--The once universal practice of employing a single evaporation with hydrochloric acid for the dehydration of silica has been thoroughly discredited by the work of a number of writers. R. Bunsen ${ }^{a}$ was aware of the impossibility of separating silica by a single evaporation, and he corrected the alumina in his silicate analyses for the silica it was always found to contain. Later, E. Ludwig, ${ }^{b}$ and still later, C. Meineke, ${ }^{c}$ drew renewed attention to the fact and insisted on the importance of correcting the alumina in the manner indicated. Ludwig and, at first, Meineke contented themselves with a single evaporation, and maintained that all of the unprecipitated silica was to be found with the subsequently precipitated iron and alumina. In his second paper Meineke prescribes two evaporations (presumably with intervening filtration) and the rejection of the silica subsequently recovered from the alumina as due to impurity in the ammonia or derived from the vessels, unless these were of platinum. He furnishes quantitative data.

In spite of these publications the practice continued of employing but a single evaporation. Some years later Alexander Cameron, ${ }^{d}$ seemingly in ignorance of the earlier work, reopened the subject with additional quantitative data, and insisted on more than one evaporation with intervening filtration instead of trying to recover the balance of the silica from the alumina. He also showed, as Ludwig had asserted, that a common practice of evaporating to dryness several times with fresh portions of acid without intervening filtrations did not reduce the silica in the filtrate, and that the presence of aluminum, iron, and calcium was without influence on the results; further, that the dehydration was more complete when a high temperature was employed, but that it could not be made complete at one treatment by any modification of the process.

According to J. P. Gilbert, ${ }^{e}$ drying temperatures above that of the steam bath offer no advantage unless much magnesium is present, when the most favorable temperature is $120^{\circ}$. He found that much

a Ann. Chem. Pharm., vol. 61, 1847, p. 265.

b Z,eitschr. anal. Chemie, vol. 9, 1870, p. 321.

$c$ Repert. anal. Chemie, vol. 7, 1887, pp. 215, 757.

$d$ Chem. News, vol. 69,1894 , p. 171.

e Technology Quarterly, vol. 3, 1890, p. 61; Abstract in Zeitschr. anal. Chemie, vol. 29, 1890 , p. 688. 
calcium chloride seems to facilitate dehydration of the silica, but that magnesium chloride above $120^{\circ}$ by decomposing forms a silicate which dissolves in hydrochloric acid and increases the amount of silica carried into the filtrate. He confirmed the earlier belief that drying temperatures higher than that of the steam bath increase the amount of insoluble impurity in the silica, and that this amount can not be overcome by long digestion with hydrochloric acid. Further, he confirmed D. Lindo's statement ${ }^{a}$ that evaporation with sulphuric acid till the appearance of white fumes gives a higher result in silica than with hydrochloric acid. But for general rock analysis the use of sulphuric acid at this stage must be rejected utterly.

Some of the above experiments of Cameron and Gilbert were repeated and confirmed by me. ${ }^{b}$ In the course of this work it was found that hydrochloric acid itself exerts a marked solvent action on silica that has been separated from solution in any of the ways above outlined, whence it becomes plain how a portion of the silica always found in the filtrates gets there, and that it is hopeless to expect to prevent this by a single prolonged drying. Other explanations commonly offered for the observed solubility are the formation of soluble silicates by interaction between the silica and salts present during drying and the protective influence of those salts. The former of these should become more active with increasing temperature, though Gilbert's work does not seem to indicate this except when magnesium is present in quantity.

C. Friedheim and A. Pinagel ${ }^{c}$ prescribe washing the silica with dilute hydrochloric acid instead of hot water because of the marked solubility in water observed by them. But, according to experiments made by myself to test this point, the direction is a vain one, for the results obtained by following it in the ordinary course of rock analysis were worse, if anything, than when pure water was used.

$\beta$. Procedure in absence of notable amounts of fluorine.-Based on the above observations the following procedure is deemed best adapted for general rock analysis, in the absence of more than 0.2 or 0.3 per cent of fluorine.

The evaporating dish should be of platinum and as large as possible to permit considerable surface distribution of the dry mass. Porcelain may be used, but never glass, if platinum is not available. Water or steam-bath temperature suffices, is indeed demanded, for the evaporation to approximate dryness. If the analysis is begun in the morning the first filtration can be made in the late afternoon. Nothing is gained by continuing the evaporation long beyond apparent dryness this first time, for the slight reduction in the soluble silica

a Chem. News, vol. 60, 1889, p. 14 .

$b$ Common errors in the determination of silica: Jour. Am. Chem. Soc., vol. 24, 1902, p. 262; Chem. News, vcl. 86,1902 , pp. 79, 89 . For details of experiments referred to here and on several of the following pages the reader will do well to refer to this paper.

c Zeitschr. anorg. Chemie, vol. 45, 1905, p. 411. 
gained by so doing is offiset by a large loss of time, nor is there much advantage in crushing the residue to powder, whereby, moreover, the dish is easily scratched. The amount of silica passing into the filtrate will generally vary from 1 to 3 per cent of the amount present, and is but little less after twenty hours drying than after one-tenth of that time. $^{a}$ Upon the seemingly dry mass is poured enough strong hydrochloric acid to thoroughly drench it, then an equal bulk of water, and the dish is placed covered on the bath for ten to thirty minutes and the contents occasionally stirred. It is quite permissible and sometimes advisable to defer adding the water till the strong acid has been in contact with the salts for ten or fifteen minutes. More water is then added and filtration proceeded with, first by decantation, leaving nearly all the silica in the bottom of the dish. It is generally in a coarse condition and may be ground finer with a pestle. If there is a good deal of iron in the rock it may be advisable to add hydrochloric acid of half strength and heat, after which the silica is brought onto the filter. It is not recessary to remove at this time the film of silica that may adhere to the dish in spots beyond the power of a stiff feather to remove, for in the subsequent evaporation it will disappear. The washing should be done with cold water or with hot dilute acid till the absence of any yellow color in the precipitate or paper indicates removal of most of the iron. Hot water may with advantage be used for the last washings, and the paper should be sucked dry at the pump.

The filtrate is evaporated again in the same dish to dryness. Usually this state will be reached by the following morning. It may be hastened, if desired, by placing the dish in an air bath at $110^{\circ}$ or $120^{\circ}$. It is probable that a much higher temperature would in the majority of cases have no bad effect except to increase the amount of insoluble matter other than silica. If the data of experiments 6 to 9 in the paper already cited ${ }^{a}$ are conclusive, they show that at this stage thorough drying exercises an appreciable effect in reducing the silica in the next filtrate, though the small amounts there reported when experimenting with pure quartz are not to be expected in rock work. If time is no object, and great accuracy is sought, a third evaporation and filtration should be made, for the silica in the second filtrate will amount to 2 to 4 milligrams. Nearly the whole of this can be recovered later from the alumina, but not all, hence the advisability of securing as complete separation here as possible. The second and if need be third filtrations with their attendant operations consume less time than the first, and, of course, smaller filter papers are to be used. It is to be noted that these later silicas are more colored thar. the first, and if the rock is even moderately titaniferous are by ro means pure silica. 
With minerals like the zeolites, or artificial products such as Portland cements, which are directly soluble in hydrochloric acid, the drying may be very much hastened by placing the covered dish, after bringing to approximate dryness on the bath, on a sand bath or on a triangle which itself rests on a hot plate. The temperature may rise in the dish to $200^{\circ}$; in fact, according to Bertram Blount, ${ }^{a}$ it should not be lower. After an hour's exposure to this temperature less silica will perhaps pass into the filtrate than after prolonged drying at. steam-bath temperature, but this is usually at the expense of greater contamination of the filtered silica. Since this contamination is very large (see e, p. 80) with highly titaniferous and ferruginous rocks and minerals, the operator should use discretion in his choice of a high drying temperature.

$\gamma$. Procedure with rocks and minerals containing fluorine.-This subject has been touched on in commenting on the boric-oxide and sodium-carbonate methods of fusion, ${ }^{b}$ and will be considered in detail under the head of "Fluorine" (p. 155).

\section{b. IGNITION OF SILICA.}

The application of a strong blast ${ }^{c}$ for twenty to thirty minutes with crucible covered is absolutely necessary to expel all moisture from the silica, and the latter is then not hygroscopic. The first of these statements, long extant in the literature, has been disputed by G. Lunge and C. Millberg ${ }^{d}$ on the basis of experiments made on silica obtained by the decomposition of silicon tetrafluoride by water. It has been shown, ${ }^{e}$ however, that, while the objection holds good for silica thus prepared, it does not for silica obtained by the decomposition of an alkali silicate by means of an acid. F. P. Treadwell has confirmed the observation as to the difference in behavior of the two forms of silica, but regards the blast as unnecessary if a Teclu instead of a Bunsen burner is used. My experience is not in agreement with this.

Although the above time limit usually suffices with a powerful blast, and may be materially shortened for small amounts of silica, such as

a Jour. Soc. Chem. Ind., vol. 21, 1902, p. 1217.

$b$ See a, p. 68, and footnote, p. 71 .

$c$ It must be borne in mind that some platinum crucibles lose weight steadily and very appreciably on long blasting, not only when new but even after long use. When a crucible suffers from this defect the rate of loss should be ascertained from time to time and allowance made accordingly, or else the weight of the crucible should be taken after and not before ignition of the precipitate. (See on this subject Hall, R. W., Jour. Am. Chem. Soc., vol. 22, 1900, p. 494.) It was hoped that with the advent of the electric furnace prolonged ignitions would not be subject to this source of error, but it was soon found that at $1,250^{\circ}$, the maximum temperature attainable in the furnace at the Survey, medium-sized crucibles lost approximately 1 milligram in weight per hour. Similar losses have since been noticed and published by others. Their cause seems to be chiefly the distillation of iridium out of the alloy. At one time an error of about the same magnitude in the opposite direction caused perplexity until it was found that the platinum wire in the bedplate of the furnace was burned out. The crucible, then occupying the coolest place in the furnace, received a deposit of some of the metal volatilizing from the wires in the side plates.

$d$ Zeitschr. angew. Chemie, 1897, p. 425.

e Hillebrand, W. F., Jour. Am. Chem. Soc., vol. 24, 1902, pp. 372-373. 
are usually found in limestones and even in cements, there is need occasionally to prolong it in very exact work until repeated weighings give the same value or a loss no greater than that suffered by the crucible itself. ${ }^{a}$

$$
\text { c. CORRECTION FOr IMPURITIES IN THE SILICA. }
$$

The weight of the crude silica should always be corrected for impurities, which are never entirely absent, by evaporating with sulphuric and hydrofluoric acids and again blasting for a minute or less, according to the amount of residue. It is a mistake to neglect another weighing at this point, even if the residue may appear insignificant. In the author's experience it never has been found to be unweighable, and, furthermore, the error due to loss in weight of the crucible itself is eliminated if the weighing is made. The silica in the crucible should be moistened with water before adding the hydrofluoric acid, though with strongly blasted silica the action is not so violent as with that which has not been thus treated. With rocks carrying 60 to 80 per cent of silica one or two drops of sulphuric acid diluted one-half suffice, but with less siliceous rocks the amount ought to be increased, not only in order to surely afford acid enough for the conversion of all the contaminating bases to sulphates, but also to prevent loss of titanium by volatilization as fluoride, since with increasingly basic character of the rocks the percentage of this element in the residue is likely to increase.

Precipitated silica is easily removed by one evaporation with a sufficiency of hydrofluoric acid, but if quartz happens to be present this is not the case, for it is but slowly dissolved and more than one treatment may be necessary.

The subsequent precipitate of alumina, etc., is usually ignited in the crucible containing the residue from the silica.

\section{d. ACCURACy of the silica Determination.}

By observing the above rules-use of piatinum, repeated evaporations and filtrations, proper blast ignition, and correction for foreign matter-the determination of silica, from being one subject to grave error, has become one of the most exact of which I have cognizance, provided precautions are taken to recover, as hereinafter described, the portions that inevitably are to be found with the alumina. With careful work variations of 0.1 per cent are the exception in duplicate analyses.

\footnotetext{
$a$ For the proof see Hillebrand, W. F., Jour. Am. Chem. Soc., vol. 24, 1902, p. 373. The duty of every analyst to test the efficiency of his blast or muffle can not be too strongly emphasized. The discordance of many statements and results on a variety of subjects is to be attributed to a difference in the ignition temperature employed by different writers. A really good blast will readily melt a few centigrams of orthoclase powder in the bottom of a 15-gram platinum crucible, showing a temperature of probably $1,200^{\circ}$ :
} 
e. COMPOSITION OF THE RESIDUE OBTAINED FROM THE SIEICA.

The qualitative composition of this residue varies with different rocks less than its quantity. It will contain alumina and ferric, titanic, and phosphoric oxides invariably if these are present in the rock. If the rock is low in the last three, particularly the last two, the residue should be slight, occasionally less than 1 milligram. It is usually much greater, and may amount to 2 or even 3 per cent with basic rocks very rich in titanium and phosphorus. It is, however, a great mistake to suppose, as some chemists still seem to, that all or nearly all the titanium is to be found with the silica. It may reach one-third of the total amount.

If in correcting the silica, toward the end of the evaporation, when the hydrofluoric acid has been driven off and the sulphates begin to appear in solid form, the residue has a peculiar milky or enamel-like appearance, it may be taken as evidence of much phosphorus and titanium, though possibly the appearance may be due to zirconium with the other elements named. Although observed in but one series of rocks, ${ }^{a}$ the appearance is so unusual and striking that it was deemed worthy of record.

It might be supposed that the residue would contain most of the barium of those rocks carrying that element together with sulphur or sulfates, but in my experience this is not so. Only when there is considerable excess of $\mathrm{SO}_{3}$ over $\mathrm{BaO}$ will any of the latter be present in the residue, and in the vast majority of cases there is none at all. This is due to the appreciable solubility of barium sulphate in hot hydro-chloric acid, coupled with the fact that barium is so minor a constituent of most rocks." Should some of it be present in the residue, its removal and estimation at this stage are not necessary, as it can be more conveniently recovered later, together with the silica accompanying the alumina, etc., precipitate (f. $\beta$, p. 90 ).

Quite as rarely is calcium or magnesium ever a component of the residue if the decomposition of the rock powder was complete at the outset. I have repeatedly proved that the residue, after re-solution, is quantitatively precipitable by ammonia in presence of an ammonium salt.

\section{f. Platinum in Filtrates.}

'The filtrates from the silica always contain notable amounts of platinum. This arises in very small degree from the crucible fusion, unless niter was added; in a larger degree indirectly from the action of hydrochloric acid on the manganate, vanadate, and sometimes chromate of sodium; and, if much iron is present, in no small degree from the reduction of ferric chloride to ferrous by the platinum of the dish. This last reaction has attracted little attention until recently, but is 
mentioned by Gmelin-Kraut, ${ }^{a}$ and can be readily demonstrated by evaporation of ferric chloride in platinum.

The removal of this platinum before precipitating alumina and iron is not necessary (but see third footnote, p. 90), and to do so involves the reoxidation of all iron and subsequent boiling to remove or destroy the excess of oxidizing agent, together with the expenditure of much valuable time. The iron is already oxidized by the fusion, and needs no further help in that direction. Nevertheless, if time is not a prime object, its removal by hydrogen sulphide is to be recommended. In the following descriptions, however, it is assumed that the platinum has not been gotten rid of at this stage.

\section{METALS PRECIPITABLE BY HYDROGEN SULPHIDE.}

The presence in appreciable amounts of metals precipitable by hydrogen sulphide, except perhaps copper, is of so infrequent occurrence in most rocks that discussion is unnecessary in their connection. In case it is necessary to precipitate them at this stage, however, it is always well to bear in mind that a little titanium may be thrown down along with them. Separations of the silica should be made in porcelain, to eliminate platinum; or, better still, the quantitative determination of these metals should be made in a separate portion of the rockbroken up by the action of hydrofluoric and sulphuric acids.

\section{ALUMINUM-TOTAL IRON.}

\section{A. INDIRECT METHOD FOR ALUMINUM. (TITANIUM.)}

a. PRELIMINARY REMARKS.

The difficulties in the way of successfully producing and handling the complex precipitate which is obtained in the next stage of the analysis and which should contain the aluminum, iron, titanium, and phosphorus, besides chromium, vanadium, zirconium, and rare earths if present, and according to some preferences the manganese, are very great. It is fully recognized that there are objections to parts of the procedure that is usually followed in this laboratory, but the same may be said of any other known procedure, including that preferred by Dittrich. It is believed that the Survey method, which in its main features is closely modeled after old and well-known methods, will in competent hands give better results on the whole than any other.

The common practice in this laboratory is to find alumina by difference, after deducting from the precipitate produced by ammonia or:

Bull. 305-07-6 6 
sodium acetate the sum of all other oxides this precipitate may contain. Of these, only ferric oxide, titanic oxide, and the trace of silica are determined in this portion (see also third footnote, p. 90), those of phosphorus, vanadium, chromium, zirconium, and sometimes manganese being looked for in other portions of the rock powder. This throws on the alumina all errors involved in their separate determinations; but these may balance, and in any case the probable error can hardly be as high as that involved in the direct weighing of the alumina itself, considering the difficulty of effecting a satisfactory separation of it from all the other admixtures, an operation which would, moreover, immoderately extend the time required for each analysis.

b. PRECIPITATION OF ALUMinUm, IRON, ETC., WITHOUT PRECIPITATING MANGANESE.

$\alpha$. Precipitation by ammonia.-Two precipitations by ammonia at boiling heat are usually quite sufficient to separate iron, aluminum, phosphorus, vanadium, chromium, titanium, and zirconium, if all these are present, from nickel, manganese, the alkaline-earth metals, and magnesium, provided ammoniacal salts are present in sufficient quantity. This last point is of special importance as regards magnesium, and failure to observe it is doubtless the reason why many old analyses, and sometimes modern ones, show utterly improbable percentages of alumina, especially as chemists were formerly often satisfied with a single precipitation. The necessary ammonium chloride is better obtained by the use of purified ammonia water and hydrochloric acid than by the addition of the solid salt, which is seldom pure. The chemist should satisfy himself what amounts of ammonium salt are needed to hold in ammoniacal solution the maximum percentage of magnesium that the rock he is analyzing might contain. With extremely high magnesian rocks a third precipitation is generally requisite.

The precipitations are made in a bulk of 300 to $400 \mathrm{~cm} .^{3}$, at boiling heat, in a platinum dish if possible, otherwise in one of the borosilicate beakers of the Jena or Nonsol type. The ammonia should be as free from carbonate as possible, as well as from any nonvolatile matter, and it need not be added in any great excess. The complete boiling off of this excess is unnecessary, as pointed out by Genth and Penfield, since it is apparently the washing with pure water and not the free ammonia which carries small amounts of alumina into the filtrate. S. L. Penfield and D. N. Harper ${ }^{a}$ recommend washing with a dilute solution of ammonium nitrate $\left(20^{\prime} \mathrm{cm} \cdot{ }^{3}\right.$ nitric acid, neutralized by ammonia, to the liter), and also the solution of the first precipitate in nitric instead of hydrochloric acid, in order to shorten the washing, 
there being no chloride to remove, both of which are excellent suggestions. ${ }^{a}$

For a reason mentioned under e (p. 87) the second precipitation may with advantage be made in the presence of macerated filter paper, as recommended by Dittrich. ${ }^{b}$

In the presence of little or no manganese, and also in the absence of iron, the ammonia method of precipitation is preferable by far to the basic-acetate method described in the following section, but it will occasionally happen that the separation from even very small amounts of manganese is altogether incomplete, and the uncertainty of insuring this separation led me to employ the basic-acetate method for the first precipitation in all cases where manganese is present-and the exceptions are few-even though the precipitation of alumina is sometimes less complete than by ammonia and in spite of other admitted defects, as, for instance, a tendency of the precipitate to run through the filter on washing. ${ }^{c}$

The most likely explanation of the persistent retention of small amounts of manganese by the precipitates produced by ammonia or acetates, even after repeated precipitation, seems to be that a portion of the manganese becomes oxidized to the $\mathrm{Mn}_{2} \mathrm{O}_{3}$ state and remains so when redissolved, being then in a fit condition for reprecipitation.

$\beta$. The basic-acetate precipitation. - Although for this precipitation most writers seem to prescribe or prefer sodium acetate, except when the presence of a fixed alkali would interfere with subsequent operations, there does not seem to be any valid reason why ammonium acetate should not do quite as good service, besides having the advantage of easy removability afterwards in case of need. Also there is probably no good reason why the corresponding carbonates instead of the hydroxides should be used for neutralizing the hydrochloric solution before adding the acetate, other than the fact that they were, when the methods originated, and still are, easier to obtain comparatively free from silica and alumina. It is to be borne in mind that with precipitates low in iron and high in aluminum the separations are less satisfactory than when the reverse condition prevails. In rocks, alumina usually predominates, often largely, over iron oxide, so

\footnotetext{
$a$ A single objection, and this of a practical nature, weighs against these suggestions. It will not do to evaporate to dryness filtrates containing both chlorides and nitrates in the same platinum dish, still less to drive off the mixed ammoniacal salts in platinum. With much iron present the final precipitation from a nitric-acid solution is a decided advantage, but is less so in the case of alumina free or nearly free from iron. Contrary to numerous statements and general belief, there is no danger of loss of aluminum chloride by volatilization when the precipitate is ignited beforecomplete removal of ammonium chloride.

${ }^{b}$ Anleitung zur Gesteinsanalyse, 1905, pp. 10, 11, 14; Ber. Deutsch. chem. Gesell., vol. 37, p. 1840, 1904.

$c$ The fact must not be overlooked that certain of the rare earths may pass completely in to the filtrate if the basic-acetate method is followed. If then, later, on rendering the combined filtrates ammoniacal an unexpectedly large piecipitate appears, this should be carefully examined as to its nature. In an analysis of piedmontite from Maryland over 2 per cent of rare earths, including cerium and others not identified, were quantitatively separated in this way from iron, alumina, etc.
} 
that none of the methods of making the basic-acetate separation that involve the presence of notable quantities of free acetic acid are permissible, because of the solubility of aluminum hydroxide in this acid even when it is very dilute.

The cold and not excessively acid solution, not exceeding $100 \mathrm{~cm} .^{3}$ in bulk and contained in a beaker of Jena or Nonsol glass, is first neutralized with great care by adding a freshly prepared solution of sodium carbonate, drop by drop. When the solution begins to deepen in color, the addition of the carbonate is continued with less speed and with longer stirring between the successive drops. When, now; the precipitate that each drop causes begins to dissolve very slowly, no further addition may be made till the liquid becomes quite clear again. With practice, it is not difficult to recognize this condition, even when the liquid is very dark. The intensity of the color will depend on the amount of iron present and the degree of dilution; therefore with small amounts of iron one is much more liable to overstep the end point than with solutions rich in iron. When, in spite of vigorous stirring, the turbidity after a final drop seems rather to increase than diminish, one or, if need be, two drops of acid are added. If this does not clear the liquid, it is best to add a slight excess and to repeat the neutralizing process.

Two or three grams of sodium acetate dissolved in a small amount of water are now poured in and then boiling water till the total bulk is about $400 \mathrm{~cm}^{3}$. The beaker is placed over a lamp and its contents brought to boiling and kept in that state for two or three minutes. As soon as settling has taken place filtration is proceeded with. It is generally best to dispense with the pump at this stage and to use a filter of such size that the whole of the precipitate can be conveniently brought upon it without filling to the top. The washing is to be done with hot water, to which has been added a very little sodium acetate, for the purpose of guarding against an otherwise inevitable turbid filtrate.

The precipitate, having been sucked dry at the pump, is redissolved in hydrochloric acid, reprecipitated by ammonia as in $\alpha$, washed, sucked dry, and treated as in e (p. 87), after recovery as in d (p. 86) of the portions of aluminum and iron that may have passed into the filtrates. ${ }^{a}$

\footnotetext{
a Mittasch's method for the basic-acetate separation of manganese from iron.-A study of the conditions necessary for successful separations of large or small quantities of manganese from iron by one treatment has been made by A. Mittasch ("/eitschr. anal. Chemie, vol. 42, 1903, p. 492), who gives the following directions for the use of ammonium acetate. It must be borne in mind, however, that this method is not applicable to aluminous materials.

The solution, containing not much free acid (hydrochloric) and preferably cool, whose volume should not exceed $100 \mathrm{~cm} .{ }^{3}$, is neutralized in a beaker with concentrated ammonium-carbonate solution( 200 grams per liter, about double molar) till the precipitate that is formed begins to redissolve slowly, then with weaker solution (about one-tenth molar) till a slight precipitate persists for one or two minutes in spite of stirring. Next is added, according to the amount of this precipitate, 3 to $5 \mathrm{~cm} .3$ of acetic acid (double normal) if the commercial article of ammonium acetate, which nearly corresponds to the
} 
c. PRecipitation of IRON, aluminum, etc., TOGéther with manganese.

$\alpha$. By ammonia and ammonium persulphate.-This method has for its object the coprecipitation of manganese with a view to simplifying subsequent operations. It is, however, defective in case the rock carries appreciable amounts of barium and strontium or is very high in calcium, by reason of the certain partial precipitation of some of one or both of the first two as sulphates and its possibility in the case of calcium. It is probable that calcium can be completely held in the filtrates by two precipitations in analyzing almost any silicate rock, but this is not at all certain with respect to limestones, a point that has not yet been investigated. If neither strontium nor calcium is present in sufficient amount to be retained in the precipitate, there is no vital objection to the method on this score, for barium can be recovered, as in f. $\beta$, page 90 .

The applicability of the method as above limited being assumed, it may be carried out as follows, though too few tests have thus far been made in this laboratory to warrant its unqualified recommendation.

To the filtrate from the silica contained in a platinum dish, and in bulk from 100 to $200 \mathrm{~cm} .{ }^{3}$, is added enough hydrochloric acid to prevent precipitation of manganese when made ammoniacal (see b. $\alpha$, p. 82). Heat is applied, and about 1 gram of ammonium persulphate $^{a}$ free from all interfering impurities is added . When the liquid

acid salt $\left(\mathrm{NH}_{4}\right) \mathrm{C}_{2} \mathrm{H}_{3} \mathrm{O}_{2} \cdot \mathrm{C}_{2} \mathrm{H}_{4} \mathrm{O}_{2}$, is used, or $10 \mathrm{~cm} .{ }^{3}$ if the normal salt is used. This last is made by neutralizing acetic acid with ammonia. The solution is then diluted with water to about $400 \mathrm{~cm} .{ }^{3}$ and brought nearly to the boiling point. Any precipitate that may have appeared being disregarded, 20 $\mathrm{cm} .{ }^{3}$ of the acid acetate (one-half molar) or $5 \mathrm{~cm} . .^{3}$ of acetic acid and $10 \mathrm{~cm} \cdot{ }^{3}$ of the normal acetate (molar) is added, and the boiling temperature is maintained for a minute longer. Precipitation is complete even at $60^{\circ}$, but filtration is easier if the boiling temperature is reached. As soon as the solution is settied filtration is begun and the washing performed with hot water at first, then with hot water containing some acetate and a little acetic acid. Any adhering film is removed from the glass with hydrochloric acid and represipitated with ammonia. If this is added to the main filter it must not be done till the large precipitate has been washed completely, because of the danger of precipitating, by the ammonia. manganese that has not jet been washed out.

The Brunck-Funk separation of iron as basic acetate from manganese, zinc, nicleel, and cobalt.-The following method of O. Brunck (Chem. Zeit., vol. 28, 1904, p. 514), slightly modified and extended by W. Funk (Zeitschr. anal. Chemie, vol. 45, 1906, p. 181), is claimed to afford a complete separation by one treatment. Its applicability to aluminous materials has, however not been tested, and it is quite improbable that it would work with rocks containing phosphorus and titanium.

The method differs from other modifications of the acetate process in the avoidance of the delicate neutralization, by removing excess of acid by evaporation and in the addition of potassium chloride to form a double salt with ferric iron, and thus prevent the separation of a basic chloride during the drying. As given by Funk for materials soluble in acids the procedure is as follows:

To the chloride solution in a large dish is added potassium chloride (about 0.35 gram to $0.1 \mathrm{gram}$ of iron), the solution is evaporated to apparent dryness on the steam bath, but without expelling all of the free acid. The residue is broken up superficially and the drying on the bath continued for five to ten minutes. It is then taken up in 10 to $20 \mathrm{~cm} .^{3}$ of cold water, with which it should give a clear solution. It is requisite that there shall remain a slight amount of free acid to set free acetic acid from the acetate in the next stage of the operations. One and a half to two times the theoretically needed quantity of sodium acetate ( 3 molecules of $\mathrm{NaC}_{2} \mathrm{H}_{3} \mathrm{O}_{2} \cdot 3 \mathrm{H}_{2} \mathrm{O}$ to 1 atom of iron) is now added, in a solution which has been rendered slightly acid with acetic acid if originally alkaline. After diluting with cold water (to 400 to $500 \mathrm{~cm} .^{3}$ for 0.2 gram iron) the solution is gradually heated with stirring to the point of precipitation $\left(60^{\circ}\right.$ to $\left.70^{\circ}\right)$, allowed to settle, decanted, collected on the filter, and washed with hot water.

$a$ This reagent as sold must be purified. Jannasch (Prakt. Leitfaden d. Gewichtsanalyse, 2d ed., 1904, p. 179) accomplishes this as follows: To a saturated warm solution (not over $95^{\circ}$ ) ammonia is 
begins to boil, ammonia is added carefully till in decided excess, as shown by the odor, and boiling is kept up for several minutes. The precipitate is allowed to settle and is quickly filtered and washed with hot water three or four times and sucked dry by the pump. It has not been determined if such a very dilute solution of persulphate has any appreciable effect on glass, but apparently not, to judge from my own limited experience. The precipitate is then dissolved in a small amount of hot hydrochloric acid containing a few drops of sulphurous acid, and after addition of well-macerated filter paper reprecipitated precisely as the first time. It may then be washed three or four times with hot water containing a few drops of ammonia, then ignited in the paper, and weighed as $\mathrm{Al}_{2} \mathrm{O}_{3}, \mathrm{Fe}_{2} \mathrm{O}_{3}, \mathrm{Mn}_{3} \mathrm{O}_{4}, \mathrm{TiO}_{2}$, and $\mathrm{P}_{2} \mathrm{O}_{5}$. Any chromium in the rock should be in the filtrates, wholly oxidized by the persulphate, but it will be recovered with the unprecipitated traces of alumina, as detailed under d. $\gamma$, page 87 , and should be added to the main precipitate above. It is not best to attempt to determine the chromium colorimetrically in the filtraite.

B. By ammonia and ammonium sulphide.-Although this old method of precipitation can be employed in special cases, it is not well adapted generally to silicate rocks, and will, therefore, not be described in this connection.

d. RECOVERY OF IRON AND ALUMinUm From the FILTRATES.

$\alpha$. After an ammonia precipitation.-The filtrates are evaporated, always in platinum if possible and allowable, to a small volume. If ammonium salts are present in abundance, it is advisable to evaporate the filtrates separately, the second to dryness. Its dry contents, almost wholly ammonium salt, are expelled by heat, the slight residue being taken up with hydrochloric acid and added, whether whoily soluble or not, to the other filtrate reduced in bulk. To this ammonia is now added and the evaporation continued, the solution being kept alkaline, in order to coagulate the small amount of alumina (and usually iron hydroxide) that will invariably be found. This is collected on a small filter and the filtrate caught in a flask of 150 to $200 \mathrm{~cm} .^{3}$ capacity, if manganese is to be determined, otherwise in a platinum dish of suitable capacity for the calcium precipitation. The small precipitate is washed with hot water, burned, and added to the main one.

$\beta$. After a basic-acetate precipitation.-The first filtrate is evaporated to, or nearly to, dryness, in order to get rid of the excess of

added till its odor persists. [More will have to be added at intervals, since the continual decomposition of persulphate sets free sulphuric acid.-W.F.H.] When the precipitate of alumina, etc., has settled, the solution is filtered as rapidly as possible through asbestos, a porcelain funnel being used, since the strong solution attacks both paper and glass markedly. When cooled to about $30^{\circ}$, the clear filtrate is poured into twice its volume of alcohol and further cooled by ice appleed externally. After half an hour the precipitated persulphate is collected on a filter, washed with alcohol, and then with ether, and dried in the air. The alcohol must be thoroughly removed. A commercial article purified thus by me (W.F.H.) was found to contain no interfering impurities, though not free from alkali. 
acetic acid. The residue is then taken up, with only enough hot water to keep it in solution, and the dish is replaced on the bath for a short time. The precipitate, which will generally be appreciably larger than that obtained in $\alpha$, is collected on a small filter $(7 \mathrm{~cm}$.), and the filtrate in a flask of 150 to $200 \mathrm{~cm}^{3}$ capacity. The second or ammoniacal filtrate, having been separately evaporated to small bulk, with the addition of a few drops of ammonia toward the end, serves as first wash water for the other dish and for the precipitate on the filter. Both dishes and the filter are finally rinsed with hot water. The ammoniacal filtrate contains enough ammonium salts to prevent precipitation of magnesium in the first filtrate when the two come together.

If manganese peroxide has been deposited on the surface of the dish it is removed by hydrochloric acid and a drop or two of sulphurous acid, which mixture is then passed hot through the filter. A reprecipitation by ammonia is then made, and the precipitate collected again on the filter and added to the main one, the filtrate passing into the flask containing the previous filtrate. If much manganese is present, of course a second precipitation by ammonia of the small precipitate may be required. In these cases there is no difficulty in getting all the 'manganese into the filtrate.

$\gamma$. After precipitation by ammonia and ammonium persulphate.The filtrates are evaporated as in $\alpha$. The second is carried to dryness and the ammoniacal salts are removed by ignition. The residue is treated with a little hot hydrochloric acid and added to the first. A drop or two of sulphurous acid is then added if chromium is present, as will usually be shown by a yellowish color of the solution. The aluminum, chromium, and trace of iron are then precipitated by ammonia in slight excess and the digestion allowed to proceed on the bath till the slight precipitate has coagulated. This is collected on a small filter $(7 \mathrm{~cm}$.) and washed with hot water, the filtrate being collected as in $\alpha$ and $\beta$ if nickel or zinc are to be looked for, otherwise in a large platinum dish for treatment as in 10 (p. 101).

The precipitate is redissolved in hydrochloric acid, reprecipitated in small bulk of solution by ammonia, and finally added to the main precipitate obtained in c, page 85.

e. ignition of the pRecipitate of tron, aluminum, etc., oxides.

The combined precipitates of alumina, etc., obtained in either $a, b$, $\mathrm{c}$, or $\mathrm{d}$, are ignited without preliminary drying in the crucible containing the residue from the silica (6. B. c, p. 79), unless considerable iron is present. In that case the main precipitate is dried and removed as far as possible from the paper, which is ignited separately to prevent partial reduction of a portion of the iron oxide. This is especially necessary when the precipitates were obtained without 
using macerated filter paper, in which case they are, after ignition, lumpy, and any magnetic oxide that may have been formed can not be wholly reoxidized by heating or by treatment with nitric acid (see 5. A, p.59). They are also slow to yield to subsequent treatment. A precipitate formed in presence of thoroughly macerated paper, as recommended by Dittrich, on the other hand, yields on ignition a powder, and the fine state of division makes reoxidation of any reduced iron easy, besides wonderfully shortening the time required for subsequent re-solution.

Alumina in the quantities ordinarily found can not be fully dehydrated by the full heat of the Bunsen burner. It must be blasted for five or ten minutes. If iron is present in large amount this last operation must be conducted so as to insure access of air to the crucible (p. 73).

For ignitions of this kind, which involve no very prolonged heating, an electric furnace is admirably adapted by reason of its nonreducing atmosphere, provided the temperature is not high enough to cause by temperature alone reduction of ferric to magnetic oxide of iron.

The empty weight of the crucible, which may have changed since igniting the silica in it, is found after treatment of its contents as in the next section.

f. RECOVERY OF SILICA AND POSSIBLE BARIUM IN THE ALUMINA PRECIPITATE.

$\alpha$. Silica.-The weighed precipitate is transferred as far as possible to another crucible of 25 to 30 grams weight. The small adhering residue, chiefly that originally derived from the silica, is brought into solution by fusion with a small amount of sodium or potassium pyrosulphate. This fusion takes but a few minutes, and must not be continued beyond the time actually needed, otherwise it will be impossible, after cleaning and igniting, to get the correct weight the crucible possessed after the ignition of the alumina. ${ }^{a}$ The hot liquid is poured onto the main mass of precipitate in the other crucible, more pyrosulphate $^{b}$ is added (up to 7 grams in all) and the fusion is renewed and continued until the whole has been dissolved, which in the case of the potassium salt is always easy to be seen, even when the liquid appears dark red and opaque, by removing the crucible from the flame to a good light and allowing to cool. At one point in the cooling the liquid becomes transparent and allows the bottom of the crucible to be seen. This is less the case with the sodium salt.

$a$ It will be remembered (see page 78, footnote $c$ ) that long blast or furnace ignition of the silica may have reduced the weight of the crucible by an appreciable amount, hence the need for getting its empty weight at this point.

$b \mathrm{~A}$ chief objection by many to the use of an alkali bisulphate for bringing this precipitate nto solution is that so much care is needed during a fusion to prevent frothing and boiling over, which involves a very gradual application of the heat and consequent greater expenditure of time. The objection is 
When all is dissolved ${ }^{a}$ the melted mass is poured into a large, dry platinum dish, what adheres to the two crucibles is removed oy diluted sulphuric acid, and the solution, with rinsings, is poured into the dish. Much more dilute sulphuric acid is added and the dish heated till the solid is dissolved. The solution is evaporated as far as can be done on the steam bath, then gradually heated higher till fumes of sulphuric acid come off copiously. During this heating the mas: is apt to darken from reduction and separation of dissolved platinum: The acid should have been used in such quantity that after cooling the mass is pasty and not solid, for it then dissolves readily, on heating; in the water, which is now to be added, the sodium salt dissolving much more readily than the potassium salt.

The dish is placed on the bath and soon the silica can be seen in coagulated form at the bottom. It is collected on a small filter, well washed with hot water, burned, and weighed in a platinum crucible. Being rarely pure, it has to be corrected by a few drops of hydrofluoric and a single drop of sulphuric acid, and reweighed. The slight residue in the crucible is brought into solution by pyrosulphate and added to the main portion, for the treatment of which see $B$, page 90 , after filtering from barium if present ( $\operatorname{see} \beta$, p. 90).

The amount of silica thus recovered varies from 2 to 4 milligrams after two filtrations of the main silica, and is, of course, to be added to this.

Only in this way is the full amount of the silica in the alumina precipitate to be recovered. ${ }^{b}$ Formerly it was customary to regard that remaining undissolved when the pyrosulphate melt was taken up with

sound as applied to the bisulphate, butis swept away if the latter is first converted into pyrosulphate, which is always its condition after the frothing has ceased. To effect this change it is only necessary to melt a large amount of the salt in a platinum dish and keep it in fusion till spattering ceases and white fumes begin to come off freely. If the liquid is then poured out into other dishes it cools in thin sheets or cakes, which can be readily broken up and bottled for future use. With such material the fusion is always tranquil, and the action on the alumina, etc., much more intence and speedy than with the ordinary bisulphate. If a good article of the latter is not to te had ky purchase, it is only necessary to mix and melt together equivalent weights of sulphuric acid and r ure normal sulphate until the water of the acid has been mostly expelled and the conversion to pyrosulphate accomplished.

Of late, the recommendation of J. Lawrence Smith (Am. Jour. Sci., 2 d ser., vol. 40, 1865, p. 248) to use the sodium instead of the potassium salt has been followed with satisfaction. This salt acts more quickly and forms a more soluble double salt with aluminum, but has the slight dicadvantage that the progress of decomposition of the ignited mass is not so readily followed because of the greater tendency of the sodium salt to crust over.

A further objection is the action on the crucible of the fused pyrosulphate and the consequent necessity of removing the dissolved platinum. To obviate this and likewise to effect more speedy solution of the oxides E. Deussen (Zeitschr. anorg. Chemie., vol. 44, 1905, pp. 423-426), fuses the f.nely powdered oxides with acid potassium fluoride (about 1 gram). The mass melts at first over a low flame, then solidifies. Its solution is evaporated with sulphuric acid to expel most of the fluorine, then reduced with hydrogen sulphide or sulphurous acid and titrated for iron. The use of the fluoridei, howerer, incompatible with accurate work in silicate analysis, since the silica in the ignited oxices can not be determined. Moreover, the oxides must be finely powdered and consequently an aliquot part only can be taken for the fusion. The expulsion of fluorine by sulphuric acid mast furthermore be thorough if titanium is to be determined in this solution.

$a$ Should a small portion of the presipitate have escaped solution no particular harm is done. The further treatment is not thereby changed.

$b$ For proof see Jour. Am. Chem. Soc., vol. 24, 1902, pp. 368-370. 
water or dilute acid as the total amount. It is', however, but a small portion of what the alumina held, the greater part having formed alkali silicate during the fusion and remaining in solution afterwards, unless set free and rendered insoluble by the treatment above given.

The silica thus recovered does not, however, represent quite all of that which originally escaped separation by evaporation with hydrochloric acid. A small part (roughly 1 to 2 milligrams for a single precipitation and a total of 1 centigram of silica in the experiments detailed elsewhere ${ }^{a}$ ) is not precipitated with the alumina and escapes recovery altogether. It is therefore apparent why a thorough separation of silica at the start is so urgently called for.

$\beta$. Barium.--Should by any chance the alumina precipitate have held barium sulphate, something which has not happened in my experience with rocks, unless ammonium persulphate was used as in c. $\alpha$, page 85 , it will be found as a contaminant of the silica recovered after the pyrosulphate fusion. The weighed residue remaining after expulsion of this silica by hydrofluoric acid is dissolved (except the platinum it may have held) by a little pyrosulphate and the cooled melt redissolved in dilute sulphuric acid. Any barium will then be left in the form of sulphate, ${ }^{b}$ and can be separated by flotation from the heavier platinum.

B. DETERMINATION OF IRON IN THE PRECIPITATE OF ALUMINA, ETC.

a. Without Regard to the PRESence of VANadium.

$\alpha$. Reduction of the iron.--Into the strongly acid and cool filtrate obtained in f. $\alpha, \beta$, pp. $88-90$, held in a beaker, is introduced hydrogen sulphide for the reduction of the iron to the ferrous state and the precipitation of the platinum resulting from the pyrosulphate fusion. This last effect is rendered complete by causing the solution to boil ${ }^{c}$ with continued passage of the gas, whereby the sulphur becomes coagulated and readily filterable. The gas current is not interrupted till

$a$ Jour. Am. Chem. Soc., vol. 24, 1902, p. 369.

$b$ Some years ago, in a series of analyses of rocks from the Leucite Hills, in Wyoming, there was obtained at this stage, when it was customary to dissolve the melt in cold water preliminary to precipitation of titanium by boiling the neutralized sulphuric solution in presence of sulphur dioxide, a white, more or less flocculent residue which amounted to 1 to 3 per cent of the rock and which was at first taken to be a mixture of tantalic and columbic acids. Eventually, it was found to consist apparently of nothing but $\mathrm{TiO}_{2}$ and $\mathrm{P}_{2} \mathrm{O}_{5}$, with perhaps a little $\mathrm{Z} \mathrm{rO}_{2}$. By repeated fusion with acid potassium sulphate and leaching with cold water it could be gradually brought into solution. It was these rocks which furnished the most striking instance of the peculiar milky sulphate residues mentioned on p. 80 as derived from the ignited silica.

Knop (Zeitschr. Kryst., vol. 10, 1885, p. 73) seems to have obtained a similar mixture in analyzing minerals from the Kaiserstuhl in Baden, but its nature was not ascertained, though it was suspected to be, if not silica, columbiferous titanic acid.

$c$ It may be mentioned that the precipitation of platinum from a hot sulphate solution is far quicker and cleaner than from hydrochloric acid. Further, this platinum sulphide, when ignited in the crucible in which the bisulphate fusion was made, should weigh together with the crucible itself what the latter weighed before the main silica precipitate was ignited in it; in other words, the weight of the platinum recovered by hydrogen sulphide should equal the loss in weight of the crucible due to attack by the bisulphate. In somewhat rare instances this will not be so, but the weight will be greater, showing a 
the solution has cooled somewhat. This is then filtered ${ }^{a}$ warm into a half-liter flask, hydrogen sulphide is again introduced for a few minutes, with production of slight milkiness if any iron had become oxidized during filtration, the flask is then connected with a carbondioxide generator already in action for a time, and the solution brought to boiling to expel the hydrogen sulphide. The expulsion is aided by a rapid current of carbon dioxide, ${ }^{b}$ the end point being found by occasional testing of the escaping gas with lead-acetate paper. $^{c}$ When this point is reached the flame is removed and the flask allowed to cool without interrupting the gas current. The cooling is hastened by placing the flask in a basin of cold water.

$\beta$. Titration of the iron.-A burette with permanganate solution having been made ready, the gas tube is rinsed into the flask and the permanganate at once run into the latter without further dilution of the solution, which is kept in rotatory agitation by an appropriate movement of one hand. The bulk of the solution will be from 100 to $200 \mathrm{~cm}^{3}$. The drops of the titrating fluid that may fall upon the neck of the flask are washed down by aid of a wash bottle. The strength of the permanganate solution would best not much exceed

gain in platinum which may amount to a milligram. Tests have shown that this is not due to retention of platinum by the main $\mathrm{Al}_{2} \mathrm{O}_{3}$, etc., precipitate; hence, it must come from platinum mechanically loosened from the dish during the drying and powdering of the silica preparatory to its collection on the filter, or to some insoluble compound of platinum formed during evaporation and drying of the silica. It may also be in part or wholly due to contamination from reduction of platinum during evaporation of the filtrate from the basic-acetate separation. It will be remembered that from this filtrate a small amount of iron and alumina is recovered and added to the main precipitate. Hence it is always well in fine work to collect the sulphide and weigh the platinum in the original crucible, deducting any excess from the alumina, or else to get rid of the platinum by hydrogen sulphide before proceeding to the precipitation of alumina, etc. (See 7, p. 81.)

$a$ Filtration is not necessary if only precipitated sulphur and no sulphides are in suspension, since this is without reducing action on cold permanganate solution, as H. A. Wells and W. L. Mitchell, and others before them, have pointed out. The above authors used this method of reducing ferric iron in titanic iron ores. (Jour. Am. Chem. Soc., vol. 17, 1895, p. 78; Chem. News, vol. 73, 1896, p. 123.) The observation of A. Coppadoro (Gazz. chim. ital., vol. 31, pt. 2, 1901, p. 217) that the precipitate of sulfur caused by reducing ferric solutions with hydrogen sulphide carries some iron as sulphide, even in strongly acid solutions and after thorough washing, need not be regarded. He seems to have operated on very concentrated solutions as compared with those here in question.

$b$ The gas generated from every new lot of marble must be carefully tested for hydrogen sulphide. Even pure white marble is frequently not free from soluble sulphides-probably pyrrhotite, sphalerite, or galena-and the amount may be enough to appreciably affect the result of titration for iron. A good precaution to take is to wash the gas by passing it through a large $U$ tube containing glass beads and a solution of copper sulphate.

$c$ The test is most delicate when the flask is provided with a two-hole stopper fitted with inlet and outlet tubes of glass. If the outside opening of the outlet tube is small the concentration of the escaping steam and gas contributes to the sharpness of the test. Because of the possibility of the introduction of organic matter from the stopper by the action of the hot steam, it is safer to employ a cap of platinum foil with two holes, one for the gas entry tube, the other for its escape in a concentrated stream.

If the solution was free from milkiness at the start the operation of expelling hydrogen sulphide need not take more than ten to twenty minutes; otherwise a longer time will be required to remove the last traces. The immediate effect of boiling on any moderate amount of suspended sulphur is to coagulate it and clear up the solution completely. Some of the sulphur volatilizes with the steam, but a further portion appears to be converted by it to hydrogen sulphide, so that a faint reaction for that gas can be obtained as long as any sulphur remains. This is probably the explanation for Washington's direstion (Manual of the Chemical Analysis of Rocks, 1904, p. 112) to boil for about two hours, since by his method of reducing the iron, without coagulating the sulphur, the filtrates are always strongly milky. 
$0.0(25$ gram of iron to the cubic centimeter, for the amounts of iron to be determined are moderate and often very small.

The results are strictly accurate, with the limitations set forth in the paragraph below, when care is taken with the reduction by hydrogen sulphide. The method is altogether superior to that involving the use of zinc, since no foreign impurity affecting the result is introduced, and the ever present titanium is not affected, nor is vanadium reduced below the condition of $\mathrm{V}_{2} \mathrm{O}_{4}$, whereas nascent hydrogen converts it, in part at least, to $\mathrm{V}_{2} \mathrm{O}_{3}$.

$\gamma$. Further treatment of the solution after titration.--The titrated solution is returned to a dish, which may be of porcelain, and evaporated to a bulk of much less than $100 \mathrm{~cm}^{3}$ if the rock is supposed to contain less than 1 per cent of titanium. For each additional 1 per cent of titanium the final bulk may be larger by $100 \mathrm{~cm} .^{3}$. The solution is transferred to a graduated flask with a capacity of 50,100 , or $200 \mathrm{~cm}^{3}$ or more, as the case may be, enough hydrogen peroxide free from fluorides is added to fully peroxidize the titanium, the flask is filled to the mark and well shaken, and the titanium is determined colorimetrically (see p. 110).

b. HAVING REgaRd TO THE PRESENCE OF VANADIUM.

If vanadium is present the value found for iron will be in error by the amount of permanganate required to oxidize $\mathrm{V}_{2} \mathrm{O}_{4}$ to $\mathrm{V}_{2} \mathrm{O}_{5}$. . The amount of the correction will differ according as titration of the iron is made after reduction by hydrogen sulphide or nascent hydrogen. If the former is used, as should always be the case, because of the ever present titanium, the vanadium is reduced by it to $\mathrm{V}_{2} \mathrm{O}_{4}$, which in its action on permanganate is equivalent to two molecules of $\mathrm{FeO}$, while the reduction goes further with hydrogen. After the first transitory pink blush throughout the liquid, the more slowly acting vanadium may require the addition of a drop or two more of permanganate before a comparatively permanent coloration appears.

When the amount of vanadium in the rock is known a correction can be applied on the assumption that practically all the vanadium is here collected, a point that needs further investigation. Various authors assert its precipitability with alumina and iron by ammonia and ammonium acetate, though Carnot ${ }^{a}$ states that repeated precipitation by ammonia, ammonium carbonate, or ammonium sulphydrate separates it from iron. My experience with ores very rich in vanadium. shows that precipitation along with iron and aluminum is only partial. Ridsdale ${ }^{b}$ has determined its precipitability with various metals and gives numerous figures which show an approximation to 90 per cent thus thrown down under the conditions prevailing in analysis of

$a$ Comptes rendus, vol. 104, 1887, p. 1803; Zeitschr. anal. Chemie., vol. 32, 1893, p. 223.

$b$ Jour. Soc. Chem. Industry; vol. 7, 1888, p. 73. 
iron slags, the remainder passing into the filtrates and appearing in small part with the lime and to a greater extent with the magnesium phosphate. For all practical purposes it is probably safe to assume that the small amounts of vanadium met with in rocks are wholly in the alumina precipitate.

If the amount of vanadium in the rock is not known and great accuracy is necessary, caution requires the determination of the total iron to be made either in a separate portion or after reprecipitation from the above solution, as follows: Fuse with sodium carbonate, extract with water, bring the insoluble residue into sulphuric solution, reduce, and titrate as above directed. But unless a certain precaution is here observed an error greater than that which it is designed to avoid will be committed. Contrary to general belief the aqueous extract from the sodium-carbonate fusion carries a small but appreciable fraction of a per cent of iron; as I have repeatedly found by actual test. This iron is thrown out with the alumina (and silica, if present) by the usual methods of neutralizing the alkaline solution, and can be brought to light when the precipitate thus formed is treated with a fixed caustic alkali, or again fused with sodium carbonate and leached with water, when it remains wholly or in part undissolved. Hence it is necessary to collect this iron and add it to the main portion before titration.

c. DETERMINATION OF THE TRUE VALUE FOR FERRIC IRON.

The total iron in the rock having in one way or another been found, it remains to deduct an amount equivalent to the ferrous oxide the rock contains and a further amount corresponding to the sulphides often present, in order to get what may pass for the true value for ferric iron. That this is often only an approximation appears from the difficulties due to the presence of vanadium and the generally indeterminable effect of sulphides on the ferrous-oxide determination. (See pp. 138-140.)

\section{METHODS AIMING AT THE MORE OR LESS DIRECT DETERMINATION OF}

ALUMINUM.

a. AFTER FIRST REMOVING IRON AS SULPHIDF.

Should it be desirable for any reason to effect an actual separation of aluminum, this may best be done, up to a certain point, after the pyrosulphate fusion (f, p. 88), by removal of the iron ${ }^{a}$ by ammonium sulphide in ammonium-tartrate solution, evaporation of the filtrate, ignition of the residue with sodium carbonate and nitrate, and extraction with water, whereby titanium and zirconium are left on the filter

$a$ This being first reduced to the ferrous condition by hydrogen sulphide in acid solution in order to obviate the possibility of precipitating some titanium, which otherwise is likely to happen. (Cathrein, Zeitschr. Kryst., vol. 6, 1882, p. 246, and vol. 7, 1883, p. 250. 
as sodium-salts while chromium and vanadium are carried into the filtrate as chromate and vanadate along with aluminum and phosphorus. The further separation of the last two from the chromium and vanadium is outlined under "Phosphorus" (B, p. 122). This is as far as the separation can well be carried, and the $\mathrm{Al}_{2} \mathrm{O}_{3}$ must still be found by subtracting the $\mathrm{P}_{2} \mathrm{O}_{5}$ from the combined weights of the $\mathrm{Al}_{2} \mathrm{O}_{3}$ and $\mathrm{P}_{2} \mathrm{O}_{5}$. The possibility of loss of some $\mathrm{P}_{2} \mathrm{O}_{5}$ by volatilization ${ }^{a}$ during the bisulphate fusion must be borne in mind here, for if it takes place the final weight of $\mathrm{Al}_{2} \mathrm{O}_{3}+\mathrm{P}_{2} \mathrm{O}_{5}$ will not contain all the $\mathrm{P}_{2} \mathrm{O}_{5}$.

Some writers have recommended dissolving the ignited alumina, iron oxide, etc., in hydrochloric acid, but when the precipitate has been heated over the blast, as it should be, this is very ineffective.

b. By eXtraction With a FiXed caustic aLKaLI.

A favorite practice in some countries of Europe has been to fuse the ignited precipitate containing $\mathrm{Al}_{2} \mathrm{O}_{3}, \mathrm{Fe}_{2} \mathrm{O}_{3}, \mathrm{TiO}_{2}, \mathrm{P}_{2} \mathrm{O}_{5}$, etc. - or that of the $\mathrm{Al}_{2} \mathrm{O}_{3}, \mathrm{TiO}_{2}, \mathrm{P}_{2} \mathrm{O}_{5}$, etc., after separation of iron by ammonium sulphide in tartrate solution-with sodium hydroxide in a silver crucible, or to boil the freshly precipitated mixture with a solution of the alkali, on the assumption that the oxide of titanium is hereby rendered wholly insoluble and thus separated from the alumina. This, however, is in part an error long since pointed out by F. A. Gooch; ${ }^{b}$ who showed that pure titanic oxide is markedly soluble under both conditions of treatment. Experiments made by myself to test the extent of this error brought out the following interesting results:

When 0.045 gram of titanic oxide was fused by itself with sodium hydroxide, the clear aqueous extract of the fusion held $0.0031 \mathrm{TiO}_{2}$, or about 7 per cent, determined colorimetrically. When freshly precipitated and boiled with the alkali the solubility was less. When fused with sodium carbonate but an infinitesimal trace was dissolved, which required strong concentration for its detection. When mixed with a large excess of alumina and fused with the caustic alkali, the solubility was still very marked, though less than when alumina was absent. With a large excess of ferric oxide, with or without alumina, no titanium could be detected in the unconcentrated filtrate.

It thus appears that fusion with caustic alkali after first removing iron involves an error in the gravimetric determination of both aluminum and titanium which does not appear if the iron has not been removed.

\footnotetext{
a H. Rose speaks of such loss when volatilizing sulphuric acid in presence of phosphoric acid (Handb. f. quant. Anal., Finkener ed., vol. 2,1871, p. 575 , and elsewhere), and the fact of such loss to a very marked extent has been confirmed in this laboratory.

$b$ Proc. Am. Acad. Arts Sci., vol. 12, 1885, p. 436; Bull. U. S. Geol. Survey No. 27, 1886, pp. 16, 17.
} 
c. COMBINATION METHOD AFTER DITTRICH.

Evidently with a view to eliminating the sources of error inherent in the methods outlined in a and b above, Doctor Dittrich has evolved a procedure which is a combination of both and includes methods of determining titanium and zirconium. Since it involves considerably more work than that which has just been described in detail and has few compensating advantages beyond the colorimetric determination of titanium in absence of iron, it will be sufficient to refer the reader to it. ${ }^{a}$

d. DIRECT PRECIPITATION OF ALUMINUM BY PHENYLHYDRAZINE.

A recent and promising method for the "direct determination of alumina in presence of iron, manganese, calcium, and magnesium" is that of Hess and Campbell, ${ }^{b}$ but, as with the methods just considered, it involves finally weighing aluminum, phosphorus, and titanium together. Precipitation is made by phenylhydrazine, after first neutralizing the (preferably chloride) solution by ammonia and reducing iron by a saturated solution of ammonium bisulphite. Phenylhydrazine "precipitates aluminum from its solutions quantitatively as the hydroxide without a trace of the precipitate being redissolved in excess of the precipitant."

Dr. E. T. Allen has investigated the method in this laboratory ${ }^{c}$ and confirmed the above authors' statements in the main, finding, however, that two precipitations are needed to free the precipitate entirely from iron. "The method is excellently adapted to the separation of very small quantities of aluminum, such as a milligram or even less, from a large excess of iron, a point of considerable practical importance."

Although such conditions will not be met with in ordinary rock analysis, it will not be out of place here to give the treatment as slightly modified by Doctor Allen:

The volume of the solution may vary, according to the quantity of alumina to be precipitated, from 100 to $200 \mathrm{~cm}^{3}$. It should be heated and reduced by adding saturated ammonium bisulphite. From 5 to 20 drops, according to the quantity of iron, may be used. If the solution turns deep red (ferric sulphite), it is not acid enough, and a few drops of hydrochloric acid should be added, for the sulphite itself does not reduce ferric salts, at least not with rapidity. Now quickly bring to neutrality with ammonia, and then add several drops of dilute hydrochloric acid. If this last operation is done too slowly the oxygen of the air helps to form a little ferric hydroxide which does not always readily dissolve in the dilute acid. Finally, add from 1 to $3 \mathrm{~cm} .^{3}$ of phenylhydrazine, $d$ according to the weight of the alumina to be precipitated. If too little has been used, a few drops added to the filtrate will disclose the mistake. Stir until the precipitate has

$a$ Dittrich, M., Anleitung zur Gesteinsanalyse, 1905, pp. 9-31.

$b$ J Jour. Am. Chem. Soc., vol. 21, 1899, p. 776; Chem. News, vol. 81, 1900, p. 158.

$c$ Jour. Am. Chem. Soc., vol. 25, 1903, p. 421.

$d$ The reagent should, of course, be free from inorganic impurities which could disturb the results. The author [Allen] found one sample which after persistently giving high results was proved to contain tin, which had probably been used in its preparation. 
become sufficiently flaky and allow to settle. The supernatant liquid will now be plainly acid to litmus. One need not be disturbed if the precipitate has a brownish color, for it is not due to ferric hydroxide, but to the coloring matter contained by all phenylhydrazine which has not been freshly distilled. When the determinations are allowed to stand too long the air increases this oxidation product, and a brown insoluble scum forms on the surface of the liquid and on the sides of the vessel, which is rather troublesome to the analyst. Fortunately equilibrium appears to be established in a short time. The vessels need not stand more than an hour, at any rate. The precipitate is washed by a solution of phenylhydrazine sulphite made by adding cold saturated sulphurous acid to a little phenylhydrazine until the crystalline sulphite first formed dissolves in the excess. The solution has an acid reaction. Five to $10 \mathrm{~cm}^{.}{ }^{3}$ of this are used in $100 \mathrm{~cm} .{ }^{3}$ of hot water.

9. MANGANESE, NICKEL, COBALT, COPPER, AND ZINC.

\section{A. DIFFICULTIES IN THE WAY OF A CORRECT GRAVIMETRIC DETERMI- NATION OF MANGANESE.}

The gravimetric determination of manganese in small amounts seems to be more of a stumbling block to the average chemist than that of almost any other of the frequently occurring elements met with in mineral analysis. This is due almost always to incomplete prior separation of elements which later suffer coprecipitation with the manganese. The error is therefore generally a plus one, and often amounts to many times the weight of the manganese actually present. The importance of thorough separation of the constituents treated of in the foregoing pages is therefore manifest, particularly since to the inexperienced analyst very small amounts of manganese precipitated in the form of a higher hydroxide may easily mask a good deal of alumina or other colorless precipitates. ${ }^{a}$

\footnotetext{
$a$ It is for these reasons that Washington deems it better for the novice in rock analysis to make no attempt to determine the manganese, kut to allow the error from this neglect to distribute itself over the alumina, lime, and magnesia. The relative order of this distribution has been determined by George Steiger in the Survey laboratory recently, while analyzing a series of carbonate rocks, some of them highly siliceous. The results are instructive. Double precipitations were the rule, and iron and aluminum were thrown out by ammonia.
}

Tests showing distribution of manganese in gravimetric separations.

\begin{tabular}{|c|c|c|c|c|c|c|c|}
\hline \multirow{3}{*}{ No. } & \multicolumn{3}{|c|}{ Partial composition of rock. } & \multicolumn{4}{|c|}{ MnO by colorimetry. } \\
\hline & \multirow[b]{2}{*}{$\begin{array}{l}\mathrm{Al}_{2} \mathrm{O}_{3} \\
\mathrm{Fe}_{2} \mathrm{O}_{3}\end{array}$} & \multirow[b]{2}{*}{$\mathrm{CaO}$. } & \multirow[b]{2}{*}{ MgO. } & \multirow[b]{2}{*}{ Total. } & \multicolumn{3}{|c|}{ With- } \\
\hline & & & & & $\begin{array}{c}\mathrm{Al}_{2} \mathrm{O}_{3}, \\
\mathrm{Fe}_{2} \mathrm{O}_{3} \text { (by } \\
\text { difference). }\end{array}$ & $\mathrm{CaO}$. & $\mathrm{Mg}_{2} \mathrm{P}_{2} \mathrm{O}_{i}$ \\
\hline 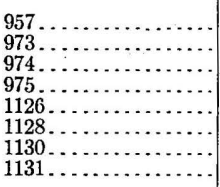 & $\begin{array}{r}2.03 \\
9.35 \\
4.80 \\
12.71 \\
.58 \\
.98 \\
3.49 \\
1.00\end{array}$ & \begin{tabular}{r|}
10.60 \\
11.84 \\
50.51 \\
11.98 \\
30.54 \\
29.69 \\
3.99 \\
28.04
\end{tabular} & $\begin{array}{r}6.30 \\
2.81 \\
1.04 \\
4.30 \\
20.41 \\
19.07 \\
.92 \\
19.11\end{array}$ & $\begin{array}{r}0.193 \\
.311 \\
.700 \\
.442 \\
.281 \\
.245 \\
.016 \\
.574\end{array}$ & $\begin{array}{l}0.085 \\
.036 \\
.301 \\
.088 \\
.030 \\
.019 \\
.016 \\
.032\end{array}$ & $\begin{array}{r}0.011 \\
.023 \\
.087 \\
.016 \\
.030 \\
.055 \\
\text { None. } \\
.101\end{array}$ & $\begin{array}{r}0.097 \\
.252 \\
.312 \\
.338 \\
.221 \\
.171 \\
\text { None. } \\
.441\end{array}$ \\
\hline
\end{tabular}

The results in 974 and 1130 particularly show how the manganese once precipitated tends to femain vith the alumina, presumably by reason of its having become peroxidized. 
If the directions already given have been carefully followed, however, there will be little chance of error due to foreign contamination, either by alumina or magnesia, a sufficiency of ammonium salts being a guaranty against the latter. Regard must be had, however, to the rather remote possibility of the presence of rare earths which were not thrown out by the basic-acetate precipitation (see third footnote, p. 83), for they will appear at this stage.

\section{B. PRECIPITATION OF THE GROUP AND SEPARATION OF ITS CONSTITU-} ENTS.

a. THE AMMONIUM-SULPHIDE METHOD.

$\alpha$. Its advantages and disadvantages.-On the other hand the usual methods of separation of manganese from the alkaline earths and magnesia by bromine or ammonium sulphide are imperfect, in part by reason of incompleteness, in part because of coprecipitation of small amounts of these metals. The former error, tho absolutely slight, is in rock analysis probably of more consequence than the latter. In regard to completeness of precipitation bromine offers no advantage over ammonium sulphide, and the latter has the advantage that by a single operation nickel, cobalt, copper, and zinc, if present, are likewise separated from the earth metals and alkalies. There need be no feãr of overlooking nickel or copper, for under the conditions of the precipitation they are not retained in solution. It is for this reason chiefly that the ammonium-sulphide method is to be preferred. Most of the small amount of manganese that escapes precipitation is weighed later with the magnesium as pyrophosphate and can be readily corrected for by the colorimetric method described on page 99 .

The precipitation of manganese in alkaline solution by hydrogen peroxide, as proposed by P. Jannasch and E. v. Cloedt, ${ }^{a}$ a method which appeared to be simple and accurate, besides affording a separation from zinc, has been shown by C. Friedheim and $\mathrm{E}$. Brühl ${ }^{b}$ to be valueless.

$\beta$. Precipitation by ammonium sulphide and separation of manganese and zinc from nickel, cobalt, and copper.-Two or three $\mathrm{cm} .^{3}$ of ammonia are added to the flask containing manganese, the earth metals, etc. (8. A. d. $\alpha, \beta$, pp. 85,86 ), and hydrogen sulphide gas is introduced to saturation, whereby manganese, nickel, cobalt, copper, zinc, and a small part of the platinum from the dish are precipitated. A second like amount of ammonia is now added. The flask, filled to the neck and corked, is set aside for at least twelve hours, and prefer- 
ably twenty-four, or even longer. The precipitate, collected and washed on a small filter with water containing ammonium chloride and sulphide, is extracted by hydrogen-sulphide water acidified with one-fifth its volume of hydrochloric acid (sp.gr. 1.11), manganese and zinc, if present, going into solution.

(For treatment of the ammonium-sulphide filtrate see 10, p. 101.)

b. MANGANESE AND ZINC.

The hydrochloric-acid filtrate is evaporated to dryness, ammonium salts are destroyed by evaporation with a few drops of sodium-carbonate solution, hydrochloric acid and a drop of sulphurous acid are added to decompose excess of carbonate and to dissolve precipitated manganese, and the latter is reprecipitated at boiling heat by sodium carbonate after evaporation of the hydrochloric acid. If zinc is present, it can be separated from the manganese after weighing. For the small quantities of manganese usually found the sodium-carbonate method of precipitation is to be preferred to that by bromine or sodium phosphate, as equally accurate and a time saver.

The manganese is weighed as $\mathrm{Mn}_{3} \mathrm{O}_{4}$ and calculated to $\mathrm{MnO}$, or, if present in some quantity, it may preferably be weighed as the sulphate, ${ }^{a}$ or, finally, it may be determined colorimetrically with much greater certainty, as in C, page 99, when the amount is within the usual limits for rocks. In the last case, in the absence of zinc, the hydrochloric-acid solution of the sulphide may be used for the colorimetric test, after first evaporating off the hydrochloric acid with nitric or sulphuric acid.

\section{NICKEL, COBALT, COPPER.}

The paper containing nickel, cobalt, and copper is incinerated in porcelain, dissolved in a few drops of aqua regia, and evaporated with hydrochloric acid; the copper and platinum are thrown out warm by hydrogen sulphide, and in the filtrate made ammoniacal nickel and cobalt are thrown down by hydrogen sulphide. This liquid is then rendered faintly acid by acetic acid and allowed to stand. The sulphide of nickel after filtering is simply burned and weighed as oxide-its weight being always very small, hardly ever over 0.3 or 0.4 milligram - and is then tested for cobalt in the borax bead. The color of the last drop or two of the hydrochloric-acid solution, after decomposing the aqua regia, is a good indication of the presence or absence of nickel and cobalt. It is always well at this point to separate by ammonia any traces of iron that may be present, then to acidify and treat with hydrogen sulphide as above.

$a$ Volhard, J., Ann. Chem., vol. 198, 1879, p. 329. Gooch, F. A., and Austin, Martha, Am. Jour. Sci., 4 th ser., vol. 5, 1898, p. 209; Zeitschr. anorg. Chemie, vol. 17, 1898, p. 264. 
It is somewhat unsafe to consider traces of copper found at this stage to belong to the rock if the evaporations have been conducted, as is usually the case, on a copper water or steam bath, or if water has been used which has been boiled in a copper kettle, even if tinned inside. Therefore, and because of its contamination by a little platinum, it is better to determine copper in a separate portion if its presence is indicated with certainty. (See 7, p. 81.)

\section{COLORIMETRIC DETERMINATION OF MANGANESE.}

a. PRELIMINARY TREATMENT.

As has been shown above (p. 96), the gravimetric determination of manganese is subject to grave error, even when great care is used. This is due to a variety of reasons, such as incompleteness of separation from aluminum and iron, incomplete precipitation by ammonium sulphide, and contamination by other bodies. Because of the small amounts in question these errors may be relatively enormous, and they can not be depended on to balance each other. Therefore, it would be an advance if the total manganese could be determined in a separate portion of the sample, and in the main portion only that part which is weighed with the magnesium pyrophosphate, and this only as a correction to the magnesia. This course is readily feasible with carbonate rocks, which can usually be brought into a fit state in a few minutes without a fusion or the separation of silica. With silicate rocks more labor is involved, but the determination itself is very accurately performed by colorimetry, as in the case of carbonates.

The preliminary treatment consists in decomposing a gram or half a gram of the rock powder in a small platinum dish or capacious crucible (placed in the radiator shown in fig. 2, p. 29) with hydrofluoric and sulphuric acids till all is disintegrated. The hydrofluoric acid is then to be driven off by repeated evaporations with small portions of sulphuric acid, after which nitric acid free from chlorine is added, together with water, and as much of the residue is brought into solution as possible. What remains insoluble is usually barium and calcium sulphates. This is filtered off on a small filter, the filtrate is caught in a small beaker or flask, and the manganese determined as in the following paragraphs.

b. COLORIMETRIO DETERMINATION OF MANGANESE BY AMMONIUM PERSULPHATE. $a$

This method has the advantage over that by lead peroxide in that, unless chlorides happen to be in the solution, no filtration is necessary. Two solutions are needed, one of silver nitrate containing 2 grams of the salt to the liter, and one of manganous sulphate or nitrate

$a$ Walters, H. E., Chem. News, vol. 84, 1901, p. 239; Proc. Eng. Soc. West. Pa.., vol. 17, 1901, p. 257. 
containing the equivalent of 2 milligrams of $\mathrm{MnO}$ in $10 \mathrm{~cm} .^{3}$. This last can be conveniently made by acidifying a standard permanganate solution, reducing it by sulphurous acid, and diluting appropriately. A solution of permanganate can be used directly as a color standard when very small quantities of manganese are in question. Ammonium persulphate in the solid form is also needed. The commercial article needs no purification ordinarily in this case.

The rock solution must be strongly acid with nitric or sulphuric acid and considerably less than $100 \mathrm{~cm}^{3}$ in bulk unless the manganese exceeds 1 milligram in weight. To it is added $10 \mathrm{~cm}^{3}$ of the silver solution for every milligram of metallic manganese, and it is transferred to a graduated flask of size suited to the solution. Should it show a turbidity from precipitated silver chloride, the liquid must be agitated and then filtered into the flask. There is now added about 1 gram of solid ammonium persulphate and the flask is placed on the steam bath or hot plate. Very soon the pink color of permanganic acid begins to appear and rapidly increases to a maximum. The flask may be removed soon after the color begins to show and placed in cold water when it is fully developed. If the depth of color is very great the solution must be poured into a larger flask. The flask is filled with water to the mark and the contents thoroughly mixed.

According to the depth of color there is now placed in another flask of $100 \mathrm{~cm} .^{3}$ or less volume $10 \mathrm{~cm} .^{3}$, or some multiple of that amount, of the manganese solution, so that when both solutions are oxidized and the flasks filled the color in the standard shall be more intense than in the test solution. This solution is oxidized by the aid of silver salt and persulphate in the same manner as the other, cooled, filled to the mark, shaken, and poured into a burette. Ten $\mathrm{cm}^{3}$ is drawn off into one of the comparator cylinders shown in fig. 5 (p. 32), and diluted with water from a burette till the color equals that of the properly diluted and mixed test solution, a part or all of which has been poured into the companion cylinder. The observations are made exactly as given on page 32 . If the form of colorimeter depicted in fig. 6 is used, the dilution of the standard solution is, of course, unnecessary. Nessler cylinders can be used instead of either of the colorimeters described. The results are very exact.

Should, perchance, a brown precipitate (due to deficiency of silver salt) have formed during the oxidation of the standard, it is best to start with a fresh portion, but in case this happens with the test solution the precipitate must be brought into solution by a little sulphurous acid, more silver salt added, and the oxidation repeated, if need be, after adding more persulphate. 


\section{CALCIUM AND STRONTIUM (BARIUM).}

\section{A. SEPARATION FROM MAGNESIUM.}

a. PRECIPITATION AND IGNITION OF CALCIUM AND STRONTIUM OXALATES TOGETHER.

The platinum derived from the dish in the silica evaporation, except for the small portion precipitated with the manganese sulphide, is now wholly in the filtrate from the latter (9. B. a. $\beta$, p. 97). Its separation at this or any other stage is quite unnecessary; nor is the removal of ammonium chloride usually demanded, since there is no undue amount present in most cases, the first precipitation of alumina, etc., having been by ammonium or sodium acetate. ${ }^{a}$ Therefore, without destroying ammonium sulphide the calcium and strontium are thrown out by ammonium oxalate at boiling heat, the precipitate, often darkened by deposited platinum sulphide, is ignited and redissolved in hydrochloric acid, boiled with ammonia to throw out traces of alumina sometimes present, and reprecipitated as before, but in a small bulk of solution. It is weighed as oxide, transferred to a small flask of 20 $\mathrm{cm} .^{3}$ capacity, dissolved in nitric acid, evaporated to dryness at $150^{\circ}$ to $160^{\circ}$, and the separation of strontium from calcium effected by ether-alcohol $^{b}$ as described below (B, p. 102).

The weight of strontia found deducted from that of the two oxides gives that of the lime. For treatment of the filtrates see "Magnesium" (11, p. 105).

b. NOtes on DOUble precipitation, Washing, and ignition of the oxalates.

It may be said with regard to the separation of calcium from magnesium that two precipitations by ammonium oxalate are essential to the attainment of correct results, not only for the complete removal of magnesium but of sodium as well, the retention of compounds of the latter element by calcium oxalate being now generally known. This subject has been thoroughly studied by T. W. Richards, C. T. McCaffrey, and $\mathrm{H}$. Bisbee. ${ }^{c}$ They also call attention to the danger of too long washing with hot water because of the very appreciable solubility of calcium oxalate. This is a point that needs greater attention than it ordinarily receives from analysts.

The ignition is started with the precipitate moist. After charring and burning off the paper the crucible is to be covered and blasted with an inclined flame. It is a very mistaken idea that with a really good blast it takes long to reach constant weight. Five minutes is ordinarily ample for any amount of lime that is likely to be met

\footnotetext{
$a$ If two or three precipitations by ammonia alone are depended on, the second and third filtrates are evaporated rapidly to dryness and the ammonium salts removed by ignition.

$b$ See R. Fresenius, Zeitschr. anal. Chemie, vol. 32,1893 , pp. 189, 312, for the latest improvements in this method, in their application to considerable quantities.

c Proc. Am. Acad. Arts Sci., vol. 36, 1901, p. 375; Zeitschr. anorg. Chemie, vol. 28, 1901, p. 71.
} 
with. For ordinary amounts the heat of a large and good Bunsen burner is in reality sufficient, since I was able by it to reduce 3 grams of calcium carbonate in fifty minutes to the oxide, or so nearly so that the blast afterwards caused a further loss of only 1 milligram. This, of course, was with the crucible covered. The lime should not be left over half an hour in the desiccator before weighing, for it gains weight appreciably if left long.

\section{B. SEPARATION OF STRONTIUM (BARIUM) FROM CALCIUM.}

a. BY ETHER-ALCOHOL.

The thoroughly dried nitrates obtained in A. a are treated with as little (rarely over $2 \mathrm{~cm}^{3}$ ) of a mixture in equal parts of absolute alcohol and ether as may be needed to dissolve the calcium salt, solution being. hastened by occasional gentle agitation. After standing overnight in the corked flask the insoluble matter is collected on the smallest possible filter and washed with more of the above mixture of alcohol and ether. After drying, a few cubic centimeters of hot water are passed through the filter, on which may remain a few tenths of a milligram of residue, which does not usually contain any lime or other alkaline earth and whose weight is therefore to be deducted from that of the lime, unless it can be shown that it is derived from the glass of the little flask in which the nitrates of calcium and strontium were evaporated. To the solution of strontium nitrate in a small beaker a few drops of sulphuric acid and then its volume of alcohol are added, whereby the strontium is precipitated as sulphate, in which form after twelve hours it is weighed and then tested spectroscopically as to freedom from calcium and barium.

$$
\text { b. BY AMYL ALCOHOL. }
$$

Because of the slight solubility of strontium nitrate in amyl alcohol the method of Browning ${ }^{a}$ does not appear to be adapted to the separation from calcium of the small amounts of strontium met with in rocks, though with barium the case is different, since its nitrate according to Browning is insoluble in absolute amyl alcohol.

\section{BEHAVIOR OF BARIUM.}

Barium, after two ammonium-oxalate precipitations, will never be found with the ignited calcium and strontium in more than spectroscopic traces, unless originally present in excess of 3 or 4 milligrams; and very often only when in considerable excess. ${ }^{b}$ If present with them, however, it will be separated with the strontium by ether-

a Am. Jour. Sci., 3d ser., vol. 43, 1892, pp. 50, 314 .

$b$ Hillebrand, W. F., Jour. Am. Chem. Soc., vol. 16, 1894, p. 83; Chem. News, vol. 69, 1894, p. 147. 
alcohol or amyl alcohol, and these two must then be treated by the ammonium-chromate method, given below, in order to arrive at the strontium. The barium is best estimated in a separate portion. (See "Barium"13, p. 116.)

\section{SEPARATION OF BARIUM FROM STRONTIUM.}

Profiting by the experience of R. Fresenius and others, A. Skrabal and L. Neustadl ${ }^{a}$ have shown in what manner alone a very nearly correct separation of barium can be made from calcium and strontium. The following solutions are requisite:

Ammonium bichromate, 100 grams to the liter.

Ammonium acetate, $a, 300$ grams, neutralized by ammonia, to the liter.

Ammonium acetate, $b, 20 \mathrm{~cm} .{ }^{3}$ of $a$ diluted to 1 liter.

The reaction of the acetate solutions should be alkaline rather than acid.

The method for the amounts used by the authors (0.135 gram $\mathrm{BaO}$ as the chloride and the same of strontium) is as follows:

To the neutral or weakly acid solution is added ammonium acetate (10 $\mathrm{cm} .^{3}$ of solution $a$ ) in excess. The liquid is brought to boiling and while swirled about $5 \mathrm{~cm}^{3}$ of the bichromate solution is added. After settling and cooling the clear liquid is decanted through a filter and the precipitate washed by decantation with ammonium acetate (solution $b$ ) till the filtrate is no longer perceptibly colored $\left(100 \mathrm{~cm}^{3}\right.$ of wash solution). The beaker is placed under the funnel, the precipitate on the paper dissolved by warm dilute nitric acid, and the paper washed. More acid is then added to dissolve the rest of the precipitate, followed by ammonia till the precipitate forming again no longer redissolves. Ammonium acetate $\left(10 \mathrm{~cm} .^{3}\right.$ of solution $\left.a\right)$ is now poured in, the liquid brought to boiling while kept in swirling motion, allowed to cool slowly, and the precipitate is washed by decantation with solution $b$ of the ammonium acetate. The barium chromate is dried and weighed as such after ignition, the filter being burned separately.

The strontium may be thrown down from the combined filtrates by ammonia and ammonium carbonate, after concentration in presence of a little nitric acid, and weighed as carbonate; or the carbonate may be redissolved, precipitated by sulphuric acid and alcohol, and weighed as sulphate.

It is probable that for the small amounts encountered in rocks a single precipitation of the barium by bichromate will suffice, but this is not so when any considerable quantities are to be separated. 
E. OTHER METHODS OF DETERMINING THE WEIGHT OF CALCIUM.

GENERAL STATEMENT.

Additional methods more or less in vogue for determining the calcium after precipitation as oxalate are the volumetric method by potassium permanganate and the conversion of the oxalate into carbonate, sulphate, or fluoride. None of these is applicable in rock analysis if it is desired to determine the strontium, except the second, but any one of them will apparently afford good results if no account is to be taken of that element. The methods have, however, not been tested in this laboratory, though the several gravimetric methods have been compared by O. Brunck, ${ }^{a}$ who finds that duplicates by any one of them show better agreement than by the oxide method. Brief descriptions of them are therefore given below, as well as of the volumetric method.

It is to be said, however, regarding Brunck's determination of calcium as the oxide that no such variations as those reported by him are ever obtained in the Survey laboratory. Our duplicates by that method agree as well as his by the other methods. His high and varying results by the oxide method are probably to be attributed to the employment of a vertical instead of an inclined flame. The complete conversion of the oxide to sulphate by treatment with either sulphuric or hydrofluoric acid is, in my opinion, based on experience with the conversion of lead chloride to sulphate, by no means easily brought about. There will always be a tendency, where an insoluble compound is at once produced by action of one of the acids in question, for some of the original compound to be protected from conversion to the salt, even after two or more evaporations with fresh portions of acid.

It is further doubtful if the loss in weight of the crucible during blast ignition of the lime is really as great as he thinks. It is well known that barium oxide attacks platinum strongly when heated in contact with it, and it is certain that lime does so to a less but appreciable extent. That it does can readily be demonstrated by dissolving in hydrochloric acid lime that has been blasted in platinum for half an hour and precipitating and weighing the dissolved platinum. The amount recovered will nearly and sometimes quite equal the loss in weight of the crucible itself.

a. VOLUMETRIO METHOD.

The washed oxalate is rinsed into a beaker and the filter is extracted with hot dilute sulphuric acid, the filtrate being caught in the beaker. It is not permissible to omit this extraction, for simple rinsing with water will not extract all of the oxalate held in the pores of the paper. 
More acid is added if necessary, and the contents of the beaker are digested at a moderate heat till decomposition can be considered complete. The oxalic acid is then titrated warm at about $70^{\circ}$ with permanganate, which has been standardized best against pure Iceland spar dissolved in hydrochloric acid and precipitated as the oxalate.

\section{b. BY WEIGHING AS THE CARBONATE.}

The oxalate is gently ignited to convert it to carbonate and is then digested with a few drops of a solution of ammonium carbonate. The liquid is evaporated cautiously and the residue gently ignited to just visible dull redness and weighed. The treatment with ammonium carbonate, etc., is repeated till constant weight is reached.

c. BY WEIGHING AS THE SUlPhate.

The strongly ignited oxalate is cautiously slacked with water and a slight excess of sulphuric acid is added. The liquid is concentrated by evaporation and the excess of acid removed in the radiator (fig. 2, p. 29). The dry sulphate is then moderately ignited (to dull redness for a short time) and weighed, the treatment with acid, etc., being repeated to constant weight.

d. BY WEIGHING AS THE FLUORIDE.

The strongly ignited oxide is slacked with water and covered with hydrofluoric acid, the excess of which is removed on the bath and the residue ignited for a few moments and weighed. The treatment with acid, etc., is to be repeated till the weight becomes constant. According to Brunck this last method is simpler than the carbonate or sulphate method, and in the hands of the inexperienced leads to better results, though he regards the sulphate method as the best in experienced hands.

\section{MAGNESIUM.}

\section{A. PRecipitation.}

a. CONDITIONS NECESSARY FOR THE PRODUCTION OF A NORMAL PRECIPITATE.

There are few analytical procedures regarding which so much uncertainty has existed and still exists as that by which magnesium is precipitated as the ammonio-phosphate, notwithstanding the enormous mass of detailed work that has been done on the subject. - The work of H. Neubauer, ${ }^{a}$ confirmed in part and supplemented by F. A. Gooch and Martha Austin, ${ }^{b}$ seemed to clear the ground fairly well. Neu-

\footnotetext{
a Zeitschr. angew. Chemie, 1896, p. 435.

${ }^{b}$ Am. Jour. Sci., 4th ser., vol. 7, 1899, p. 187; Chem. News, vol. 79, 1899, pp. 233, 244, and 255; Zeitschr. anorg. Chemie, vol. 20, 1899, p. 121.
} 
bauer found that precipitation is complete even in the presence of large quantities of salts of ammonium, including the oxalate, but that the composition of the precipitate is largely affected by ammonium salts and also by the way in which the precipitation is made. Gooch and Austin show that the large amount of ammonia of 0.96 specific gravity (one-third the original volume of the solution) usually prescribed is not only unnecessary, but, in fact, disadvantageous, at least, until the precipitate has fully formed. The authors named agree that it is only by working under certain conditions-absence of any large excess of precipitant, of ammoniacal salts, and of ammonia - that a precipitate of normal composition is obtainable (see below). From their labors it would seem clear that the common way of adding the phosphate precipitant to the ammoniacal solution of the magnesium salt is not calculated to produce a precipitate of normal composition. The precipitant should be added to the acid solution of the magnesium, and ammonia should then be added in slight excess.

The precipitate as ordinarily formed differs from the normal in containing relatively more ammonium and less magnesium-for instance, an admixture of such a molecule as $\mathrm{Mg}\left(\mathrm{NH}_{4}\right)_{4}\left(\mathrm{PO}_{4}\right)_{2}$-the result being that when ignited in the ordinary way too much magnesium is found because of formation of some metaphosphate. To obviate this error, Neubauer considers it absolutely necessary to blast the precipitatefor half an hour and then to repeat the blasting for a second half hour to see if constant weight has been reached. The phosphate is then entirely pyrophosphate, which is quite unaffected by further blasting (see, however, B. a, p. 108). The intense heat has caused a decomposition of the metaphosphate with volatilization of $\mathrm{P}_{2} \mathrm{O}_{5}$, as follows: $2 \mathrm{Mg}\left(\mathrm{PO}_{3}\right)_{2}=\mathrm{Mg}_{2} \mathrm{P}_{2} \mathrm{O}_{7}+\mathrm{P}_{2} \mathrm{O}_{5}$. Neubauer worked with the usual excess of ammonia added after the bulk of the precipitate had formed, and it remains to be seen whether by precipitating and working according to Gooch and Austin the composition of the precipitate is always close enough to the ideal $\mathrm{MgNH}_{4} \mathrm{PO}_{4}$ to obviate the necessity for blasting.

Gooch and Austin call attention to a modification proposed long since by Wolcott Gibbs, ${ }^{a}$ whereby the phosphorus and magnesium salts are first boiled together in neutral solution for a few minutes and to the cooled solution ammonia is added. The results are said to be remarkably exact, but K. K. Järvinen ${ }^{b}$ reports to the contrary. This chemist obtained a normal precipitate by either of two methods of his own, of which he regards the following as more convenient and reliable, because ammonium salts exert no influence on the result. It is likewise applicable to the precipitation of phosphoric acid by magnesia mixture. 
The hot magnesium solution, containing at least 2 to 3 grams of ammonium salts (to prevent precipitation of magnesium hydroxide) and 20 to $30 \mathrm{~cm}^{3}$ of 10 per cent ammonia solution for 0.4 gram $\mathrm{Mg}_{2} \mathrm{P}_{2} \mathrm{O}_{7}$, is heated to boiling and the precipitant is added slowly. Then more ammonia is added and the solution allowed to cool. A. coarsely crystalline precipitate forms which, it is claimed, consists entirely of dimagnesium-ammonium phosphate. This procedure of Järvinen was employed where but a single precipitation of themagnesium was intended, and it does not seem to be adapted for a second precipitation. As this is always called for in rock analysis, it does not appear best to adopt his procedure for our purposes.

b. PRECIPITATION.

$\alpha$. First precipitation.-This is made without special precautions in the filtrate from the first calcium-oxalate separation (p. 101) by sodiumammonium-hydrogen phosphate (microcosmic salt) ${ }^{a}$ in indefinite decided excess and without the great excess of ammonia usually prescribed. It is not necessary to first remove ammoniacal salts unless very little magnesium is present, and then only in order to hasten precipitation. The facts that large quantities of these salts retard precipitation and that the composition of the precipitate is affected by them as well as by the way in which the precipitation is made are of importance only when a single precipitation is to be made or in the final of two or more.

Continued agitation of the liquid by a mechanical stirrer is of advantage in greatly shortening the time of complete precipitation, which is in general shorter the greater the quantity of magnesium present.

$\beta$. Second precipitation.-Platinum sulphide usually strongly contaminates the separated phosphate, but this matters not, as it remains on the filter when the phosphate is redissolved in hydrochloric acid, of which not more than the amount really needed should be used. The solution thus obtained is united with that of the residue from evaporation and ignition of the second filtrate from the calcium oxalate (10. A. a, p. 101), and is diluted if necessary. A few drops of sodium-ammonium-phosphate solution are now added, and ammonia, drop by drop, in slight excess, with constant stirring till the crystalline precipitate has well formed. Once formed a few cubic centimeters more of ammonia are to be added.

$a$ The objection that has been made by one writer to the use of this salt instead of disodium-hydrogen phosphate is, so far as our experience teaches, entirely groundless. 


\section{B. Methods OF COLLECTING AND IGNITING THE PRECIPITAte.}

a. ORDINARY PROCEDURES.

Were it not for the necessity of examining the precipitate for impurities after ignition (C., p. 109), it were best collected in a Gooch crucible, provided the asbestos felt is well constructed and not of the serpentine variety so largely on the market. It is therefore well to use an ordinary crucible of platinum. Neubauer ignites slowly in platinum after drying, without removing from the paper, and applies the bdast only when the carbon has been wholly burned off. ${ }^{a}$ The danger of attack of the platinum by thus igniting the precipitate in contact with the paper is extremely slight, though it has happened once or twice in our experience that a crucible has been ruined by so doing. Why reduction should have occurred in these cases and not in the hundreds of others is not known.

The precipitate, wrapped in its moist paper, is best dried in the crucible itself, the paper then slowly charred without allowing it to ignite, the carbon burned off over a gradually increasing flame, and finally a weak blast applied for a long time and repeated to constant weight to insure volatilization of any excess of $\mathrm{P}_{2} \mathrm{O}_{5}$ over and above that required for the pyrophosphate formula (see A. a, p. 105).

The experience of the Survey chemists was for a time decidedly adverse to the blasting of magnesium pyrophosphate as prescribed by Neubauer, whether obtained by precipitation of magnesium or of phosphate solutions. If the weights were not large the salt fused in part or wholly, and when this occurred reduction seemed to be rapid and the crucible was rather strongly attacked, as shown by its loss in weight after dissolving out the pyrophosphate. Presumably this effect was caused by a blast of higher temperature than that employed by Neubauer, who is very emphatic in his assertion that neither pure pyrophosphate nor crucible.suffers the slightest loss when blasted for hours. This seems to be true with a blast of but moderate temperature, giving less than $1,100^{\circ}$ in the crucible. What effect the presence of slight amounts of foreign phosphates may have in increasing the fusibility of magnesium pyrophosphate is unknown. Järvinen found that his precipitates obtained by the Gibbs method fused readily and gave too high results, and further that $\mathrm{P}_{2} \mathrm{O}_{5}$ did seem to volatilize slowly from $\mathrm{Mg}_{2} \mathrm{P}_{2} \mathrm{O}_{7}$ over the blast.

\section{b. MODIFICATION OF ULBRICHT.}

At one time the procedure first recommended by $\mathrm{R}$. Ulbricht, later by K. Broockmann, and also by L. L. de Koninck, was used. It consists in dissolving the ammonium-magnesium phosphate off the filter 
with nitric acid, collecting the filtrate in a weighed crucible, evaporating the contents to dryness, ${ }^{a}$ and subsequently igniting, the product being presumably pyrophosphate. But it was soon observed that the ignited salt, especially when large in amount, does not always dissolve completely in hydrochloric acid, but that sometimes a white residue is left in light lumps which appears to be quite insoluble in acids. This residue contains no silica, but only the constituents of a magnesian phosphate, and it may be a peculiar metaphosphate. Whether its appearance is due to an abnormal composition of the original magnesian precipitate or to conceivable change during evaporation in the crucible with nitric acid remains to be determined. Until this is done the employment of this method of igniting is not to be recommended.

\section{CONTAMINATION BY AND REMOVAL OF BARIUM, CALCIUM, AND} MANGANESE.

a. BARIUM.

Barium phosphate will not contaminate the second magnesian precipitate unless there are notable amounts of barium in the rock, in which case it must be removed by sulphuric acid prior to the final precipitation of the magnesium.

\section{b. CALCIUM.}

Calcium, however, is probably never absent, and has to be estimated and allowed for as follows:

To the ignited pyrophosphate, dissolved in but slight excess of hydrochloric acid, is added ammonia to alkalinity, and then acetic acid, drop by drop, till the solution, which should measure 10 to $30 \mathrm{~cm} .^{a}$ and not be hot, clears. It now and then happens that a little flocculent matter fails to dissolve. This is to be removed, ignited, and subtracted from the original weight, if proved to be free from magnesium, which it often contains (see c, p. 110). It is likely to consist, in great part or wholly, of phosphates of iron or manganese, or both, and shows often a reddish color on ignition. If an excess of acetic acid has been used, this is cautiously removed by ammonia. Then a drop or two of solution of ammonium oxalate is added, and the small beaker is set aside for twelve hours if necessary. Almost invariably a small precipitate soon shows itself, which, if fine grained and nonadherent to the glass, may be regarded as pure calcium oxalate; otherwise it contains, or may largely consist of, magnesium oxalate. It is in that case to be collected, ignited, redissolved, and reprecipitated. Its final weight, averaging perhaps one-half milligram, is to be added to that of the lime already found and subtracted as tricalcium phosphate (not pyrophosphate) from that of the magnesium pyrophosphate, in order

\footnotetext{
a A pink color of varying intensity almost invariably becomes apparent as the mass approaches dryness, a most delicate test for the traces of manganese which always escape preapitation by ammonium sulphide or bromine.
} 
to arrive at the true figure for magnesia. This separation, to be satisfactory, requires great care.

$$
\text { c. MANGANESE. }
$$

A separation from one another of the iron, aluminum, and manganese which the small flocculent precipitate mentioned in b may contain is not worth the trouble, careful prior treatment being presupposed. The first two constituents may have been introduced since the original precipitation of the iron and aluminum, and their amount in terms of those elements is very small. But the amount of manganese it contains may be a sensible proportion of the whole of that element. The precipitate should therefore be brought into nitric-acid solution. The solution containing the magnesium is also manganiferous. It is to be evaporated to dryness, the volatile salts expelled by ignition, and the residue again evaporated two or three times with a few drops of nitric acid to certainly remove all traces of chlorine, and united with the small solution above mentioned. The manganese is now determined colorimetrically, as in 9.C.b, page 99, and its amount added to that already found, unless the total has been ascertained from a separate portion of the sample.

The distribution of the manganese over the alumina, lime, and magnesia, when ammonia has been employed for the precipitation of the alumina and no attempt has been made to remove the magnanese at all, is shown in the table on page 96 .

\section{LIABILITY TO ERROR IN THE AVERAGE MAGNESIUM DETERMINATION.}

Experience has shown that the majority of analysts are pretty sure to make a very serious plus error in determining moderate amounts of magnesia, such as are found in limestones and Portland cements. The causes of this error will be sufficiently apparent after a careful perusal of the foregoing pages. Everyone ought to make the tests for foreign contamination just described, if only to convince himself of the reality of the error even after very careful work.

\section{TITANIUM.}

\section{A. COLORIMETRIC DETERMINATION WITH HYDROGEN PEROXIDE.}

a. DESCRIPTION OF THE METHOD (WELLER'S), $a$

The method consists in comparing the color of a known bulk of solution to be tested with that of a standard solution of titanium sulphate, both having been fully oxidized by hydrogen peroxide. The strength of the peroxide should be approximately measured by titration with. permanganate on opening a fresh bottle and again after a few weeks, otherwise very serious error may arise through its deterioration. 
Mere traces of hydrofluoric acid, in either the peroxide or the titanium solution, render this method inexact, ${ }^{a}$ hence care should be exercised as to the character of the peroxide, which, as sold in the market, often contains fluorine. For method of testing see page 36 .

Dunnington $^{b}$ has pointed out the necessity for the presence of at least 5 per cent of sulphuric acid in solutions which are to be thus tested for titanium, in order, as he concludes, to prevent partial reversion to metatitanic acid, which does not give a color with hydrogen peroxide. The standard solution of titanium sulphate, ${ }^{c}$ holding conveniently about 1 centigram $\mathrm{TiO}_{2}$ in $10 \mathrm{~cm}^{3}$, equivalent to 1 per cent of $\mathrm{TiO}_{2}$ in 1 gram of rock, contains, therefore, 5 per cent or more of sulphuric acid. Of this, $10 \mathrm{~cm} .^{3}$ are mixed with a sufficiency of hydrogen peroxide ( $2 \mathrm{~cm} .^{3}$ of most commercial brands is ample) and diluted to $100 \mathrm{~cm} .^{3}$ in a measuring flask.

Titanium can be determined, as a rule, most conveniently in the solution which has served for the titration of total iron ( $\gamma$, p.92). This, having been evaporated, if necessary, to less than $100 \mathrm{~cm}^{3}$, is to be fully oxidized with hydrogen peroxide, and if the color is less intense than that of the standard, is made up to $100 \mathrm{~cm} .^{3}$ with dilute sulphuric acid in a measuring flask, and mixed; otherwise, in a flask of sufficient size to insure that its color shall be less intense. One of the rectangular glasses (fig. 5, p. 32) being filled with the solution to be tested, $10 \mathrm{~cm}^{3}$ of the diluted standard are run into the other from a burette, and water is added from a second burette until there is no distinction as to color. A second and a third portion of the standard can be run in and diluted and the mean of several determinations struck, when a simple calculation gives the percentage of $\mathrm{TiO}_{2}$ in the rock, the amounts in the two solutions being directly as their volumes.

If the convenient but expensive Soleil-Duboscq colorimeter is used, or the modification of O. Schreiner (fig. 6, p. 33), or the simple Nessler tubes, it is of course unnecessary to dilute the rock solution to the extent above required, should it be stronger than the standard. Experience has shown, however, that differences can not be sharply estimated in strongly colored solutions, and that the results are much more satisfactory when the color intensity is not much if any greater than that given by a standard of the above concentration. For

$a$ Hillebrand, W. F., Jour. Am. Chem. Soc., vol. 17, 1895, p. 718; Chem. News, vol. 72, 1895, p. 158; Bull.

U. S. Geol. Survey No. 167,1900 , p. 56.

$b$ Jour. Am. Chem. Soc., vol. 13, 1891, p. 210.

$c$ Potassium-titanium fluoride, $\mathrm{K}_{2} \mathrm{Ti}_{6}$, best serves as the starting point for its preparation. A quantity of this is recrystallized from boiling water once or more times, dried, and preserved in a glass-stoppered bottle. Enough of it to make one-half to 1 liter of the standard sulphate solution is weighed into a platinum dish and evaporated several times, without bringing to dryness, with strong sulphuric acid till the fluorine is completely expelled. The residue is then taken up with water containing enough sulphuric acid to make 5 per cent of the latter when fully diluted. Two $50-100 \mathrm{~cm} .{ }^{3}$ portions of the prepared solution are then further diluted bolled, and precipitated with ammonia. The precipitates are collected on paper, washed with hot water till free from alkali, ignited moist in the filter, blasted, and weighed. Duplicates should agree almost exactly. 
the percentages of titanium found in rocks, clays, and soils, usually under 1 per cent, but rising to 2 or even 3 per cent or more occasionally, the colorimeter method gives results which are fully equal to those of the best gravimetric method, besides being a great time saver.

b. CORRECTION FOR THE COLOR OF FERRIC SULPHATE.

The error introduced by iron, in consequence of the yellowish color of its sulphate solution, is practically negligible unless its percentage is high; then either the iron must be removed, as described under B.a. $\alpha$, page 113, prior to making the color test, or correction should be applied for known amounts of ferric sulphate in solutions of the requisite dilution.

The exact correction to be applied in such cases is difficult of determination because of the impossibility of matching the colors of titanium-peroxide solutions with those of ferric sulphate; but tests made go to show that the coloring effect of 0.1 gram of $\mathrm{Fe}_{2} \mathrm{O}_{3}$ in $100 \mathrm{~cm} .^{3}$ of 5 per cent sulphuric-acid solution is about equal to 0.2 milligram of $\mathrm{TiO}_{2}$ in $100 \mathrm{~cm} .^{3}$ when oxidized by hydrogen peroxide. This amounts to a correction of only 0.02 per cent on one gram of rock containing the unusual amount of 10 per cent $\mathrm{Fe}_{2} \mathrm{O}_{3}$.

W. A. Noyes ${ }^{a}$ overcomes the difficulty mentioned in the last paragraph by adding to the standard solution approximately as much ferric iron as will give it, after the colors are matched, the same iron strength as the test solution. This could not be done with the apparatus depicted in fig. 6 (p. 33), though practicable with Nessler tubes. In using the Survey colorimeter (fig. 5 , p: 32 ), it would perhaps be best to make first an approximate determination of the titanium in order to find the final volume of the standard after dilution, then to make an exact determination, having added to a fresh portion of the standard the requisite amount of iron salt, preferably in the form of ferric-ammonium alum. The amount to be used is calculated as follows: Let $A$ be the number of cubic centimeters of the test solution, $a$ of the standard used, $b$ of the water needed to dilute the standard in the preliminary test, $p$ the weight of iron in terms of $\mathrm{Fe}_{2} \mathrm{O}_{3}$ in $\mathrm{A}$, and $x$ the weight of iron in terms of $\mathrm{Fe}_{6} \mathrm{O}_{3}$ to be added to each cubic centimeter of water used for diluting the standard in the final tests. Then $x=\frac{a+b}{\mathrm{~A} b} p$. For all practical purposes it would probably be sufficient to add the requisite amount of iron salt in solid form to the standard after having diluted it nearly enough to match the test solution, and after it has dissolved to proceed with the test. But this procedure has the disadvantage that each comparison would necessitate the weighing out of a separate portion of iron salt, whereas by the former method the number possible would be limited only by the 
stock of standard and of the prepared ferric-ammonium sulphate solution.

c. alternative mode of PREPaRing the test solution.

As said above (pp. 92, $\gamma$, and 110,12. A. a), the solution that has been used for volumetric determination of total.iron can most conveniently be used for the colorimetric determination of titanium, but if desired this can, of course, be made on some other portion of rock powder. At one time it was the practice in this laboratory to combine it with the determination of barium, as described in Bulletin No. 148, by decomposing the powder by sulphuric and hydrofluoric acids, ${ }^{a}$ expelling the latter by repeated evaporations with sulphuric acid, taking up with dilute sulphuric acid, ${ }^{b}$ filtering from barium sulphate, etc., and estimating the titanium colorimetrically in the filtrate. The expulsion of fluorine must be thorough, or else the titanium result will be low, as already stated (p. 111), and it is not always easy to effect this complete removal, though the time required to do so seems to be in no slight degree dependent on the nature of the fluorides to be decomposed. Long after every trace of fluorine seems to be gone, the formation of a crust on the evaporating solution sometimes allows an accumulation of enough hydrofluoric-acid gas to become plainly manifest to the smell on breaking the crust.

\section{B. GRAVIMETRIC METHODS.}

a. GOOCH'S METHOD.

$\alpha$. Description of the method.-When titanium is present in excess of 4 to 5 per cent and whenever for any reason it is desired to employ a gravimetric method, among the few that have been thoroughly tested that of Doctor Gooch ${ }^{c}$ is unequaled. With one or two minor modifications introduced by Dr. T. M. Chatard, ${ }^{d}$ it is as follows:

Any solution of the rock freed from silica can be used, and the first step is to remove the iron. This is best done, after adding tartaric acid and reducing the iron by means of hydrogen sulphide to the ferrous condition, by rendering the solution ammoniacal and introducing more hydrogen sulphide. If the iron is not thus reduced before precipitation, titanium will be in part thrown down also. ${ }^{e}$ The amount

\footnotetext{
$a$ It is to be borne in mind that evaporation with hydrofluoric acid alone results in loss of titanium by volatilization, but that there is no loss if excess of sulphuric acid is also present.

$b$ With acid rocks solution is very complete, and it can be made nearly so with the most basic by transference to a small beaker and gentle boiling. The residue thus obtained may contain, besides barium sulphate, a little calcium sulphate, zircon, andalusite, topaz, and possibly a trace of titanium in some form. 'It is therefore to be thoroughly fused with sodium carbonate, leached with water, fused with potassium pyrosulphate, dissolved in dilute sulphuric acid, filtered, and the filtrate added to the main one. The insoluble matter will now be chiefly barium sulphate, for the further treatment of which see 13. A (p. 116).

c Proc. Am. Acad. Arts Sci., n. s., vol. 12, p. 435; Bull. U. S. Geol. Survey No. 27, 1886, p. 16; Chem. News, vol. 52, 1885, pp. 55, 68.

d Am. Chem. Jour., vol. 13, 1891, p. 106; Bull. U. S. Geol. Survey No. 78, 1891, p. 87; Chem. News, vol. 63,1891, p. 267.

$e$ Cathrein, A., Zeitschr. f. Kryst., vol. 6, 1882, p. 243; vol. 7, 1883, p. 250.
}

Bull. $305-07-8$ 
of tartaric acid is to be gaged according to the combined weights of the oxides to be held by it in solution, and three times this weight is ample. After removing the iron sulphide by filtration-little washing suffices, because of the relatively small amount of titanium commonly present-the tartaric acid is destroyed as follows:

Potassium permanganate to the extent of two and one-half times the weight of the tartaric acid used is made into a strong solution, and to the ammoniacal filtrate from the iron sulphide enough sulphuric acid is introduced to leave some excess after all the permanganate has been reduced. After expulsion of hydrogen sulphide by boiling, the permanganate is added gradually to the hot solution contained in a large beaker or flask. A vigorous reaction ensues. When a permanent brown precipitate of manganic hydrate appears, the tartaric acid has been fully broken up, and the precipitated manganese is to be redissolved by a few drops of ammonium bisulphite or of sulphurousacid solution.

Ammonia is then added in slight excess, followed at once by acetic acid in considerable excess, and the boiling is continued for a few minutes. Thereby the titanium is freed from most of the alumina, and from lime and magnesia if they had not been earlier removed, also from most of the manganese introduced. The precipitate is filtered and washed with water containing acetic and sulphurous acids, then ignited,fused thoroughly with sodium carbonate, and leached with water to remove phosphoric acid and most of the remaining alumina. The residue is again ignited and fused with sodium carbonate. To the cooled melt in the crucible strong sulphuric acid is to be added, wherein it dissolves readily by aid of gentle heat. This solution is to be poured into a small volume of cold water and the platinum it contains precipitated by hydrogen sulphide at or near boiling temperature. After filtering and cooling, ammonia is added till the titanium is just precipitated, and a measured volume, containing a known weight of absolute sulphuric acid, is then added-just enough to redissolve the precipitate. The solution is then made up with acetic acid in such amount that the final bulk shall contain from 7 to 11 per cent of absolute acid, and then enough solid sodium acetate is stirred in to more than take up the sulphuric acid introduced. On rapidly bringing the liquid to ebullition the titanium is precipitated in flocculent and easily filterable condition, and the precipitation is complete after a minute's boiling, provided all the prescribed conditions have been followed and zirconium is absent.

The precipitate is washed first with acetic acid of 7 per cent strength and then with hot water. After fifteen to twenty minutes' ignition over a good burner it is in condition for weighing and will lose no more weight over the blast lamp. For large amounts of titanium a repetition of the sodium-carbonate fusion, etc., should be made. The 
actual carrying out of all these operations, when once the method is understood, requires much less time than the detailed description would indicate.

' $\beta$. Gooch's method not directly applicable to rocks containing zirconium.-Prior to the adoption of the colorimetric method, Doctor Gooch's was invariably used in this laboratory. Occasional inability to secure clean and complete precipitation by it was experienced, especially with a certain series of rocks rather poor in titanium. Long research showed the difficulty to be due to the presence of zirconium, which acts as a marked preventive of the precipitation of titanium by boiling in an acetic-acid solution under the conditions of the Gooch method.

The above rocks were found to contain up to 0.2 per cent of $\mathrm{ZrO}_{2}$, and this amount was able to prevent precipitation of 0.3 per cent of $\mathrm{TiO}_{2}$. The titanium which came down in excess of this amount did not settle out in flocculent condition, as happens when zirconium is not present, and it was difficult to filter. After the removal of the zirconium, however, in the manner to be hereafter described (14. A, p. 118), no difficulty was experienced in precipitating all the titanium with the usual ease.

b. BASKERVILLE'S METHOD.

C. Baskerville ${ }^{a}$ has proposed the separation of titanium from iron and aluminum by boiling the neutralized solution of the chlorides for a few minutes in presence of sulphurous acid. The test separations as given by him are sharp, and a single precipitation is said to suffice, the titanium being free from iron and easily filterable. This last statement and the ready precipitability are fully confirmed by the experiments of the writer on titaniferous iron ores, but, although the titanium is completely thrown out, it carries with it a little iron, for instance, about 0.25 per cent $\mathrm{Fe}_{2} \mathrm{O}_{3}$ with 8 to 10 per cent $\mathrm{TiO}_{2}$. Zirconium would probably be likewise precipitated (14. B, p. 120) and phosphorus perhaps also, but this last point has not been investigated, neither has the applicability of the method to aluminous rocks been tested.

c. METHOD OF DITTRICH AND POHL.

Attention has been directed (8. C. b, p. 94) to the error resulting from attempting to separate aluminum from titanium by either fused or dissolved sodium hydroxide in absence of appreciable amounts of iron. When iron is present, the procedure given by Dittrich ${ }^{b}$ may be used. It involves fusion in a silver crucible of the ignited oxides of aluminum, iron, etc., obtained in the usual way, with sodium

$a$ Jour. Am. Chem. Soc., vol. 16, 1894, p. 427.

bAnleitung zur Gesteinsanalyse, 1905, pp. 9-31; also Dittrich, M., and Pohl, R., Zeitschr. anorg. Chemíe, vol. $43,1905, \mathrm{p} 236$. 
hydroxide, re-solution of the insoluble matter in hydrochloric acid, precipitation of the iron as sulphide from an ammoniacal tartrate solution, destruction of the tartrate, precipitation of titanium (and zirconium) by ammonia, and determination of the titanium colorimetrically after ascertaining the combined weight of the two oxides.

\section{SUPERIORITY OF THE COLORIMETRIC AND GOOCH METHODS OVER THE OLDER ONES.}

In view of the good results obtainable by the colorimeter method in all cases, and by the Gooch method in the absence of zirconium, it is inexplicable that the old method of precipitation by many hours' boiling in a nearly neutral sulphate solution in presence of sulphurous acid should still find adherents in any part of the world.

13. BARIUM (ZIRCONIUM, RARE EARTHS, TOTAL SULPHUR, CHROMIUM).

These five constituents can with great economy of time and labor be determined in the same portion of rock powder, of which a 2-gram portion should be used, though if vanadium is to be looked for it will be best to determine it and chromium in a different portion (18.C, p. 127). It has been said above (10. C, p. 102) that only in very exceptional cases will barium be found with the calcium and strontium after two or possibly three precipitations of the latter as oxalate, since it passes into the filtrates with the magnesium, whence it may be obtained as sulphate after removal of ammoniacal salts. Addition of some alcohol insures also the recovery of traces of strontium if the rocks are very rich in it; but it is unsafe to regard the amount of barium thus separated from the magnesium as representing the total amount in the rock. It will almost always be found lower than the truth, probably for the reason that there are opportunities during the analysis for slight losses.

\section{A. THE GENERAL METHOD.}

Decomposition is effected by fusing the 2-gram portion with sulphurfree sodium carbonate and an amount of niter insufficient to injure the crucible first over the Bunsen flame, then over the inclined blast, the crucible, being fitted snugly into a hole in a disk of platinum foil or asbestos board (fig. 3, p. 30) to prevent access of sulphur from the gas flame. In case sulphur is not to be regarded, the niter and disk are omitted. After thorough disintegration of the melt in water, to which a drop or two of methyl or ethyl alcohol has been added for the purpose of reducing manganate, the solution is filtered and the residue washed with a very dilute solution of sodium carbonate free from bicarbonate. This is to prevent turbid washings. A yellow color in the filtrate indicates chromium. 
(For the further treatment of the filtrate see "Sulphur," 24.B, p. 160, and "Chromium," 17.B, p. 124.)

The residue is washed from the paper into a small beaker without removing the filter from the funnel and digested with little more than enough warm dilute sulphuric acid to effect solution of all soluble constituents (stronger acid may be used and in larger amount if barium only is sought). A few drops of sulphurous acid are added at the same time to effect solution of the brown hydroxide of manganese. There will remain undissolved more or less residue. Care must be taken not to digest so long with acid as to cause gelatinization of the dissolved silica. The liquid is passed through the original filter and collected in an Erlenmeyer flask of 100 to $150 \mathrm{~cm}^{3}$. The paper with its contents after washing is ignited, evaporated with hydrofluoric and sulphuric acids together, and the final residue taken up with a little hot dilute sulphuric acid. All the barium will remain undissolved, besides some of the strontium and perhaps a good deal of calcium. This is collected on a small filter and the filtrate added to the former one, which now contains all the zirconium and rare earths. (For its further treatment see 14.A, p. 118, and 15.A, p. 120.)

The ignited residue last obtained is fused with sodium carbonate, - leached with water, and the residue dissolved off the filter by a few drops of hydrochloric acid, from which solution the barium is thrown out by a large excess of sulphuric acid. A single solution of the ignited barium sulphate in concentrated sulphuric acid and reprecipitation by water suffices to remove traces of calcium which might contaminate it if the rock was one rich in calcium, and even strontium is seldom retained by it in quantity sufficient to give concern. Should this be the case, however, which will occur when the $\mathrm{SrO}$ and $\mathrm{BaO}$ are together in the rock in, roughly speaking, 0.2 and 0.4 per cent respectively, the only satisfactory way is to convert the sulphates into chlorides and to apply to the mixture the ammonium-chromate method of separation (10.D, p. 103).

Barium and strontium sulphates can be brought into a condition for testing spectroscopically by reducing for a very few moments the whole or part of the precipitate on a platinum wire in the luminous tip of a Bunsen burner, then moistening with hydrochloric acid, and testing in the usual manner.

The procedure outlined in the foregoing paragraphs for the estimation of calcium, strontium, and barium in silicate rocks is the one which long experience has shown to be best adapted for securing the most satisfactory results with a minimum expenditure of time. ${ }^{a}$ Even where no attempt is made to separate contaminating traces of strontium and barium one from the other, the error is usually of no

$a$ For details consult Hillebrand, W. F., Jour. Am. Chem. Soc., vol. 16, 1894, p. 83; Chem. News, vol. 69, 1894, p. 147 . 
great consequence, for an absolute error of even 25 per cent in a substance constituting only 0.1 or 0.2 per cent of a rock is ordinarily of small moment compared with the ability to certify to its presence with approximate correctness.

With such small amounts of barium as are usually found in rocks it is doubtful if Mar's ${ }^{a}$ method for the separation of barium from calcium and magnesium by the solvent action of concentrated hydrochloric acid mixed with 10 per cent of ether on the chlorides could be conveniently applied here, although-for larger amounts the method would seem to be accurate and easily executed. Moreover, it would probably not entirely remove contaminating strontium, and hence offers no advantage.

\section{B. THE METHOD WITHOUT REGARD TO ZIRCONIUM AND SULPHUR.}

If zirconium and sulphur are not to be looked for, the simplest procedure is to decompose the powder by sulphuric and hydrofluoric acids (see A.c, p. 113, under "Titanium"), and to complete the purification of the barium sulphate thus obtained in the manner described in the fourth paragraph of A above.

\section{ZIRCONIUM.}

This element is rarely looked for by chemists, though shown by the microscope to be one of the most constant rock constituents, usually in the form of zircon, in which occurrence its amount can be approximately judged and a chemical test rendered almost unnecessary; but sometimes it occurs in other minerals, and is then unrecognizable under the microscope. It may in most exceptional cases be present up to a few per cent of the rock, rarely reaches 0.2 per cent, and is usually much less than 0.1 per cent.

\section{A. AUTHOR'S METHOD.}

For its detection and estimation in such cases, or whenever a search for it seems called for, the following procedure, based on a method used by G. H. Bailey, ${ }^{b}$ has been devised, which serves, when carried out with care, to detect with certainty the merest trace-0.02 per cent, for instance-in 1 gram.

The preliminary treatment of the rock powder has been fully given under "Barium" (13.A, p. 116), where the separation from barium has been described and also the concentration of the zirconia in a small amount of very dilute sulphuric solution. This should probably not contain much above 1 per cent of sulphuric acid, though the actually permissible limit has not been established. To the solution, which should be in a small flask, is now added hydrogen peroxide to oxidize 
the titanium, and then a few drops of a soluble orthophosphate solution. The flask is set aside in the cold for twenty-four to forty-eight hours. If the color bleaches after a time, more hydrogen peroxide should be added. Under these circumstances the zirconium is thrown out as phosphate and collects as a flocculent precipitate, which at this stage is not always pure. (For the treatment of the filtrate see "Rareearth metals," 15.A, p. 120.) No matter how small or insignificant, it is collected on a filter, ignited, fused with sodium carbonate, leached with water, the filter again ignited, fused with very little acid potassium sulphate, brought into solution in hot water with a few drops of dilute sulphuric acid, poured into a flask or beaker of about. $20 \mathrm{~cm} .^{3}$ capacity, a few drops of hydrogen peroxide and of sodium phosphate are added, and the flask set aside. Titanium is now almost never present, and the zirconium soon appears as a colorless flocculent precipitate, which can be collected and weighed as phosphate. For the small amounts usually met with it is safe to assume that it contains 50 per cent of $\mathrm{ZrO}_{2}$ (51.8 by theory). If the amount is rather large, it may be fused with sodium carbonate, leached, ignited, fused with acid potassium sulphate, reprecipitated by ammonia, and weighed as $\mathrm{ZrO}_{2}$. Certainty as to its identity can be had by again bringing it into solution, precipitating by ammonia, dissolving in hydrochloric acid, evaporating to a drop or two, and testing with turmeric paper or by a microchemical reaction., With the very smallest amounts no color can be obtained by this turmeric-paper test, which, however, responds readily to as little as 1 milligram of dioxide and with proper care for as small an amount as 0.3 milligram (Dr. H. N. Stokes). No element other than thorium is ever likely to contaminate the zirconium thus precipitated.

In Bailey's experiments the precipitation was not made by addition of a phosphate, but is said to be due solely to the hydrogen peroxide, the precipitate being a hydrated peroxide, $\mathrm{Zr}_{2} \mathrm{O}_{5}$, or $\mathrm{ZrO}_{3} .{ }^{a}$ My own efforts as well as those of others to secure a precipitate in acid solutions of zirconium sulphate by hydrogen peroxide alone have been unsuccessful, except in very concentrated solutions and with 30-per cent peroxide. The ability to obtain the zirconium free from phosphoric acid would certainly be a great improvement on the method described above.

Were it not for the necessity of precipitating the zirconium in a weakly acid solution, its separation could be made in the same portion in which the titanium is colorimetrically determined.

\section{B. OTHER METHODS.}

G. Streit and B. Franz ${ }^{b}$ claim to secure complete separation of titanium from iron and zirconium by boiling the neutralized solutions of the sulphates with a large excess (50 per cent) of acetic acid. The 
method has been from time to time recommended, but without any data showing its value. The single separation made by Streit and Franz was far from perfect.

J. T. Davis ${ }^{a}$ separated zirconium sharply from aluminum, but not from iron, by precipitation as an oxyiodate in a boiling neutralized solution of chlorides, but the method is hardly applicable for rock analysis.

C. Baskerville ${ }^{b}$ has proposed a method for the separation of zirconium from iron and aluminum similar to his method for the separation of titanium from those elements (12.B.b, p. 115). - It is based on the precipitability of zirconium by boiling the neutralized chloride solution for two minutes in presence of sulphurous acid, and seems to be excellent. As titanium is always present and is presumably quantitatively thrown down also, the two would have to be separated by hydrogen peroxide. No tests as to the availability of the method for separating the small amounts met with in rock analysis have been made.

The method of M. Dittrich and R. Pohl for determining zirconium by difference, after ascertaining gravimetrically the combined weight of zirconium and titanium and by colorimetry that of titanium, has been outlined on page 115 , under c.

\section{RARE-EARTH METALS OTHER THAN ZIRCONIUM.}

For the cases in which it may be necessary to look for rare earths other than zirconia, the following procedures will prove satisfactory and not at all difficult.

\section{A. USUAL METHOD.}

The filtrate from the zirconium phosphate (14.A, p. 118) or, if zirconium is not to be determined, the earlier solution (13.A, p. 116), is treated with excess of potassium hydroxide to precipitate the rare earths, and ferric and titanic oxides, while retaining the silica and alumina in solution. After settling the liquid is decanted and the precipitate is washed once or twice by decantation and slightly on the filter. The precipitate is washed from the paper into a small platinum dish, treated with hydrofluoric acid, and the liquid evaporated nearly to dryness. A little water with a few drops of hydrofluoric acid is added and the insoluble rare-earth fluorides (crude) collected on a small filter held by a perforated platinum or rubber cone and washed with water acidified with the same acid. The precipitate is washed into a small platinum dish and evaporated to dryness with sulphuric acid, the paper being burned and added before expulsion of the acid. The sulphates are dissolved in dilute hydrochloric acid, the earth hydroxides precipitated by ammonia, redissolved in hydro- 
chloric acid, the solution evaporated to dryness, and the residue heated with a few drops of a solution of oxalic acid. Anything that may be present except the rare earths dissolves readily, these remaining as insoluble oxalates. If there is a doubt as to the complete separation of the alkaline earths the ignited oxalates may be dissolved in hydrochloric or nitric acid, precipitated by ammonia, redissolved in whichever one of the acids may have been used, the solution evaporated to dryness, the oxalates reprecipitated, ignited, and the resulting oxides weighed.

The amounts are too small to permit separation into further groups, but the presence of cerium can be readily shown if a strong yellow color develops on heating the ignited oxides with strong sulphuric acid.

\section{B. ALTERNATIVE METHOD.}

The rock powder is thoroughly decomposed by several partial evaporations with hydrofluoric acid, the transparent precipitate of silico-fluorides and of the fluorides of all earth metals except zirconium is collected on a platinum cone, washed with water acidulated by hydrofluoric acid, washed back into the dish or crucible, and evaporated with enough sulphuric acid to expel all fluorine. The filter is burned and added. By careful heating the excess of sulphuric acid is removed and the sulphates are taken up by dilute hydrochloric acid. The rare earths, with perhaps some alumina, are then precipitated by ammonia, washed, redissolved in hydrochloric acid, and evaporated to dryness, then taken up with water and a drop of hydrochloric acid, and only enough ammonium acetate to neutralize the latter added, followed by oxalic acid (not ammonium oxalate, which would fail to precipitate thorium). In this way as little as 0.03 per cent of rare earths has been found when working on not more than 2 grams of material.

\section{PHOSPHORUS.}

It is sometimes possible to extract all phosphorus from a rock by simple digestion with nitric acid, but quite as often, if not oftener, this fails; hence the necessity for resorting to one of the longer methods of extraction detailed below. Whatever method is used great care is required in order to secure accurate results.
a. PROCEDURE WHEN MATERIAL is ample.
a. WASHINGTON'S METHOD OF PRELIMINARY TREATMENT.

About 1 gram of rock powder is decomposed in a platinum dish or capacious crucible with dilute nitric and hydrofluoric acids. When all gritty particles have disappeared the solution is evaporated to dryness and reevaporated with nitric acid two or three times to 
decompose fluorides and silicofluorides to a considerable extent. ${ }^{a}$ The residue is treated on the bath with dilute nitric acid and eventually filtered from any undissolved matter. This last should be for precaution's sake always ignited, fused with a little sodium carbonate, extracted with water, the extract acidified with nitric acid and added to the main solution. (For further treatment see c, below.)

\section{b. ALTERNATIVE METHOD OF PRELIMINARY TREATMENT.}

Fuse with sodium carbonate, separate silica by a single evaporation with nitric acid, treat the ignited silica with hydrofluoric and nitric acids, evaporate to expel hydrofluoric acid, repeat the evaporation with nitric acid alone two or three times, bring the small residue into solution by boiling with nitric acid and add it to the main portion.

c. SUBSEQUENT TREATMENT.

Add to the solution obtained in a or $\mathrm{b}$ ammonium nitrate and molybdate solution as ordinarily prescribed.

The turbidity often observed on dissolving the precipitated and washed phosphomolybdate in ammonia is due to a compound of phosphorus. If the addition of a small fragment of a crystal of eitric or tartaric acid fails to dissolve it, this should always be re-fused with sodium carbonate, extracted with water, and the filtrate otherwise treated as above, in order to secure the phosphorus in it.

According to F. A. Gooch and M. Austin, ${ }^{b}$ in order to secure a magnesium-ammonium phosphate of normal composition, the procedure at this point should be as follows: To the phosphate solution, containing not more than 5 to 10 per cent of ammonium chloride and a slight excess of magnesia mixture, a little ammonia is added, and the precipitate is washed in due time with weak ammonia water. In general, however, as these conditions can seldom be fulfilled, they recommend to decant the supernatant liquid through the filter which is later to receive the precipitate, to dissolve this in as little hydrochloric acid as possible, to reprecipitate by dilute ammonia without further addition of magnesia mixture, and to wash finally with weakly ammoniacal water. Excess of ammonia, of ammonium salts, and of precipitant are all objectionable. In rock analysis the second precipitation will seldom be necessary. (For ignition, etc., of the precipitate, see this subject under "Magnesium," 11. B. a, p. 108.)

\section{B. PROCEDURE WHEN MATERIAL IS SCANTY.}

The following procedure admits of determining in the same portion, besides phosphorus, barium, iron, vanadium, chromium, and titanium,

\footnotetext{
$a$ The method as originally described in Washington's Manual of the Chemical Analysis of Rocks has been modified by Doctor Washington and myself independently to the extent of doing away with the filtration of the precipitate of silicofluorides and fluorides before proceeding with the evaporation.

b $\Lambda$ m. Jour. Sci., 4th ser., vol. 7, 1899, p. 187; Z'eitschr. anorg. Chemie, vol. 20, 1899, p.121; Chem. News, vol. 79,1899 , pp. $233,244,255$.
} 
the last two either colorimetrically or gravimetrically, and is in large part extracted from a paper by Dr. T. M. Chatard. ${ }^{a}$

Silica is removed by hydrofluoric and sulphuric acids, excess of fluorine expelled, the residue brought into solution as far as possible with sulphuric or hydrochloric acid and hot water, filtered, the residue ignited, fused with sodium carbonate, dissolved in hydrochloric acid, and the solution, after precipitation of barium, added to the main one, which is now precipitated by ammonia to get rid of the magnesium salts usually present and thus insure a cleaner subsequent fusion with sodium carbonate.

The precipitated $\mathrm{Al}_{2} \mathrm{O}_{3}, \mathrm{P}_{2} \mathrm{O}_{5}, \mathrm{Cr}_{2} \mathrm{O}_{3}, \mathrm{Fe}_{2} \mathrm{O}_{3}$, and $\mathrm{TiO}_{2}$ are dissolved in hot hydrochloric acid and filtered into a large platinum crucible, the filter burned and added, the solution evaporated to pastiness, a little water added to dissolve the salts, and dry sodium carbonate added in portions and stirred in thoroughly to prevent lumpiness in the fusion to follow, which is continued for half an hour. Addition of sodium nitrate is not necessary.

The fused mass is boiled out with water and washed with very dilute sodium-carbonate solution. In the residue iron and titanium can be determined by the methods already described. In the filtrate chromium can be determined colorimetrically if present in sufficient amount to give a pronounced color (see 17. B, p. 124). Afterwards, or immediately if the chromium is not to be thus estimated, enough ammonium nitrate is added to react with all the carbonate and the solution is digested on the bath till most of the ammonium carbonate is gone. Nearly if not quite all alumina is thus thrown out, carrying with it all phosphorus. The precipitate is washed with dilute ammonium-nitrate solution till the yellow color wholly disappears, after which it is dissolved in nitric acid and the phosphorus thrown out by molybdate solution. The filtrate from the aluminum and phosphorus, containing chromium and vanadium, can be treated as detailed in the following sections.

\section{CHROMIUM.}

If vanadium is absent, or nearly so, as is apt to be the case in those highly magnesian rocks (peridotites), which usually carry a good deal of chromium, the following separation and gravimetric method for chromium gives good and concordant results, but in the presence of vanadium, and it is best generally to assume its presence, the colorimetric method should always be adopted.

a Am. Chem. Jour., vol. 13, 1891, p. 106; Bull. U. S. Geol. Survey No. 78, 1891, p. 87; Chem. News, vol. 63,1891 , p. 267. 


\section{A. GRAVIMETRIC METHOD.}

Having obtained chromium in solution as chromate and free from all else but a little alumina, as at the conclusion of the preceding section on phosphorus, proceed as follows:

Concentrate if necessary and add fresh ammonium sulphide, or introduce hydrogen sulphide. The chromium is reduced and appears as a precipitate of sesquioxide mixed with the rest of the alumina. This precipitate is now treated according to $\mathrm{H}$. Baubigny ${ }^{a}$ by dissolving in nitric acid, evaporating nearly to dryness, and heating with strong nitric acid and potassium chlorate, finally evaporating to dryness to get rid of the acid. Oxidation is complete and very speedy. On dilution with cold water, acid sodium carbonate is added in slight excess, and after two or three hours the precipitated alumina is filtered off. From the filtrate the chromium is then thrown out by fresh ammonium sulphide, redissolved, reprecipitated to free from alkali, and weighed.

The separation of aluminum from chromium by hydrogen peroxide in ammoniacal solution, as recommended by P. Jannasch and E. v. Cloedt, ${ }^{b}$ has been shown by C. Friedheim and E. Brühl ${ }^{c}$ to be valueless.

\section{B. COLORIMETRIC METHOD.}

For this very accurate and by far the quickest method ${ }^{d}$ for determining chromium in rocks and ores where the amount does not exceed a few per cent, there is needed the aqueous extract of a sodium-carbonate fusion of the rock (as obtained, for instance, under "Phosphorus," B, p. 122) in order to compare its color with that of a standard solution.

$\alpha$. Preparation and strength of standard solution.- This standard solution is made by dissolving 0.25525 gram or double that amount of pure potassium monochromate in 1 liter of water made alkaline by a little sodium carbonate. Each cubic centimeter then corresponds to 0.1 or 0.2 milligram of chromic oxide $\left(\mathrm{Cr}_{2} \mathrm{O}_{3}\right)$, in which condition chromium is usually reported in rocks and ores. It is probably inadmissible to increase the strength of the standard much above the figure given.

$\beta$. Preparation of the test solution.-Before filtering the aqueous extract of the sodium-carbonate fusion a few drops of alcohol (ethyl or methyl) are added to destroy the color of sodium manganate. If the yellow color of the filtrate is very faint, concentration by evaporation will strengthen it, and less than 2 milligrams of chromic

a Bull. Soc. chimique, n. s., vol. 42, 1884, p. 291; Chem. News, vol. 50, 1885, p. 18.

$b$ Zeitschr. anorg. Chemie, vol. 10, 1895, p. 402.

$c$ Zeitschr. anal. Chemie, vol. 38,1899 , p. 681.

$d$ Hillebrand, W. F., Jour. Am. Chem. Soc., vol. 20, 1898, p. 454; Chem. News, vol. 78, 1898, pp. 227, 239; Bull. U. S. Geol. Survey No. 167, 1900, p. 37. First applied by L. de Koningh (Nederl Tyds. voor Pharm., Chem. en Tox., 1889) for the estimation of chromium in foodstuffis. 
oxide in 1 gram of rock can then be exactly measured. For smaller amounts it is best to employ from 3 to 5 grams of powder and then to concentrate the chromium by precipitation by mercurous nitrate, as detailed in the next section under "Vanadium" (18. C, p.127); otherwise it may be difficult or impossible, because of the large amount of alkali carbonate present, to obtain a filtrate of sufficiently small bulk to show a decided color.

If niter has been used in the fusion and the crucible has been at all attacked by it, a yellow coloration of the filtrate may be due to dissolved platinum, but neither the proportion of niter nor the temperature of the blast should ever be high enough to permit the crucible to be attacked. A passing yellowish coloration of the filtrate, due to this or some other cause, is not to be ascribed to chromium.

$\gamma$. Comparison of colors. - The final solution is transferred to a graduated flask of such size that its color shall be weaker than that of the standard chromium solution. Definite amounts of the latter are then diluted with water from a burette until of the same strength as the test solution, exactly as described on page 32 . For very minute amounts it may be necessary to use Nessler tubes, as in ammonia estimations, instead of the glasses and apparatus there described and depicted.

As with colorimetric methods in general, this one gives better results with small than with large percentages of chromium, yet it can be applied in the latter cases with satisfactory results by making a larger number of consecutive comparisons with the same solution. ${ }^{a}$

\section{A FEW COMPARATIVE DATA.}

A few comparisons between colorimetric and gravimetric determinations of chromium are here given to show the order of agreement, the former having been made several months and even years after the latter.

Percentage determinations of chromium $\left(\mathrm{Cr}_{2} \mathrm{O}_{3}\right)$.

\begin{tabular}{|r|c|}
\hline Gravimetric. & Colorimetric. \\
\hline Trace. & 0.018 \\
0.05 & .051 \\
.14 & .12 \\
.08 & .083 \\
Trace. & .013 \\
None. & .0086 \\
None. & .0067 \\
\hline
\end{tabular}

The outcome was somewhat surprising, for it was hardly to be expected that the long and laborious quantitative separations should have resulted so well. It should be mentioned that for the gravi-

$a$ D. W. Horn has studied the "Variable sensitiveness in the colorimetry of chromium." See Am. Chem. Jour., vol. 35, 1906, p. 253. 
metric tests but 1 or 2 grams at most were used, which accounts for the reported absence of chromium in two instances, this report being based on the lack of color in the aqueous extract of the alkali fusion after removal of manganese.

\section{VANADIUM (CHROMIUM) AND MOLYBDENUM.}

\section{A. DISTRIBUTION OF VANADIUM AND MOLYBDENUM.}

The wide distribution of vanadium throughout the earth's crust has in recent years been clearly established (see pp. 18-19), not only in ores and in coals, but in clays, limestones, sandstones, and igneous rocks. ${ }^{a}$ I have shown $^{b}$ that vanadium occurs in appreciable amounts in the more basic igneous and metamorphic rocks up to 0.08 per cent or more of $\mathrm{V}_{\cdot 2} \mathrm{O}_{3}$, but that it seems to be absent or nearly so from the highly siliceous ones. Some of their ferric aluminous silicate constituents carry still higher percentages-up to 0.13 per cent $\mathrm{V}_{2} \mathrm{O}_{3}$ in a biotite separated from a pyroxenic gneiss. Molybdenum, on the other hand, appears to be confined in quantities susceptible of detection to the more siliceous rocks, and, except perhaps in rare instances, is not present in them in quantitatively determinable amount when operating on 5 grams of material. Hence the quantitative search for vanadium will usually be limited to rocks with less than 60 per cent of silica. The search for it even then will perhaps not often warrant the necessary expenditure of time, but in this connection it is to be remembered that neglect to determine it introduces an error in the figures for both ferrous and ferric oxides, which in extreme cases may be of considerable moment (see 7. B. b, p. $92 ; 19$. C. c. $\beta$, p. 140).

\section{B. CONDITION OF VANADIUM IN ROCKS.}

The connection below and elsewhere mentioned of vanadium with the ferric aluminous silicates of rocks, taken in connection with the existence of the mineral roscoelite, classed as a vanadium mica, indicates a condition of the vanadium corresponding to aluminum and ferric iron, and that it is to be regarded as replacing one or both of these elements. Hence it should be reported as $\mathrm{V}_{2} \mathrm{O}_{3}$ and not as $\mathrm{V}_{2} \mathrm{O}_{5}$.

What its condition may be in matter of secondary origin, like clays, limestones, sandstones, coals, and ores of iron, is yet open to discussion. It was my opinion at one time that it should be regarded as in the pentavalent state $\left(\mathrm{V}_{2} \mathrm{O}_{5}\right)$, but my work on certain remarkable vanadiferous sandstones ${ }^{c}$ of western Colorado, in which it unquestionably

a Hillebrand, W. F., Distribution and quantitative occurrence of vanadium and molybdenum in rocks of the United States: Am. Jour. Sci., 4th ser., vol. 6, 1898, p. 209; Chem. News, vol. 78, 1898, p. 216; Bull. U. S. Geol. Survey No. 167, 1900, p. 49.

$b$ Loc. cit.

c Hillebrand, W. F., and Ransome, F. L., Am. Jour. Sci., 4th ser., vol. 10, 1900, p. 120. 
occurs as trivalent vanadium $\left(\mathrm{V}_{2} \mathrm{O}_{3}\right)$, has led to a decided unsettling of this view. It is but proper to recall that $\mathrm{C}$. Czudnowicz, ${ }^{a}$ because of the extreme difficulty in completely extracting it from iron ores by an alkali-carbonate fusion and because of the easy reducibility of vanadic acid by ferrous salts, under the conditions in which brown iron ores are supposed to form, considered the vanadium in such ores to be in a lower condition of oxidation $\left(\mathrm{V}_{2} \mathrm{O}_{3}\right)$. O. Lindemann's ${ }^{b}$ contrary conclusion with regard to certain iron ores, because the vanadium was extracted as $\mathrm{V}_{2} \mathrm{O}_{5}$ by sodium-carbonate fusion without niter, is not valid, since this would probably be the case even if it existed in the ore as $\mathrm{V}_{2} \mathrm{O}_{3}$.

\section{AUTHOR'S METHOD.}

a. DESCRIPTION OF THE METHOD.

In the following method there is nothing absolutely novel except that chromium and vanadium, when together, need not be separated, but are determined, the former colorimetrically, as already described (17. B., p. 124), the latter volumetrically, in the same solution. ${ }^{c}$

Five grams of the rock are thoroughly fused over the blast with 20 of sodium carbonate and 3 of sodium nitrate. After extracting with water and reducing manganese with alcohol it is probably quite unnecessary, if the fusion has been thorough, to remelt the residue as above, though for some magnetites and other ores containing larger amounts of vanadium than the generality of rocks, this may be necessary, as Edo Claassen has shown. ${ }^{d}$ The aqueous extract is next nearly neutralized by nitric acid, the amount to be used having been conveniently ascertained by a blank test with exactly 20 grams of sodium carbonate, etc., and the solution is evaporated to approximate dryness. Care should be taken to avoid overrunning neutrality, because of the reducing action of the nitrous acid set free from the nitrite produced during fusion, but when chromium is present it has been my experience that some of this will invariably be retained by the precipitated silica and alumina, though only in one case have I observed a retention of vanadium, it being then large. The use of ammonium nitrate, instead of nitric acid, for converting the sodium carbonate into nitrate does not seem to lessen the amount of chromium retained by the silica and alumina.

As a precautionary measure, therefore, and always when chromium is to be estimated also, the silica and alumina precipitate should be evaporated with hydrofluoric and sulphuric acids, the residue fused with a little sodium carbonate and the aqueous extract again nearly. neutralized with nitric acid and boiled for a few moments, the filtrate being added to the main one.

$a$ Pogg. Ann., vol. 120, 1863, p. 20.

$b$ Dissertation, Jena, 1878, thru Zeitsch. anal. Chemie, vol. 18, 1879, p. 99.

c Hillebrand, W. F., Jour. Am. Chem. Soc., vol. 20, 1898, p. 461; Chem. News; vol. 78, 1898; p. 295 ; Bull.

U. S. Geol. Survey No. 167, 1900, p. 44.

dAm. Chem. Jour., vol. 8, 1886, p. 437 . 
Mercurous nitrate is now added to the cold alkaline solution in some quantity, so as to obtain a precipitate of considerable bulk, containing, besides mercurous carbonate, chromium, vanadium, molybdenum, tungsten, phosphorus, and arsenic, should all happen to be in the rock. The mercurous carbonate serves to counteract any acidity resulting from the decomposition of the mercurous nitrate. Precipitating in a slightly alkaline instead of a neutral solution renders the addition of precipitated mercuric oxide unnecessary for correcting this acidity. If the alkalinity, as shown by the formation of an unduly large precipitate, should have been too great, it may be reduced by careful addition of nitric acid until an added drop of mercurous nitrate no longer produces a cloud.

After heating and filtering, the precipitate is ignited in a platinum crucible after drying and removing from the paper to obviate any chance of loss of molybdenum and of injury to the crucible by reduction of arsenic. The residue is fused with a very little sodium carbonate, leached with water, and the solution, if colored yellow, filtered into a graduated flask of $25 \mathrm{~cm} .{ }^{3}$ or more capacity. The chromium is then determined accurately in a few minutes by comparing with a standard alkaline solution of potassium monochromate (17. B, p. 124). Then, or earlier in absence of chromium, sulphuric acid is added in slight excess, and molybdenum and arsenic, together with occasional traces of platinum, are precipitated by hydrogen sulphide, preferably in a small pressure bottle. ${ }^{a}$ If the color of the precipitate indicates absence of arsenic the filter with its contents is carefully ignited in porcelain, and the delicate sulphuric-acid test for molybdenum is applied as follows: The molybdenum compound is heated in porcelain with a single drop of strong sulphuric acid till the acid is nearly volatilized. On cooling a beautiful blue color is proof of the presence of molybdenum.

The filtrate, in bulk from $25 \mathrm{~cm}^{3}$ to $100 \mathrm{~cm} .{ }^{3}$, is boiled to expel hydrogen sulphide, and titrated at a temperature of $70^{\circ}$ to $80^{\circ}$ with a very dilute solution of permanganate, representing about 1 milligram of $\mathrm{V}_{2} \mathrm{O}_{5}$ per cubic centimeter, as calculated from the iron strength of the permanganate, one molecule of $\mathrm{V}_{2} \mathrm{O}_{5}$ being indicated for each one of $\mathrm{Fe}_{2} \mathrm{O}_{3}$. One or two checks are always to be made by reducing again, by means of a current of sulphur-dioxide gas, boiling this out again, ${ }^{b}$ and repeating the titration. The latter results are apt to be a very little lower than the first and are to be taken as the correct ones.

\footnotetext{
$a$ From a sulphuric solution the separation of platinum and molybdenum by hydrogen sulphide is much more rapid and satisfactory than from a hydrochloric solution.

$b$ The direct use of a solution of sulphur dioxide or of an alkali sulphite is inadmissible unless these have been freshly prepared, since after a lapse of time they contain other oxidizable bodies than sulphurous acid or a sulphite. The sulphur dioxide is best obtained as wanted by heating a flask containing a solution of sulphur dioxide, or of a sulphite to which sulphuric acid has been added.

The expulsion of the last traces of sulphur dioxide is said to be more effectively accomplished by boiling with simultaneous passage of a rapid current of carbon dioxide for a few minutes at the last than by boiling alone. Because of the small amount of air carried with it, long passage of the gas is said to result in slight oxidation of the vanadium (Manasse, O., Ann. Chem. u. Pharm., vol. 240, 1887, p. 23; Zeitschr. anal. Chemie vol. 32, 1893, p. 225.)
} 
b. CONFIRMATORY QUALITATIVE TESTS.

In case the volume of permanganate used is so small as to make doubtful the presence of vanadium, it is necessary to apply a qualitative test, which is best made as follows: The solution is evaporated and heated to expel excess of sulphuric acid, the residue is taken up with 2 or $3 \mathrm{~cm}^{3}$ of water and a few drops of dilute nitric acid, and a couple of drops of hydrogen peroxide are added. A characteristic brownish tint indicates vanadium. Unless the greater part of the free sulphuric acid has been removed the appearance of this color is sometimes not immediate and pronounced, hence the above precaution. It is also necessary that the nitric acid shall be in considerable excess, since in a neutral or only faintly acid solution the color does not appear strongly.

The above is a surer test to apply than the following: Reduce the bulk to about $10 \mathrm{~cm} .^{3}$, add ammonia in excess, and introduce hydrogen sulphide to saturation. The beautiful cherry-red color of vanadium in ammonium-sulphide solution is much more intense than that caused by hydrogen peroxide in acid solution, but the action of ammonia is to precipitate part or all of the vanadium with the chromium or aluminum that may be present or with the manganese used in titrating, and ammonium sulphide is unable to extract the vanadium wholly from these combinations. Usually, however, the solution will show some coloration, and addition of an acid precipitates brown vanadium sulphide, which can be collected, ignited, and further tested if desired.

c. APPLICATION OF THE METHOD IN PRESENCE OF RELATIVELY MUCH CHROMIUM.

The application of the method in its foregoing simplest form is subject to one limitation-the chromium must not be present above a certain moderate amount. This limitation is due to the considerable amount of permanganate then required to produce a clear transition tint when titrating in a hot solution, as is advisable with vanadium. In a cold solution of chromic sulphate much less permanganate is needed to produce the peculiar blackish tint without a shade of green, which affords a sure indication of excess of permanganate, but in a hot and especially a boiling solution the oxidation of the chromium itself takes place so rapidly that a very large excess of the reagent may be added before a pronounced end reaction is obtained. Nevertheless, fairly satisfactory determinations of as little as 1 or $2 \cdot$ milligrams of vanadium pentoxide can be made in presence of as much as 30 milligrams of chromic oxide. To accomplish this it is only necessary to apply a simple correction obtained by adding permanganate to a like bulk of equally hot chromic sulphate solution containing approximately the same amount of chromium.

Bull. 305-07-9 
C. H. Ridsdale ${ }^{a}$ titrated the cold solution to avoid oxidation of chromium and obtained accurate results, but in my experience the end reaction is then uncertain.

The following tables contain the results of a considerable number of tests, those in Table 2 being tabulated separately in order to show the degree of accuracy attainable with a large excess of chromium by applying the correction mentioned above and also the amount of this correction:

TABLE 1.-Tests for vanadium in the presence of chromium.

\begin{tabular}{|c|c|c|c|c|c|c|c|c|c|}
\hline No. & $\begin{array}{l}\text { Chromic } \\
\text { oxide. }\end{array}$ & $\begin{array}{l}\text { Vanadium } \\
\text { pentoxide. }\end{array}$ & $\begin{array}{l}\text { Vanadium } \\
\text { pentoxide } \\
\text { found. }\end{array}$ & Error. & No. & $\begin{array}{l}\text { Chromic } \\
\text { oxide. }\end{array}$ & $\begin{array}{l}\text { Vanadium } \\
\text { pentoxide. }\end{array}$ & $\begin{array}{l}\text { Vanadium } \\
\text { pentoxide } \\
\text { found. }\end{array}$ & Error. \\
\hline & Milligrams & Milligrams & Milligrams & $M g$. & & Milligrams & Milligrams & Milligrams & $M g$. \\
\hline $\begin{array}{l}1 \\
2\end{array}$ & $\begin{array}{l}1 \\
1\end{array}$ & $\begin{array}{r}9.87 \\
.94\end{array}$ & $\begin{array}{l}9.22 \\
1.04\end{array}$ & $\begin{array}{l}-0.15 \\
+\quad .10\end{array}$ & $\begin{array}{l}7 \\
8\end{array}$ & $\begin{array}{l}3.5 \\
6\end{array}$ & $\begin{array}{c}18.74 \\
5.6\end{array}$ & $\begin{array}{c}18.97 \\
6.1\end{array}$ & \\
\hline 3 & 1.5 & 5.25 & $\begin{array}{r}.98 \\
5.49 \\
5.43\end{array}$ & $\begin{array}{l}+.04 \\
+.24 \\
+\quad 19\end{array}$ & $\begin{array}{r}9 \\
10 \\
11\end{array}$ & $\begin{array}{r}6 \\
6 \\
6 \\
10\end{array}$ & $\begin{array}{l}4.68 \\
5.62 \\
5.62\end{array}$ & $\begin{array}{l}4.78 \\
5.58 \\
558\end{array}$ & $\begin{array}{l} \pm .10 \\
-.04 \\
-.04\end{array}$ \\
\hline 4 & 2 & 5.62 & $\begin{array}{l}5.43 \\
5.5 \\
5.5\end{array}$ & $\begin{array}{l} \pm .12 \\
=.12\end{array}$ & 12 & $\begin{array}{l}10 \\
10\end{array}$ & 23.52 & $\begin{array}{r}\text { 5. } \\
23.81 \\
\mathbf{3}\end{array}$ & $\begin{array}{l}+.04 \\
+.24 \\
+\quad .19\end{array}$ \\
\hline 5 & 3 & 4.68 & $\begin{array}{l}4.78 \\
4.78\end{array}$ & $\begin{array}{r}+10 \\
+.10\end{array}$ & 13 & 10 & 46.85 & $\begin{array}{l}46.98 \\
47.20\end{array}$ & $\begin{array}{l}+.13 \\
+\quad .33 \\
+\quad .3 i\end{array}$ \\
\hline 6 & 3 & 5.62 & $\begin{array}{l}4.83 \\
5.58\end{array}$ & $\begin{array}{l} \pm .15 \\
-.04\end{array}$ & 14 & 25 & 23.52 & $\begin{array}{l}23.65 \\
23.75\end{array}$ & $\begin{array}{l}+.13 \\
+.22\end{array}$ \\
\hline 7 & 3.5 & 18.74 & $\begin{array}{r}5.58 \\
18.89\end{array}$ & $\begin{array}{l}-.04 \\
+\quad .15\end{array}$ & 15 & 87.5 & 23.52 & 23.71 & .19 \\
\hline
\end{tabular}

TABLE 2.-Application of correction for larger amounts of chromium, obtained by addin. potassium permanganate to an equal bulk of solution containing a like amount of chromic sulphate.

\begin{tabular}{|c|c|c|c|c|c|c|}
\hline \multirow{2}{*}{ No. } & \multirow{2}{*}{$\begin{array}{l}\text { Chromic } \\
\text { oxide. }\end{array}$} & \multirow{2}{*}{$\begin{array}{l}\text { Vanadium } \\
\text { pentoxide. }\end{array}$} & \multicolumn{2}{|c|}{$\begin{array}{l}\text { Vanadium pentoxide } \\
\text { found. }\end{array}$} & \multirow{2}{*}{ Error. } & \multirow{2}{*}{$\begin{array}{l}\text { Volume of solu- } \\
\text { tion. }\end{array}$} \\
\hline & & & $\begin{array}{l}\text { Uncor- } \\
\text { rected. }\end{array}$ & Corrected. & & \\
\hline & Milligrams. & Milligrams. & & & Milligram. & \\
\hline 16 & 20 & 0.94 & 1.59 & 0.99 & +0.05 & 50 to $100 \mathrm{~cm}^{3}$ \\
\hline 17 & 20 & 1.87 & $\begin{array}{l}2.69 \\
2.39\end{array}$ & $\begin{array}{l}2.09 \\
1.79\end{array}$ & & 50 to $100 \mathrm{~cm} .^{3}$. \\
\hline & & & 2.59 & 1.99 & +.12 & \\
\hline 18 & 20 & 18.74 & 19.4 & 18. 73 & -.01 & 50 to $100 \mathrm{~cm}^{3}$ \\
\hline & & & $\begin{array}{l}19.0 \\
19.3\end{array}$ & $\begin{array}{l}18.00 \\
18.63\end{array}$ & -.11 & \\
\hline 19 & 30 & 1.87 & 2.99 & 2.14 & +.27 & A bout $100 \mathrm{~cm} .^{3}$ \\
\hline & & & $\begin{array}{l}2.79 \\
2.79\end{array}$ & $\begin{array}{l}1.94 \\
1.94\end{array}$ & $\begin{array}{r}+.07 \\
+\quad .07 \\
+\quad .07\end{array}$ & ADVut \\
\hline & & & 2.69 & 1.84 & -.03 & \\
\hline 20 & 30 & 107 & 2.69 & 1.84 & -.03 & \\
\hline 20 & 30 & 1.87 & $\begin{array}{l}2.69 \\
2.89\end{array}$ & $\begin{array}{l}1.79 \\
2.09\end{array}$ & $\begin{array}{r}-.08 \\
+\quad .22\end{array}$ & $200 \mathrm{~cm}^{3}$ \\
\hline & & & $\begin{array}{l}2.89 \\
2.79\end{array}$ & 2.09 & $\begin{array}{r}+.22 \\
+\quad 12\end{array}$ & \\
\hline 21 & 62 & 46.85 & 48.60 & $\begin{array}{r}1.99 \\
47.60\end{array}$ & $\begin{array}{l}+.12 \\
+.75\end{array}$ & $200 \mathrm{~cm}^{3}$ \\
\hline
\end{tabular}

In spite of the fact that the correction in most of the trials of this last table represents a large proportion of the permanganate used, the results must be considered satisfactory in view of the small amount of vanadium present, and they show that the method in competent hands after a little experience affords trustworthy figures. 
The method of $T$. Fischer $a$-digestion of the precipitated lead salts with a strong solution of potassium carbonate - appears to offer the long-needed satisfactory quantitative separation of arsenic, phosphorus, chromium, tungsten, and molybdenum from vanadium, the normal lead metavanadate remaining quite unattacked, according to Fischer, while the other lead salts are wholly decomposed, but the applicability of this method to the separation of the minute amounts often found in rocks and ores has not been tested. The object has been in the present case to reach satisfactory results with the greatest expedition, and when chromium is not present in considerable amount this is accomplished.

Fortunately, chromium is almost never a prominent constituent of clays, coals, iron ores, and those rocks in which vanadium has thus far been reported, for although it is usually certain of the most basic of the silicate rocks that are highest in chromium - as the peridotitesyet in these, so far as present experience teaches, vanadium is lacking, a fact doubtless connected with the simultaneous absence from them of ferric aluminous silicates.

\section{COLORIMETRIC METHOD FOR VANADIUM.}

In the paper already cited ${ }^{b}$ the possibility of a colorimetric method for vanadium, based on its behavior toward hydrogen peroxide, was suggested. L. Maillard ${ }^{c}$ has since developed such a method, though it has not been tested in the Survey laboratory.

\section{FERROUS IRON.}

\section{A. COMPARISON OF SEALED-TUBE AND HYDROFLUORIC-ACID METHODS- COMPARATIVE WORTHLESSNESS OF THE FORMER IN ROCK ANALYSIS.}

No point in rock analysis has been the cause of greater solicitude to the chemist, and especially to the mineralogist and petrographer, than the determination of iron in ferrous condition. The sealed-tube or Mitscherlich method with sulphuric acid, for a long time the only available one, is in theory perfect, since complete exclusion of oxygen is easily attainable. Its earliest recognized defect lies in the inability to secure always complete decomposition of the iron-bearing minerals, and even to ascertain, oftentimes, whether or not the decomposition has been complete. The addition of hydrofluoric acid to the sulphuric in the tube, in order to insure this breaking up, is to be regarded as of very doubtful utility in most cases, since the glass may be so strongly attacked as to add an appreciable amount of iron to the

a Inaugural Dissertation, Rostock, 1894 .

${ }^{b}$ Hillebrand, W. F., Jour. Am. Chem. Soc., vol. 20, 1898, p. 461; Chem. News, vol. 78, 1898, p. 295, Bull.

U. S. Geol. Survey No. 167,1900 , p. 44.

c Bull. Soc. chim., vol. 23, 1900, p. 559 . 
solution, and the hydrofluoric acid may have exhausted itself in attacking the glass before the more refractory minerals succumb. Nevertheless, if decomposition can be effected by sulphuric acid alone the results obtained are sharp and concordant; but they are in rock analysis usually higher than when made by any of the modifications of the hydrofluoric-acid method now so extensively practised. This difference is not very marked with rocks containing but 1 or 2 per cent of ferrous iron, but it increases with rising percentage to such an extent that where the sealed-tube method will show 12 per cent ferrous oxide the other may indicate no more than 10 per cent. This is a fact of which I have long been cognizant, but it seems not to have been known to chemists or petrographers at large, though E. A. Wülfing ${ }^{a}$ noticed this difference in certain analyses without appreciating its significance. Experiments with soluble iron salts of known composition, like ferrous sulphate and ferrous-ammonium sulphate, throw no light on the subject, for both methods give with them the same sharp and accurate results.

The key to the problem was discovered by L. L. de Koninck ${ }^{b}$ a good many years ago, but remained unknown to the chemical world by reason of its obscure medium of publication until rediscovered by Dr. H. N. Stokes in this laboratory, during an investigation on the action of ferric salts on pyrite and other sulphides. ${ }^{c}$ That oxidation of the sulphide and reduction of the ferric salt hereby takes place was recognized by J. H.L. Vogt, ${ }^{d}$ but not the ease with which the change takes place and the completeness of the oxidation of the pyrite, not only of its iron but of the greater part of the sulphur as well. Pure pyrite itself is attacked with extreme slowness by boiling dilute sulphuric and hydrofluoric acids, either alone or mixed, but the moment a ferric salt is introduced the case is altogether different.

The complete solution to the problem was afforded by the observation made in the Survey laboratory that rocks with hardly an exception and many minerals carry pyrite or pyrrhotite, or both, often in considerable amount, often in traces only. Sulphur can almost always be detected in 2 grams of rock powder.

Experiment has shown (C. c. $\alpha$, p. 138) that with the amounts of sulphides usually found in igneous rocks their effect on the estimation of ferrous iron by the hydrofluoric-acid method at atmospheric: pressure and boiling heat is negligible, though by increasing the amount of sulphide the effect becomes more and more apparent, because of the greater surface of pyrite exposed to the action of the ferric iron of the rock.

$a$ Ber. Deutsch. chem. Gesell., vol. 32, 1899, p. 2217, footnote.

b Ann. Soc. gél. Belgique, vol. 10, 1882-83, p. 101; Zeitschr. anorg. Chemie, vol. 26, 1901, p. 123.

$c$ Bull. U. S. Geol. Survey No. 186, 1901; Am. Jour, Sci., 4th sẹr., vol, 12, 1901, p. 414,

dZeițschr. prakt, Geol., 1899, pp. 250-251, 
Under the conditions of the Mitscherlich method, on the other hand - a temperature of 150 to $200^{\circ}$, and even higher, high pressure, much longer time of action, and impossibility of escape of any hydrogen sulphide that may be formed-the sulphur of the sulphides becomes nearly if not fully oxidized to sulphuric acid at the expense of the ferric iron in the rock, with the production of an equivalent amount of ferrous iron in addition to that resulting from the sulphide itself.

Let us now see what the effect of these traces of sulphides when fully oxidized amounts to. One atom of sulphur (32) requires for its complete conversion to trioxide the oxygen of three molecules of ferric oxide (480), which then become six molecules of ferrous oxide (432). In other words, 0.01 per cent of sulphur may cause the ferrous oxide to appear too high by 0.135 per cent, and 0.10 per cent of sulphur may bring about an error of 1.35 per cent in ferrous oxide. The case is still worse if the sulphur is set free as hydrogen sulphide from a soluble sulphide, for then the above percentages of sulphur produce errors of 0.18 and 1.8 per cent, respectively, in the ferrous oxide determination.

The error caused by sulphides tends to become greater the more there is present of either or both sulphide and ferric salt. Now, the highly ferruginous rocks usually carry more ferric iron than the less ferruginous ones, and they are often relatively high in pyrite and pyrrhotite; hence the increasing discrepancy between the results by the two methods as the iron contents of the rocks rise is fully in accord with the above explanation. ${ }^{a}$

Of course carbonaceous matter will, under the conditions of the Mitscherlich method, likewise reduce sulphuric acid and cause the determination of ferrous iron to be faulty.

Notwithstanding the fact that the Mitscherlich method has thus been discredited in its general applicability to rocks and minerals, it is still probably the best with those which are totally free from sulphides and wholly decomposable. Hence the conditions under which success can best be achieved by it are set forth in the following paragraphs.

\section{B. THE MODIFIED MITSCHERLICH METHOD.}

$\alpha$. Strength of acid.-The method in its original and usual application calls for a mixture of 3 parts of sulphuric acid and 1 of water by weight, or about 3 to 2 by volume, though a still stronger acid is sometimes used. In some cases, however, perhaps in most, much better decomposition of the silicates is effected by reversing the proporions of water and acid, or at any rate by diluting considerably beyond the

a For details of experiments showing the worthlessness of the Mitscherlich method for rocks and minerals which contain even a trace of free sulphur or sulphides, see Hillebrand, W. F., and Stokes, H. N., Relative value of the Mitscherlich and hydrofluoric-acid methods for ferrous-iron determinations: Jour. Am. Chem. Soc., vol. 22, 1900, p. 625; and Zeitschr. anorg. Chemie, vol. 25, 1900, p. 326. 
above proportion. Hereby the separation of salts difficultly soluble in the stronger acid is avoided and the actual solvent effect on the minerals seems to be in no wise diminished.

' $\beta$. Filling, sealing, and heating of the tube.-The very finely powdered mineral having been introduced into a tube of resistant glass free from ferrous iron, the open end is drawn out, so as to leave a funnel for the introduction of the acid. A very little water is then introduced and carefully heated to boiling for a moment to expel all air from the powder. The diluted acid-which has just been boiled down from a state of greater dilution in order to have it free from air-is then poured in until the tube is about three-fourths filled. Carbon dioxide is then introduced, from a generator which has been in active operation for some time, through a narrow glass tube drawn out of the same kind of glass as that of which the decomposing tube consists. In a few moments the air is expelled, and the small tube is then sealed into the large one over the blast lamp without interrupting the gas current until the very last instant, when to prolong it would perhaps cause a blowing out of the softened glass. The interruption of the current at the proper moment is easily effected by the pressure of the thumb and finger holding the small tube at the point where it enters the rubber tube leading from the gas generator. No breakage in the oven ever occurs as a consequence of thus fusing one tube into the other.

The heating is done in a bomb oven at any desired temperature up to, say, $200^{\circ}$, and continued at intervals until examination by aid of a low-power lens shows that decomposition is complete or has progressed as far as can be hoped for. By inclosing the glass in an outer tube of strong steel, properly capped ${ }^{a}$ and containing a little ether or benzine to equalize the pressure on both sides of the glass, the temperature can be elevated far beyond what is otherwise permissible, and the decomposition will then doubtless be more complete with refractory silicates.

$\gamma$. Reason for introducing gas and sealing as above directed.-The usual practise in employing the above method has been to expel air before sealing by introducing a few crystals or lumps of an alkali carbonate or bicarbonate, the gas set free on their contact with the acid being supposed to effectively expel all air. That this is not accomplished the following series of comparative results, long since published elsewhere, ${ }^{b}$ fully shows. The material used was the oxide of uranium $\mathrm{U}_{3} \mathrm{O}_{8}$, requiring by theory 32.07 per cent of $\mathrm{UO}_{2}$. Operating as just described on from 0.3 to 0.5 gram, the results were

$$
31.06,31.07,29.72,29.33,29.89,30.69 \text {, }
$$

$a$ Ullmann, C., Zeitschr. für angew. Chemie, 1893, p. 274; Zeitschr. anal. Chemie, vol. 33, 1894, p. 582.

b Bull. U. S. Geol. Survey No. 78, 1891, p. 50; Chem. News, vol. 64, 1891, p. 232 
:vinereas after filling the tube with gas from a generator there was found

$$
32.11,31.90,32.15,32.12,32.06,32.17,32.28 \text {, }
$$

the average error of the former series being 1.78 per cent. The percentage error would, of course, be reduced by increasing the weight of mineral operated on. An average error equal to the above when employing 1 gram of ferrous minerals would make the percentage for $\mathrm{FeO}$ about 0.3 per cent too low. While the absolute error might be the same in all cases, the relative error would increase with minerals low in ferrous iron.

\section{THE HYDROFLUORIC-ACID METHOD.}

a. PRINCIPLE OF THE METHOD.

This method consists simply in securing complete decomposition of the very fine powder in absence of air by means of hydrofluoric acid in presence of sulphuric acid, and titrating the ferrous iron in the resulting solution. It is the one which has been almost exclusively used. in the Survey laboratories. The form of apparatus hitherto employed is the original one devised by J. P. Cooke ${ }^{a}$ (fig. 20, p. 141). This does not permit driving off the superfluous hydrofluoric acid, as does that of Treadwell (fig. 21, p. 143) to some extent, which latter ought therefore, for a reason developed below, to afford slightly lower and more accurate results, other conditions being equal. But the method as ordinarily carried out is subject to two defects of opposite sign but unequal value, the second and greater of which has remained unsuspected until recently.

It is possible to titrate ferrous iron in presence of sulphuric acid and as much as 5 to $7 \mathrm{~cm} .^{3}$ of 40 per cent hydrofluoric acid in a total volume of 200 to $400 \mathrm{~cm} .^{3}$ almost if not quite as exactly as in sulphuric acid alone, provided the iron solution is diluted with air-free water and the titration is made immediately after adding the hydrofluoric acid and with all possible dispatch. This condition can naturally not be fulfilled in practice because the mineral must be in contact with the acid for a long time in the apparatus commonly employed, and it seems to be almost impossible to prevent some oxidation during this period.

$$
\text { b. DEFECTS OF THE METHOD. }
$$

$\alpha$. Oxidizability of divalent manganese by permanganate in presence of hydrofluoric acid.-Manganic fluoride in dilute solutions is hardly at all dissociated, wherein it differs from manganic sulphate. The entering of the manganic ion as fast as formed into the undissociated state explains the fact that in presence of hydrofluoric acid 
divalent manganese is readily oxidized by permanganate, whereby a sharp end reaction is rendered unattainable in presence of much hydrofluoric acid, say over $7 \mathrm{~cm} .^{3}$ of 40 per cent acid. It is easy to obtain a transitory pink color throughout the liquid, but this rapidly disappears, the more rapidly the greater the amount of hydrofluoric acid or of manganous salt present. The oxidizer can be added by the cubic centimeter to solutions already containing manganous sulphate in presence of hydrofluoric acid without producing a more than passing pink color. The solution, however, takes on in ever increasing intensity the red-brown color characteristic of manganic salts. The decolorization due to this cause is hence much more pronounced in the case of rocks high in ferrous iron than in that of those low in this constituent, because of the greater amount of manganous salt resulting from reduction of a correspondingly larger amount of permanganate, and is also greater with increasing hyddrofluoric acid. In presence of but little ferrous iron, up to, say, 2 centigrams, and 5 to $7 \mathrm{~cm}^{3}$ of hydrofluoric acid the color produced by a drop of permanganate lasts some time, but is very evanescent as the terrous iron, and consequently the manganous salt formed, increases.

E. Deussen, ${ }^{a}$ on the basis of but a few tests, ascribes the extra consumption of permanganate to some obscure action of iron and seeks to counteract it by the addition of manganous sulphate. In both these assumptions he is, however, certainly in error, as shown by the fact that the addition of a drop or two of this oxidizer to a solution of ferric sulphate in even a large amount of hydrofluoric acid produces a very stable coloration, which is destroyed by the addition of manganous sulphate and by the above-mentioned very evident partial reoxidation of the reduced manganese. This last, the oxidation of divalent manganese by permanganate in presence of hydrofluoric acid, can be effected with the greatest ease in entire absence of ferric iron.

Numerous tests have been made to ascertain the error due to oxidation of the manganese under conditions similar in respect to the amounts of iron involved to those encountered in rock analysis. Increasing amounts of hydrofluoric acid were added to fixed amounts of ferrous sulphate and sulphuric acid and the titration made with the least possible expenditure of time. With not more than $7 \mathrm{~cm}^{3}$ of 40 per cent hydrofluoric acid a very slight tendency was observed toward high results, but in most cases the results were identical with those obtained in presence of sulphuric acid alone. As the amount of hydrofluoric acid was increased to $10 \mathrm{~cm} \cdot{ }^{3}$ a perceptible increase was noticed, which became markedly greater with $15 \mathrm{~cm}^{3}$ of the acid, amounting in this case to about $0.2 \mathrm{~cm} .{ }^{3}$ in the average on a normal consumption of $20 \mathrm{~cm}^{3}$ of permanganate of $0.0032 \mathrm{FeO}$ titer. The personal factor enters here, G. Steiger having found slightly greater 
differences, but it is possible that they resulted from his titrating in more concentrated solutions, for with greater dilution the differences are unquestionably less, according to experiments of my own.

Hence, after decomposition of the rock, removal of the excess of hydrofluoric acid is called for if the best results are to be expected. To a certain extent Treadwell's apparatus accomplishes this, but, as the experiments show, its complete expulsion is unnecessary.

$\beta$. Extreme oxidizability of divalent iron by free oxygen in presence of hydrofluoric acid or a fluoride.-In comparing two series of results obtained with the Cooke apparatus, using a solution of ferrous sulphate in sulphuric acid alone in the one and with hydrofluoric acid in addition in the other, a minus error was in all cases observed when the latter acid was present. An observation made by R. Peters, ${ }^{a}$ but not applied by him to the present case, explains this. Ferric fluoride, like the corresponding manganic salt, is almost undissociated in solution, whereas the sulphate undergoes considerable dissociation, and in this state counteracts, to a great extent, the tendency of any ferrous iron present to become oxidized by free oxygen. Hence the slow oxidation of divalent iron by air in sulphuric-acid solution and its very rapid oxidation in presence of hydrofluoric acid. While a sulphuric solution of ferrous sulphate exposed to the air in an open dish will hardly change in strength during an hour, a similar solution to which hydrofluoric acid has been added will suffer a large measure of oxidation in a quarter of the time. The change in titer becomes pronounced after a few minutes.

In the experiments with the Cooke apparatus just referred to, the minus error should amount to not more than 0.1 to $0.2 \mathrm{~cm} .^{3}$ of permanganate on a normal consumption of $20 \mathrm{~cm}^{3}$ or 0.5 to 1 per cent of the iron, but it may be considerably greater if the utmost care is not taken to employ carbon dioxide as free as possible from air and to exclude most carefully the entrance otherwise of air into the apparatus. It will also be greater the higher the concentration, within certain limits, of the solution during titration.

With a view to lessening the error, if possible, the mode of operating was varied by doing away with carbon dioxide, except at the start; shutting it off as soon as the bath was in active ebullition, and transferring the crucible direct from the hot bath to the titration vessel. The results were, if anything, a trifle better. As the employment of either the Cooke or the Treadwell apparatus involves long contact of the mineral with the acids, and as the experiments last mentioned were favorable in their results, it seemed as if the simple method of J. H. Pratt ${ }^{b}$ might be modified in the same sense. He avoided the use of all apparatus other than a capacious platinum crucible fitted with a perfo- 
rated cover for the introduction of carbon dioxide, in which he boiled the mineral powder with sulphuric and hydrofluoric acids, the decomposition being greatly hastened by the active movement and higher temperature and usually completed in five to ten minutes. The modification consists in doing away altogether with carbon dioxide, except at the start, and depending on the steam of the boiling iron solution to exclude air, a modification which Pratt himself tried with rather considerable minus errors, resulting perhaps from his having a smaller crucible at command than the method really demands. With a crucible of $100 \mathrm{~cm} \cdot{ }^{3}$ capacity, the following results with ferrous sulphate show what are the possibilities of the method:

Ferrous-iron determinations by the modijied Pratt method.

[Strength of permanganate $0.0032 \mathrm{FeO}$ per $\mathrm{cm} .{ }^{3}$ ]

\begin{tabular}{|r|c|c||c|c|c|}
\hline $\begin{array}{c}\text { Time of } \\
\text { boiling. }\end{array}$ & $\begin{array}{c}\text { Perman- } \\
\text { ganate } \\
\text { used. }\end{array}$ & $\begin{array}{c}\text { Normal } \\
\text { consump- } \\
\text { tion of per } \\
\text { manganate }\end{array}$ & $\begin{array}{c}\text { Time of } \\
\text { boiling. }\end{array}$ & $\begin{array}{c}\text { Perman- } \\
\text { ganate } \\
\text { used. }\end{array}$ & $\begin{array}{c}\text { Normal } \\
\text { consump- } \\
\text { tion of per- } \\
\text { manganate. }\end{array}$ \\
\hline Minutes. & $c m .3$ & $c m .3$ & Minutes. & $c m .3$ & $c m .3$ \\
10 & 4.9 & 4.8 & 10 & 19.4 & 19.2 \\
10 & 4.8 & 4.8 & 10 & 19.3 & 19.2 \\
10 & 5.0 & 4.8 & 15 & 19.25 & 19.2 \\
10 & 4.9 & 4.8 & 15 & 19.1 & 19.2 \\
10 & 9.6 & 9.6 & 15 & 19.3 & 19.2 \\
10 & 9.6 & 9.6 & 20 & 19.2 & 19.2 \\
10 & 9.6 & 9.6 & 20 & 19.3 & 19.2 \\
10 & 19.2 & 19.2 & & & \\
\hline
\end{tabular}

These results leave little, if anything, to be desired. They show either normal values or a slight plus error instead of the invariable negative one of all previous determinations. The method has not yet been tested on rocks in comparison with that of Cooke, so that it would be premature to assert that this is the one to employ in preference to the other or to that of Treadwell, but the indications are so favorable that extended tests will be carried out in the regular course of Survey work.

c. INFLUENCE OF SUlPHIDES, VANADIUM, AND CARBONACEOUS MATTER ON THE DETERMINATION OF FERROUS IRON BY THE HYDROFLUORIC-ACID METHOD.

A dark color of the undissolved residue may be due to pyrite, graphite, or carbonaceous matter. The first of these affects the result but little, the second probably not at all, and they can be distinguished by their behavior toward nitric acid. Organic matter of course renders impossible the determination of ferrous iron.

$\alpha$. Sulphides.-Pyrite, in the quantities usually met with in igneous rocks, is probably without serious effect on the ferrous-iron determination by any of the hydrofluoric-acid methods. This sulphide is very resistant toward attack in the absonce of oxygen, as is shown by the fact that if present in any quantity it can be readily recognized in the residue after titration. In any case it is impossible to allow for an 
error introduced by its possible decomposition, and the result of titration must count as ferrous iron. In the case of soluble sulphides two sources of error are introduced-that of reduction of ferric iron by hydrogen sulphide evolved, and that due to the ferrous iron which the sulphides themselves may contain, especially if pyrrhotite is present. The first of these is perhaps negligible, since most of the hydrogen sulphide would probably be expelled without reducing iron. The second is approximately measurable if it is known that pyrrhotite is the only soluble sulphide present, and its amount has been ascertained by determining the hydrogen sulphide set free on boiling with hydrochloric acid in a current of carbon dioxide. In this case a correction is to be applied to the result of titration for total ferrous iron (see also 24 . B. b, p. 160, under "Suiphur").

In order to obtain quantitative data regarding the effect of pyrite on the ferrous-iron estimation by the hydrofluoric-acid method the following tests were made: Part of a fine crystal of pyrite was rather finely powdered and boiled with dilute sulphuric acid, which extracted considerable ferrous iron, derived presumably from admixed or intergrown pyrrhotite, since a second boiling with fresh acid gave a negative test for ferrous iron. After washing by decantation with water, followed by alcohol and ether; the powder was dried and further pulverized. A quarter of a gram of it when treated with hydrofluoric and sulphuric acids in a large crucible by the Cooke method for ferrous iron, then rapidly filtered through a very large perforated platinum cone fitted with filter paper, required but 2 drops of a permanganate solution representing only 0.0032 gram $\mathrm{FeO}$ to the cubic centimeter.

Since, however, Dr.H.N. Stokes has found ${ }^{a}$ that the oxidizing effect of ferric salts on pyrite and other sulphides is vastly greater than seems to have been suspected (see p. 132), the following tests were made in order to ascertain the probable error due to this action under the conditions prevailing in rock analysis: Successive portions of 1 gram each of a horn blende schist, free from sulphur and carrying 10.09 per cent $\mathrm{FeO}$ as the mean of several determinations and 4.00 per cent $\mathrm{Fe}_{2} \mathrm{O}_{3}$, were mixed in a large $100 \mathrm{~cm} .{ }^{3}$ platinum crucible with $0.02,0.025$, and 0.10 gram, respectively, of the above purified pyrite powder, and treated with hydrofluoric and sulphuric acids by the Cooke method, the water bath being at boiling heat for one hour. The cooled contents of the crucible were poured into a platinum dish containing water and titrated rapidly nearly to an end. Then, in order to get rid of the pyrite, which would obscure the end reaction by its reducing effect on the permanganate, the solution was filtered as above and in the clear filtrate the titration was carried to completion. The results were 10.02 , 10.16 , and 10.70. Inasmuch as the smallest of these three charges of

$a$ Bull. U. S. Geol. Survey No. 186, 1901; Am. Jour. Sci., 4th ser., vol. 12, 1901, p. 414. 
pyrite was several times greater than what may be considered an unusually high amount for an igneous rock, it is very evident that for all practical purposes the influence of pyrite on the ferrous determination by the Cooke method is negligible. At the same time it is to be borne in mind that with increased content in ferriciron an increased amount of pyrite will be attacked, and that the extent of this attack is influenced by the degree of fineness of the pyrite powder.

$\beta$. Vanadium.- - If vanadium, when present, exists in the trivalent condition, it necessarily affects with an error varying with its amount the result of titration for ferrous iron. If the amount of vanadium is known a correction can be applied as follows: One molecule of $\mathrm{V}_{3} \mathrm{O}_{3}$ (150.8) in oxidizing to $\mathrm{V}_{2} \mathrm{O}_{5}$ requires as much oxygen as four molecules of $\mathrm{FeO}$ (288) when oxidized to $\mathrm{Fe}_{2} \mathrm{O}_{3}$. The proportion, $150.8: 288:$ : $\mathrm{V}_{2} \mathrm{O}_{3}$ present : $x$, therefore gives the figure to be deducted from the uncorrected value for $\mathrm{FeO}$. That this correction is very needful with many of the basic rocks becomes at once evident from the following perhaps extreme example:

Found 2.50 per cent apparent $\mathrm{FeO}$ in a rock containing .13 per cent $\mathrm{V}_{2} \mathrm{O}_{3}$.

Deduct .25 per cent $\mathrm{FeO}$ equivalent in its action on $\mathrm{KMnO}_{4}$ to $.13 \mathrm{~V}_{2} \mathrm{O}_{3}$.

Leaving 2.25 per cent $\mathrm{FeO}$ corrected.

Found 5.00 per cent apparent total iron as $\mathrm{Fe}_{2} \mathrm{O}_{3}$ in the same rock.

Deduct .14 per cent $\mathrm{Fe}_{2} \mathrm{O}_{3}$ corresponding to .13 per cent $\mathrm{V}_{2} \mathrm{O}_{3}$.

Leaving 4.86 per cent corrected total iron as $\mathrm{Fe}_{2} \mathrm{O}_{3}$

Deduct 2.50 per cent $\mathrm{Fe}_{2} \mathrm{O}_{3}$ equivalent to 2.25 per cent $\mathrm{FeO}$.

Leaving 2.36 per cent $\mathrm{Fe}_{2} \mathrm{O}_{3}$ in the rock.

Failure to correct for the vanadium in both cases would have made the figures for $\mathrm{FeO}$ and $\mathrm{Fe}_{2} \mathrm{O}_{3}$, respectively, 2.50 and 2.22 instead of 2.25 and 2.36 as shown above.

$\gamma$. Carbonaceous matter.-As said before (c, p. 138), matter of organic origin other than graphitic carbon renders the results of the ferrous-iron determination altogether unreliable.

\section{d. THE METHOD IN ITS VARIOUS MODIFICATIONS.}

$\alpha$. According to Cooke. ${ }^{a-T h e}$ apparatus, as shown in fig. 20, consists of a small water bath of a single opening and covered with a glass funnel the stem of which has been cut off near the flare, resting in a troughlike depression of the specially made cover. Into this trough water constantly drops from a tubulated bottle, thus securing a perfect water joint and serving to keep the bath full by overflowing on the inside. For the more perfect exclusion of air it is best to use water that has been freshly boiled. Through a small metal pipe carbonic acid gas flows into the bath under the cover, but above the surface of the water, and rising through notches in the edge of the opening of the cover fills the funnel and crucible. (See footnote ${ }^{b}$, p. 91.) 
A gram of the finely ground powder ${ }^{a}$ is placed in a platinum crucible of 80 to $100 \mathrm{~cm}^{3}$ capacity and stirred up therein with water enough to moisten and about $10 \mathrm{~cm}^{3}$ of dilute sulphuric acid (1 acid to 4 to 6 water by volume). If the rock contains carbonates there will be effervescence, hence the acid must be added cautiously and the

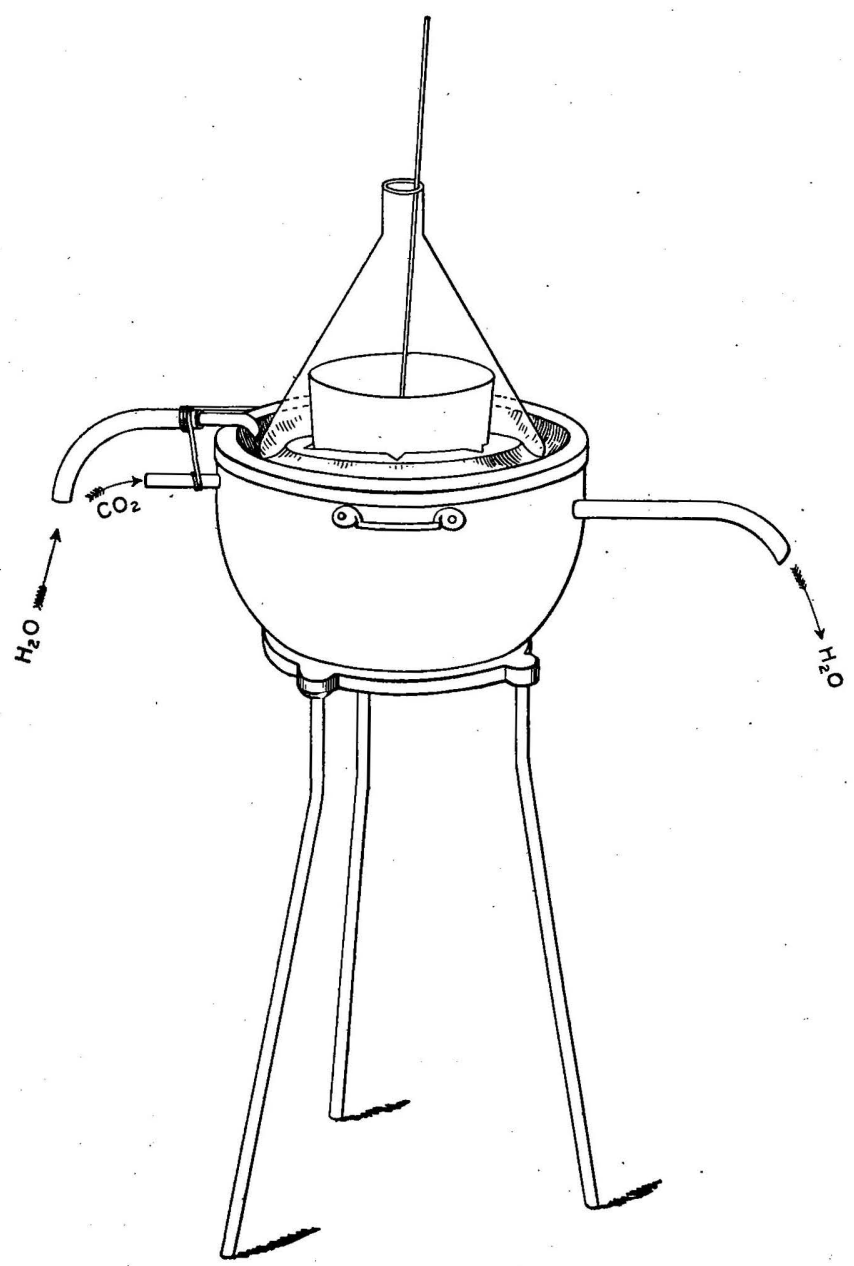

FIG. 20.-Cooke's apparatus for ferrous-iron determination.

cover placed on till action is over. There need be no fear of oxidation of ferrous iron at this stage, should any go into solution. The cover is rinsed if need be and the crucible at once placed in the opening of the bath, the funnel put in place, the gas current started, as also the flow of water from the reservoir, and the lamp under the bath already

$a$ The fineness of grinding will depend on the nature of the rock. Most granitic rocks require but moderately fine grinding, those high in ferruginous and refractory minerals generally, as tourmaline, peed to be very fine: 
full of water is lit. As soon as it can be safely assumed that the air in both funnel and crucible has been displaced a measured volume of strong hydrofluoric acid $\left(5\right.$ to $7 \mathrm{~cm} .^{3}$ ) is poured into the crucible through a funnel of platinum or rubber and a platinum stirring rod is inserted through the stem of the glass funnel into the crucible. In absence of a suitable funnel for the acid, the glass may be momentarily raised for the introduction of the acid, but not before the crucible has become filled with carbon dioxide.

When steam issues strongly from the funnel, the gas current from the generator is stopped and the apparatus left to itself for an hour, except for occasional stirring of the powder. When no gritty matter can longer be felt or when experience tells that decomposition must be complete, the gas current is again turned on, the lamp extinguished, and the water flow increased to the capacity of the outlet tube from the bath. In fifteen minutes the crucible and contents should be cool. The rod is then removed and placed in the titration vessel containing freshly boiled water (usually about $300 \mathrm{~cm}^{3}$ ), which is already under the burette, the contents of the crucible quickly follow with the rinsings, and the permanganate is run in as rapidly as possible with constant but gentle stirring till the first pink blush appears throughout the whole liquid for at least a couple of seconds. With little iron the color will last some time, but with increasing amounts, as also with increasing amounts of hydrofluoric acid, it fades out with ever greater rapidity. Until the operator has become experienced a duplicate determination should always be made. It is to be noted that the crucible is always much fuller at the end of the heating than when first placed on the bath, because of condensation of steam.

Instead of allowing the crucible to cool in a current of carbon dioxide, it may be at once transferred to the titration dish and permanganate run in as above directed. If carefully done, the experiments already referred to seem to show that the results by this variation of the method may be a trifle better than those obtained after cooling.

Those inexperienced with the method in either variation should not fail to test it first with solution of ferrous sulphate that has been standardized without hydrofluoric acid, and in practice duplicate determinations should be made if possible.

$\beta$. According to Pratt (modified).-One gram of the finely ground powder is treated in a capacious crucible, as in $\alpha$, with water enough to moisten and about $10 \mathrm{~cm} .^{3}$ of dilute sulphuric acid, and the crucible with cover on is placed on a triangle well down over a lamp turned low and protected from drafts. The air in the crucible is displaced rapidly by carbon dioxide entering beneath the lid slightly raised on one side. In a few seconds the liquid boils, but before allowing this to happen the gas current is stopped and the well-fitting lid lowered. 
Then from a small platinum crucible a measured quantity of strong hydrofluoric acid (5 to $7 \mathrm{~cm}^{3}$ ) is poured in with one hand, while the other draws the lid a little to one side. A second suffices. The lid is replaced, the flame is increased for a few moments with great caution not to allow boiling over, and the moment steam is seen to issue around the lid it is again lowered to a point that causes steady ebullition without danger of loss. Steam should issue continually for five, ten, or äs many minutes as may be deemed necessary or allowable. Then the crucible, still covered, is transferred with the tongs shown in fig. 1 (p. 28) to the titration dish and the operation is concluded as in $\alpha$. The method should first be tested by the novice with ferroussulphate solution that has been standardized without hydrofluoric acid. It is important not to prolong the boiling unduly, for salts not easily soluble will separate, and as the temperature rises the oxidizing action of the concentrating sulphuric acid may come into play.

The directions given by $\operatorname{Pratt}^{a}$ with reference to the treatment of a possible undissolved residue of very refractory minerals must be understood as applying only to homogeneous minerals and not to rocks, where the relations of ferrous and ferric iron in the undecomposed portion are certainly different from those in the part dissolved.

$\gamma$. According to Treadwell. - The specially ground powder in a dish or capacious crucible is placed, after stirring up

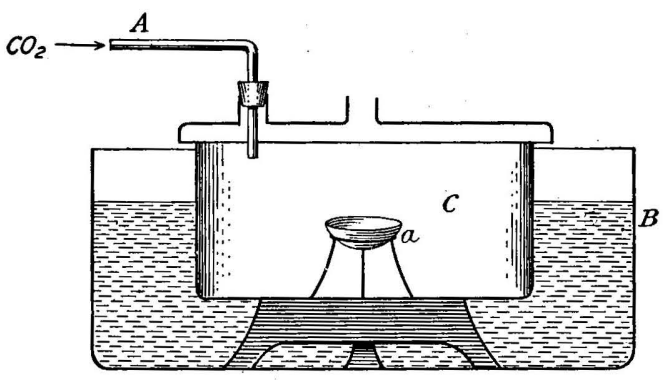

FIG. 21.-Treadwell's apparatus for ferrous-iron determination. B, Paraffin bath; C, lead box with closely fitting cover of same material; $a$, platinum dish resting on glass support.

with 5 or $10 \mathrm{~cm} .{ }^{3}$ of dilute sulphuric acid, on a support of glass or platinum $a$ in the lead box $\mathrm{C}$, which in turn is supported in a paraffin bath B, fig. 21. After covering the box with its leaden cover there is introduced through $A$ a rapid current of carbon dioxide ${ }^{b}$ until the air is displaced. The cover is then quickly raised. Concentrated hydrofluoric acid (5 to $\left.7 \mathrm{~cm}^{3}\right)$ is added to the dish and the cover is replaced without interrupting the current. The contents of the dish are stirred with a platinum rod through the opening directly over the dish. The bath has meanwhile been brought to about $100^{\circ}$, a temperature which is maintained with occasional stirring for from one-half to one hour or more, according to the resisting quality of the powder. Then the temperature is raised to about $120^{\circ}$ for an hour to expel most of the hydrofluoric acid. The flame is then extinguished and the solution allowed to cool as quickly as may be, still in the gas current. When 
cool, the contents of the dish are titrated, as in $\alpha$, in presence of an additional few cubic centimeters of sulphuric acid.

Although the chief object of this method is to remove the excess of hydrofluoric acid and thus avoid one source of error in titration, it may be said, so far as my own limited experience with it goes, that the expulsion is by no means perfect at $120^{\circ}$ and that the long duration of the experiment renders it likely that lower results will be obtained than with the variations of the hydrofluoric-acid method described in $\alpha$ and $\beta$, by reason of greater oxidation of ferrous iron.

\section{UNCERTAINTIES OF THE FERROUS-IRON DETERMINATION.}

From the foregoing it is apparent that, despite the utmost care in practical manipulation, the exact determination of ferrous iron in rocks is one fraught with extraordinary difficulties and uncertainties. Only in absence of decomposable sulphides and carbonaceous matter and when the amount and condition of vanadium are known can the result be regarded as above suspicion.

\section{ALKALIES.}

\section{A. THE J. LAWRENCE SMITH METHOD.}

a. ItS ADVANTAges.

The various methods for getting at the alkalies in insoluble silicates differ more in the mode of attack of the mineral powder and in the immediately subsequent treatment than in the final stages. With very few exceptions, since the early days of the Survey's existence, all alkali determinations have been made by the method of J. Lawrence Smith, ${ }^{a}$ which is far more convenient than and fully as accurate as those in which decomposition is effected by hydrofluoric and sulphuric acids, or by bismuth, lead, or boric oxides. One of its chief advantages is the entire elimination of magnesia at the start:

b. REAGENTS AND APPARATUS.

Decomposition of the powder is effected by heating it with its own weight of ammonium chloride and eight times as much precipitated calcium carbonate.

The ammonium chloride used must be purified, preferably by sublimation, or made by neutralizing pure ammonia by pure hydrochloric acid, and the calcium carbonate is best obtained from pure calcite by solution and reprecipitation. However obtained, this last is rarely free from alkalies, which must be estimated once for all in a blank test in order to apply a correction. Eight grams of the carbonate will yield usually from 0.0012 to 0.0016 gram of alkali chlorides, almost

a Am. Jour. Sci., 2d ser., vol. 50, 1871, p. 269; Am. Chemist, vol. 1, 1871; Annalen Chem. und Pharm., vọl. 159, 1871, p. 82. 
entirely the sodium salt, but the amount has been brought down to half the above by very long washing. This correction may be admitted at once to be a defect of the method, but it is one easily applied with safety. It is not at all certain that all the alkali found in the blank test comes from the carbonate. Much hot water is used for the analysis and a hot-water bottle, even of Jena glass, will afford weighable amounts of alkali.

The ignition may be made in a covered crucible of ordinary shape and of about 20 to $30 \mathrm{~cm} .^{3}$ capacity, heated to dull redness for not more than two-fifths of its height, but the heat has to be kept so low in this case to avoid loss by volatilization that perfect decomposition is not always assured. ${ }^{a}$ Hence, to avoid waste of time in very fine grinding, the form of crucible with cap originally advocated by Smith is very much to be preferred, since it permits, when set at an angle through an opening in the side of a fire-clay cylinder, of the application of the full heat of two burners, and perfect decomposition invariably results without the need of extraordinary care in grinding. The crucible used in this laboratory (fig. 22) for one-half gram of rock powder and 4 grams of calcium carbonate is $8 \mathrm{~cm}$. long, $1.8 \mathrm{~cm}$. wide at the mouth, and $1.5 \mathrm{~cm}$. at the bottom. For double the amounts or more the dimensions are $8 \mathrm{~cm} ., 2.5$ $\mathrm{cm}$, and $2.2 \mathrm{~cm}$. The weights are 25 and 40 grams.

c. TREATMENT OF THE MINERAL POWDER.

Perfectly satisfactory results are to be obtained with but half a gram

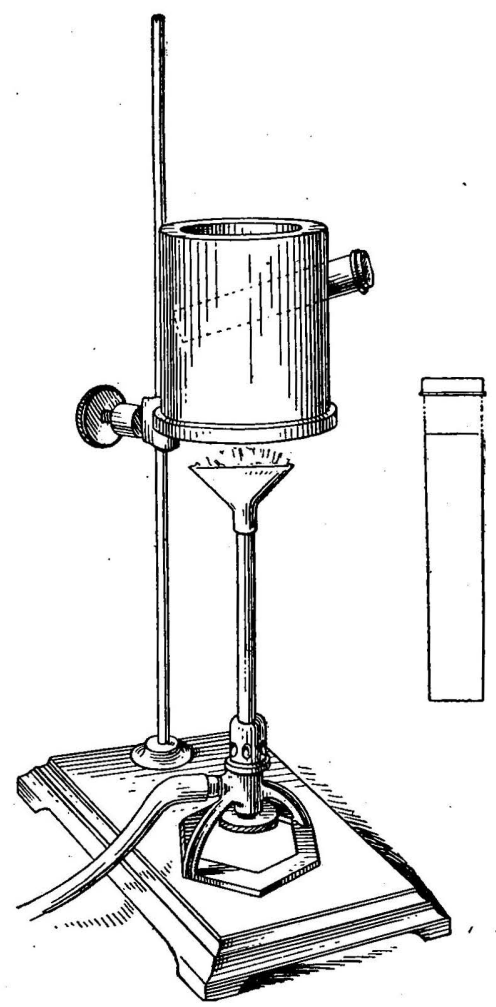

FIG. 22.-The J. Lawrence Smith crucible for alkali determinations. For dimensions see text. of rock powder. This is weighed out, ground down somewhat finer in a large agate mortar, mixed with its own weight of sublimed ammonium chloride, and the two thoroughly ground together. Then nearly all of 4 grams of calcium carbonate is added and the grinding continued till a thorough mixing has resulted. The contents of the mortar are trans-

$a$ R. L. Steinlen (Chem. Zeit., vol. 29, 1905, p. 264) describes a simple cooler for the lid of an ordinary crucible to obviate this difficulty. E. W. Morley (private communication) uses with good effect as a lid a closely fitting small platinum dish filled with cold water.

Bull. 305-07-10 
ferred to the long crucible, the rest of the carbonate being used for rinsing off mortar and pestle. The crucible is then capped and placed in an inclined position in a clay cylinder (fig. 22) or through a hole in a piece of stout asbestos board clamped in a vertical position, and heated for about ten minutes by a low, flat flame placed at considerable distance beneath. As soon as the odor of ammonia is no longer perceptible the nearly full flame of two Bunsen burners is substituted, and continued for forty to fifty minutes. The sintered cake ${ }^{a}$ detaches readily from the crucible as a rule; if not, it is softened up in a few minutes by hot water and digested in a dish until thoroughly disintegrated. It is first washed by decantation, and any lumps are broken up by a pestle, then thrown on the filter and well washed with hot water. The residue should dissolve completely in hydrochloric acid without showing the least trace of unattacked mineral, not even of quartz, though sometimes a few black particles of iron ores will dissolve but slowly.

d. SEPARATION OF CALCIUM AND SULPHURIC ACID.

All but a trifling amount of the calcium is separated at boiling heat in a large platinum dish by double precipitation by ammonia and ammonium carbonate. The combined filtrates are evaporated to dryness and the ammonium salts are carefully driven off (B, p. 30). From the aqueous solution of the residue-but a few cubic centimeters in bulk-the rest of the calcium is thrown out by ammonia and ammonium oxalate, the last being more effective than the carbonate. The filtrate, caught in an untared platinum crucible or small dish, is evaporated to dryness and gently ignited; the residue is moistened with hydrochloric acid to decompose any alkali carbonate that may have been formed, again evaporated, ignited, and weighed. On solution in water a few tenths of a milligram of fixed residue is invariably left, which should be collected, ignited, and weighed in the same crucible or dish in order to arrive at the weight of the chlorides.

1. If the rock contains sulphur this will be in part found with the chlorides as sulphate. Therefore, if the sulphur is at all considerable in amount it must be removed by a drop of barium chloride before the final precipitation of the calcium. The excess of barium is removed by ammonium carbonate and the last of the calcium by ammonium oxalate, as above. If the sulphur is not thus removed there is danger, if not certainty, of the potassium chloroplatinate carrying sodium sulphate. A faint reaction for sulphate can usually be obtained, anyway, if the evaporations have been made on a water bath heated by gas.

$a$ To avoid the formation of a melted cake with silicates high in iron it is advisable to increase the proportion of calcium carbonate. In order to lessen the chance of adhesion to the bottom W. T. Schaller, of this laboratory, finds it well to place a layer of calcium carbonate at the bottom of the crucible before introducing the mixture. Mr. Steiger finds that the slacking of the cake is aided by using but 1 or $2 \mathrm{~cm} .^{3}$ of water at first. 
e. PRECipitation of potassium.

To the solution of the chlorides in a small porcelain ${ }^{a}$ dish an excess of platinic chloride solution is added. The dilution should be such that when heated on the water bath any precipitate that may form wholly redissolves. Evaporation is then carried on till the residue solidifies on cooling. It is then drenched with absolute alcohol ${ }^{b}$ or with that of 80 per cent strength, filtered by decantation through a very small filter and washed by decantation with alcohol of the same strength. The precipitate is not brought onto the filter more than can be avoided. Dish and filter are then dried for a few minutes to remove adhering alcohol, the contents of the former are transferred to a weighed platinum crucible or very small dish, and what still adheres to the porcelain is washed through the filter with hot water into the weighed receptacle. This is now placed on the steam bath and afterwards heated for a short time to $135^{\circ}$ in an air bath. It is very important to cover the dish at first in the air bath, for decrepitation with resultant loss sometimes takes place if this is not done. With rocks low in potash drying at $100^{\circ}$ suffices fully to expel all water. The factor used for reduction of $\mathrm{K}_{2} \mathrm{PtCl}_{6}$ to $2 \mathrm{KCl}$ is 0.307 and of $2 \mathrm{KCl}$ to $\mathrm{K}_{2} \mathrm{O}, 0.632$. It does not seem necessary with the relatively small amounts of potassium in rocks to make use in place of the first of these of the slightly different empirical factor given in some textbooks, a factor based on the analysis of commercial potassium salts. If thought desirable the potassium salts can be tested spectroscopically for cæsium and rubidium.

\section{f. SODIUM AND LITHIUM.}

If it is desired to check the sodium, ordinarily determined by difference, this can readily be done by evaporation of the alcoholic filtrate from the potassium chloroplatinate, reduction of the platinum by hydrogen or otherwise as mentioned in the footnote, ${ }^{c}$ filtration, evaporation, and weighing of the sodium chloride. A check made in this way should agree with the determination by difference within half a milligram on the weight of the chloride. Lithium may then be tested for by the spectroscope, or in case no direct weighing of the sodium chloride is made, the evaporated alcoholic solution may be examined directly. Lithium is almost invariably

\footnotetext{
$a$ Preferred to platinum because of the possibility, under certain rare and ill-understood conditions, of the formation of an insoluble platinous compound, probably by reaction between the platinum of the dish and that of the salt. (See also Bolm, F., Zeitschr. anal. Chemie, vol. 38, 1899, p. 349).

$b$ H. Precht (Zeitschr. anal. Chemie, vol. 18, 1879, p. 513,) claims that this is to be preferred to 80 per cent alcohol, especially if evaporation has been carried to dehydration of the sodium salt. A. Atterberg disputes this final statement and says that 80 per cent alcohol gives better results.

$c$ When haste is not an object, this way of Bunsen's for removing platinum from the chlorides of the alkalies is by far the neatest and most satisfactory. The small flask containing the solution is placed in a water bath and attached to a hydrogen generator. After expelling all air the flask is closed, without breaking connection with the generator, and left to itself, except for occasional light shaking up, till reduction is accomplished. A more expeditious and very satisfactory reduction is effected by evaporating the solution to dryness with metallic mercury, then heating to expulsion of the excess of mercury and of its chloride (Sonstadt, E., Jour. Chem Soc., vol. 67, 1895, p. 984, who thus reduces potassium chloroplatinate in order to weigh its platinum).
} 
present, but almost never in amount to warrant quantitative estimation. Should it be so, however, the very excellent Gooch methor (summarized below) of separation by amyl alcohol is to be followed. In rock analysis there need be no fear of enough lithium falling with the potassium to cause any concern.

If ammonium carbonate alone has been relied on to separate all calcium (see d., p. 146) the few tenths of a milligram of calcium chloride that escaped precipitation should now be separated from the sodium and the proper correction made.

$\alpha$. Gooch's method a for separating lithium.-To the concentrated solution of the chlorides amyl alcohol is added and heat is applied, gently at first, to avoid danger of bumping, unt il the water disappearing from solution and the point of ebullition rising and becoming constant for some minutes at a temperature which is approximately that at which the alcohol boils by itself, the chlorides of sodium and potassium are deposited and lithium chloride is dehydrated and taken into solution. At this stage in the operation the liquid is cooled and a drop or two of strong hydrochloric acid added to reconvert traces of lithium hydrate in the deposit, and the boiling continued until the alcohol is again free from water. If the amount of lithium chloride present is small, it will now be found in solution and the chlorides of sodium and potassium will be in the residue, excepting the traces, for which correction will be made subsequently. If, however, the weight of lithium chloride present exceeds 10 or 20 milligrams, it is advisable at this point; though not absolutely essential to the attainment of fairly correct results, to decant the liquid from the residue, wash the latter a little with anhydrous amyl alcohol, dissolve in a few drops of water, and repeat the separation by boiling again in amyl alcohol. For washing, amyl alcohol, previously dehydrated by boilingr, is to be used, and the filtrates are to be measured apart from the washings. In filtering it is best to make use of the perforated crucible and asbestos felt, and apply gentle pressurc. The crucible and residue are ready for the balance after drying for a few minutes directly over a flame turned low. The weight of insoluble chlorides actually obtained in this manner is to be corrected by the addition of 0.00041 gram for every $10 \mathrm{~cm} \cdot{ }^{3}$ of amyl alcohol in the filtrate, exclusive of washings, if the insoluble salt is entirely sodium chloride, 0.00051 gram for every $10 \mathrm{~cm} .^{3}$ if potassium chloride constitutes the residue, and, if both sodium and potassium chlorides are present, $0.00092 \mathrm{gram}$; but $* * *$ the entire correction may in any case be kept within very narrow limits if due care be given to the reduction of the volume of residual alcohol before filtration. The filtrate and washings are evaporated to dryness, treated with sulphuric acid, the excess of the latter driven off, and the residue ignited to fusion and weighed. From the weight thus found the subtraction of 0.00050 gram is to be made if sodium chloride constitutes the precipitate, 0.00059 gram if potassium chloride alone is present in the residue, and 0.00109 gram if both these chlorides are present, for every $10 \mathrm{~cm} .^{3}$ of filtrate, exclusive of washings.

Amyl alcohol is not costly, the manipulations of the process are easy, and the only objectionable feature-the development of the fumes of amyl alcohol-is one which is insignificant when good ventilation is available.

The process has been used for some months frequently and successfully, by others as well as by myself, for the estimation of lithium in waters and minerals.

\section{B. SEPARATION OF ALKALIES BY OTHER METHODS.}

When, as may happen in rare instances, it is necessary to estimate alkalies in the main portion after elimination of silica, alumina, lime, etc., in one of the usual ways, the question of a suitable method for separating magnesium becomes important.

a Proc. Am Acad. Arts Sci., 1886, p. 177, Bull. U. S. Geol. Survey No. 42, 1887, pp. 85-86; Chem. News, vol. 55,1887 , pp. $18,29,40,56,78$; Am. Chem. Jour., vol. 9,1887 , p. 33. 
a. THE MERCURIC-OXIDE METIOD.

The old barium-hydroxide method is not to be recommended. The mercuric-oxide method of Zimmermann, whereby the magnesia is precipitated from solution of the chlorides by moist, freshly precipitated, and alkali-free mercuric oxide, can give satisfactory results. The oxide is added in excess to the solution in a platinum crucible and evaporated to dryness. Then the mercuric chloride and most or all of the excess of oxide are expelled by cautious heating. On leaching with water the magnesia remains on the filter. With more than 1 per cent of magnesia the operation must be repeated (Dittrich).

\section{b. THE AMMONIUM-CARBONATE METHOD.}

Lately the once favored method of precipitating the magnesium by neutral ammonium carbonate in concentrated solution has been again recommended. ${ }^{a}$ The magnesium solution must be as strongly concentrated as possible, and a great excess of ammonium carbonate solution must be used. A voluminous precipitate forms, which dissolves on vigorous stirring if enough of the precipitant was used. After a time a crystalline precipitate falls - a double carbonate of magnesium and ammonium-which is insoluble in the concentrated solution of ammonium carbonate. Allow to stand for six to twentyfour hours. Wash with the concentrated ammonium carbonate solution. It is no exercise of undue caution to redissolve and reprecipitate, to make sure of getting all alkali in the filtrate, especially potassium.

c. THE AMYL-ALCOHOL METHOD.

Under certain circumstances, notably absence of lithium, the method of Gooch developed by: Riggs ${ }^{b}$ may be satisfactory. It is similar to that of Gooch for separating lithium from sodium and potassium chlorides by amyl alcohol, and involves the same solubility corrections for the alkali chlorides above noted (f. $\alpha$, p. 148) in the description of Gooch's method. Riggs's summary is as follows:

Evaporate the solution nearly or quite to dryness. Dissolve the residue in as little water as possible and add a few drops of hydrochloric acid. Then add 30 to $40 \mathrm{~cm} .^{3}$ of amyl alcohol and expel the water by bringing the alcohol to boiling. Continue the boiling until the volume of the solution is reduced to $10 \mathrm{~cm} .^{3}$, or even considerably less. In filtering, it is of great advantage to use a perforated crucible and an asbestos felt and to filter under pressure. In case the total chlorides exceed 0.2 gram it may be advisable to decant the liquid, wash the residue, redissolve, and repeat the precipitation. If this be not done, the precipitate should be redissolved with the least possible quantity of water, a few drops of hydrochloric acid added, and the precipitation repeated in the original solution. The filtrate is transferred to a weighed platinum dish and evaporated. Water is added before the alcohol has been expelled, and the evaporation continued. The residue is dissolved

$a$ Wülfing, E. A., Ber. Deutsch. chem. Gesell, vol. 32, 1899, p. 2214. The neutral carbonate is prepared by dissolving 230 grams of ammonium carbonate in $180 \mathrm{~cm}^{3}$ of ammonia of 0.92 specific gravity and enough water to make 1 liter. This is sometimes called Schaffgotsch's solution.

bAm. Jour. Sci., 3d ser., vol. 44 1892, p. 103. 
in water. Sulphuric acid is added in slight excess. This solution is evaporated to dryness, the residue ignited and weighed, and the treatment with sulphuric acid is repeated. Th" residue of insoluble chlorides may be transferred to the weighed perforated crucible and dried at a temperature below their melting points, or it may be dissolved and the solution transferred to a weighed platinum dish, evaporated, and the residue dried as above and weighed.

As with the Gooch method for lithium, the numerous test results are good.

\section{CARBON DIOXIDE, CARBON.}

\section{A. QUALITATIVE TEST FOR CARBON DIOXIDE.}

In the preliminary qualitative test for carbon dioxide, it must be remembered that while calcite gives off its carbon dioxide on treatment with cold acid, dolomite and siderite do not, and hence warming should not be omitted; otherwise, a few tenths per cent of carbon dioxide can very well be overlooked. Moreover, the powder should first be agitated with a little hot water, to remove all entangled air which might otherwise be mistaken for carbon dioxide. In order not to overlook traces the test is best made in a test tube, first boiling the powder with a little water, then cooling and adding dilute hydrochloric acid. If effervescence is immediate the presence of calcite is assured; if escape of bubbles takes place only on warming the carbonate is not calcite. In order to make sure of the reaction it may be necessary to use a pocket lens, holding the test tube in an inclined position and looking down in order better to see the minute bubbles as they stream up along the upper glass wall. It is, of course, important not to mistake escaping hydrogen sulphide for carbon dioxide.

\section{B. QUANTITATIVE TESTS FOR CARBON DIOXIDE.}

a. DIRECT METHOD.

For the quantitative determination of carbon dioxide an apparatus permanently set up is used, of which several forms have been described by different writers. ${ }^{a}$ The one depicted in fig. 23 is a compact arrangement at present used in the Survey laboratory.

The rock powder (1 to 5 grams) is boiled with dilute hydrochloric acid in a small Erlenmeyer flask attached to an upward-inclined condenser, whence, after passing through a drying system - calcium chloride, anhydrous copper sulphate to retain hydrogen sulphide from decomposable sulphides and any hydrochloric acid that may pass over, then calcium chloride again - the carbon dioxide is caught by absorption tubes filled with soda lime followed by calcium chloride. Of course arrangement is made for a current of $\mathrm{CO}_{2}$-free air with which

a For a simple and convenient form, see Treadwell's Quantitative Analysis, American edition, p. 298. 
to sweep out the apparatus before and after the experiment, and for a slow current during its continuance. The results are very accurate and the determination can be quickly carried out.

The manipulations are as follows: Hot water is poured upon the powder in the flask; this is attached to the condenser, and a current of $\mathrm{CO}_{2}$-free air is forced through the whole system, except the weighed

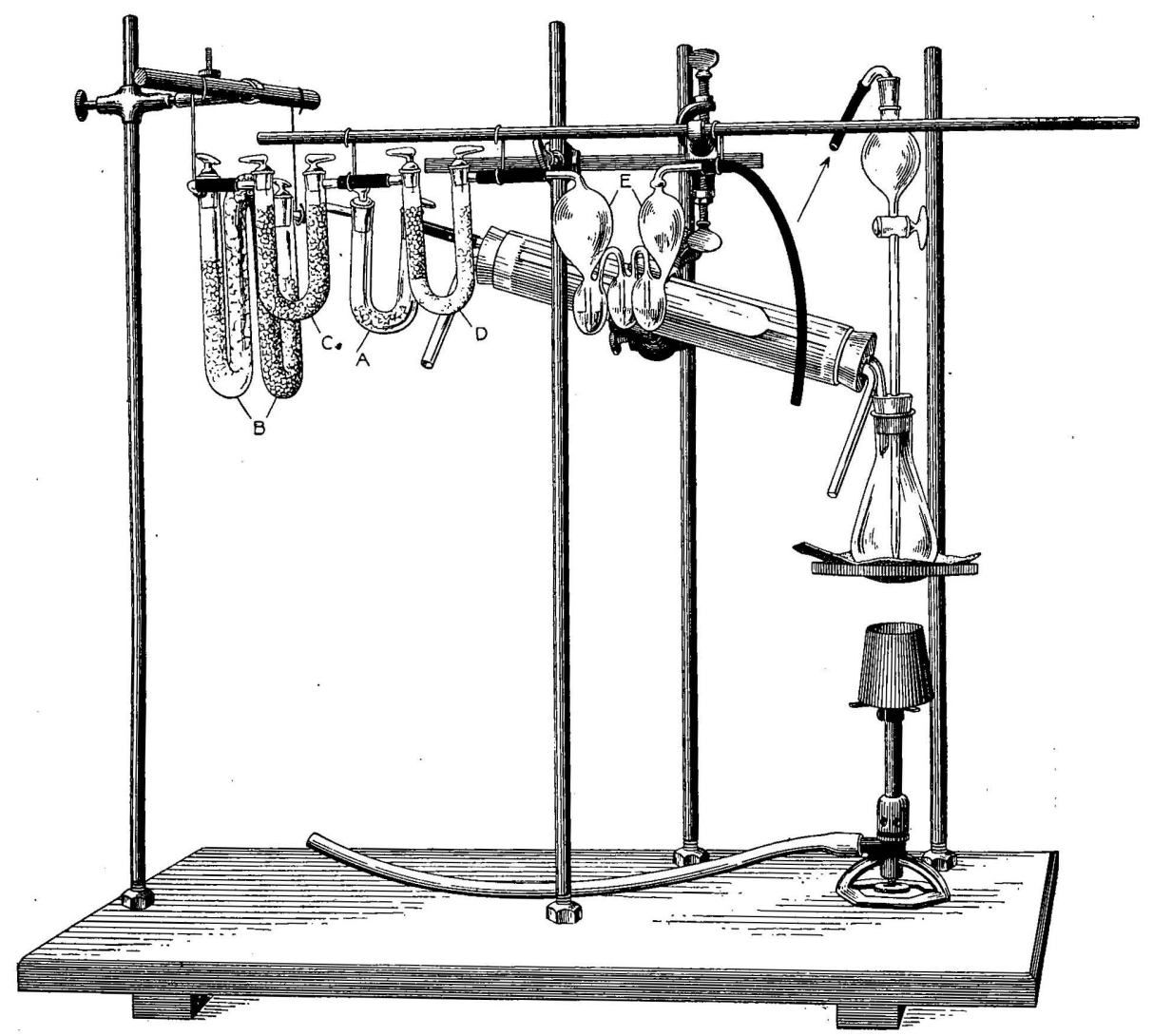

FIG. 23.-Compact form of apparatus for determination of carbon dioxide. A, Protective calciumchloride tube, frequently refilled, to retain most of the moisture from the condenser. $B, D$ ouble $U$ tube, filled in the center with pumice impregnated with anhydrous copper sulphate and at both ends with calcium chloride. $\mathrm{C}$ and $\mathrm{D}$, absorption tubes, $\mathrm{C}$ and one-third of $\mathrm{D}$ containing soda lime, followed in $\mathrm{D}$ by calcium chloride. $\mathrm{C}$ needs to be refilled before the soda lime in $\mathrm{D}$ has absorbed much if any carbon dioxide, $\mathrm{D}$ then requires only occasional refilling, but it will not do to use it indefinitely, since the calcium chloride in it becomes less and less effective as it takes up the water set free from the soda lime in $\mathrm{C}$ during its conversion to carbonate. $\mathrm{E}$, Observation bulbs containing sulphuric acid to show the rate of gas flow.

absorption tubes, until the original air has been displaced, the observation bulbs $\mathrm{E}$ being attached directly to the drying system, $\mathrm{B}$. The stopcock in the separatory funnel is then closed, the latter half filled with hydrochloric acid $(1: 1)$, the rubber stopper of the funnel replaced, the absorption tubes inserted between $\mathrm{B}$ and $\mathrm{E}$, and the acid allowed to flow into the flask, slowly if there is much carbon dioxide, 
rapidly if there is but little. When effervescence diminishes in the former case, at once in the latter, the lamp is lighted and the flow of water through the condenser started. The flame is kept low, so as to secure steady but quiet ebullition, and the gas current is not interrupted, although reduced to a slow rate. With much carbon dioxide the rate of absorption is very readily noted by holding the hand to the soda-lime tubes, which become hot or warm where absorption is taking place. A sufficient time having elapsed, the flame is extinguished and the air current increased. When cool the tubes $\mathrm{C}$ and $\mathrm{D}$ are weighed after remaining some time in the balance case.

The soda lime must be porous, not hard and unabsorptive like that sometimes used for the combustion of nitrogenous organic substances.

For the simultaneous determination of carbonates and the carbon of carbonaceous matter see C., page 152 .

It has already been shown under "Water" ( 5 C. c, p. 66) how in case of need, as paucity of material, the determination of carbon dioxide can be combined with that of water by fusion with lead chromate. Soda-lime absorption tubes then follow the calcium-chloride tube in carrying out the determinations there referred to.

\section{b. INDIRECT METHOD.}

The older forms of apparatus for the indirect determination of carbon dioxide are too well known to need description. Some are fragile or cumbersome and great care is requisite to the attainment of good results. The form recently devised by J. L. Kreider ${ }^{a}$ (fig. 24) has the merit of simplicity, cheapness, and ease of manipulation. It is adapted for determining not only carbon dioxide, but also other volatile substances produced by a variety of reactions, as hydrogen resulting from the action of an acid on certain metals, nitrogen from urea, ammonium oxalate or ammonium chloride by the action of sodium hypobromite, etc. Kreider's test results are excellent, though with carbonates generally low to the extent of about 0.06 to 0.1 per cent.

FIG. 24.-Kreider's apparatus for indirect determination of volatile substances.

The apparatus *** is light and easily made from three test tubes. * * * The test tube A is changed in no way from its original form; $B$ is perforated in the bottom with a hole about $1 \mathrm{~cm}$. in diameter and fits tightly within $\mathrm{A}$; and $\mathrm{C}$, so selected that it fits loosely within $\mathrm{B}$, is drawn out to a small capillary tube.

When the apparatus is to be used, the capillary of $\mathrm{C}$ is pushed through the hole of $\mathrm{B}$, packed loosely with cotton; $\mathrm{B}$ is filled to the depth of from 6 to $8 \mathrm{~cm}$. (about two-thirds of its contents) with granular calcium chloride, and $\mathrm{B}$ and $\mathrm{C}$ are adjusted as shown.

To the test tube, $\mathrm{C}$, is fitted a one-holed stopper, through which passes a short glass tube

a Am. Jour. Sci., 4th ser., vol. 19, 1905, p. 188; Zeitschr. anorg. Chemie, vol. 44, 1905, p. 154. 
which is closed by a rubber cap and plug. Upon removing the plug and applying suction to the short tube the reagent employed to liberate the volatile product to be determined is drawn up through this capillary until $\mathrm{C}$ is sufficiently filled. Upon replacing the plug the reagent remains within $\mathrm{C}$, held by atmospheric pressure.

The tubes A and B may be so selected that very little of the product evolved can escape between them, but in case they fit very loosely a ring of paraffin melted into the mouth of $\mathrm{A}$, about $\mathrm{B}$, by means of a hot iron or wire, seals the joint securely. A very convenient way to attach the paraffin is to melt it between $A$ and another tube, which fits $A$ as does $\mathrm{B}$ and may be removed by a turning motion, leaving the ring into which $\mathrm{B}$ will fit, and which then requires very little heating to make a tight joint. If care be used in taking apart $\mathrm{A}$ and $\mathrm{B}$ at the close of an experiment, such a ring of paraffin remains in place and may be used many times without replacement, being remelted by a touch of the hot wire before every new experiment.

In making the determination the substance under examination is weighed and placed in the bottom of $\mathrm{A}$. The reagent to be employed, 10 to $15 \mathrm{~cm} .^{3}$, is drawn into $\mathrm{C}$ and beld there in the manner described. The test tube $\mathrm{A}$ is slipped over $\mathrm{B}$ and this joint is sealed with paraffin, as has been shown. The apparatus is wiped, placed on the balance, and weighed.

Upon removing the cap from the small tube in $\mathrm{C}$ the reagent runs from $\mathrm{C}$ into $\mathrm{A}$. The volatile product is formed, is forced upward through the drying column of calcium chloride, and escapes through the annular space between B and C. When the action ceases a current of dry air is forced through $\mathrm{C}$ to drive all the volatile products from the apparatus, the cap is then replaced, and the whole placed on the balance to be weighed. The loss in weight represents the volatile product.

\section{CARBON.}

Fusion with lead chromate is commonly resorted to in order to determine the carbon of graphite or carbonaceous matter in rocks and ores, though boiling in presence of sulphuric and chromic acids can be made to yield good results.

If carbonates are at the same time present they can be determined as in B (p. 150), and by a second determination, as just referred to, the total carbon in both forms can be found as $\mathrm{CO}_{2}$, whence that of the graphite is derived by difference. Or, according to G. T. Morgan, ${ }^{a}$ the carbonates are first decomposed as in B by orthophosphoric instead of hydrochloric acid, whereupon chromic acid is added and the carbon oxidized and determined as $\mathrm{CO}_{2}$.

22. CHLORINE.

\section{A. CONDITION IN ROCKS.}

Chlorine may exist in rocks in a water-soluble condition, in minerals that are decomposed by nitric acid, and in those not attacked by this acid. In the first form it doubtless exists as infiltrated sodium chloride or as original inclusions in one or more of the constituent minerals; in the second in minerals of the sodalite group and sometimes apatite; in the last chiefly in the scapolites. Where 
a qualitative test shows the water-soluble form to be present in determinable amount, it must be separately extracted and the amount found deducted from the total as determined in a second portion. Sometimes it may be possible to use the extracted material for the determination of the rest of the chlorine.

\section{B. DETERMINATION OF WATER-SOLUBLE CHLORINE.}

A suitable amount, which may be several grams, of the powder is extracted with water. The filtrate is very often turbid, a condition which may sometimes be prevented by using double filters or by adding some chlorine-free salt, like sodium nitrate, to the wash water. If the filtrate remains persistently cloudy the precipitation of silver chloride may be proceeded with as usual, after acidifying with nitric acid. It is best to let the precipitate settle over night in order to obtain a clear filtrate on the morrow. It is collected on a small filter, washed with water acidified with nitric acid, dried, and ignited in a very small porcelain crucible without allowing the paper to burst into flame. Most of the chloride will be reduced to metal. On disappearance of all carbon a drop of nitric acid is added and evaporated, then similarly a drop of hydrochloric acid, and the crucible gently heated, but not to fusion of the silver chloride, and weighed. The chloride is then dissolved in a few drops of warm ammonia water and separated by filtration from the siliceous matter, which latter is ignited and weighed in order to get the weight of the chloride.

This method of procedure is permissible even when the original aqueous extract was quite clear, though the operation then stops with the weighing of the chloride. Or, if very small in quantity, the dry paper with its contents is wound up in a tared platinum wire and carefully ignited after Bunsen's manner. The increased weight of the wire is due to the metallic silver of the chloride which has alloyed with it.

\section{c. Determination of ACID-SOluble Chlorine.}

a. BY NITRIC ACID.

If nitric acid will decompose the chlorine-bearing minerals, or if it is desired to distinguish between the chlorine in soluble and in insoluble minerals, the rock powder may be boiled for a few minutes with dilute chlorine-free nitric acid. The acid should be very dilute in order to cause no loss of chlorine and the boiling should be as brief as possible for the same reason, and also to prevent gelatinization of the silica from soluble silicates. Acid as dilute as 1:40 will readily decompose apatite and probably the minerals of the sodalite group, but it is doubtful if anything like this dilution is called for. 
The filtrate does not require evaporation for the removal of dissolved silica, but may be precipitated at once with silver nitrate. The precipitate is treated as in $\mathrm{B}$, or if at all appreciable in amount may be collected on a Gooch crucible.

b. BY NITRIC AND HYDROFLUORIC ACIDS.

In many cases in order to obtain all the chlorine it is sufficient to attack the powder by chlorine-free hydrofluoric and nitric acids in the cold, with occasional stirring, and after filtering through paper fitted into a rubber funnel or large platinum cone to throw down the chlorine by silver nitrate. The presence of nitric acid is necessary, since in its absence ferrous fluoride reduces silver nitrate with deposition of crystallized silver. It may be advisable to redissolve the chloride on the filter in ammonia and to reprecipitate by nitric acid and a drop of silver nitrate. It seems difficult to obtain in the market hydrofluoric acid free from a trace of hydrochloric.

\section{DETERMINATION OF CHLORINE BY ALKALI FUSION.}

Chiefly for the last reason, in order to make sure of getting all the chlorine, it is best to fuse with chlorine-free sodium-potassium carbonate, or even sodium carbonate alone, first over the full burner, then for a moment or two over the blast, to leach with water, acidify in the cold with nitric acid, and precipitate by silver nitrate without preliminary separation of silica. After acidification in the cold, except in a very concentrated solution, no precipitation of silica need be feared. The treatment of the precipitate will be as in B and C, according to its amount and freedom from contamination.

\section{FLUORINE (SILICA IN PRESENCE OF FLUORINE.)}

\section{A. IMPERFECTION OF THE QUALITATIVE TEST FOR FLUORINE.}

There is no qualitative test which will reveal with certainty the presence of fluorine in rocks. Heating the powder before the blowpipe with sodium metaphosphate on a piece of curved platinum foil inserted into one end of a glass tube, or in a bulb tube, is not to be relied on in all cases. While as little as 0.1 per cent of fluorine can sometimes be thus detected with ease, much larger amounts in another class of rocks may fail to show.

\section{B. QUANTITATIVE DETERMination OF FLUORINE.}

a. THE METHOD.

For the reason that the fluorine minerals occurring in rocks are mostly attacked but partially, if at all, by strong hot sulphuric acid, none of the methods depending on the volatilization of silicon fluoride can be used. There is the further reason that even with decomposable 
fluorides it is impossible to obtain quantitative results in presence of amorphous silica or silicates. ${ }^{a}$ Only with quartz powder is volatilization perfect. The method to follow is that of Berzelius, which, though not difficult, requires great attention to details and even then affords low results (see $\mathrm{c}$ for causes of error). It admits of the determination of silica at the same time, and is in fact the only method available for silica in fluorides.

Two grams of the rock powder are fused with four to five parts of sodium-potassium carbonate free from fluorine, avoiding the use of a blast if possible. For minerals rich in fluorine and low in silica it may be necessary to add pure silica before fusing in order to effect complete decomposition of the fluoride, just as with the alkaline-earth phosphates. But this will probably never be required in ordinary rock analysis. To the aqueous extract, containing the fluorine and usually much of the silica besides other bodies, several grams of ammonium carbonate are added, the liquid is digested at a gentle heat $\left(40^{\circ}\right)$ for some time, and on cooling more carbonate is introduced. ${ }^{b}$ After twelve hours the precipitate is collected and washed with water containing ammonium carbonate. From the filtrate the excess of the latter is expelled by evaporating nearly to dryness and the somewhat diluted solution is brought toward neutrality as follows, according to the directions of Treadwell. A few drops of phenolphthalein are added, then nitric acid (not hydrochloric in this case, see below) till the red color disappears. The solution is now boiled, the color reappearing, to be again discharged by acid on cooling. These operations are repeated till it requires but 1 to $1 \frac{1}{2} \mathrm{~cm} \cdot{ }^{3}$ of $\frac{2}{\mathrm{~N}}$ acid to discharge the color. There is now added 1 to $2 \mathrm{~cm}^{3}$ of an ammoniacal solution of zinc oxide, ${ }^{c}$ and the liquid is boiled till the ammonia is wholly expelled. The precipitate, containing the last of the silica and some phosphorus, in addition to that thrown down with alumina by ammonium carbonate, is separated and washed with water.

The above-prescribed use of nitric instead of hydrochloric acid for neutralizing is necessitated by the fact that phosphorus, which is

\footnotetext{
a See Daniel, K.,'Zeitschr. anorg. Chemie, vol. 38, 1904, pp. 257-306.

$b$ The use of ammonium nitrate or chloride, instead of carbonate, for throwing out the silica and alumina is not to be recommended because of loss of fluorine on subsequent evaporation (H. Rose).

$c$ Made by precipitating pure zinc-chloride solution with potassium hydroxide, filtering, washing and dissolving the precipitate in ammonia. If the rocks are very basic, it may happen that the amount of silica in the alkaline solution of the fusion is so small that ammonium carbonate may be dispensed with and the zinc-oxide solution added at once, after neutralizing as above.

Instead of the ammoniacal zinc-oxide solution F. Seemann (Zeitschr. anal. Chemie, vol. 44, 1905, p. 343), advocates using the Schaffgotsch solution (p. 149, footnote) in which has been dissorved in the cold 20 grams of freshly precipitated mercuric oxide to the liter. For every 0.2 gram of dissolved sulica 100 $\mathrm{cm} .^{2}$ of this reagent are to be used. The solution is then to be evaporated to complete dryness, the residue taken up with water, filtered and washed, the filtrate again neutralized with hydrochloric acid [nitric in rock analysis-W.F. H.], and again evaporated to remove the last of the silica. Where silica is to be determined at this stage this reagent has the advantage over the zine solution that direct ignition of the precipitates (under a good draft nood) yields at once silica. Its use is manifestly allowable in rock analysis only after the alumina has first been separated by ammonium carbonate.
} 
almost invariably present, and chromium must still be removed, and this can be done only from a nitric solution, as follows: To the still alkaline solution silver nitrate is added in excess, whereby phosphate, chromate, chloride if chlorine is present, and carbonate of silver are precipitated. The last serves to correct any acidity resulting from the reaction between the alkaline phosphate and the silver salt, thus producing the neutral solution needed for complete precipitation of the phosphate and chromate. After slight heating and filtering, the excess of silver is removed by sodium chloride and $1 \mathrm{~cm} \cdot{ }^{3}$ of $\frac{2}{\mathrm{~N}}$ sodiumcarbonate solution is added to the filtrate, which is then boiled in a dish of good size with a large excess of calcium-chloride solution. At this stage there must be no ammoniacal salts in solution, otherwise calcium fluoride may be held up. The precipitate consists of a mixture of calcium carbonate and fluoride, the former serving to aid filtration of the latter. The precipitate is collected on paper and washed with hot water, dried, and ignited separately from the filter as far as possible. If the dish used for the precipitation was of platinum it is not necessary to cleanse it from adhering precipitate, but the contents of the filter are washed back into the dish, the liquid in this is evaporated away, the ash of the filter added, and the dish heated over a free flame to faint redness for a few moments.

Dilute acetic acid is now poured upon the thus gently ignited mixture in small quantities at a time and in amount slightly in excess of that needed to dissolve the calcium carbonate. When visible action ceases the liquid is evaporated to dryness and the residue taken up with hot water and a drop of acetic acid, filtered, dried, and gently ignited.

The amount of fluoride obtained from rocks being usually very small, a single treatment as above suffices, but for the larger amounts obtained from fluorine minerals a second and even third treatment with acetic acid may be needed, filtering and igniting after each. S. L. Penfield and J. C. Minor ${ }^{a}$ found in such cases that if a great excess of acetic acid is used at the start the results are lower than by the repeated treatment. For considerable amounts, when the loss between two treatments is reduced to not more than half a milligram, F. P. Treadwell and A. A. Koch ${ }^{b}$ take the next to the last weight as correct.

b. TESTING OF THE CALCIUM FLUORIDE.

The well-washed and gently ignited calcium fluoride finally obtained in the course of this method should be converted to sulphate is a check on its purity, and at the same time as a qualitative test to ascertain if it really is calcium fluoride by the characteristic odor of the gas given off. Should fluorine be found, and the weight of sulphate not

a Am. Jour. Sci., 3d ser., vol. 47, 1894, p. $389 . \quad b$ Zeitschr. anal. Chemie, vol. 43, 1904, p. 469. 
correspond to that of the fluoride, the former should be dissolved in hot nitric acid and tested for phosphorus by ammonium molybdate solution. If phosphate is absent the impurity may have been silica or calcium silicate- - which of these it would be difficult to decide. In the former case the fluorine might be safely deduced from that of the sulphate, but not in the latter. If the rock were rich in sulphur it might happen that calcium sulphate would be thrown down with the fluoride, but this should be removed by thorough washing. If not, and it were certainly the only impurity present, the fluorine could be calculated, after conversion of the fluoride into sulphate, by the formula.

$\mathrm{CaSO}_{4}-\mathrm{CaF}_{2}: 2 \mathrm{~F}::$ Diff. between impure $\mathrm{CaSO}_{4}$ and $\mathrm{CaF}_{2}: x$.

It is an exceptional case when there is exact agreement between the weight of fluoride and sulphate, and with the small amounts usually met in rocks the error may be an appreciable one in percentage of fluorine, though of no great significance otherwise.

c. ACCURACY OF THE METHOD.

It is evident from the above that if the greatest care is not taken to prevent any kind of contamination of the weighed fluoride the error in the small amounts in question may be very great. If phosphate were present it would not do to apply the check by conversion to sulphate, because the evaporation with sulphuric acid and following ignition would volatilize metaphosphoric acid.

There is another source of error due to the solubility of the fluoride itself in water and acetic acid. F. P. Treadwell and A. A. Koch ${ }^{a}$ have investigated the solvent effect of these and some other reagents. They find it possible to detect additional fluorine by repeating the precipitation with calcium chloride in presence of sodium carbonate, and the total errors to be such that for 5 milligrams $\mathrm{CaF}_{2}$ or less the results are much too low, though the qualitative detection can be still made in 100 $\mathrm{cm}^{3}$ of solution with only 0.0009 gram $\mathrm{CaF}_{2}$, notwithstanding the fact that the quantitative tests had seemed to indicate that the loss would be about 0.0015 gram $\mathrm{CaF}_{2}$ for every $100 \mathrm{~cm}^{3}$, including wash water. It thus appears that with 1 gram of sample amounts of less than 0.04 to 0.05 per cent of fluorine will entirely escape observation.

A source of error not generally known arises whenever calcium fluoride is ignited in contact with paper. Fluorine escapes to a slight extent and is replaced by oxygen this reaction being doubtless due to the action of water vapor and not to oxygen of the air.

F. Seeman ${ }^{b}$ has subjected all the known methods of determining fluorine to extended examination, using calcium fluoride as his test material, and declares the Berzelius method to be far inferior to those based on volatilization of the fluorine as silicon fluoride, by reason of

a Zeitschr. anal. Chemie, vol. 43, 1904, p. $469 . \quad$ b Zeitschr. anal. Chemie, vol. 44, 1905, p. 343. 
great and varying losses, the causes of which he was unable to satisfactorily trace. He was unable to recover by it more than about 87 to 89 per cent of the fluorine. My tests do not confirm his very unfavorable results. I was able repeatedly to regain 95 to 98 per cent of the fluorine. To do so requires, however, refusion of the residue left after leaching the alkali-carbonate melt, including in the same refusion the silica precipitated by ammonium carbonate and ammoniacal zinc oxide, the treatment of the filtrate from the precipitate of calcium carbonate and calcium fluoride with additional sodium carbonate and calcium chloride, and the reevaporation of the aceticacid solutions of the precipitated calcium carbonate.

\section{DETERMINATION OF SILICA.}

The several precipitates obtained by ammonium carbonate and zinc oxide, together with the residue from the original alkali fusion, are dissolved as far as may be in hydrochloric acid, the papers ignited and their ashes added, and silica separated by the processes detailed in 6, page 68. These may be greatly simplified, if bases are not likewise to be determined, by evaporating the first filtrate from the silica with much sulphuric acid as in the last part of f. $\alpha$, page 88 .

\section{SULPHUR.}

\section{A. DETERMINATION OF ITS CONDITION.}

Before proceeding to the determination of sulphur, its condition, if present, should be ascertained.

Evolution of hydrogen sulphide on boiling with diluted hydrochloric acid is evidence of a soluble sulphide, usually pyrrhotite, but possibly lazurite. Extraction of magnetic particles reacting for sulphur shows pyrrhotite to have been in part at least the source of the hydrogen sulphide. A reaction for sulphuric acid in the filtered solution indicates a soluble sulphate, usually a silicate sulphate, noselite, or hauynite. If the residue, when well washed and treated with aqua regia or hydrochloric acid and bromine, gives more sulphuric acid, the probable presence of pyrite is shown. Should this solution likewise show arsenic, the sulphide may be arsenopyrite, which, however, is of very rare occurrence in igneous rocks, if, indeed, it is ever found there.

It is most uncommon to find any sulphur in rocks in the ordinary simple sulphate condition, except sometimes water-soluble traces, even when considerable pyrite is in evidence. The barium of fresh and even moderately decomposed rocks is apparently always in silicates and not in combination with sulphur. 


\section{B. QUANTITATIVE DETERMination.}

a. TOTAL SULPHUR.

Although the sulphur of sulphides may sometimes be correctly determined by extraction with aqua regia or some other powerful oxidizer, in the wet way, this is not aways so. Therefore it is better by far to fuse with sulphur-free sodium carbonate and a little niter over the Bunsen burner, and for a few moments over the blast, using throughout the heating the arrangement shown in fig. 3 (p. 30), in order to exclude access of sulphur from the flame gases. This determination may very conveniently be combined with those of barium, titanium, zirconium, and rare earths (p. 116). After thorough disintegration of the fusion in water, to which a drop or two of alcohol has been added for the purpose of reducing and precipitating any manganese dissolved as manganate, the solution is filtered and the residue washed with a dilute solution of sodium carbonate. In the filtrate $\left(100\right.$ to $250 \mathrm{~cm}^{3}$ in bulk) the sulphur is precipitated at boiling heat or on the steam bath by barium chloride in excess, after slightly acidifying in the cold by hydrochloric acid Evaporation to dryness first with acid, in order to eliminate silica, is needless, for in the above bulk of solution there will almost never be the least separation of silica with the barium sulphate. ${ }^{c}$ It is well that this is so, for evaporation on a bath heated by gas to remove silica would in many cases involve an error fully equal to the sulphur present by contamination from the sulphur of the gas burned. With a steam bath this difficulty does not present itself.

Owing to the small amount of sulphur in rocks, special purification of the barium sulphate obtained is hardly ever needful, especially as it has been precipitated in the absence of iron. Should there be fear of a trace of silica being present, it can be removed by a drop of hydrofluoric and sulphuric acids before weighing the barium sulphate. ${ }^{b}$

\section{b. FRACTIONAL DETERMINATIONS.}

If traces of water-soluble sulphates are present, they can be removed when desired by boiling water.

For the quantitative extraction of the sulphur of all soluble sulphates, simple boiling with hydrochloric acid suffices, which should be done in an atmosphere of carbonic acid if pyrites or other oxidizable sulphides are present, and should be finished as quickly as possible in order to minimize the error resulting from oxidation to sulphuric acid

\footnotetext{
a The results of C. W. Stoddart (Jour. Am. Chem. Soc., vol. 24, 1902, p. 852) show, in the determination of sulphur in coals by the Eschka method, often higher sulphur when silica is not removed by evapor:ltion in the usual way, but this is contrary to all my experience in silicate work, where duplicates agrce usually exactly, and if they differ are as likely to be high by one way as by the other. The explanation may possibly be that Stoddart acidified his solutions hot or used too strong an acid and had some silita precipitated.

$b$ In Jour. Soc. Chem. Ind., vol. 24, 1905, p. 10, and Chem. Zeit., vol. 29, 1905, p. 29, I. Pattinson and J. T. Dunn call attention to certain sources of error in the determination of the sulphate ion. as the presence of sulphides in some barium chloride and the use of red rubber stoppers.
} 
of the sulphur of sulphides, if present, by any ferric salts that may have been dissolved.

If soluble sulphates and sulphides as well as insoluble sulphates and sulphides are present together, the sulphur of the first is found in solution after extraction by hydrochloric acid in a carbon-dioxide atmosphere, and that of the decomposable sulphides by collecting the hydrogen sulphide evolved. ${ }^{a}$ In the residue the sulphur of the insoluble sulphides can be estimated; or from the total sulphur found in another portion its amount can be calculated. The error involved in the above estimation of the sulphur of soluble sulphides, due to the possible reducing effect of hydrogen sulphide on ferric salts, is probably negligible. Most of the hydrogen sulphide would be expelled before any such action could take place and probably before the ferric salts were largely attacked, but of course the small proportion of sulphur set free as such from pyrrhotite would escape estımation and introduce further uncertainty. In general, it would be safe enough to assume the composition $\mathrm{Fe}_{7} \mathrm{~S}_{8}$ for pyrrhotite. However carefully all these separate determinations may be carried out, the final figures for ferrous and ferric oxides can hardly be regarded as more than approximations when much sulphide is present (see p. 138, c. $\alpha$ ).

\section{BORON.}

To the best of my belief it has never been found necessary in this laboratory to determine boron quantitatively in a silicate rock. Should this determination be required it would be necessary, since most borosilicates are insoluble minerals, to fuse with sodium carbonate and apply in one of its modifications the Rosenbladt-Gooch method, ${ }^{b}$ depending on expelling the boron as methyl ester by distil-

\footnotetext{
$a$ With pyrrhotite a small fraction of its sulphur-one-eighth if the formula $\mathrm{Fe}_{7} \mathrm{~S}_{8}$ is adopted-is liberated as free sulphur and not as hydrogen sulphide.

$b$ Th. Rosenbladt (Zeitschr. anal. Chemie, vol. 26, 1887, p. 21) used magnesia for binding the boron, while F. A. Gooch (Proc. Am. Acad. Arts Sci., 1886, p. 167; Bull. U. S. Geol. Survey No. 42, 1887, p. 64; Chem. News, vol. 55, 1887 p. 7) preferred lime, as more active and reliable. Gooch and L.C. Jones have later (Am. Jour. Sci., 4th ser., vol. 7, 1899, p. 34, Chem. News, vol. 79, 1899, pp. 99, 111) upheld the use of lime and proposed as a convenient though perhaps not quite so perfect substitute, sodium tungstate containing an excess of tungstic oxide. In this article they likewise indicate the precautions now used to insure complete collection and retention of the boron.

For a useful modification in the way of collecting the boric ether in ammonia before bringing in contact with the lime, see S. L. Penfield and E. S. Sperry (Am. Jour. Sci., 3d ser., vol. 34, 1887, p. 222); also H. Moissan (Comptes rendus, vol. 116, 1893, p. 1087; Bull. Soc. chim., vol. 12, 1894, p. 955), who modifies the Gooch distilling apparatus in certain respects.

G. W. Sargent (Jour. Am. Chem. Soc., vol. 21, 1899, p. 858) obtained satisfactory results with tourmaline by collecting the boron in sodium-hydroxide solution, neutralizing the alkali with hydrochloric acid by the aid of methyl orange, then titrating the freed boric acid by sodium hydroxide in presence of glycerin, using phenolphthalein as indicator (method of Thompson, Jour. Soc. Chem. Ind., vol. 12, 1893, p. 432).

The latest contributions to the application of this method are by R. J. Manning and W. R. Lang (Jour. Soc. Chem. Ind., vol. 25,1906 , p. 396), who determine the boron in the distillate gravimetrically as the barium salt $\mathrm{Ba}\left(\mathrm{BO}_{2}\right)_{2}$, and by Wilson H. Low (Jour. Am. Chem. Soc., vol. 28, 1906, p. 807) who modifies the distillation feature, with much apparent advantage, by the addition of dry granulated calcium, chloride, and perfects the turmeric-paper qualitative test.
}

Bull. $305-07-11$ 
lation with methyl alcohol from an acid solution and collecting this in some suitable manner. For simple borates, natural or artificial, this method gives entire satisfaction when all needful precautions are carefully observed, but its application to borosilicates yet needs investigation, in view of the as yet unexplained very discordant results obtained some years ago by J. E. Whitfield in this laboratory on the mineral warwickite, a borotitanate of magnesium and iron. Perhaps his difficulty arose from the fact that a single fusion with an alkali carbonate does not remove all the boron from a borosilicate, as shown by G. W. Sargent ${ }^{a}$ and W. E. Ford, ${ }^{b}$ a cause which would doubtless be inoperative in the case of rocks, by reason of the small amounts of boron involved. For this last reason it is quite possible also that the accurate determination of but a few milligrams at most of boric oxide would not be feasible by any one of the proposed modifications of collecting and weighing or titrating it. In any case fluorine, if present, would have to be first removed by calcium nitrate or acetate before freeing the boron.

\section{CERTAIN CONSTITUENTS IN MINUTE TRACES.}

If, as sometimes may happen, the problem is presented of examining rocks for traces of gold, silver, and other elements which are not ordinarily looked for, as in F. Sandberger's investigations bearing on the origin of the metalliferous contents of veins, large weights of material must be taken, up to 50 grams or. more. This involves the use, also, of large quantities of reagents, the purity of which must then be looked to with the utmost care. Special directions to meet such cases can not now be given, nor even a complete reference list of the scanty and scattered literature on this subject. Sandberger's own writings deal but little with its analytical side, and from its inaccessibility in the Washington librarìes I am as yet unacquainted with the report by H. Von Foullon " "Ueber den Gang und die Ausführung der chemischen Untersuchung," following Sandberger's own paper ${ }^{d}$ in the general report "Untersuchungen der Nebengesteine der Pribramer Gänge." I have published a few data as to gold, silver, lead, zinc, etc., ${ }^{e}$ in S. F. Emmons's report on "The Geology and Mining Industry of Leadville;" J. S. Curtis, ${ }^{f}$ in his report on "The Silver-Lead

a Jour. Am. Chem. Soc., vol. 21, 1899, p. 858.

b Am. Jour. Sci., 4th ser., vol. 14, 1903, p. 195.

c Jahrbuch der Bergakademie, Leoben u. Pribram, 1887, p. 363.

d From Sandberger's report it appears that the rocks were treated successively with water, acetic acid, boiling dilute hydrochloric acid for two days, and finally hydrofluoric acid, the several extracts and final residue of fluorides (and pyrite) being separately examined for heavy metals. The products of distillation were also examined. A striking iact observed in all cases was the complete insolubility of the pyrite, even after the severe treatment mentioned. This speaks strongly in favor of the correctness of ferrous iron estimations in silicates by the hydrofluoric and sulphuric acid method when pyrite is present unaccompanied by other sulphides. (See $a$, p. 138.)

e Mon. U. S. Geol. Survey, vol. 12, 1886, Appendix B, pp. 592-596.

$f$ Mon. U. S. Geol. Survey, vol. 7, 1884, pp. 120-138. 
Deposits of Eureka, Nev.," has given his method of assaying rocks for traces of gold and silver; in volume 7 of the Reports of the Missouri Geological Survey, page 740, are to be found the methods used by James $\mathrm{D}$. Robertson for the determination of lead, zinc, and copper in silicate and carbonate rocks of Missouri. The earlier work of J. G. Forchhammer ${ }^{a}$ and L. Dieulafait, ${ }^{b}$ the latter of whom examined many hundreds of Archean rocks, was of a qualitative nature.

\section{THE GASES AND VAPORS EXPELLED BY HEAT.}

That rocks and minerals evolve large quantities of gases and vapors when heated is a well-recognized fact. Numerous analyses have shown that the volatile products usually comprise hydrogen and carbon dioxide as preponderating constituents, with carbon monoxide, methane, nitrogen, hydrogen sulphide, etc., in smaller amounts. The total volume may be many times that of the solid. To a certain extent, sometimes a large extent, especially as to carbon dioxide, the source of these is entrapped fluid inclusions, whose origin was contemporaneous with that of the containing minerals. A further portion may have been held dissolved in the rock, particularly in those not wholly crystallized. But from the experiments of some recent investigators, especially Morris W. Travers, ${ }^{c}$ A. Gautier, ${ }^{d}$ and K. Hüttner, ${ }^{e}$ it is very evident that the gases are often produced by chemical changes resulting from the heat used for their expulsion. Thus, hydrogen may be due to reduction of water by minerals susceptible of oxidation at high teńperatures, as ferrous silicates; the carbon monoxide to reduction of carbon dioxide in a like manner or by the hydrogen formed by the first reaction; the methane might arise from interaction between water and traces of metallic carbides, etc. $f$

Free oxygen is not reported as one of the evolved gases, and from the nature of the case it should hardly be expected.

Nitrogen, though found in but small relative amount in the free state, is comparatively abundant and easily detectable as ammonia or ammonium salts in certain classes of rocks and was long since thus recognized. H. Rose ${ }^{g}$ says that pitchstone gives off ammoniacal water on heating; A. Delesse ${ }^{h}$ found ammonia in rocks, and A. Gautier

\footnotetext{
a Pogg. Ann., vol. 95, 1855, p. 60.

$b$ Ann. chim. phys., vols., $15,17,18,21,1878-1880$.

c Proc. Roy. Soc., vol. 64, 1899, p. 130.

${ }^{d}$ Comptes rendus, vol. 131,1900 , p. 647 ; vol. 132,1901 , pp. 58 and; 189 ; vol. 136,1903 , p. 16 ; Ann. chim. phys., 7th ser., vol. 22, 1901, p. 97; Ann. des Mines, 10th ser., vol. 9, 1906, p. 316.

e Zeitschr. anorg. Chemie, vol. 43,1905 , p. 8.

$f$ For the methods of collecting and analyzing such gaseous mixtures consult Travers, M. W., The Experimental Study of Gases; the above-cited papers of Gautier; and Moureu, Charles, Comptes rendus, vol. 142, 1906, p. 44.

$g$ Quantitative Analyse, Finkener ed., p. 673.

$h$ Ann. des mines, vol. 18,1860 , p. 151.
} 
later made quantitative tests; $\mathrm{H}$. Erdmann ${ }^{a}$ found that ammonia was obtained by acting on various minerals of ancient igneous rocks with a caustic alkali; C. Luedeking and H. A. Wheeler ${ }^{b}$ found ammonium sulphate in a barite from Missouri, the presence of which I was able to confirm.

It has been noted in this laboratory on three separate occasions, when series of ores, roofing slates, and eruptive rocks were analyzed, that ammonia, either in the form of chloride or sulphate, or even as free ammonia, was given off on heating. Its appearance was not limited to one or a few specimens of a series, but seemed to be characteristic of all and to be afforded by the unbroken rock as well as by the powdered sample. The precise conditions under which the specimens were collected not being known, it is impossible to affirm positively that the ammonia may not have been due to recent organic contamination of some sort, especially in the case of the slates, but it is believed that a more critical collection of material will not alter the general result. Its amount was sometimes readily determinable by Nesslerization, being as high as 0.04 per cent in some slates. Carbonaceous organic matter was absent from most of these, but doubtless existed in them in their early history. In their case the ammonia was, in part at least, evolved as such, imparting a strong alkaline reaction to the water in the upper part of the tube. The presence of sulphides, fluorides, or chlorides in the rock might cause the ammonia to appear as a sublimate of sulphate, fluoride, or chloride. It has been suggested that ammonia might result from the action of water on metallic nitrides, $O$. Silvestri ${ }^{c}$ having observed a nitride of iron on a lava from Etna.

\section{SPECIAL OPERATIONS.}

The problem often presents itself of ascertaining the composition of that portion of a rock powder which is soluble in special reagents or in a reagent of a particular concentration. No precise directions can be formulated to meet such cases. The procedure must vary with the character of the constituents of the rock and with the object which it is sought to attain, and only in exceptional cases can a separation of this kind be sharp. Much depends on the degree of fineness of the powder and on the length of time it is exposed to the action of the reagent.

\section{A. DETECTION OF NEPHELINE IN PRESENCE OF OLIVINE.}

For confirmation of the microscopic diagnosis, Prof. L. V. Pirsson ${ }^{d}$ has indicated a means of detecting nepheline in presence of olivine, as in nepheline basalts, based on the very ready solubility of nepheline, as

a Ber. Deutsch. chem. Gesell., vol. 29, 1896, p. 1710.

$b$ Am. Jour. Sci., 3d ser., vol. 42, 1891, p. 495. c Gazz. chim. ital., vol. 5, 1875, p. 301.

d Am. Jour. Sci., 4th ser., vol. 2, 1896, p. 142. 
compared with olivine, when boiled for but one minute with a sufficiency of very dilute nitric acid $(1: 40)$. Gelatinization of the filtrate on evaporation is taken as evidence of the presence of nepheline. If olivine is present in quantity, however, this test must not be accepted at once as final, for some, if not all, olivines are much more soluble in nitric acid of the above strength than Professor Pirsson was led to believe from his original tests. If, therefore, on evaporation of the filtrate, much iron is indicated, the gelatinization may well be due to olivine alone or in part, and then the quantitative relation of silica to iron plus magnesium should be ascertained. It.must also be borne in mind that any other very soluble silicates present will be more or less affected, and that apatite is largely or wholly dissolved. It is possible that still more dilute nitric or perhaps some other acid may exert a slighter solvent action on olivine without being appreciably less effective in dissolving nepheline, etc. In combination with a quantitative analysis of the extract the method is perhaps susceptible of a wider application than the particular case for which it was first used. It is well worth further study.

\section{B. DETERMINATION OF SOLUBLE SILICA.}

Very often in treatment by acids silica is separated in gelatinous or granular form mixed with the unattacked minerals, and it becomes necessary to remove or estimate this silica, or else to discriminate between soluble and insoluble silica already existing together. Usually a boiling solution of sodium carbonate has been employed for this purpose, though the caustic alkalies have found advocates.

G. Lunge and C. Millberg ${ }^{a}$ have conclusively shown that quartz is not nearly so insoluble in solutions of the caustic alkalies as has been supposed, but that given a sufficient degree of subdivision it can be brought wholly into solution; that it is impossible to secure correct separation of quartz and opaline silica by the use of either caustic or carbonated alkalies; and that digestion on the water bath for fifteen minutes with 5 per cent solution of sodium carbonate is the only way to secure exact separation of unignited precipitated silica from quartz, and then only when the finest flour has been removed by levigation. The authors say:

\footnotetext{
If, however, no more of such flour is present than is produced in the ordinary operations of powdering and sifting through cloth of the finest mesh, the error arising from the abovementioned treatment is so slight that it can generally be neglected; it reaches 0.1 to, at the most, 0.2 per cent of the total silica, by which amount the quartz will appear too low, the amorphous silica too high.
}

The above authors also show, however, that the solvent action of the caustic alkalies on quartz becomes very apparent only when the material has been reduced to such an utterly impalpable degree of 
fineness as is practically never reached in the preparation of samples for rock analysis. For this reason $I$ have no hesitation in recommending the employment of a dilute solution of sodium hydroxide when the silica separated by acid from one of several mineral constituents of a rock is to be estimated. Even when dilution is considerable, solution is almost immediate, and as soon as this is accomplished-the point being known by the change in appearance of the residue-the solution should be diluted with cold water and filtered at once. The difficulty met with in filtration may often be overcome by faintly acidifying, which has the added advantage of at once arresting any further action of the alkali. If the dilution is sufficient no separation of silica results from so doing. Very dilute acid should also be used for washing. Lunge, when using sodium carbonate, washes with hot carbonate solution to which alcohol has been added, thus obtaining clear filtrates.

B. Sjollema ${ }^{a}$ regards diethylamine as suitable for the separation of amorphous silica from quartz and silicates in soils. He found that a 33 per cent boiling aqueous solution of the reagent dissolved 0.65 gram of amorphous silica practically wholly after eight hours action and a 16 per cent solution within 1 per cent, whereas the solubility of quartz powder was only 1.2 per cent after two days.

a Jour. landw. Chemie, vol. 50, 1902, p. 371; abstracts in Jour. Chem. Soc., vol. 84, 1903, p. 241; Analyst, vol. 28, 1903, p. 123; Chem. 7eit., Rep., 1903, p. 21. 


\title{
CARBONATE ROCK ANALYSIS.
}

\author{
PART I.-INTRODUCTION.
}

29. QUALITATIVE COMPARISON OF CARBONATE AND SILICATE ROCKS.

The analysis of an ideally pure carbonate of any one metal is of great simplicity. Occasionally the arbonates met with in nature approach but probably never fully reach this condition of purity, as when we find them in the form of well-crystallized mineral species, like calcite, magnesite, siderite, cerussite, etc. Even in such cases it is usually true that the crystals either contain included foreign matter to a slight extent or are composed of more than one carbonate in isomorphous mixture, and then the separation of the different metals may demand considerable skill and time. The great carbonate formations of the earth's crust are never of this simple character, however, even the most dazzling white marble contains other ingredients than calcium carbonate, and the degree of admixture may vary from almost nothing up to that indefinite point beyond which the rocks cease to be classified as limestones or dolomites, but rather as calcareous shales, sandstones, etc.

The matters other than carbonates which these rocks may contain are in the main original constituents, having been introduced at the time the carbonates were themselves deposited. They may consist of the detritus of earlier geologic formations in the form of sand or clay that has undergone little or no alteration in the subsequent passage of time, besides carbonaceous matter derived from the marine life existing during their formation. But besides these there may be found, secondary minerals that have resulted from chemical transformations in the mass of the accumulated sediments, either with or without the powerful aid of dynamic forces like long-continued movements of the crust or the intrusion of hot igneous rocks. The metamorphism produced by these last agencies is often of a most profound character and may result in the production of a great variety of minerals altogether foreign to the original sediments. The metamorphism may have been accompanied by the introduction and incorporation of new material, but even in the contrary case all the chemical constituents of the original detrital foreign matter have necessarily become a part of the carbonate rocks. These constituents are furthermore necessarily those common to silicate rocks of the earth's crust, hence the carbonate 
rocks differ qualitatively in no essential respect from their silicate associates except as to the more common occurrence of carbonaceous matter; and a complete analysis of one of them involves most of the determinations and separations described in the first part of this work. The analytical procedure differs, however, in some respects, owing to the great preponderance in most cases of components easily soluble in the common mineral acids, which either renders it unnecessary to use an alkaline flux or enables the analyst to reduce its amount very materially, thereby simplifying subsequent operations. The analysis is further simplified by the occurrence in reduced amounts, as a rule, of those elements which most complicate the analysis of a silicate rock. Only in the very purest carbonate rocks are some of them nearly or entirely lacking, as silicon, aluminum, and the alkalies, but the minor constituents zirconium, barium, and strontium are often not present in readily determinable amount.

In the following pages the descriptions will apply especially to those large deposits of carbonate materials denominated limestones, magnesian or dolomitic limestones, and dolomites, many of which are of great and growing economic importance. For the purpose of the analyst an exact definition of these terms is of minor significance. Indeed, among geologists there has been no uniform rule for their delimitation, and from the necessity of the case any rule must be purely arbitrary, since there is absolutely no break in the series between limestones and dolomites. The last name strictly applies only to the equiatomic double salt of calcium and magnesium, $\mathrm{CaMg}\left(\mathrm{CO}_{3}\right)_{2}$, and by some it is held that this should be its only application. On the other hand, the opposite extreme has been approached in contending that all limestones with as much as 1 or 2 per cent of magnesium carbonate should be classed as dolomites. According to H. Ries ${ }^{a}$ the most prevalent practice among geologists seems to be to call all limestones with from 5 to 18 per cent of magnesium carbonate magnesian limestones and the rest limestones or dolomites, according as their magnesium-carbonate contents lie below or above these limits. Some, however, while subscribing to the above definition of magnesian limestones, would and do call those rocks with from 20 per cent of magnesium carbonate up to the dolomitic ratio dolomitic limestones.

It has seldom been considered necessary to analyze the carbonate rocks with any great attention to detail. The majority of analyses have been made with reference to their technical utilization and with determination of only the more important constituents. In such cases it has been a common practice to omit direct determination of the carbon dioxide and either to calculate its supposed amount on

$a$ Bull. New York State Mus. No. 44, 1901, p. 644. 
the basis of the total lime and magnesia found or to regard the loss on ignition as representing the carbon dioxide. The portion insoluble in hydrochloric acid has been frequently stated as silica. Any iron that may have been determined has been reported as $\mathrm{Fe}_{2} \mathrm{O}_{3}$, water and carbonaceous matter have been entirely overlooked or neglected, as also titanium, phosphorus, and the rarer constituents, and sulphur has been almost uniformly reported as $\mathrm{SO}_{3}$. Such practice has repeatedly involved very gross errors of omission as well as of commission, which, though of little moment in many cases from the commercial point of view, detract much from the value of all these analyses in the eyes of the geologist intent on gaining an exact knowledge of an important formation and of the possible sources of metallic ore deposits of economic value, whether these last are residual soils resulting from the subaerial decomposition of limestone formations or whether they are deposits of the ores of lead, zinc, vanadium, etc., that have been formed beneath the surface by the agency of circulating waters.

\section{MINERAL COMPOSITION OF CARBONATE ROCKS.}

The chief mineral compounds entering into the make-up of the carbonate rocks are of course calcium carbonate for the limestones proper, with the double carbonate, the mineral dolomite, in increasing proportions through the magnesian limestones and dolomites; but it is the exception to find them free from other carbonates as essential components, chiefly those of iron and manganese, which are usually in isomorphous combination with the constituents of one or the other of the above minerals. The proportion of these is far less than that of the others, but sometimes aggregates several per cent. When they predominate the rock is no longer a limestone, but becomes an ore of iron or manganese.

Neglect to take account of these minor carbonate constituents is a frequent source of error in the statement of analyses, where it is very common to find reported as carbonates only calcium and magnesium, and perhaps a considerable amount of iron as $\mathrm{Fe}_{2} \mathrm{O}_{3}$ and manganese as one of its higher oxides, although it is practically certain that these must have existed in large part as ferrous and manganous carbonate. A careful determination of the carbon dioxide will often tell whether this is so or not, for it will perhaps be found in excess of that required for lime and magnesia. A negative result, however, is not to be taken as proof of the absence of iron or manganese carbonate or both, for very often a small portion of the magnesia may be in silicate combination, or in some formations there may be a little gypsum to claim a portion of the lime. This last is not likely to be the case in the average hard rock that is used for the manufacture of cement. In such the sulphur often reported as $\mathrm{SO}_{3}$ is likely to come from iron pyrites and perhaps to a limited extent at times from carbonaceous matter. 
It is hence easy to see that except in absence of iron and manganese it is not possible to ascertain the exact proportions of calcium and magnesium carbonates, and even then sometimes only after separate analyses of the parts soluble and insoluble in dilute acid have been made.

The commonest sulphide in limestone formations is pyrite, though its presence is often obscured by its extremely fine state of division or by carbonaceous matter. In the metamorphosed limestones, especially those altered by intruded igneous bodies, other sulphides, as sphalerite and galena, are not uncommon, and these and still other sulphides may form important ore bodies at times or again give rise by oxidation to equally or even more valuable ores of a different sort. The strongly metamorphosed siliceous limestones are, however, seldom subjected to chemical analysis, especially for commercial purposes.

The siliceous components may be of the most varied character. Their names alone might fill a page. Suffice it to mention for the metamorphosed rocks garnet, vesuvianite, wollastonite, tremolite, diopside, the scapolites, tourmaline, apatite, and silica in the form of chert. Cherty limestones are extremely common and their silica was doubtless introduced during metamorphism. The chief siliceous components of unmetamorphosed limestone rocks are, as said before, sand and a clay. The latter particularly, since it is a clay, carries water, and this fact is usually overlooked in ordinary analyses. The analytical statements may show no water, though clayey matter is present to a large extent. The sand may be largely or altogether quartz, or it may be composed in part of such other minerals as are commonly found in sands, like mica, garnet, zircon, etc. These latter may be often detected, even in the less siliceous and clayey limestones, by dissolving large amounts in hydrochloric or acetic acid and subjecting the residue to microscopic examination, after burning off obscuring carbonaceous matter, if necessary. The unignited residue will, needless to say, contain the pyrite of the rock and any other sulphides not attacked by dilute acid. In the case of some nonsiliceous rocks it may even consist to a considerable extent of iron oxide, probably in most cases one of the hydrated oxides, but perhaps often hematite. The presence of such a constituent in a limestone is occasionally indicated by a reddish cast of color in the fresh rock, provided there is not enough carbonaceous matter to obscure it. The existence of a ferric oxide is not always incompatible with the simultaneous presence of this carbonaceous matter.

A purple color may be due, as in some slates, to admixture of a ferric oxide or hydroxide with carbonaceous matter, while to the latter component are due the gray and black colors often encountered. The color of greenish sedimentary limestones may be caused by certain minerals of the mica family, as the chlorites. 
The condition of phosphorus when it occurs in only minute amounts is not determinable with certainty. It may sometimes doubtless be found in traces in the form of heavy phosphates like monazite and xenotime in the insoluble residues, and at others it is held by iron and aluminum, but in the highly phosphatic rocks its usual form is overwhelmingly that of phosphorite or apatite, which may, in fact, become the predominating constituent.

It is known from my analyses that vanadium is a regular though very minor constituent of limestones as well as of sandstones and igneous rocks generally. Recent tests on 100-gram lots of a great limestone formation in northwestern Mexico show that it at least carries molybdenum as well as vanadium, and also nickel. Nothing is known with certainty regarding the mode of combination of these elements in limestones, though it is quite possible, as to the vanadium at least, that it was an original ingredient of the aluminous silicates of the clayey matter, and that nickel, zinc, and lead when present are mainly in the form of carbonate or sulphide.

Carbonaceous matter has been frequently alluded to as a common constituent of secondary importance. It is absent from the white marbles and some colored decorative stones, but forms an appreciable percentage of other limestones in extreme cases.

Some kinds of bituminous limestone are characterized by a fetid odor when struck or abraded. This is perhaps due in most cases to hydrogen sulphide, either original or resulting from decomposition of organic matter. The presence of hydrogen sulphide is sometimes unmistakable, and Prof. B. J. Harrington has determined its amount at about 0.02 per cent in the form of liquid inclusions in a fetid calcite from Canada.

The above remarks do not exhaust the subject of the mineral composition of limestone rocks, this term being used here and elsewhere in its generic sense to include the magnesian varieties. They may serve as a sufficient guide, however, to those undertaking their analysis. Ignorance of the mineral composition in respect to the minor components has been a prolific source of error on the part of those analyzing these rocks.

31. BEHAVIOR TOWARD DIFFERENT REAGENTS AS A MEANS OF DISTINGUISHING DIFFERENT CARBONATES.

Although for the analyst provided with laboratory facilities it is usually of little importance to possess other means for identifying qualitatively the different varieties of carbonate rocks than the conventional methods of qualitative analysis, some of the rapid methods for so doing may serve a most useful purpose at times, especially in the field, where transport of bulky apparatus and quantities of liquid reagents is difficult or impossible. 
a. DIFFERING SOLUBILITY IN TARTARIC AND CITRIC ACIDS AND IN ACID POTASSIUM SULPHATE.

This subject, touched on now and then by earlier writers, was first studied by H. C. Bolton, ${ }^{a}$ and many of his results were long afterwards confirmed by J. W. Richards and N. S. Powell. ${ }^{b}$ Solid organic, acids like citric and tartaric were found to be well adapted for distinguishing between many carbonate minerals by the difference in the degree of action on them. Potassium acid sulphate had earlier been used to some extent by E. Jannettaz, ${ }^{c}$ and Richards and Powell give data as to this reagent for carbonates. It will suffice here to refer to the originals, especially Bolton's papers, where will be found data as to the solubility of a great variety of minerals in the abovenamed solvents as well as in others of inorganic nature.

For the present purpose it is more important to present in brief the methods proposed for distinguishing between calcite and aragonite and between relatively pure limestones and those high in magnesium.

b. THE MEIGEN REACTION FOR DISTINGUISHING CALCITE FROM ARAGONITE.

According to $W$. Meigen, ${ }^{\not}$ calcite powder when boiled with a dilute solution of cobalt nitrate remains white or, in presence of organic matter, becomes yellowish, while aragonite turns lilac red from formation of a basic cobalt carbonate. G. Panebianco ${ }^{e}$ obtained different results. Calcite after a minute's boiling with the cobalt nitrate reagent became sky blue and after four minutes lavender blue. Aragonite became at once lilac and later violet, the difference in the colors being supposedly due to different basic carbonates. Panebianco also observed that the reaction was useless for proving absence of calcite when mixed with aragonite, for the color characteristic of the latter still appeared when 19 parts of calcite were mixed with but 1 of aragonite. Freshly precipitated gelatinous calcium carbonate, as well as that from warm solutions, gives the aragonite reaction, but that formed at $0^{\circ}$ affords the calcite reaction.

A. Hutchinson ${ }^{f}$ seems to have shown that the disagreement between the results of these investigators is to be attributed to the presence of iron in the cobalt nitrate used by Meigen.

According to Meigen barium and strontium carbonates, but not that of magnesium, behave like aragonite, and calcium phosphate produces a blue powder.

$a$ Ann. New York Acad. Sci., vol. 1, 1877-1880, pp. 1, 153; vol. 2, p. 1; Proc. Am. Assoc. Adv. Sci., vol. 31, 1883, p. 271; Chem. News, vols. 36, 37, 38, 43, 47, 1877-1883; Rept. Brit. Assoc. Adv. Sci., 1880, p. 506; Min. Mag., vol. 4, 1880-81, p. 181; Ber. Deutsch. chem. Gesell., vol. 13, 1880, p. 726.

b Jour. Am. Chem. Soc., vol. 22, 1900, p. 117.

c Comptes rendus, vol. 77,1873 , p. 838 ; vol. 78,1874 , p. 852 .

d Centralbl. Min., 1901, p. 577.

$\epsilon$ Rivista min. crist. ital., vol. 28,1902, p. 5.

$f$ Min. Mag., vol. 13, 1903, p. xxviii. 
c. distinguishing Between CALCITE AND DOLOMite.

It must be understood that magnesian and dolomitic limestones represent, as a rule, mixtures of the double carbonate dolomite with calcite, and not of calcite with magnesite. Calcite is easily soluble in dilute hydrochloric and acetic acids; dolomite and magnesite are nearly insoluble. Hence calcite effervesces strongly with these acids, even in lump form, while dolomite shows little or no effervescence. An easy means is thus afforded for distinguishing the two. Dolomitic limestones are also but feebly attacked, according to their content in calcite, so that in powder form it is not difficult to make a fair separation of calcite from dolomite and magnesite, but not from magnesian hydrocarbonates, which last, however, are never essential components of the great carbonate formations.

J. Lemberg ${ }^{a}$ found that calcite responds by the formation of a violet color in its powder when treated with a solution of aluminum chloride and hematoxylin (extract of logwood), but that dolomite remains unaffected. The solution is prepared by dissolving 4 parts of dry aluminum chloride in 60 parts of water, adding 6 parts of logwood, and boiling for twenty-five minutes with replacement of the escaping water. 'The deep-violet solution is then filtered.

Coarsely powdered Iceland spar and Carrara marble became violet after five to ten minutes immersion and subsequent removal of the adhering solution with water. In the powder of a dolomitic limestone the calcite grains were easily distinguishable from those of dolomite by this means. It is not advisable to prolong the treatment unnecessarily, since after twenty minutes the dolomite surfaces may show occasional pale-blue spots. The reaction is caused by precipitation upon the calcite of aluminum hydroxide, which forms, with the coloring matter, a lake.

F. Hinden ${ }^{b}$ finds that limestone (calcite) at room temperature gives carbon dioxide with 10 per cent solution of ferric chloride, but dolomite does so only on warming. A second reagent mentioned by him is copper-sulphate solution, from which basic copper carbonate is separated by calcite, but is not by dolomite.

The behavior toward heat alone is sometimes useful as a distinguishing test, for dolomite loses its carbon dioxide so easily that the powder is violently projected from the crucible if the heat is not very cautiously applied, whereas calcite can be strongly heated at the start without danger of loss of solid matter. 


\section{PART II.-REFINED METHODS OF ANALYSIS.}

32. SILICA, ITS SEPARATION FROM ALUMINA, ETC.

\section{A. METHODS OF DECOMPOSING THE ROCK.}

Few limestones are so pure that they leave no residue on treatment with hydrochloric acid. The residue may consist of quartz, clay, or other silicates, carbonaceous matter, pyrite, etc., and the mode of attack employed will depend on the amount of insoluble matter, and whether or not it is desired to know its composition separately from that of the soluble portion.

a. When the inORganic residue is to be separately aNALyzed.

A gram of the rock powder is moistened with water and dissolved in dilute hydrochloric acid (or acetic, if the former acid is likely to act appreciably on the silicates) in a covered beaker till all effervescence ceases. Moderate heat is needed if the effervescence is so weak as to indicate a rock of dolomitic character. The solution is filtered through a $7 \mathrm{~cm}$. filter and the residue washed with water, or hot dilute hydrochloric acid should gypsum be present and the silicates resistant. The paper, with its contents, is ignited moist in platinum (blast needed only if the amount is considerable), and after weighing it is fused with sodium carbonate and analyzed like a silicate rock (d,p.71 et seq). If it contains hydrous minerals the original water content of the residue is best determined by a separate test on a fresh portion of the sample, and not by drying and weighing on a counterpoised filter and then igniting. The weight of water found should be added to that of the ignited residue in order to get the true weight of the insoluble matter.

If the ignited residue is wholly quartz, or is very insignificant in amount, it may be treated at once with a drop of sulphuric acid and a few drops of hydrofluoric acid, and the acids removed in the radiator shown in fig. 2 (p. 29). If then a slight residue still is visible, it is well to repeat the treatment with acids and evaporation, for it can not be too insistently pointed out that quartz resists the action of hydrofluoric acid far more than many silicates, and several evaporations may be needed to volatilize the crystallized mineral unless it has first been reduced to a most impalpable state of division. When the weight after exposure for a few minutes to the full burner heat no longer changes, the loss represents silica. If a slight residue still shows (it is most likely to be mainly alumina), the subsequently obtained precipitate of alumina, etc., is added to it and ignited in the same crucible. The filtrate is best treated as in 33. A. a, page 178 .

If the original residue is small, and accurate knowledge of its composition is desired, several grams of the rock may be dissolved and the 
whole filtrate best treated for dissolved iron, aluminum, and manganese, as in 33. A. a, page 178, but only an aliquot part of the subsequent filtrate should then be used for the determination of calcium and magnesium.

In case the filtrate from the residue is perceptibly colored by dissolved organic matter, a condition that may occasionally arise, this organic matter must be effectually destroyed before proceeding to the precipitation of iron and aluminum, for otherwise incomplete precipitation of one or both will be the result. The complete removal of this disturbing material can not always be effected by ordinary oxidizing agents, but only by evaporation and heating to its carbonization point. In doing this it is necessary, in order to avoid possible loss of iron as chloride, to evaporate with nitric acid to dryness a couple of -times and then to heat over a free flame gently till the desired result is achieved. If this seems undesirable for any reason, it may be better to start with a fresh portion of the limestone and to treat it by one of the methods under $b$, below, for the bulk analysis.

b. WHEN THE INORGANIC RESIDUE IS NOT TO BE SEPARATELY ANALYZED.

This will be the usual case. As said above, the residue may consist of clay or other silicates, carbonaceous matter, pyrite, etc., with or without quartz. There are two ways open to render the noncombustible part of this soluble.

$\alpha$. By solution in acid after strong ignition.- - This is the best method to employ in all cases where the ratio of insoluble to soluble compounds is not less than is found in an argillaceous limestone that is directly suited for burning to Portland cement-that is to say, when the silica does not much exceed 15 per cent and the oxides of iron, aluminum, and titanium together are not in excess of 6 per cent. The exact allowable limits have not yet been determined, nor is it known what the proportions may be in dolomites and dolomitic limestones. This is a subject for further investigation.

Limestones, however, in which the above percentages of silica, alumina, etc., are not exceeded may be converted in ten to fifteen minutes by a good blast capable of giving a temperature of $1,100^{\circ}$ to $1,200^{\circ}$ to a product that is wholly soluble in hydrochloric acid, provided the rock was first reduced to a very fine powder.

A gram of the powder is heated in a covered platinum crucible by an inclined blast. If a limestone, the flame may be applied at once, as a rule, without fear of loss, or after short exposure to a full Bunsen flame. Highly magnesian limestones, if this method is applicable to them, must, however, be heated with the greatest caution, for their temperature of decomposition is far below that of limestones, and violent projection of material often begins far short of visible redness. 
The strong heating is usually stopped after ten or fifteen minutes, when the shrunken product may have the appearance of a sintered or even clinkered mass that detaches for the most part readily from the crucible. $^{a}$ It is transferred to a beaker or evaporating dish and moistened with water. The crucible is then cleaned with hydrochloric acid $(1: 1)$ and the contents are poured into the beaker or dish. By gentle heat and cautious pressure with the flattened tip of a rod the lump or lumps are caused to disintegrate and pass largely into solution in a few minutes. A certain amount of silica may remain undissolved in a flocculent state, but this is of no moment. When all grit has disappeared, the liquid, if in a beaker, is washed into a dish and evaporated to dryness. If solution was made directly in the dish the volume of liquid need not exceed a few cubic centimeters and the evaporation takes but a short time. A much smaller dish is allowable than in the case of wholly siliceous minerals, because of the small bulk of solution and the entire absence of added fixed salts.

$\beta$. By solution in acid after heating with sodium carbonate.-When the siliceous components are in such amount as not to permit the formation of a wholly soluble product by strong ignition, it is necessary to mix sodium carbonate with the powder and decompose the silicates by its aid over the blast lamp. This procedure may be adopted with all siliceous limestones if desired. The amount of flux to be used is but a fraction of that needed for a silicate analysis. One-fourth to one-half gram for 1 gram of limestone suffices, ${ }^{b}$ for the lime formed by ignition is itself a powerful flux and it is quite unnecessary to have here a liquid fusion. A sintering suffices. This proportion holds also for highly magnesian limestones and for cement rocks containing as little as 50 per cent of lime. ${ }^{c}$

\section{B. SEPARATION OF SILICA.}

In this connection the remarks under $\beta$, page 76 , should be carefully read.

Because of the absence of large amounts of alkali salt and of much silica the evaporation of the hydrochloric-acid solution obtained in A. a. and b. $\alpha$, and $\beta$, takes little time as compared with a silicate analysis. When dry, or nearly so, on the steam bath, the dish may be placed in an air bath or (covered) on a platinum triangle resting on a hot plate, and heated to $200^{\circ}$ for an hour if a limestone is under treatment, but for a highly magnesian limestone

$a$ The changes that take place during the ignition comprise loss of all carbon dioxide, water. and carbonaceous matter; oxidation of all pyrite, with retention of the whole of the sulphur as calcium sulphate. Prolonged heating will gradually expel all the sulphur trioxide from the calcium sulphate and later the alkalies, which can be wholly volatilized in an hour or less by a powerful blast. With an inclined blast the alkalies condense in part on the under side of the lid as a soluble and powerfully alkaline deposit, sometimes weighing several milligrams, but in the time above set no loss of alkali appears to occur.

$b$ Shimer, P. W., in Meade, R. K., The Chemist's Pocket Manual, p. 162.

c Personal communication from R. K. Meade. 
the temperature should not exceed $120^{\circ}$, according to J. P. Gilbert, ${ }^{a}$ because of recombination of silica and magnesia, with subsequent re-solution of the silica when acid is added.

Bertram Blount claims ${ }^{b}$ that by heating to $200^{\circ}$ in this manner the silica is completely separated by a single treatment, but I have not found it so, though the amounts going into solution on subsequent addition of acid are small, seldom over 2 or 3 milligrams. Therefore, for exact work with silica present to the amount of 2 to 4 per cent and over, that already rendered insoluble should be filtered off and the solution again evaporated. With this end in view the dry mass is drenched with strong hydrochloric acid and allowed to stand for a few minutes, then as much water is added and the dish covered and placed on the bath for ten minutes. Instead of strong acid that of half strength may be used and the heating begun at once. The silica is then separated by filtration on a filter of suitable size, washed thoroughly with dilute acid and then twice with cold water. The filtrate is evaporated again to dryness, the residue extracted with hydrochloric acid as before, but with allowance of only a few minutes' time, and the solution filtered once more through a second and smaller paper. The two papers with their contents are slowly dried, charred, and ignited in platinum, finally over the blast for ten minutes. The weighed silica is to be corrected for foreign matter by evaporating with $5 \mathrm{~cm} .{ }^{3}$ of hydrofluoric acid and one or two drops of sulphuric acid, as directed in c (p. 79). The residue obtained after ignition is almost always considerably less than that found in analyzing a silicate rock and an ignition of a minute or two over a full burner, after expulsion of the sulphuric acid, is in almost all cases sufficient. The composition of this residue is similar qualitatively to that derived from silicate rocks. It should never contain calcium or magnesium, but consists mainly of alumina, with a little ferric, titanic, and phosphoric oxides. It is preserved and the subsequently obtained precipitate of these oxides is added to it and ignited in the same crucible.

33. ALUMINUM, TOTAL IRON, TITANIUM (SILICA, MANGANESE); PRECIPITATION IN COMPANY WITH PHOSPHORUS, ETC. $c$

The next steps differ widely according to the relative amounts of iron, aluminum, etc., taken collectively, and according as it is the intention to precipitate manganese with these or after separation from them. The individual preferences of experienced analysts will often differ as to the selection of the particular method, and it is not intended here to prescribe definitely the course that anyone should

$a$ Technology Quart., vol. 3, 1890, p. 61; abstract in Zeitschr. anal. Chemle, vol. 29, 1890, p. 688.

$b$ Jour. Soc. Chem. Ind., vol. 21, 1902, p. 1217.

$c$ See also 8, p. 81.

Bull. $305-07-12$ 
follow. Nor in the following are all methods of separation that may be capable of affording good results noticed. There are some that seem promising but that have not yet been studied sufficiently in this laboratory, nor in fact elsewhere, to justify description. A method that may be well adapted to a certain combination of elements may be valueless if to that combination a single other element is added; or it may be that the possible disturbing influenee of that element has not been as yet ascertained.

\section{A. PRecipitation of aluminum, iron, etc.}

a. COllective pRecipitation of aluminum, iron, manganese, titanium, and phosPHORUS BY AMMONIUM SULPHIDE.

If these elements collectively represent 2 or 3 per cent or less of the rock, the filtrate from the silica (32. B, p. 176), reduced in bulk if need be, is put into a flask of $150 \mathrm{~cm}^{3}$ capacity with enough hydrochloric acid to prevent precipitation of magnesium when made ammoniacal. This last is a most important point. Ammonia free from carbonate is then added to strong alkalinity and hydrogen sulphide is introduced to saturation, followed by a fresh addition of ammonia equal to the excess first used. Boiled water is then poured in till well up in the neck, the cork is inserted, and the flask is set aside for twelve to twenty-four hours. Its contents are then collected on a filter of small size $(7 \mathrm{~cm}$.) and washed with water containing a little ammonium. sulphide and nitrate. Precipitation is not absolute as to one or more of the constituents of the separated mixture, but the amounts recoverable from the filtrate are usually very minute. (For treatment of the filtrate see 36, p. 183.)

The paper and its contents, having been washed with ammonium nitrate, may be safely charred and ignited as they are in the crucible containing the residue left after volatilizing the silica. The final weight after ignition in full contact with air, followed by application of the inclined blast for not over five minutes, represents $\mathrm{Al}_{2} \mathrm{O}_{3}$, $\mathrm{Fe}_{2} \mathrm{O}_{3}, \mathrm{Mn}_{3} \mathrm{O}_{4}, \mathrm{TiO}_{2}$, and $\mathrm{P}_{2} \mathrm{O}_{5}$. (For the separation of these, see C, p. 180 .)

This method is inapplicable for phosphatic limestones containing insufficient aluminum to more than combine with the phosphorus, for then calcium phosphate would be precipitated also. In such case method b or c would apply if the iron and aluminum together more than sufficed to carry down the phosphorus, but in general it would be necessary to add a known amount of pure iron oxidized to the ferric state, then to precipitate by ammonia or the basic-acetate process, and to deduct the added iron oxide from the weight obtained. 
b. PRECIPITATION OF ALUMINUM, IRON, TITANIUM, AND PHOSPHORUS BY AMMONIA.

The filtrate from silica is boiled in a beaker of good glass, with addition of a few drops of bromine water or two or three of strong nitric acid, till all trace of bromine or chlorine is gone. Then hydrochloric acid is added, if not already present, in amount sufficient to prevent precipitation of magnesium when rendered ammoniacal. Ammonia is added to the boiling solution (in platinum if possible), 100 to $200 \mathrm{~cm}^{3}$ in volume, in slight excess, and the boiling is continued for two or three minutes. The precipitate is allowed to settle in the covered vessel, collected quickly on a filter, washed with hot water two or three times, and sucked dry. It is then redissolved in hot and moderately strong hydrochloric acid, the solution is boiled to drive out the trace of chlorine derived from solution of any precipitated manganese dioxide, and the precipitation is repeated just as described once, twice, or even three times, according to the amount of manganese in the rock. Macerated filter paper is added before the last precipitation if the oxides are in large amount. The final precipitate is washed with water containing a little ammonium nitrate. (For treatment of the filtrate see B, below.)

The precipitate is ignited moist in the crucible containing the residue from volatilization of the silica by hydrofluoric acid, and the combined weights increased by traces subsequently recovered ( $\mathrm{B}$, below) represent all iron as $\mathrm{Fe}_{2} \mathrm{O}_{3}, \mathrm{Al}_{2} \mathrm{O}_{3}, \mathrm{TiO}_{2}$, and $\mathrm{P}_{2} \mathrm{O}_{5}$. (For separation of the ignited oxides see C, p. 180.)

c. PRECipitation OF ALUMTNUM, IRON, TITANIUM, AND PHOSPHÓRUS BY THE Basic-ACETATE METHOD.

This method of separation is less employed in limestone analysis than in that of silicate rocks, by reason of the relatively smaller amounts of those elements that precipitate in the basic-acetate process, and hence of their more ready separation by ammonia from appreciable quantities of manganese. (For its application, see A. b. $\beta$, p. 83 , giving due regard to the smaller amounts of iron, etc., involved in the case of limestones.)

\section{B. TREATMENT OF THE FILTRATES FROM ALUMINA, ETC.}

The combined filtrates obtained in A. b (or c), above, which, besides all the calcium and magnesium, will contain the manganese and usually traces of aluminum and even iron, are evaporated to a small bulk in platinum with the addition of a few drops of ammonia from time to time, and any precipitate that separates is collected. This is washed a little, redissolved in hydrochloric acid (with addition 
of a drop ${ }^{\circ}$ or two of sulphurous acid if its color indicates precipitation of some manganese), and reprecipitated by ammonia at boiling heat in a very small beaker. If need be the precipitation is to be again repeated, but this will seldom be the case. A first precipitation is, however, called for in order to separate a little calcium carbonate that may have precipitated during the evaporation of the original ammoniacal filtrate. The alumina and perhaps trace of iron oxide thus obtained are added to that already found (A. b, c, p. 179). (For treatment of the filtrate, see 34, p. 181.)

\section{SOLUTION AND SEPARATION OF THE OXIDES OBTAINED IN A AND B.}

The same course is followed, whether method a, b, or c under A, pages 178-179, has been employed for precipitating aluminum, iron, etc. According to the weight of the ignited oxides, these are fused with from one to several grams of potassium or preferably sodium pyrosulphate ${ }^{a}$ in the covered crucible over a low flame. By using pyrosulphate instead of acid sulphate the time of fusion is greatly shortened, especially when the oxides are in a finely divided state, as after precipitation in presence of macerated filter paper.

When decomposition is complete the cooled melt is dissolved in thot dilute sulphuric acid, the solution is transferred to a capacious platinum dish and evaporated as far as practicable on the bath. Strong sulphuric acid is cautiously added, and evaporation is continued over a radiator, or on a sand bath, or by aid of a free flame applied with care to prevent spattering, till fumes of acid appear freely. When cool the melt should be rather pasty and not hard and dry. It is dissolved by the aid of heat in a moderate amount of water and the solution is allowed to digest for ten or fifteen minutes on the bath. A few flocks of silica will then generally be apparent at the bottom of the dish. Whether visible or not, the solution is passed through a 7-cm. filter, which is then washed with hot water till free from salts.

$$
\text { a. STICA (BARIUM). }
$$

The paper with the silica is ignited, weighed, and then treated with a drop of sulphuric acid and a few drops of hydrofluoric acid and the crucible is again weighed. The loss represents silica that escaped earlier separation, and its amount is to be added to that already found.

If a final residue is reddish, it is to be brought into solution by a little pyrosulphate and its solution, if clear, added to the main filtrate containing the mass of the iron. If not clear, the turbidity may be due to a trace of barium sulphate, which should of course be separated and deducted from the weight of the alumina plus other oxides. 
b. IRON.

In this solution the iron is to be determined by potassium permanganate after reduction by hydrogen sulphide, exactly as detailed under 8. B. a, page 90 . The amount of vanadium present is, so far as known, always so small as to make it unnecessary to follow the procedure under 8 . B. b, page 92 .

\section{c. TITANIUM.}

After titration of the iron the solution is treated exactly as prescribed under 8. B. a. $\gamma$, page 92 , and the titanium is determined colorimetrically (p. 110).

\section{d. ALUMINUM.}

The aluminum is determined by difference after subtracting the weights of iron and titanium oxides found as above, and also the phosphoric oxide found in a separate portion of the sample (p. 184), and further the manganoso-manganic oxide, if this had intentionally been precipitated along with the iron and aluminum as in A. a, page 178 . (For the discussion of the relative merits of this indirect and the direct methods for arriving at the aluminum, see 8. A. a, p. 81, and C, p. 93.)

\section{MANGANESE.}

\section{A. IN THE FILTRATES FROM 33. B (p. 179).}

The filtrates containing the calcium, magnesium, and manganese are caught in a flask of about $150 \mathrm{~cm} .^{3}$ capacity, $2 \mathrm{~cm} .^{3}$ of ammonia are added, and hydrogen-sulphide gas is introduced to saturation, then additional ammonia is added to equal that just prescribed. The flask is set aside, stoppered for twelve to twenty-four hours, the precipitate (perhaps colored dark by a little platinum, iron, or other heavy-metal sulphide ${ }^{a}$ ) is collected on a 7 -cm. filter and washed with a dilute solution of ammonium chloride carrying a little ammonium sulphide. $^{b} \quad$ (For treatiment of the filtrate, see 35, p. 182.)

If the manganese is not to be determined in a separate portion of the rock, the flask is then cleaned from possibly adhering sulphide with a solution of half-strength hydrochloric acid diluted with five times its volume of hydrogen-sulphide water, which is then passed through the filter in order to dissolve the manganese sulphide. The fitlrate is treated exactly as under 9 . B. b, page 98 , if the gravimetric method of determination is chosen. In view of the relatively enor-

\footnotetext{
a More than a negligible trace of iron should not appear at this point. If, however, the organic matter . of the limestone is at all soluble in acids and the method of direct solution has been employed, it may very well happen that some iron (aluminum likewise) will fail of precipitation by ammonia. The former will then appear at this stage. Hence, prior ignition of the limestone is imperative in such cases.

${ }^{b}$ This is best prepared by considerably overneutralizing a little hydrochloric acid in a small beaker, and then adding hydrogen-sulphide water or introducing hydrogen-sulphide gas for a few moments, and further diluting if need be.
} 
mous errors affecting this method when small amounts are in question (see 9. A, p. 96), it is far safer to evaporate the hydrochloric-acid solution with nitric or sulphuric acid, till no trace of chlorine is left, and to apply the colorimetric method (9. C. b, p. 99).

If the manganese is to be determined in a separate portion, the sulphide precipitate is discarded; indeed, if it is known that but a few hundredths of a per cent are present, its separation at this stage may be altogether omitted.

\section{B. SEPARATE DETERMINATION OF MANGANESE.}

Small amounts are always best determined by colorimetry, and then with greatest accuracy in a separate portion of the sample. This, if wholly soluble in acid, is to be dissolved by dilute nitric acid free from hydrochloric acid, the solution filtered if necessary, and further treated, in whole or in an aliquot part, as in 9. C. b, page 99. If not altogether soluble in acid or if the solution is colored by organic matter, it is best to heat the powder with half its weight of sodium carbonate (itself free from manganese) over a strong blast, to digest the cooled mass with nitric acid till any residue that may remain is entirely colorless, to filter into a suitable-sized flask, and to determine as in 9. C.b, page 99 .

35. COPPER, NICKEL, COBALT, ZINC, RARE EARTHS, CHROMIUM, VANADIUM, MOLYBDENUM.

It is useless, as a rule, to look for any one of these constituents in the amount of sample taken for the general analysis. From 50 to 500 grams of material will be required. This is dissolved in hydrochloric acid and the residue separated by filtration. If it is desired to examine this residue it is best done by analyzing it separately, after fusion with sodium carbonate and leaching with water. Vanadium and molybdenum will then be found in the filtrate, the other metals in the residue. The former can be determined according to 18, page 126; the latter by the usual methods.

For the hydrochloric-acid solution, which will contain the above elements mainly or wholly, the treatment given under 33. A. a, page 178, is probably best adapted and the following outline may serve as a guide.

The precipitate produced by ammonium sulphide is to be dissolved, the copper and perhaps some molybdenum precipitated by hydrogen sulphide, the filtrate oxidized and evaporated to dryness and the residue boiled with a solution of oxalic acid. The rare earths, perhaps contaminated by silica, will be thrown out and are to be washed with dilute oxalic-acid solution, ignited, treated with sulphuric and hydrofluoric acids, redissolved and reprecipitated in neutral or nearly 
neutral solution by oxalic acid, ignited, and weighed. They ean then be tested as to their character by methods to be found in special treatises on the rare earths. The filtrates are to be freed from oxalic acid by evaporation and gentle ignition and the residue redissolved and tested for nickel, cobalt, zinc, chromium, vanadium, and molybdenum by approved methods. It must not be forgotten that the filtrate from the original precipitation by ammonium sulphide may hold vanadium, molybdenum, and perhaps nickel, which are to be recovered by acidifying with dilute acetic acid.

Vanadium may usually be found and determined with sufficient accuracy in 5 grams of the limestone by fusing with sodium carbonate and further treating as in 18 . C, page 127.

\section{CALCIUM, STRONTIUM, BARIUM, MAGNESIUM (MANGANESE).}

The filtrate obtained in 33. A. a, page 178, or 34. A, page 181 , is treated for the most part as in silicate analysis (pp. 101-110).

With dolomitic limestones and dolomites it will be usually impossible to obtain a correction by the method given in C.b, page 109, for the small amount of calcium with the magnesium pyrophosphate. Possibly a better result might be had by adding to the nearly neutral solution of the pyrophosphate a few drops of sulphuric acid and a volume of alcohol equal to that of the solution, in order to precipitate calcium sulphate, but this has not been put to the test in presence of phosphoric acid, though it works well in its absence.

The accurate determination of barium in a limestone is more difficult than in a silicate rock. It is perhaps best to recover it, if present, from the filtrates from the calcium oxalate, after evaporating them and expelling ammonium salts, by taking up the residue in as little hydrochloric acid as possible and, without filtering, adding a few drops of sulphuric acid and allowing to stand for several hours. The washed and ignited precipitate is evaporated with a drop of sulphuric acid and a few drops of hydrofluoric acid to remove silica, the final residue is dissolved by a little hot strong sulphuric acid, and the resulting solution poured into a few cubic centimeters of cold water, the crucible being well rinsed with cold water by the aid of a feather or "policeman." If a precipitate now appears it is barium sulphate, which is collected and weighed in the customary manner.

For the utmost refinement in the determination of the magnesium it is probably best to evaporate the filtrates from the calcium oxalate to dryness, expel the ammonium salts by ignition, add hydrochloric acid and reevaporate, take up again with as little acid as possible, and filter through the smallest filter. The filter is preserved for later use. To the filtrate, which should be very small in volume, a few drops of sulphuric acid are added, and if after somehours a precipitate of barium 
sulphate has appeared it is collected and burned in platinum with the previous filter and its contents. The ignited matter is treated as in the preceding paragraph.

To the filtrate is added enough hydrochloric acid to prevent precipitation of magnesium when rendered ammoniacal. Ammonia in slight excess is added and the solution is boiled for some minutes in a platinum dish. There will probably appear a very slight precipitate containing aluminum hydroxide, which is to be redissolved and reprecipitated. In the filtrate the magnesium is precipitated in the manner elsewhere detailed (p. 107).

\section{PHOSPHORUS.}

The rock is dissolved in dilute nitric acid, the solution filtered, the residue fused with a little sodium carbonate, and its nitric-acid solution added to the other.

Or, if the rock is rather argillaceous, the powder may be mixed with half its weight of sodium carbonate and strongly blasted. The nitricacid solution of the mass is then evaporated to dryness to remove most of the silica, which is collected on a filter, washed, ignited, and evaporated with hydrofluoric and nitric acids. The evaporation with nitric acid alone is repeated once or twice to expel the fluorine, and the residue is dissolved finally in nitric acid and its solution added to the former.

To the solution obtained in either of the above ways ammoniummolybdate solution is added and the precipitate treated by one of the approved methods.

\section{FERROUS IRON.}

The presence of carbonaceous matter in limestones renders the exact or even approximate determination of ferrous iron often impossible. Nevertheless, even in its presence acceptable results are sometimes obtainable if there is not much of such matter and if it does not give with acid a colored solution. But even then it is only the soluble iron, existing chiefly if not wholly as carbonate, that is determinable. Occasionally limestones show films of manganese peroxide, which likewise interferes with the determination.

\section{A. IN ABSENCE OF CARBONACEOUS MATTER.}

a. FERROUS IRON SOLUBLE IN SULPHURIC ACID.

The powder, one to several grams, is introduced into a stout flask of about 200 to $250 \mathrm{~cm} \cdot{ }^{3}$ capacity and boiled with a little water till all air is expelled. While still boiling dilute sulphuric acid is added a 
little at a time, till effervescence ceases, and then a further amount. Calcium sulphate precipitates, but the iron will remain in solution. The flame is then removed and a stopper tightly inserted through which passes a small stopcock funnel. When cool or nearly so, cold water is poured into the funnel, the cock cautiously opened, and the water drawn into the flask, more water being poured into the funnel as fast as it empties, till the solution amounts to 100 or $150 \mathrm{~cm} .^{3}$. Such precaution to exclude air is hardly necessary in most cases, however, for in presence of sulphuric acid the oxidation of ferrous iron is exceedingly slow. It is ordinarily quite sufficient to equalize the internal and external pressures by opening the cock, removing the stopper, and pouring in cold water. The flask is then brought under a burette containing dilute permanganate solution and the iron titrated without delay. With a carbonate which is wholly decomposable without the aid of heat, this may be accomplished in a flask filled with carbon dioxide, whereby the danger of attack of silicates is lessened. If the preference is for the potassium-bichromate method of titration, hydrochloric acid may be used instead of sulphuric, in absence of manganese peroxide. In this case there is of course no separation of an insoluble calcium salt, a fact which renders easier the subsequent determination of the iron in any insoluble residue the rock may yield. The ferrous iron thus found is mostly, if not altogether, that existing as carbonate.

b. FERROUS IRON IN THE INSOLUB̨LE RESIDUE.

The titrated solution is filtered through a medium-sized hardened filter, the insoluble matter collected thereon and washed with water. The contents of the paper are rinsed into a capacious platinum crucible, the water mostly evaporated, and in the residue the ferrous iron is determined as in 19. C. d, page 140.

The total ferrous oxide found in a and $b$, when calculated to ferric oxide and subtracted from the total iron in the same state, gives the ferric oxide in the rock. If separate analyses have been made of the parts soluble and insoluble in dilute acid, similar calculations applied to the iron derived from the two portions will give the ferric oxide in each.

c. TOTAL FERROUS IRON.

If separate determinations are not desired, water is poured upon the powder in a capacious platinum crucible, dilute sulphuric acid is slowly added till effervescence ceases, then the crucible is placed in one of the forms of apparatus described in 19. C. d, page 140, together with $5 \mathrm{~cm} .^{3}$ of hydrofluoric acid, the decomposition of the silicates is effected in an atmosphere of carbon dioxide, and the titration is made as there directed. 


\section{B. IN PRESENCE OF CARBONACEOUS MATTER.}

Decomposition is effected in a flask by dilute sulphuric acid in an atmosphere of carbon dioxide. With limestones and active agitation no heat need be used, but with dolomites it will be necessary. The solution is then quickly filtered through asbestos (in an atmosphere of carbon dioxide if much iron is present), the residue and filter washed a few times, and the filtrate titrated at once with permanganate. If it is colored by organic matter, the result may be in error.

As in A. a, page 184, hydrochloric acid and titration by dichromate may be used in absence of manganese peroxide. In either case it is important to allow the acid to act no longer than is necessary and to filter quickly. A determination of the ferrous iron in the insoluble matter is usually not worth attempting, because of the admixed organic matter.

\section{ALKALIES.}

The alkalies in carbonate rocks are of course constituents of the siliceous components. Since these are usually subordinate it is advisable to operate on not less than 1 gram of the powder, and the J. Lawrence Smith method is to be followed in nearly all its details (p. 144). Because of the presence of a large amount of calcium carbonate in the rock it might seem that addition of precipitated carbonate would be needless. That it is not, however, appears to have been shown by experiments made with and without its addition. A little more alkali was obtained in the former case, a result that is probably due to more effective reaction of the finely divided artificial carbonate than of the crystalline natural carbonate with the ammonium chloride. One-half the usual amount of precipitated carbonate will suffice.

\section{CARBON DIOXIDE, CARBON (WATER).}

\section{A. DETERMINATION OF CARBON DIOXIDE.}

This determination is made precisely as under $21 . \mathrm{B}$, page 150 . If the absorption tubes have a height of about $10 \mathrm{~cm}$. and a diameter of $1.2 \mathrm{~cm}$. the first one will need refilling after every second analysis. For this reason Liebig or Geissler bulbs containing potash solution may be preferred.

\section{B. DETERMINATION OF CARBON OF GARBONACEOUS MATTER.}

The character of the carbonaceous matter in limestones has not been made the subject of study, so far as known to me, but it seems reasonable to assume that it resembles coaly matter rather than the indefinite humus. Although on solution of the rock in dilute acids 
the odor of the escaping gas is sometimes indicative of volatile organic substances, the amount thus escaping can never be more than a small fraction of the total. If the rocks are boiled with acid the filtrate may now and then be somewhat colored, but if care is taken to use dilute acid and to filter as soon as its action on the carbonates is over, little or no organic matter is likely to pass into solution. Hence, in all such cases the following method for the determination of its carbon is indicated, provided sulphides are practically absent.

Treat 1 to 10 grams of the rock with dilute hydrochloric acid till the carbonate constituents are surely decomposed, filter through an asbestos plug filling a constriction in a glass combustion tube, wash thoroughly with water, dry, and burn the carbonaceous matter over an ordinary burner in an air or oxygen current, collecting the escaping water in a calcium-chloride tube and the carbon dioxide in one filled with soda-lime. The combustion need occupy but a few minutes, as a rule.

If the rock is free from hydrous minerals, if the asbestos likewise yields no water on ignition, and if the asbestos and its load have been thoroughly dried, from the gain in weight of the calcium-chloride tube the hydrogen of the organic matter may be calculated. These conditions will, however, seldom be fulfilled, so that only the carbon has to be considered. In fact, it is quite useless, ordinarily, to attempt to determine the organic matter as such, either directly or by calculation. One must perforce be content with knowing its contained carbon.

C. SIMULTANEOUS DETERMINATION OF WATER AND OF TOTAL CARBON IN BOTH FORMS.

In presence of sulphides or soluble organic matter the following method will sometimes answer for the simultaneous determination of the first two or even all three of the above enumerated constituents.

Ignite in a current of air 1 gram of the rock in a tube of very hard glass, or in the tubulated crucible of Gooch (fig. 15, p. 63), collecting the water and the carbon dioxide as in B. The sulphur of sulphides will be retained as calcium sulphate. It is necessary for the success of this operation that the temperature be sufficient to drive out all cąrbon dioxide from the carbonates, but insufficient to decompose calcium sulphate. The water found represents that in the minerals of the rock as well as that derived from the hydrogen of the organic matter.

A separate determination of the carbon dioxide (see A., p. 186) gives the data for calculating the carbon of the organic matter.

According to G. T. Morgan ${ }^{a}$ the two conditions of carbon can be successively determined on the same portion of sample by first finding 
the carbon dioxide as in $A$, but with substitution of orthophosphoric: for hydrochloric acid, whereupon, after weighing and replacing the absorption tubes, chromic acid is added and the boiling renewed to oxidize the organic matter.

\section{CHLORINE.}

Several grams of the rock are dissolved in the cold, or with as low a heat as may be, in dilute nitric acid free from chlorine, the solution is filtered, silver nitrate added, and the precipitate collected and treated as usual. (See 22. A, B, and C, pp. 153-154.)

If the rock is likely to contain chlorine-bearing silicates, it is decomposed with its own weight or less of chlorine-free sodium carbonate in a platinum crucible over the burner or moderate blast without undue prolongation of the heating. The mass is extracted with hot water and the filtrate acidified with cold dilute nitric acid, or it may be decomposed directly with the acid and the solution filtered. The further treatment is as above given.

\section{FLUORINE.}

As with silicate rocks, an exact determination of fluorine in carbonate rocks is difficult if not impossible with our present methods, and little or nothing has been done along this line.

Because of the great preponderance of lime, direct fusion with sodium-potassium carbonate and silica is of somewhat doubtful practicability. Probably the following treatment will afford the best results.

Dissolve the powder in dilute acetic acid, as far as this may be possible without boiling, and filter. To the filtrate add solution of sodium carbonate till in some slight excess, boil, and treat the precipitate of calcium carbonate and possible fluoride as on page 157. Most of the fluorine that may have been dissolved by the acetic acid will thus be found. The siliceous matter insoluble in acetic acid is fused with sodium-potassium carbonate and further treated as in 23. B. a, page 155 .

\section{SULPHUR.}

\section{A. TESTS AS TO ITS CONDITION. DETERMINATION OF SULPHATE SULPHUR.}

In very many, perhaps most, carbonate rocks any sulphur present is chiefly if not wholly in the sulphide condition, and then usually as pyrite. In what condition it may be is readily ascertainable. If moistened lead-acetate paper is blackened when held over the mouth of a test tube in which some of the rock powder is being boiled with 
dilute hydrochloric acid, a soluble sulphide is indicated, but it is seldom more than a negligible trace. If the filtrate after approximate neutralization with ammonia gives a precipitate with barium chloride there is sulphate present. If the total sulphur obtained as in B, below, is in excess of that in the sulphate and soluble-sulphide states, the difference may safely be calculated to iron disulphide.

In the presence of much sulphide and also of sulphate sulphur the extraction of the latter should be made in an atmosphere of carbon dioxide, but usually this precaution is quite unnecessary.

\section{B. DETERMINATION OF TOTAL SULPHUR.}

a. METHODS OF CONVERTING SULPHIDES TO SULPHATES.

$\alpha$. By ignition without flux.-In a rock that is not heavily charged with sulphides the following method of my own has afforded excellent results, even in the presence of 1 per cent of organic matter, though its range of application has not been ascertained.

The platinum crucible containing 1 or 2 grams of the rock powder is placed in the perforated disk (fig. 3, p. 30) and exposed to the heat of a burner for fifteen minutes or to that of an inclined and moderate blast for ten minutes. So far as present experience teaches, it is not at all necessary to begin with a gentle heat. All sulphide sulphur is oxidized and retained by the lime as sulphate without any loss by volatilization, all organic matter is removed, and the silicates are rendered soluble in acid if they are not in excesș. (See 32. A. b. $\alpha$, p. 175.)

$\beta$. By ignition with sodium carbonate.-One to 2 grams of powder are mixed with half the weight of sulphur-free sodium carbonate in a platinum crucible and heated in the perforated disk, as in $\alpha$. It is not probable that addition of niter is called for, even when a good deal of carbonaceous matter is present. Since not enough flux is used to produce more than a sintering, the air entering the crucible after the bulk of the carbon dioxide has passed off effects very speedy oxidation in the porous mass.

With highly impure limestones it may be necessary at times to increase the amount of flux and to use a little niter as well.

b. TREATMENT AFTER IGNITION.

The ignited mass obtained in a, $\alpha$ or $\beta$, will usually separate easily from the crucible. It is transferred to a small beaker and covered with water. The crucible is cleansed with dilute hydrochloric acid and the solution poured into the beaker. More acid is added till decomposition is complete in the cold or on gently warming. The solution is then filtered, diluted if need be to 150 or $200 \mathrm{~cm}^{3}$, brought to boiling or simply placed on the steam bath, barium chloride added, and the precipitated sulphate collected in due time and weighed as usual. 
Evaporation to dryness to separate the dissolved silica is quite unnecessary. After decomposition with acid the solution should never be permitted to gelatinize, and it needs but little practice on the part of the operator to insure unfailing success in preventing this. If it should happen, however, it is generally best to begin over, but the difficulty may be overcome if but little free acid is present by dissolving the gelatinized silica in sulphur-free fixed alkali and reacidifying in the cold with hydrochloric acid.

44. WATER.

\section{A. HYGROSCOPIC WATER.}

From one to several grams of the air-dry powder are heated in a current of air dried by calcium chloride in a glass tube at 100 to $105^{\circ}$ and the escaping water coilected in a calcium-chloride tube, or the air may be dried by sulphuric acid and the water collected in sulphuric acid. This direct determination of the water is certain to give a higher and more correct result than that obtained by drying in a crucible at the above temperature.

\section{B. COMBINED WATER.}

This may be ordinarily determined on the air-dry sample by heating in a tube of combustion glass and collecting the water in a calciumchloride tube with the above-mentioned precautions. Or the powder may be mixed with dry sodium carbonate and heated in the apparatus of Gooch (5. C. b, p. 62) or with anhydrous borax according to Jannasch (5. C. d, p. 66). From the result thus found the separately determined hygroscopic water is to be deducted. It is to be remembered that the result will be in error by the amount of water afforded by the hydrogen of any organic matter there may be in the limestone.

\section{PART III.-CONDENSED ANALYSIS.}

For many purposes, especially those with a technical end in view, a highly refined or detailed analysis is not called for. The analyses of this kind that are now made vastly exceed in number those demanding the kind of work provided for in many of the sections of Part II. To meet the needs of those having work of this kind to do, a procedure is here given in brief, that covers the important constituents of limestones. The operations are generally given without explanations. Those who seek reasons for one or another procedure or details of manipulations are referred to the corresponding sections of Part II. 


\section{DECOMPOSITION AND SOLUTION.}

If the substance is convertible by blasting into a condition wholly decomposable by hydrochloric acid, one-half to 1 gram of the fine powder is strongly ignited in a covered platinum crucible over the blast for fifteen minutes, or longer if the blast is not very powerful.

If the material is very impure, one-half to $1 \mathrm{gram}$ is to be mixed with half its weight of pure sodium carbonate and ignited over the burner and blast till well sintered together.

The ignited material obtained in either of the above ways is transferred to an evaporating dish, preferably of platinum, and moistened with a few cubic centimeters of water. The crucible is cleaned with dilute hydrochloric acid, the solution poured into the dish, which is to be kept covered if sodium carbonate was used, and more acid added. Solution of the mass may be aided by gentle pressure with the flattened end of a glass rod. The solution is then evaporated to dryness on the steam bath. It will do no harm with limestones low in magnesium to hasten dehydration of the silica by placing the dish with its dry contents in an air bath at an elevated temperature or on a triangle resting on a hot plate at $200^{\circ}$ for an hour or less.

\section{SILICA.}

The residue is treated with 5 to $10 \mathrm{~cm} .^{3}$ of strong hydrochloric acid, which is then diluted to half strength, or upon the residue may be poured at once a larger volume of acid of half strength. The dish is then covered and digestion allowed to go on for ten minutes on the bath, after which the solution is passed through a 7-cm. paper and the silica washed with water, cold at first and hot later, or with hot dilute hydrochloric acid at first, followed by a little hot water. The filtrate is again evaporated to dryness, this time only on the steam bath, after which the residue is digested with acid as before, but in smaller amount, and the second portion of usually dark-colored silica is collected on another small paper. The filter papers with their contents are slowly charred in a platinum crucible, then ignited, first over the burner, finally over the blast for five to fifteen minutes, according to the amount of the silica. If the blast is weak, a second application of it should follow the first weighing. The finally weighed silica is moistened with water and two drops of sulphuric acid and 5 to $10 \mathrm{~cm}^{3}$ of pure hydrofluoric acid are added, the crucible is placed in a radiator (fig. 2, p. 29), and the liquid wholly evaporated. The residue is blasted for a minute and in due time weighed. It is better not to make this correction if it is not intended to separate and determine also the silica always accompanying the iron and aluminum oxides, but it should never be omitted if this latter correction is contemplated. 
47. ALUMINUM, IRON, ETC.

The filtrate, which need not exceed $100 \mathrm{~cm} .^{3}$ in bulk with fairly pure limestones, is brought to boiling in a beaker, or preferably in platinum, and made alkaline with ammonia after adding a few cubic centimeters of bromine water and if need be enough hydrochloric acid to insure a total of 10 to $15 \mathrm{~cm} .^{3}$ of strong acid. The ammonia should not be in such excess as to require long boiling to expel the most of it, nor is the expulsion of the whole of it necessary or indeed desirable. As soon as the precipitate settles the solution is passed through a filter of suitable size and the precipitate is washed a few times with hot water and sucked fairly dry. It is redissolved in hot hydrochloric acid and precipitated again by ammonia in presence of bromine, but with less ammonium chloride and in a smaller bulk of solution. If the filter is still in good condition, it is used also for this precipitate, which is washed with hot water and sucked dry at the pump. With high iron and magnesium the precipitation may well be repeated.

The re-solution of the first precipitate may be made with nitric acid if thought desirable. Its use involves very little washing of the second precipitate. To reduce the amount of aluminum passing into the filtrate, the precipitated oxides may be washed with water containing in the liter $20 \mathrm{~cm} .^{3}$ of nitric acid that has been neutralized with ammonia.

The paper containing the final precipitate is placed in a platinum crucible, slowly charred, then ignited over a gradually increased flame, and finally over the blast for two to five minutes. The weight found represents the oxides of ferric iron, aluminum, titanium, phosphorus, and manganese, the last presumably $\mathrm{Mn}_{3} \mathrm{O}_{4}$.

The iron, titanium, and silica in the ignited oxides are determined, after fusion with potassium or sodium pyrosulphate, exactly as detailed in 33.C, p. 180. The phosphorus and manganese, having been determined in different portions (37, p. 184, and 34. B, p. 182), the aluminum oxide is found by subtracting all these oxides from the original weight of the mixture. Often the percentage reported as alumina includes in reality the titanium, phosphorus, and some of the manganese, where no attempt has been made to separately determine these.

\section{CALCIUM.}

The combined filtrates from the iron, aluminum, etc., are concentrated if need be to a bulk of 200 to $250 \mathrm{~cm}^{3}$, with occasional addition of a few drops of ammonia. If a slight precipitate separates, it is collected on a small filter, washed with water, redissolved in hydrochloric acid, and reprecipitated by ammonia. It is added to 
and treated with the main quantity of iron and aluminum oxides, and the filtrates are combined. To them is added while boiling $20 \mathrm{~cm} .^{3}$ of a saturated solution of ammonium oxalate, and the boiling is continued until the precipitated calcium oxalate assumes a well-defined granular form. It is then allowed to stand for an hour, filtered, and washed a few times with hot water. The precipitate and filter are ignited in platinum till the oxalate is converted to oxide, which is redissolved in hydrochloric acid and the solution made up to $100 \mathrm{~cm}^{3}$. Ammonia is added in slight excess to the boiling solution and also ammonium oxalate to reprecipitate the lime. After an hour the oxalate is collected, washed a few times with small amounts of hot water, ignited over the blast, and weighed as the oxide.

Instead of igniting and weighing as oxide it is permissible to dissolve the oxalate in sulphuric acid, taking care to extract the filter thoroughly with hot dilute acid, and to titrate the solution with permanganate.

\section{MAGNESIUM.}

To the combined filtrates from the calcium precipitations are added sodium-ammonium phosphate solution in excess and, when the precipitate has formed after vigorous stirring, ammonia in considerable excess. After several hours, twelve or fifteen if the precipitate is very slight, the solution is filtered, the precipitate redissolved in hydrocloric acid and reprecipitated by ammonia in moderate excess and a drop of the phosphate precipitant in the usual manner. After two hours the precipitate is filtered on paper or on a Gooch filter, ignited, and weighed as pyrophosphate.

See 39, p. 186.

50. ALKALIES.

See 40, p. 186.

51. CARBON DIOXIDE.

See 43, p. 188.

52. SULPHUR.

53. WATER.

See 44, p. 190.

54. IGNITION LOSS.

This determination has little significance, though it affords a more or less correct indication of the proportion of carbonates in the rock. Since it is a determination commonly made in commercial laboratories, some space will be devoted to it.

Loss on ignition with carbonate rocks represents, as with those of a silicate nature, the algebraic sum of a number of chemical changes involving both losses and gains, and its amount will depend largely on the temperature employed.' At the temperature of a moderate

Bull. 305--07--13 
blast, with crucible covered, carbon dioxide, water, and carbonaceous matter escape wholly. Sulphides are oxidized to sulphates and all the sulphur is retained as sulphate by the calcium. With a powerful blast the sulphate is decomposed gradually, with eventual loss of the entire sulphur. At this point the alkalies begin to escape as oxides, the potassium relatively faster than the sodium, and they may be found in part condensed on the lid of the crucible. If the heating is long continued they can be wholly volatilized. As a small offset to these losses the iron of pyrite and the iron and manganese of ferrous and manganous carbonates take up oxygen and unite with the lime. By proper regulation of the temperature it is not difficult to prevent loss of sulphur and alkalies, and this is the object to be sought in making the determination.

One-half to 1 gram of the powder is placed in a platinum crucible of 20 to 25 grams weight, the crucible is inserted to three-fifths of its depth in a perforated platinum disk or asbestos board (fig. 3, p. 30), and an inclined blast flame of considerable power is caused to play against the bottom of the covered crucible for fifteen or twenty minutes. A repetition of the heating for five minutes will usually cause little or 'no further loss. Duplicate determinations should agree within 0.2 per cent, and after the operator becomes skilled probably within 0.1 per cent. A muffle can be substituted for the blast. In my experiments the crucibles occupied a position 2 inches inside the closed door of the muffle. It is, however, important that each operator should ascertain just what the proper conditions may be for his particular blast or muffle. They are right if after the ignition he finds exactly the whole of the sulphur in the ignited powder in the sulphate state. 


\section{N D E X .}

A. Page.

Absorption tuhes, use of, in water determination ..................... 61-68

Acids, quality of.......................... 35

Acknowledgments to those aiding .......... 13

Alkalies, determination of ........... 144-150, 186 determination of, apparatus for, figure showing..................... 145

Allen, E. T., cited..................... 95-96

Allen, E. T., and Day, A. L., cited........ 55 pycnometer method of .............. 43-45

Aluminum, concealment of, by manganese. $\quad 96$ direct determination of . ............. 93-96 indirect determination of... 81-90,177-181, 192 ignition of precipitate of oxide of ...... 87-88 precipitation of......... 82-86,95, 178-179, 192 recovery of, from filtrates..... 86-87, 179-180 separation of, from iron............. 93-95

Aluminum chloride, loss of ........ 83 (footnote) Ammonia, occurrence of, in rocks........ 163-164 Ammonium" chloride, reagent, preparation of . ..................... 82,144

Ammonium oxalate, reagent, quality of.... 36 Analysis, allowable error in ............. 25-26 completeness and thoroughness of, importance of ................ 14-20,38 crushing of rock for................. 45-47 diversity of, for same rock........... 16-17 grinding rock for................. 47-50 methods of. ....................... 12 neglect of certain factors in, consequences of . ................ 17-19

preparation of sample for........... 45-50 quantity of rock required for.. $20,37-38,45,50$ statement of ....................... 27 time required for................... 28 See also Carbonate rocks; Silicate rocks. Analysis, qualitative, usefulness of......... 34 Appliances and apparatus, descriptions and figures of .................. 28-34

Aragonite, tests for....................... 72

Atterberg, A., cited .............. 147 (footnote)

Austin, M., and Gooch, F. A., cited. 98, 105-106, 122

\section{B.}

Bailey, G. H., cited .... B.

Barium, determination of. 102-103,116-118, 180, 183 ignited silica free from $\ldots$ precipitability of, by ammonium persulphate.....................

s. recovery of, from alumina precipitate removal of, from magnesium pyrophosphate.

separation of, from strontium ......... 103
Baskerville, C., method of, in determination of titanium................ 115 method of, in determination of zirconium............. 120

Baubigny, H., method of, in determination of chromium ................. 124 Bibliography of rock analysis............... 11-12 Bisbee, H., Richards, T. W., and McCaffrey, C. T., cited.................. 101 Blair, A. A., crucible tongs of ............. 28 Blast, temperature of, test of ...... 79 (footnote) Blount, B., cited..................... 78, 177 Bolm, F., cited................. 147 (footnote) Bolton, H. C., cited ........................ 172 Boric oxide, reagent, preparation of ........ $\quad 70$ Boron, determination of................ 161-162 occurrence of, in rocks................ 25 Broockmann, K., cited................... 108 Browning, P. E., cited................... 102 Brühl, E., and Friedheim, C., cited....... 97,124 Brunck, O., cited ...................... 104,105 basic-acetate method of, in separation of iron, manganese, etc... 85 (footnote)

Brush, G. J., method of, in water determination ......................... 59-60

Buckley, E. R., method of, for determination of porosity .............. 40-41

Bunsen, R., cited 55,75

\section{C.}

Calcite, tests for................... 150,172,173 Calcium, determination of .... 101, 105, 183, 192-193 ignition of oxalate of............... 101-102 occurrence of, in rocks............ 169-170 precipitability of, by ammonium persulphate.................... removal of, from magnesium pyrophosphate ..................... 109-110

in alkali determination............ 146 separation of strontium from .......... 102 Calcium chloride, reagent, deterioration of . $\quad 56$ Calcium fluoride, testing of precipitate of. 157-158 Calcium oxalate, solubility of ............. 101 Cameron, $\Lambda$. , cited....................... 75 Campbell, E. D.. and Hess, W. H., cited... 95 Carbon, determination of............ 153, 186-188 effect of, on ferrous-iron determination. 138 , $140,184,186$ occurrence of in rocks ............. 171

Carbonate rocks, analysis of........... 167-194 analysis of, defects of, as usually made $168-170$ condensed forms of ............ 190-194 detailed methods of .............. 174-190 causes of color of .................. 170 
Page.

Carbonate rocks, ignition loss of......... 193-194 importance of ................... 12 impurities in .................... 167-168 mineral composition of ............ 169-171 qualitative comparison of silicate rocks and ...................... 167-168 qualitative tests of . . . . . . . . . . . . . 171-173 Carbonates, qualitative tests of . ........ 171-173

Carbon dioxide, direct determination of .... 150

direct determination of, apparatus for, figure showing.............. 151 expulsion of, by heat............... 163 indirect determination of . ........... 152-153

Carbon monoxide, evolution of, on heating rocks......................... 163

Carnot, A., cited....................... 92 Cathrein, A, cited .. 93 (footnote), 113 (footnote)

Cerium, detection of . . .................. 121 occurrence of, in piedmontite... 83 (footnote)

Chalcopy rite, occurrence of, in rocks....... 24

Chatard, T. M., cited................ 113, 123 drying oven of, description and figure of . 57-58

Chlorine, condition of, in rocks........... 154 determination of ............. 153-155, 188 occurrence of, in rocks................ 24

Chromium, colorimetric determination of. 116$117,123-125,127-131,182-183$ colorimetric determination of, comparison of gravimetric method and. 125-126

effect of vanadium on............ 123 gravimetric determination of.......... 124 occurrence of, in rocks.............. 22, 131

Citric acid, relative solubility of carbonates in........................ 172

Claasen, Edo, cited.................. 127

Clarke, F. W., cited .................. 18

Classen, A., cited..................... 36

Cloedt, E. v., and Jannasch, P., cited...... 97, 124

Cobalt, detection of . ................. 97-98 determination of . ............. 98, 182-183

occurrence of, in rocks............... 23

Colorimeters, description of and figures showing................... 31-34

Colorimetric methods, for determination of chromium......... 124-125, 127-129

for determination of chromium, comparison of gravimetric method and $\ldots \ldots \ldots \ldots \ldots \ldots \ldots \ldots \ldots . .125-126$

for determination of manganese...... 99-100 of titanium . $\ldots \ldots \ldots \ldots \ldots \ldots, 110-112,116$ of vanadium.

Columbium, occurrence of, in rocks......... 25

Cooke, J. P., method of, for determination of ferrous iron.......... 135, 140-142 method of, apparatus for, figure showing........................ 141

Coppadoro, A., cited ............ 90 (footnote)

Copper, determination of.......... 98-99, 182-183 occurrence of, in rocks............... 23

Cramer, E., cited .......................

Cross, W., and others, cited............... 38

Crucible, disk for, description and figure of . 30-31 tongs for, description and figure of...... 28-29

Crucibles, platinum, loss of weight of.. 78 (footnote), 88 (footnote)
Page.

Crushing, methods of ............ $45-47$

Crystal water. See Water.

Curtis, J. S., cited ....................... 162

Czudnowitz, C., cited................. 127

D.

Daniel, K., cited............... 156 (footnote

Davis, J. F., cited..................... 120

Davy, Humphrey, cited.................. $\quad 70$

Day, A. L., and Allen, E. T., cited......... 55 pycnometer method of .............. 43-45

Delesse, A., cited . ...................... 163

Desiccators, use of . . . . . . . . . . . $51,52,53,56$ Determinations, importance of order of... 37

Deussen, E., cited........... 89 (footnote), 136 Dieulafait, L., cited......... 18, 20 (footnote), 163 Dittrich, Max, cited................... 11, 26, $50,67,72,83,88,95,149$ method of, in determination of titanium.................... 115-116

Dittrich, Max, and PohI, R., cited ....... 115, 120

Dolomite, definition of ................ 168 tests for........................... 173

Drying before analysis, uselessness of..... 50 Drying for hygroscopic tests, process of... 50-52 Dunn, J. F., cited .............. 160 (footnote) Dunnington, F. P., cited............... 111

E.

Eakins, L. G., cited.......... 42-43 Electrification of glass tubes, influence of, on weight................. 50

Ellis, C. W. H., mortar of, description and figure of .................. 46

Erdmann, H., cited....................... 164 Error in analysis, allowable limits of..... 25-26 F.

Feldspars, error in calculation of ........ 18 Ferric iron, calculation of ............... 93 See also Iron.

Ferrous iron, determination of ... 131-144, 184-186 determination of, hydrofluoric-acid method of ............... 135-144 sealed tube method of.......... 133-135 See also Iron.

Fischer, T., cited . ................... 131

Fluid inclusions, volatilization of......... 163

Fluorine, determination of......... 155-159, 188 influence of, in silica determination.... 68 , 71 (footnote) occurrence of, in rocks............... 24-25 qualitative tests for............... 155 Forchhammer, J. G., cited............... 163 Ford, W. E., cited..................... 162 Foullon, H. von, cited. ................. 162 Frantz, B., and Streit, G., cited........ 119-120 Fresenius, R., cited......... 101 (foot note), 103 Friedel, C., cited....................... 52

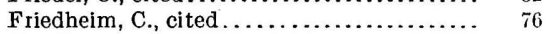
Friedheim, C., and Bruhl, E., cited........ 97, 124

Funk, W., basic-acetate method of, for separation of iron, manganese, etc............... 85 (footnote)

G.

Galena, occurrence of, in carbonate rocks... 170 Gases, evolution of, on heating rocks..... 163-164 
Page.

Gautier, A., cited..................... 163

Gibbs, Wolcott, cited.................. 106

Gilbert, J. P., cited . ............... 75-76, 177 Glassware, inferiority of....... 31 (footnote), 35 Glucinum, occurrence of, in rocks........ 25

Gold, occurrence of, in rocks............

Gooch, F. A., apparatus of, for water determination, description and figures of . . . . . . . . . . . . . . . 62-65

cited ................. 93, 161 (footnote) method of, in determination of lithium. 148

in determination of titanium. . ... 113-115

Gooch, F. A., and Austin, M., cited. 98, 105-105, 122 Gooch, F. A., and Jones, L. C., cited

161 (footnote)

Grinding, fineness of. methods of $47-49$

\section{H.}

Hall, R. W., cited................... 78

Harper, D. N., and Penfield, S. L., cited.... 82

Harrington, B. J., cited................. 171

Harrison, J. B., cited..................23, 25

Hauynite, evidence as to presence of . ..... 159 occurrence of, in rocks............... 24

Hayes, A. A., cited.................... 18

Heidenreich, O., and Jannasch, P., boricoxide method of, for decomposin silicates..................

Hempel, W., cited.

Hewett, Foster, cited....................

Hess, W. H., and Campbell, E. D., cited ...

Hinden, F., cited.

Holthof, C., cited. . . .................. 72,74

Horn, D. W., cited.................... 125

Hutchinson, A., cited.................. 172

Hüttner, K. cited.................... 163

Hydrofluoric acid, reagent, quality of..... 35

Hydrofluoric-acid method of determining ferrous iron ................ 135-144

Hydrogen, evolution of, on heating rocks .. 163

Hydrogen peroxide, reagent, quality of ... 36,111

Hydrogen sulphide, occurrence of, in carbonate rocks.................. 171

Hygroscopic water, determination of. $50-58,66,190$ See also Water.

$$
\text { I. }
$$

Iddings, J. P., and others, cited......... . . 38 Ignition, loss by carbonate rocks on..... 193-194 loss on, inaccuracy of water determination by

temperature of, variations in (footnote)

Iron, correction of titanium determination

for .................... 112-113 determination of .... 90-93, 131-144, 177-181, 192 effect of carbonaceous matter on.... 138 , $140,184,186$

effect of sulphides on ...132-133, 138-140, 161 effect of vanadium on ........ 91-93,140 ignition of precipitate of oxide of ..... . 87-88 occurrence of, in carbonate rocks..... 169,170 oxidizability of divalent, by free oxygen, in presence of a fluoride........ 137 precipitation of ............ 82-86, 178-179, 192 recovery of, from filtrate............ 86-87 reduction of . . . . . . . . . . . . . . . . . .
Fage.

Iron, separation of, from aluminum. ..... 93-95

in titanium determination........ 113-114 solution of oxide of, by alkali fusion.... 93 titration of . .......... 91-92,142-144, 181, 184 See also Ferric iron; Ferrous iron.

Iron oxides, error in determination of..... 19, $131-133,135,138-140,144$

J.

Jannasch, P., Nickel-becher of . . . . . . . 29 silicate decomposition methods of...... C,9-70 water determinations of, apparatus for, figure of................... 67 method of $\ldots \ldots \ldots \ldots \ldots \ldots \ldots \ldots \ldots, 66-68$ work of Jannasch, P., and Cloedt, E. v., cited.... 97,124 Jannasch, P., and Heidenreich, O., boricoxide method of ............ 70-71 Jannasch, P., and Weber, H., cited

71 (footnote)

Jannettaz, E., cited ................. 172

Järvinen, K. K., cited................ 106, 108 Jones, L. C., and Gooch, F. A., cited

161 (footnote)

K.

Knop, A., cited................. 90 (footnote) Koch, A. A., and Treadwell, F. P., cited. . 157, 158 Koninck, L. L. de, cited . . . . . . . . . . . . . . 108, 132 Koningh, L. de, cited.......... 124 (footnote) Krauch, C., cited...................... $\quad 35$

Kreider, J. L., apparatus of, for determination of volatile substance, description and figure of ........ 152-153

I.

Lang, W. R., and Manning, R. J., cited 161 (footnote)

Lazurite, evidence of presence of, in rocks.. 159 occurrence of, in rocks................ 24

Lead, occurrence of, in rocks............ 25

Lemberg, J., cited . . . . ..................... 173

Limestones, causes of color in . . . . . . . . . $\quad 170$ minor constituents of . ............. 169

Limestones, dolomitic, definition of ....... 168 Limestones, magnesian, definition of ....... 168

Lindemann, 0 ., cited ................. 127

Lindo, D., cited........................ $\quad 76$

Literature of analysis. . . . . . . . . . . . . . 11-12

Lithium, determination of . . . . . . . . . . 147-148 occurrence of, in rocks................ 24

Low, W. H., cited............. 161 (footnote)

Löwe, J., clay disks of ................... 31

Ludwig, ${ }^{2} .$, cited....................... $\quad 75$

Luedeking, C., cited..................... 164

Lunge, G., cited......................... $\quad 31$

Lunge, G., and Millberg, C., cited..... 78, 165-166

M.

McCaffrey, C. T., Bisbee, H., and Richards, T. W., cited. . . ................

McKenna Brothers Brass Cos, ore grinder of, description and figure of . . . 47-48 Magnesium, determination of. .... 105-110, 183, 193 occurrence of, in carbonate rocks...... 169 
Page.

Magnesium, precipitate of, ignition of .... 108-109 precipitate of, contaminants of, removal of ...................... 109-110 precipitation of.

Maillard, L. C., cited . . . . . . .............. 131 Manasse, 0. , cited ........................ 128

Manganese, determination of .......... 96-100, $110,181-182,192$ occurrence of, in rocks.............. 23, 169 oxidizability of divalent, in presence of hydrofiuoric acid........... 135-137 precipitation of . ........ 85-86, 97-99, 178-179 removal of, from magnesium pyrophosphate......................

Manning, R. J., and Lang, W. R., cited....

Mar, F. W., cited

Marble, contamination of, by sulphides 91 (footnote)

Meade, R. K., cited ................... 176

Mechanical separation, literature of . ...... 12-13

Meigen, W., cited...................... 172

Meineke, C., cited ....................... 75

Meteorites, analysis of................ 12

Microscopic examination, value of........ 19-20

Millberg, C., and Lunge, G., cited . ..... 78, 165-166

Minerals, specific gravity of fragments of ... 42

Mingaye, J. C. H., cited ........... 19 (footnote)

Minor, J. C., and Penfield, S. L., cited....... 157

Mitchell, W. L., and Wells, H. A., cited 91 (footnote)

Mitscherlich method of ferrous-iron determination............... 131, 133-135 objections to ........ 131, 133,133 (footnote)

Mittasch, A., basic-acetate method of. for separation of manganese 84-85 (footnote)

Moissan, H., cited....................... 161

Molybdenum, determination of...... 128,182, 183 occurrence of, in rocks........ 19,25, 126,170

Moore, E. G., device of, to remove air from mineral powders, description and figure of ................ 44-45

Morgan, G. T., cited.............. 153,187-188

Morley, E. W., cited............ 145 (footnote) Moureu, C., cited..................... 163

N.

Nepheline, detection of, in presence of olivine ................... 164-165

Neubauer, H., cited............... 105-106, 108

Neustadl, I.., and Skrabal, A., cited........ 103

Nickel, determination of ............ 98, 182-183 occurrence of, in rocks.............. 23,170 precipitation of ..................... 97-99

Nickel-becher, description of...

Nitrogen, occurrence of, in rocks....

Noselite, evidence as to presence of.......... 159 occurrence of, in rocks ................ 24

Noyes, W. A., cited...................... 112-113

O.

Organic matter, removal of..

Oxidation, incompleteness of, on ignition of ferrous compounds.
P.

Page,

Panebianco, G., cited ......... 172

Pattinson, I., cited ..................... 160

Penfield, S. L., cited . . . . . . . . . . . . . . . . . . . 42,82 method of, for water determination..... 59-62 apparatus for, figures showing ......60,61

Penfield, S. L., and Harper, D. N., cited... 82

Penfield, S. L., and Minor, J. C., cited...... 157

Penfield, S. L., and Sperry, C. S., cited 161 (footnote)

Peters. R., cited ....................... 137

Phenylhydrazine, reagent, contamination of ................ 95 (footnote)

Phosphorus, determination of.... 121-123, 184, 192 loss of, by volatization................ 94 occurrence of, in rocks ............... 24,171 precipitation of . .................. 178-179

Phosphorus salt, reagent, quality of...... . 36 Piedmontite, rare earths in....... 83 (footnote) Pinagel, A., cited ...................... $\quad 76$ Pirsson, L. V., cited . ................ 27,164-165 Pirsson, I. V., and others, cited........... 38 Platinum, occurrence of, in rocks...... 25,80-81 precipitation of ......... 81,90-91 (footnote) reduction of, from solution.... 147 (footnote)

Platinum crucibles, loss of weight in 78 (footnote), 88 (footnote)

Pohl, R., and Dittrich, Max, cited...... 115,120 Porosity, determination of ........... 38, 40-42 Potassium, determination of ............ 147 Potassium bisulphate, reagent, conversion of, to pyrosulphate........... 36 objections to use of ......... 88 (footnote) quality of . . . . . . . . . relative solubility of carbonates in..... $\quad 172$

Potassium pyrosulphate, reagent, advantages n use of...... 88-89 (footnote)

Powell, N. S., and Richards, J. W., cited.... 172

Pratt, J. H., method of, in ferrous-iron determination. .......... 137,142-143

Precht, H., cited.............. 147 (footnote)

Pycnometer method of specific-gravity determinations................. 42-45

Pyrite, effect of, on ferrous-iron determination...................... 138-140

occurrence of, in carbonate rocks....... 170

Pyrrhotite, evidence as to presence of, in rocks. 159

R.

Radiators for evaporating solutions....... 29 Ransome, F. . L., cited................... 22, 126 Rare earths, caution against overlooking of ............. 83 (footnote), 97 determination of. . 116-117, 119, 120-121, 182-183 occurrence of, in rocks............... 25 Reagents, quality of .................... $34-37$ testing of ......................... 35

Richa.rds, J. W., and Powell, N. S., cited.... 172 Richards, T. W., McCafirey, C. T., and Bisbee, H., cited .............. 101

Ridsdale, C. H., cited................... 92,130

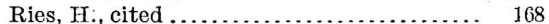
Riggs, R. B., method of, for separation of magnesium from alkalies... . . . 149-150 Robertson, J. D., cited . ................. 163 Rose, H., cited.. 94 (footnote), 156 (footrote), 163 Rosenblatt, Th., cited........... 161 (footnote) 
S.

Sandberger, F., cited......... 20 (footnote), 162 Sargent, G. W., cited . ....... 161 (footnote), 162 Schaffgotsch's solution, character of 149 (footnote) use of. 156 (footnote)

Schaller, W. T., cited............ 146 (footnote) Schreiner, Oswald, colorimeter of, nescription and figure of . . . . . . . . . 33-34 Scoop. See Weighing, scoop for.

Seemann, F., cited........ 156 (footnote), 158-159 Seger, H., cited

Separation, mechanical, literature of....... 12-13 Shimer, P. W., cited ................... 176 Sieves, metal, unavailability of........... $\quad 49$ Silica, dehydration of.................. $75-78$ determination of $\ldots \ldots \ldots \ldots \ldots .68-81$, $88-90,159,174-177,180,191$ accuracy of . ...... 79,89 impurities in, correction for........... 79 precipitate of, ignition of............ 78-79 recovery of, from alumina precipitate. . 88-90 removal of, in fluorine determination . 156 in sulphur determination .......... 160 residue from, composition of ......... 80 separation of, from alumina, etc. $68-81,176-177$ fluxes used in ..................68-75 methods of . . . . . . . . . . . . .

Silica, soluble, determination of ......... 165-166

Silicate rocks, analysis of ............... 14-166 analysis of, methods of . . . . . . . . . . 37-166 constituents in . ..................... 20-25 literature of ....................... 11 methods of decomposition of ......... 69-74

Siliceous limestones, character of......... 170

Silver, occurrence of, in rocks........... 25

Silvestri, O., cited . . . . . . .................. 164

Sjollema, B., cited ........................ 166

Skrabal, A., and Neustadl, L., cited....... 103 Smith, J. Lawrence, cited .......... 89 (footnote) method of, for alkali determination .. 144-145 apparatus for, figure showing...... 145

Sodium, determination of .............. 147

Sodium bisulphate, objections to . . .88 (footnote)

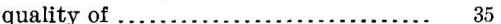

Sodium carbonate, reagent, quality_of..... 36, 72

Sodium hydroxids, reagent, quality of..... 36 Sodium-potassium carbonate, comparison of sodium carbonate and ...... 71-72

Sodium pyrosulphate, reagent, preparation

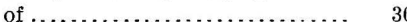

use of, advantages......... 88-89 (footnote)

Solutions, heavy, unavailability of, in specific gravity determinations of rocks...

Sonstadt, E., cited............. 147 (footnote)

Specific gravity, determination of . . . . . . . 38-45

Sperry, E. S., and Penfield, S. L., cited..... 161 (footnote)

Sphalerite, occurrence of, in carbonate rocks. 170

Steiger, George, cited. 96 (footnote), 146 (footnote) drying apparatus of, description and figure of................. $56-57$

Steinlen, R. L., cited . ........ 145 (footnote) Stoddart, C. W., cited . . . . . . . . 160 (footnote) Stokes, H. N., cited . . . . . . . . . . . 119, 132, 133, 139 Streit, G., and Franz, B., cited ......... 119-120 trontium, determination of ........... 102 occurrence of, in rocks............. 18,23-24 separation of, from calcium......... 102

from magnesium............... 101-102

Sulphides, conversion of, to sulphates in limestones .................. 189

Sulphur, condition of,determination of . 159, 188-89 determination of...... 116-117,159-161, 189-190 failure to determine, error arising from. $\quad 19$ occurrence of, in rocks................ 19,24 removal of, in alkali determination..... 146 Sulphur dioxide, reagent, quality of ....... 128 (footnote) T.

Tantalum, occurrence of, in rocks.......... Tartaric acid, relative solubility of carbonates in................... 172

removal of, in titanium determination.. 114 Thörner, T. W., cited.................. 42 Thorpe, T. E., cited . ................... 18 Titanic oxide, solubility of, in alkalies..... 94 Titanium, colorimetric determination of ... 110113,181

colorimetric determination of, superiority of . . . . . 116 gravimetric determination of.. 94,113-116, 192 loss of . . . . . . . . . . . footnote) occurrence of, in rocks.............. 21-22 precipitation of... 81,90 (footnote), 93,178-179 Titanium sulphate, reagent, preparation of. 111 (footnote)

Toluene bath, description and use of . . ... 51,56 Trace, definition of................... 27 Travers, M. W., cited.................. 163

Treadwell, F. P., cited................... 78 method of, for ferrous-iron determination ..................... 135, 143-144

apparatus for, figure showing..... 143 Treadwell, F. P., and Koch, A. A., cited.. 157-158 Tungsten, occurrence of, in rocks......... 25 Tyrolite, drying of, experiment on....... 51-52

$\mathrm{U}$.

Ulbricht, R., cited . .................. 108-109 Ullmann, C., cited...................... 134 Uranium, occurrence of, in rocks ......... 25

V.

Vanadium, colorimetric determination of . . 131 effect of, on iron determination. $92-93,126,140$ failure to determine, error from ......... 19 gravimetric determination of ......... 123, $127-131,182,183$ occurrence of, in rocks.... 18-19,22-23,126,171 Vogt, J. H. L., cited ....................... 132 Volatile substances, determination of, apparatus for, description and figure of . . . . . . . . . . . . Volhard, J., cited. . . . . . . . . . 98 (footnote) W.

Walters, H. E., cited............ 99 (footnote) Warwickite, analyses of, discordancy of... 162 Washington, H. S., cited ............... 11-12, 15 (footnote), 16 (footnote) , 20, 21, 23, $26,27,47,91$ (footnote), 96 (footnote) 
Washington, H. S., method of, in determination of phosphorus...... 121-122

Washington, H. S., and others, cited...... 38 Water, direct determination of . .... 50 -68, 187, 190 direct determination of, apparatus for, description and figures of...... 56-68 precautions to be observed in...... 56 indirect determination of . ...... 51-53,59-66 porosity and, determination of ........ 40-42 suspension in, determination of specific gravity by . . . . . . . . . . . . . $39-40$

Weber, II., horic-oxide method of, for decomposing silicates.......... 70-71

Weighing scoop, description and figure of Weller, A., method of, for determination of titanium................ 110-112

Wells, H. A., and Mitchell, W. L., cited.... 91 (footnote)

Wheeler, H. A., cited ................... 164 Whitfield, J. E., cited ................... 162
Page.

Williamson, J. A., cited.............. 3is Wülfing, E. A., cited............... 132, 149

Z.

Zeolitic minerals, dehydration of ........ 52 occurrence of, in rocks........ 39 (footnote) Zeolitic water. See Water.

Zimmerman, C., method of, for separation of magnesia................ 149

Zinc, determination of ............ 98, 182-18; occurrence of, in rocks.............. $\quad 25$ precipitation of . . . . . . . . . . . . . . . . 97-99 Zinc-oxide solution, preparation and use of. 156 (footnote)

Zirconium, determination of . . 94,116-117, 118-120 difficulty with, in gravimetric titanium determination

occurrence of, in rocks 


\section{CLASSIFICATION OF THE PUBLICATIONS OF THE UNITED STATES GE(OLOGICAL SURVEY.}

[Bulletin No. 305.]

The serial publications of the United States Geological Survey consist of (1) Annual Reports, (2) Monographs, (3) Professional Papers, (4) Bulletins, (5) Mineral Resources, (6) Water-Supply and Irrigation Papers, (7) Topographic Atlas of United States-folios and separate sheets thereof, (8) Geologic Atlas of the United Statesfolios thereof. The classes numbered 2,7 , and 8 are sold at cost of publication; the others are distributed free. A circular giving complete lists can be had on application.

Most of the above publications can be obtained or consulted in the following ways:

1. A limited number are delivered to the Director of the Survey, from whom they can be obtained, free of charge (except classes 2, 7, and 8), on application.

2. A certain number are delivered to Senators and Representatives in Congress for distribution.

3. Other copies are deposited with the Superintendent of Documents, Washington, D. C., from whom they can be had at practically cost.

4. Copies of all Government publications are furnished to the principal public libraries in the large cities throughout the United States, where they can be consulted by those interested.

The Professional Papers, Bulletins, and Water-Supply Papers treat of a variety of subjects, and the total number issued is large. They have therefore been classified into the following series: A, Economic geology; B, Descriptive geology; C, Systematic geology and paleontology; D, Petrography and mineralogy; E, Chemistry and physics; F, Geography; G, Miscellaneous; H, Forestry; I, Irrigation; J, Water storage; K, Pumping water; L, Quality of water; M, General hydrographic inyestigations; N, Water power; O, Underground waters; P, Hydrographic progress reports. This paper is the forty-ninth in Series $\mathrm{E}$, the complete list of which follows ( $\mathrm{PP}=$ Professional Paper; $\mathrm{B}=$ Bulletin):

\section{SERIES E, CHEMISTRY AND PHYSICS.}

B 9. Report of work done in the Washington laboratory during the fiscal year 1883-84, by F. W. Clarke and T. M. Chatard. $1884.40 \mathrm{pp}$. (Out of stock.)

B 14. Electrical and magnetic properties of the iron carburets, by Carl Barus and Vincent Strouhal. 1885. $238 \mathrm{pp.}$ (Out of stock.)

B 27. Report of work done in the Division of Chemistry and Physics, mainly during the year 1884-85. 1886. $80 \mathrm{pp}$.

B 32. Lists and analyses of the mineral springs of the United States (a preliminary study), by Albert C. Peale. $1886.235 \mathrm{pp}$. (Out of stock.)

B 35. Physical properties of the iron carburets, by Carl Barus and Vincent Strouhal. $1886.62 \mathrm{pp}$.

B 36. Subsidence of fine solid particles in liquids, by Carl Barus. 1886. $58 \mathrm{pp.} \mathrm{(Out} \mathrm{of} \mathrm{stock.)}$

B 42. Report of work done in the Division of Chemistry and Physics, mainly during the fiscal year 1885-86, by F. W. Clarke. 1887. 152 pp., 1 pi. (Out of stock.)

B 47. Analyses of waters of the Yellowstone National Park, with an account of the methods of analyses employed, by F. A. Gooch and J. E. Whitfield. 1888. 84 pp. (Out of stock.)

B 52. Subaerial decay of rocks and origin of the red color of certain formations, by I. C. Russell. 1889 . $65 \mathrm{pp} ., 5$ pls. (Out of stock.)

B 54. On the thermoelectric measurement of high temperatures, by Carl Barus. 1889. 313 pp., 11 pls. (Out of stock.)

B 55. Report of work done in the Division of Chemistry and Physics, mainly during the fiscal year 1886-87, by F. W. Clarke. 1889 . 96 pp. (Out of stock.)

B 60. Report of work done in the Division of Chemistry and Physics, mainly during the fiscal year 1887-88. $1890.174 \mathrm{pp.} \quad$ (Out of stock.)

B 64. Report of work done in the Division of Chemistry and Physies, mainly during the fiscal year 1888-89, by F. W. Clarke. 1890 . 60 pp. 
B 68. Earthquakes in California in 1889 , by J. E. Keeler. 1890.25 pp.

B 73. The viscosity of solids, by Carl Barus. 1891. xii, 139 pp., 6 pls.

B 78. Report of work done in the Division of Chemistry and Physics, mainly during the fiscal year 1889-90, by F. W. Clarke. 1891. $131 \mathrm{pp.} \mathrm{(Out} \mathrm{of} \mathrm{stock.)}$

B 90. Report of work done in the Division of Chemistry and Physics, mainly during the fiscal year 1890-91, by F. W. Clarke. 1892. 77 pp.

B 92. The compressibility of liquids, by Carl Barus. 1892.96 pp., 29 pls.

B 94. The mechanism of solid viscosity, by Carl Barus. 1892.138 pp.

B 95. Earthquakes in California in 1890 and 1891, by E. S. Holden. 1892. 31 pp.

B 96. The volume thermodynamics of liquids, by Carl Barus. $1892.100 \mathrm{pp}$.

B 103. High temperature work in igneous fusion and ebullition, chiefly in relation to pressure, by Carl Barus. 1893. 57 pp., 9 pls.

B 112. Earthquakes in California in 1892, by C. D. Perrine. $1893.57 \mathrm{pp}$.

B 113. Report of work done in the Division of Chemistry and Physics during the fiscal years 1891-9:2 and $1892-93$, by F. W. Clarke. 1893.115 pp.

B 114. Earthquakes in California in 1893, by C. D. Perrine. 1894. 23 pp.

B 125. The constitution of the silicates, by F. W. Clarke. 1895 . $100 \mathrm{pp}$. (Out of stock.)

B 129. Earthquakes in California in 1894, by C. D. Perrine. 1895.25 pp.

B 147. Earthquakes in California in 1895, by C. D. Perrine. $1896.23 \mathrm{pp}$.

B 148. Analyses of rocks, with a chapter on analytical methods, laboratory of the United States Geological Survey, 1880 to 1896 , by F. W. Clarke and W. F. Hillebrand. 1897. 306 pp. (Out of stock.)

B 155. Earthquakes in California in 1896 and 1897, by C. D. Perrine. 1898. 47 pp.

B 161. Earthquakes in California in 1898, by C. D. Perrine. 1899.31 pp., 1 pl.

B 167. Contributions to chemistry and mineralogy from the laboratory of the United States Geological Survey; F. W. Clarke, Chief Chemist. 1900.166 pp.

B 168. Analyses of rocks, laboratory of the United States Geological Survey, 1880 to 1899, tabulated by F. W. Clarke. 1900. 308 pp. (Out of stock.)

B 176. Some principles and methods of rock analysis, by W. F. Hillebrand. $1900.114 \mathrm{pp}$.

B 186. On pyrite and marcasite, by H. N. Stokes. 1900.50 pp.

B 207. The action of ammonium chloride upon silicates, by F. W. Clarke and George Steiger. 1902. $57 \mathrm{pp}$. (Out of stock.)

PP 14. Chemical analyses of igneous rocks published from 1884 to 1900 , with a critical discussion of the character and use of analyses, by H. S. Washington. $1903.495 \mathrm{pp}$.

PP 18. Chemical composition of igneous rocks expressed by means of diagrams, with reference to rock classification on a quantitative chemico-mineralogical basis, by J. P. Iddings. 1903. 98 pp., 8 pls.

B 220. Mineral analyses from the laboratories of the United States Geological Survey, 1880 to 1903 , tabulated by F. W. Clarke, Chief Chemist. $1903.119 \mathrm{pp}$.

B 228. Analyses of rocks from the laboratory of the United States Geological Survey, 1880 to 1903 , tabulated by F. W. Clarke, Chief Chemist. 1904. 375 pp.

PP 28. The superior analyses of igneous rocks from Roth's tabellen, 1869 to 1884, arranged according to the quantitative system of classification, by H. S. Washington. $1904.68 \mathrm{pp}$.

$\mathrm{B} 239$. Rock cleavage, by C. K. Leith. 1904. 216 pp., $27 \mathrm{pls}$.

B 241. Experiments on schistosity and slaty cleavage, by G. F. Becker. 1904. 34 pp., 7 pls.

B 253. Comparison of a wet and crucible-fire method for the assay of gold telluride ores, with notes on the errors occurring in the operations of fire assay and parting, by W. F. Hillebrand and E. T. Allen, 1905. $33 \mathrm{pp}$.

B 261. Preliminary report of the operations of the coal-testing plant of the United States Geological Survey at the Louisiana Purchase Exposition, St. Louis, Mo., 1904; E. W. Parker, J. A. Holmes, M. R. Campbell, committee in charge. 1905. $172 \mathrm{pp}$.

B 262. Contributions to mineralogy from the United States Geological Survey, by F. W. Clarke, W. F. Hillebrand, F. G. Ransome, S. L. Penfield, Waldemar Lindgren, George Steiger, and W. T. Schaller. 1905. $147 \mathrm{pp}$.

PP 48. Report on the operations of the coal-testing plant of the United States Geological Survey at the Louisiana Purchase Exposition, St. Louis, Mo., 1904; E. W. Parker, J. A. Holmes, M. R. Campbell, committee in charge. $1906.1,492$ pp., 13 pls.

B 290. Preliminary report on the operations of the fuel-testing plant of the United States Geologica] Survey at St. Louis, Mo., 1905, by J. A. Holmes. 1906.240 pp.

B 305. The analysis of silicate and carbonate rocks, by W. F. Hillebrand. 1906. 200 pp.

Correspondence should be addressed to

The Director,

United States Geological Survey, Washington, D. C.

JANUARY, 190\% . 








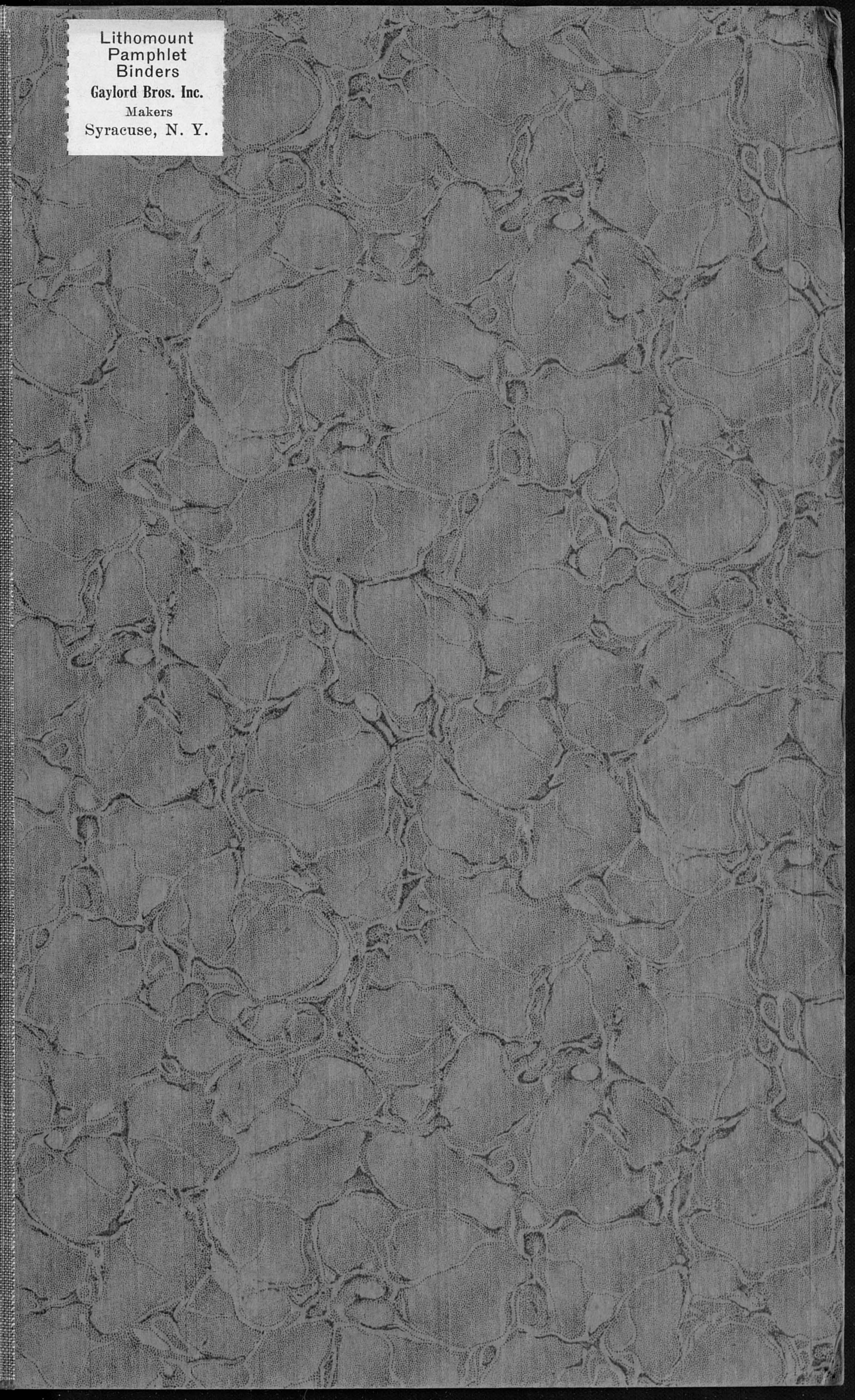




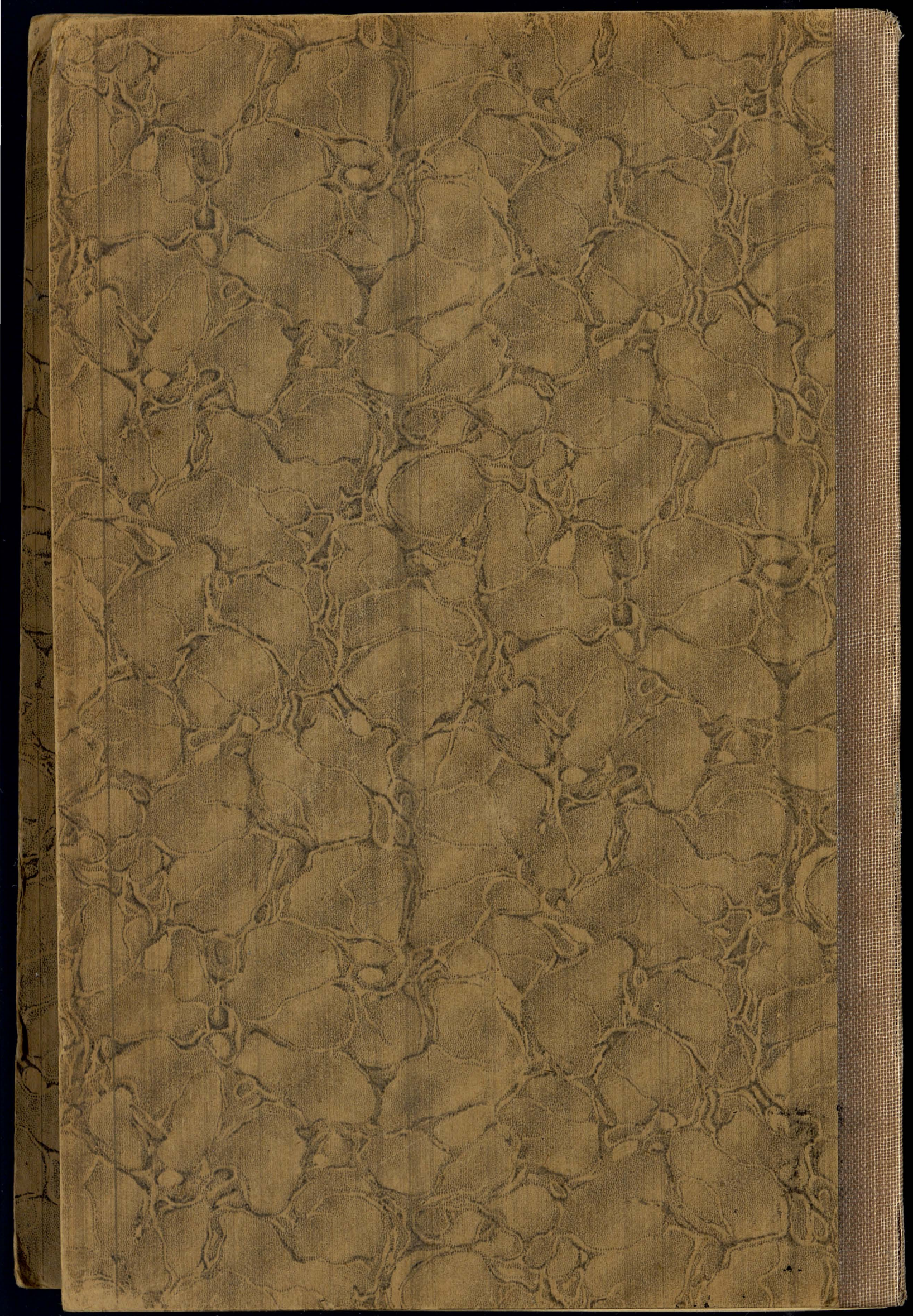

